NASA Reference Publication 1065

\title{
Motion About the Stable Libration Points in the Linearized, Restricted Three-Body Problem
}

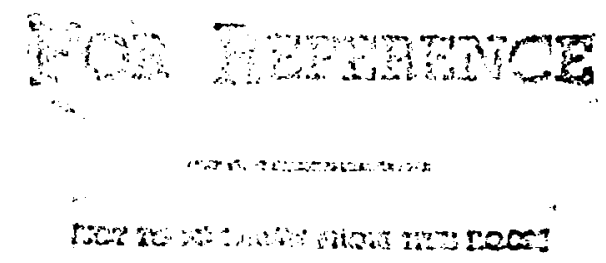

Don Mittleman 

NASA Reference Publication 1065

\section{Motion About the Stable Libration Points in the Linearized, Restricted Three-Body Problem}

Don Mittleman

Oberlin College

Oberlin, Obio

\section{N/SN \\ National Aeronautics \\ and Space Administration}

Scientific and Technical

Information Branch 



\section{CONTENTS}

Section

Page

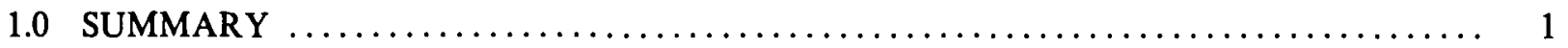

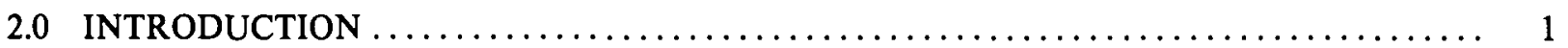

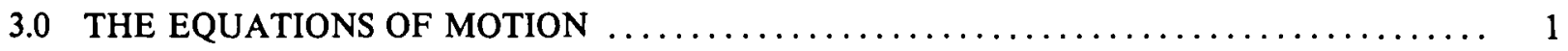

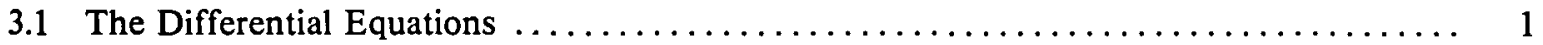

3.2 Solutions of the Differential Equations $\ldots \ldots \ldots \ldots \ldots \ldots \ldots \ldots \ldots \ldots \ldots \ldots \ldots \ldots \ldots$

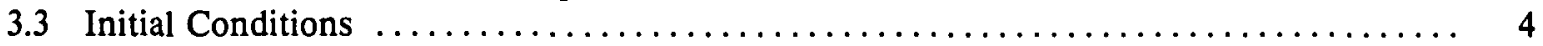

3.4 Some Useful Relationships $\ldots \ldots \ldots \ldots \ldots \ldots \ldots \ldots \ldots \ldots \ldots \ldots \ldots \ldots \ldots \ldots \ldots$

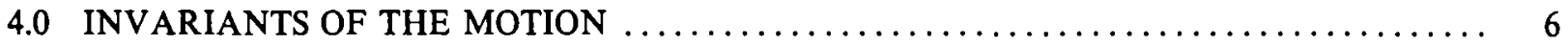

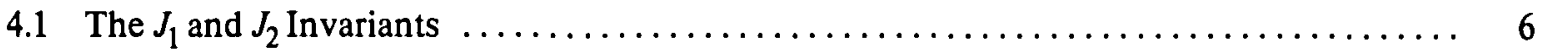

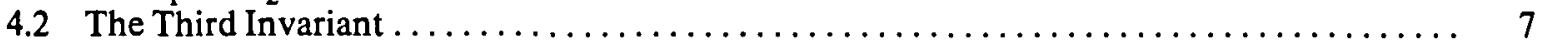

4.3 On the Relation Between $J_{1}, J_{2}$, and $C$, the Jacobi Constant $\ldots \ldots \ldots \ldots \ldots \ldots \ldots \ldots \ldots$

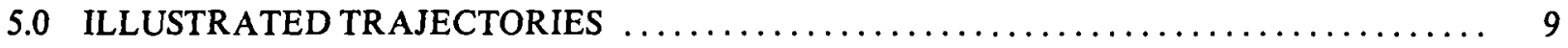

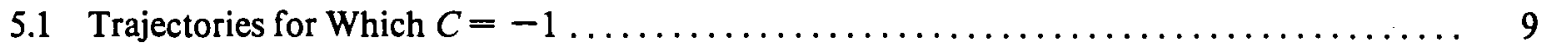

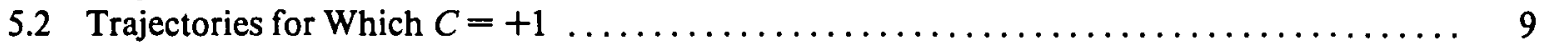

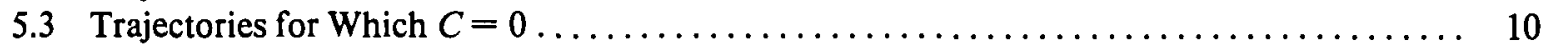

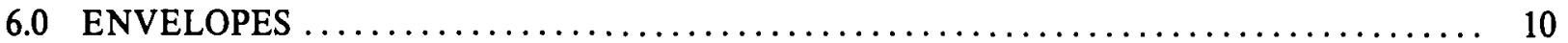

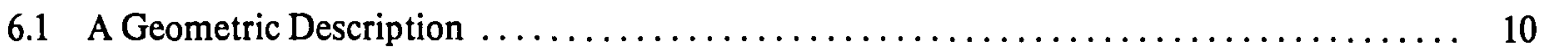

6.2 The Equation of the Envelope $\ldots \ldots \ldots \ldots \ldots \ldots \ldots \ldots \ldots \ldots \ldots \ldots \ldots \ldots \ldots \ldots \ldots \ldots$

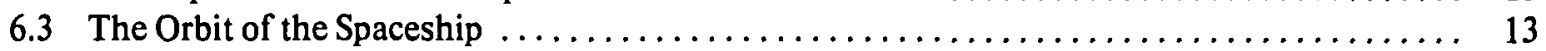

6.4 An Approximation to the Envelope $\ldots \ldots \ldots \ldots \ldots \ldots \ldots \ldots \ldots \ldots \ldots \ldots \ldots \ldots \ldots \ldots$

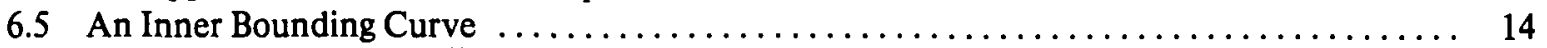

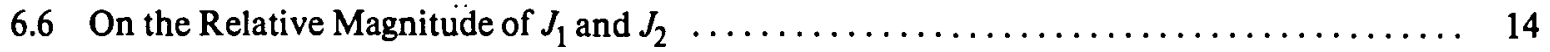

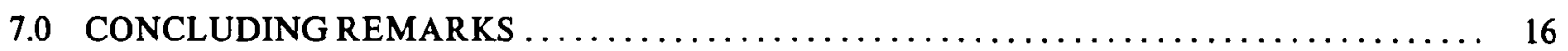

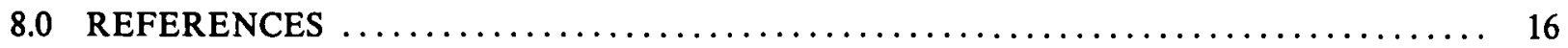

APPENDIX-PARAMETERIZATIONS FOR THE JACOBI ELLIPSE $\ldots \ldots \ldots \ldots \ldots \ldots \ldots$ 
1 Typical trajectories for which $C=-1$

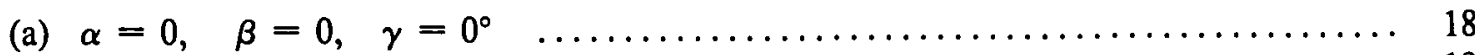

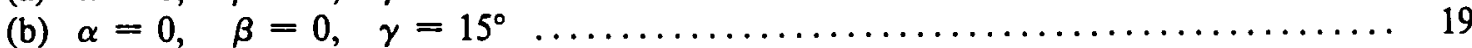

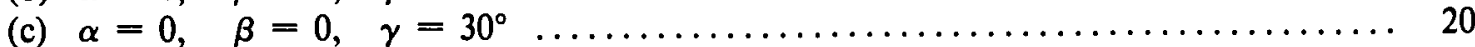

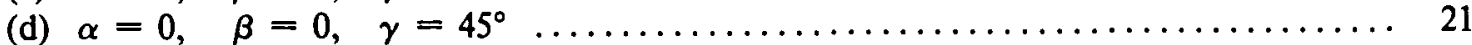

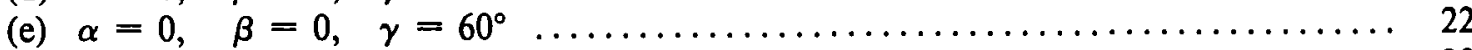

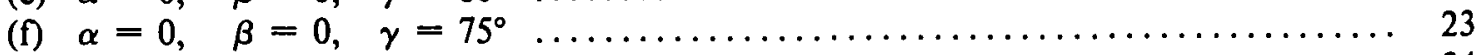

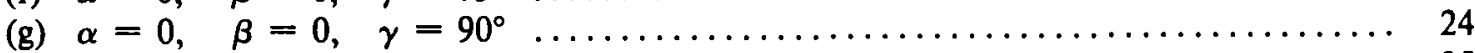

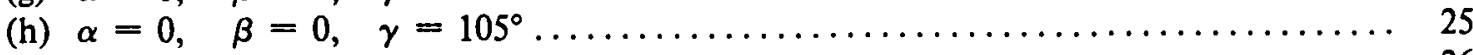

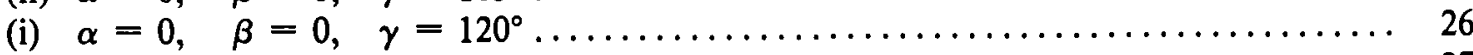

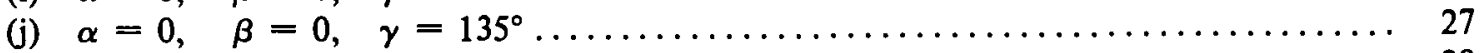

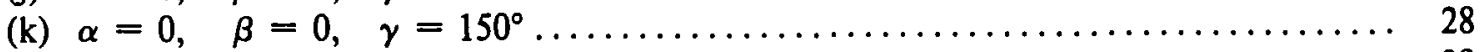

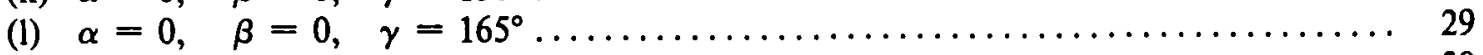

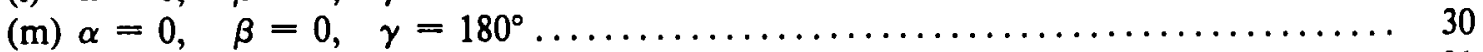

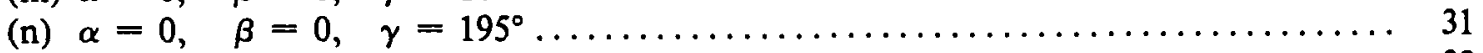

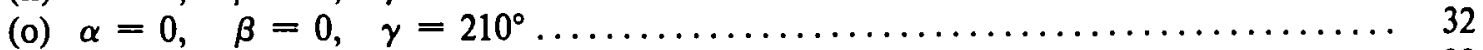

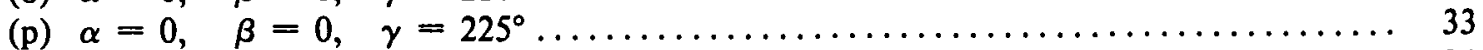

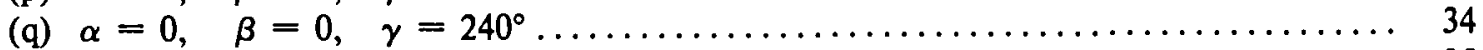

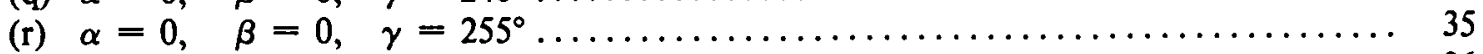

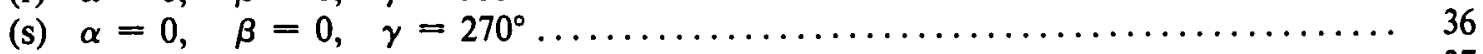

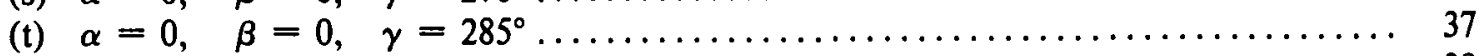

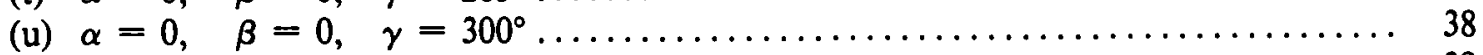

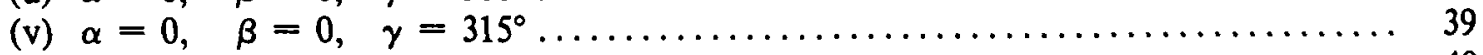

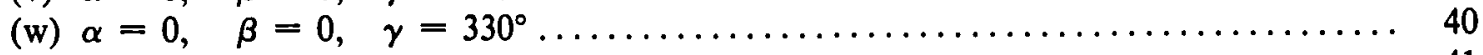

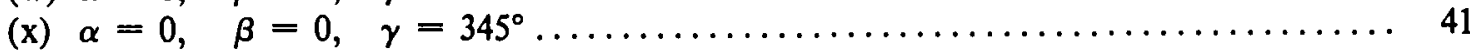

2 Typical trajectories for which $C=+1$

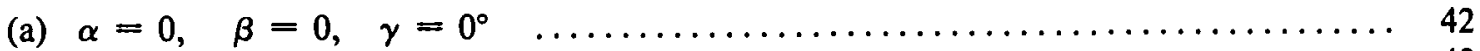

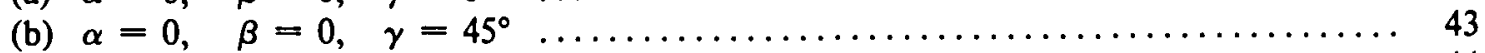

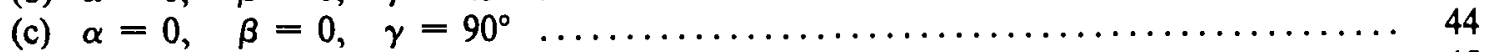

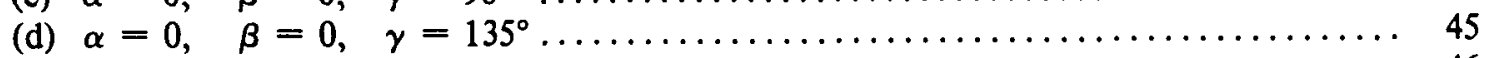

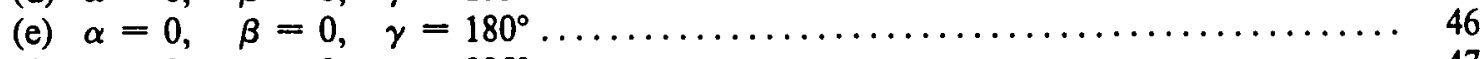

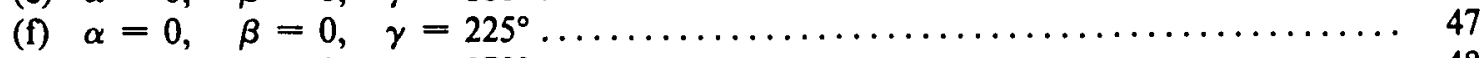

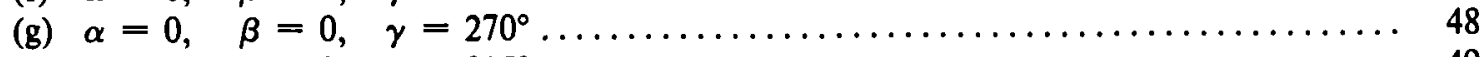

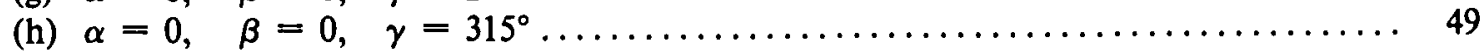

3 Typical trajectories for which $C=0$

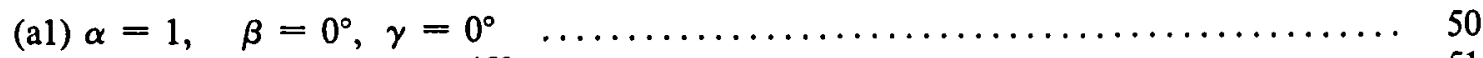

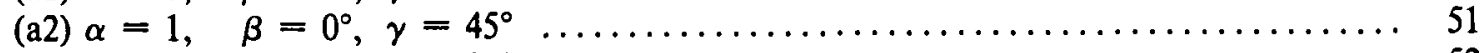

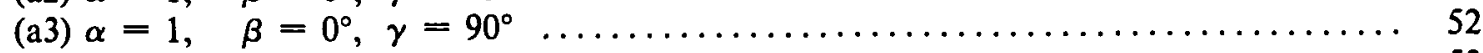

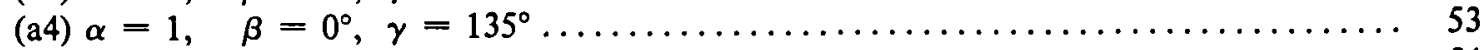

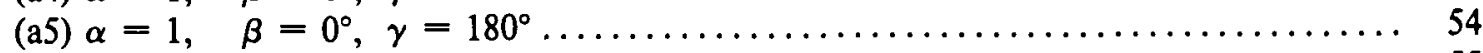

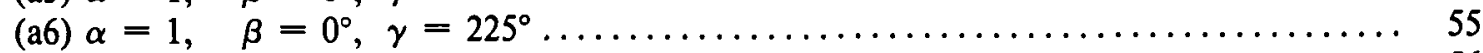

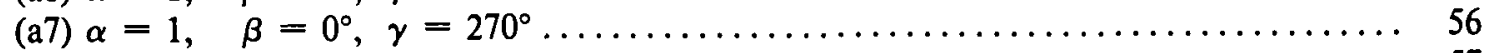

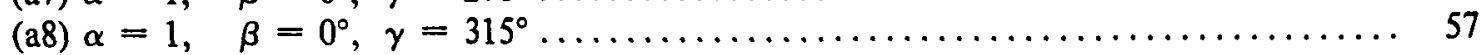


3 Typical trajectories for which $C=0$ (continued)

(b1) $\alpha=1, \quad \beta=45^{\circ}, \quad \gamma=0^{\circ}$

(b2) $\alpha=1, \quad \beta=45^{\circ}, \gamma=45^{\circ}$

(b3) $\alpha=1, \quad \beta=45^{\circ}, \quad \gamma=90^{\circ}$

(b4) $\alpha=1, \quad \beta=45^{\circ}, \quad \gamma=135^{\circ}$

(b5) $\alpha=1, \quad \beta=45^{\circ}, \gamma=180^{\circ}$

(b6) $\quad \alpha=1, \quad \beta=45^{\circ}, \quad \gamma=225^{\circ}$

(b7) $\alpha=1, \quad \beta=45^{\circ}, \quad \gamma=270^{\circ}$

(b8) $\alpha=1, \quad \beta=45^{\circ}, \gamma=315^{\circ}$

(c1) $\alpha=1, \quad \beta=90^{\circ}, \gamma=0^{\circ}$

(c2) $\alpha=1, \quad \beta=90^{\circ}, \gamma=45^{\circ}$

(c3) $\alpha=1, \quad \beta=90^{\circ}, \gamma=90^{\circ}$

(c4) $\alpha=1, \quad \beta=90^{\circ}, \gamma=135^{\circ}$

(c5) $\alpha=1, \quad \beta=90^{\circ}, \quad \gamma=180^{\circ}$

(c6) $\alpha=1, \quad \beta=90^{\circ}, \quad \gamma=225^{\circ}$

(c7) $\alpha=1, \quad \beta=90^{\circ}, \quad \gamma=270^{\circ}$

(c8) $\alpha=1, \quad \beta=90^{\circ}, \quad \gamma=315^{\circ}$

(d1) $\alpha=1, \quad \beta=135^{\circ}, \gamma=0^{\circ}$

(d2) $\alpha=1, \quad \beta=135^{\circ}, \gamma=45^{\circ}$

(d3) $\alpha=1, \quad \beta=135^{\circ}, \gamma=90^{\circ}$

(d4) $\alpha=1, \quad \beta=135^{\circ}, \gamma=135^{\circ}$

(d5) $\alpha=1, \quad \beta=135^{\circ}, \gamma=180^{\circ}$

(d6) $\alpha=1, \quad \beta=135^{\circ}, \gamma=225^{\circ}$

(d7) $\alpha=1, \quad \beta=135^{\circ}, \gamma=270^{\circ}$

(d8) $\alpha=1, \quad \beta=135^{\circ}, \gamma=315^{\circ}$

(e1) $\alpha=1, \beta=180^{\circ}, \gamma=0^{\circ}$

(e2) $\alpha=1, \quad \beta=180^{\circ}, \gamma=45^{\circ}$

(e3) $\alpha=1, \quad \beta=180^{\circ}, \gamma=90^{\circ}$

(e4) $\alpha=1, \quad \beta=180^{\circ}, \gamma=135^{\circ}$

(e5) $\alpha=1, \quad \beta=180^{\circ}, \gamma=180^{\circ}$

(e6) $\alpha=1, \quad \beta=180^{\circ}, \gamma=225^{\circ}$

(e7) $\alpha=1, \quad \beta=180^{\circ}, \gamma=270^{\circ}$

(e8) $\alpha=1, \quad \beta=180^{\circ}, \gamma=315^{\circ}$

(f1) $\alpha=1, \quad \beta=225^{\circ}, \gamma=0^{\circ}$

(f2) $\alpha=1, \quad \beta=225^{\circ}, \gamma=45^{\circ}$

(f3) $\alpha=1, \quad \beta=225^{\circ}, \gamma=90^{\circ}$

(f4) $\alpha=1, \quad \beta=225^{\circ}, \gamma=135^{\circ}$

(f5) $\alpha=1, \quad \beta=225^{\circ}, \gamma=180^{\circ}$

(f6) $\alpha=1, \quad \beta=225^{\circ}, \gamma=225^{\circ}$

(f) $\alpha=1, \quad \beta=225^{\circ}, \gamma=270^{\circ}$

(f8) $\alpha=1, \quad \beta=225^{\circ}, \gamma=315^{\circ}$ 
3 Typical trajectories for which $C=0$ (concluded)

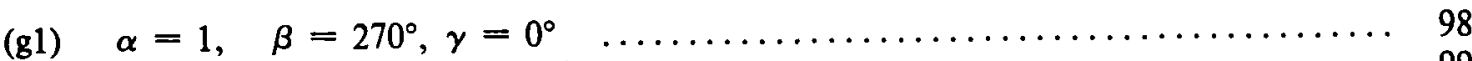

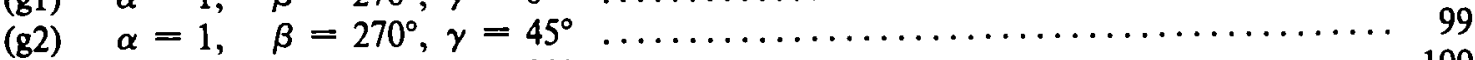

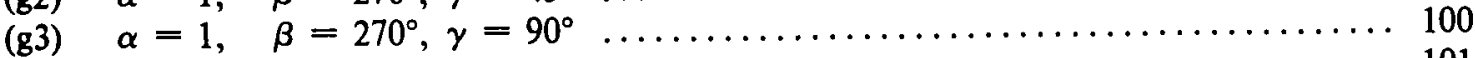

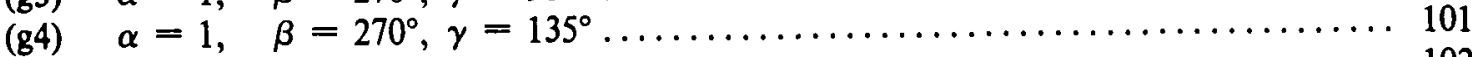

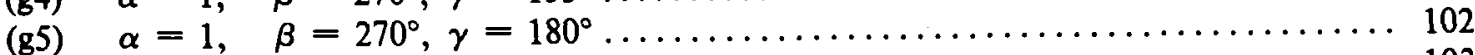

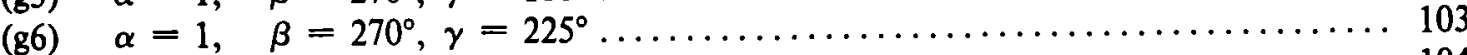

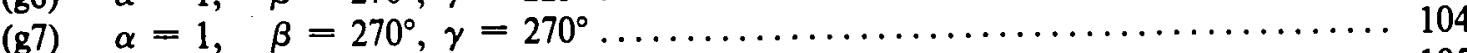

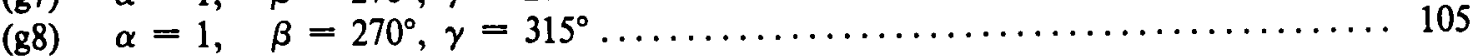

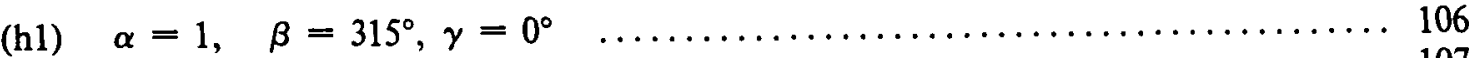

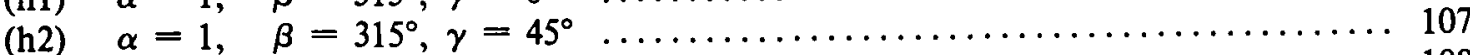

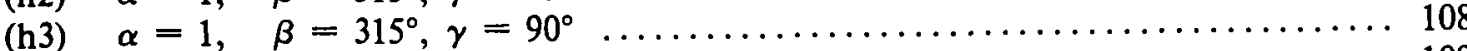

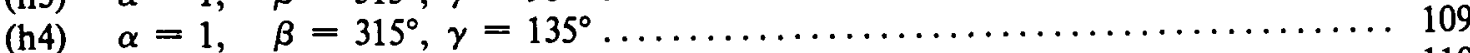

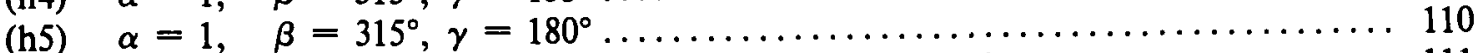

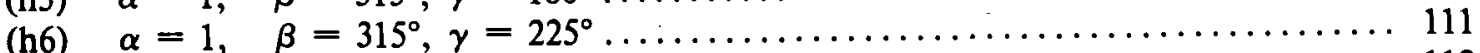

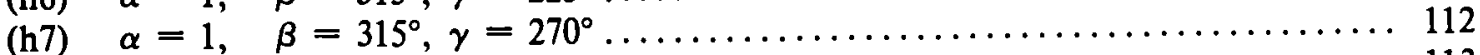

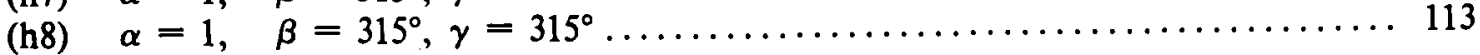

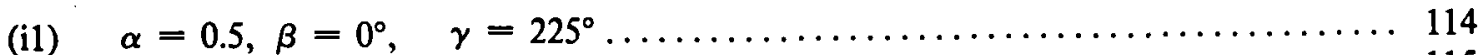

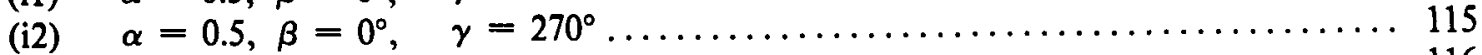

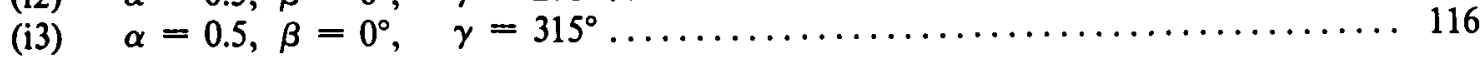

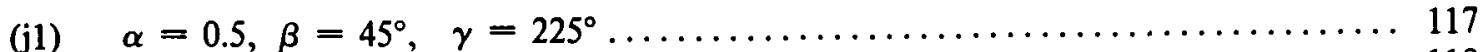

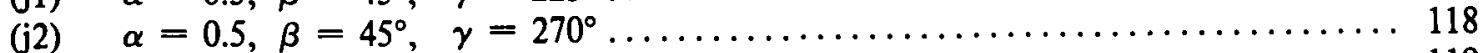

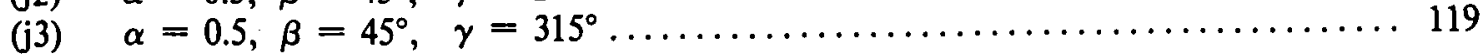

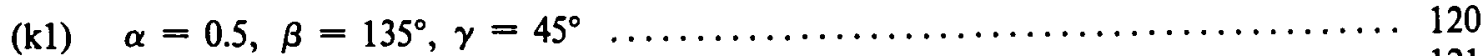

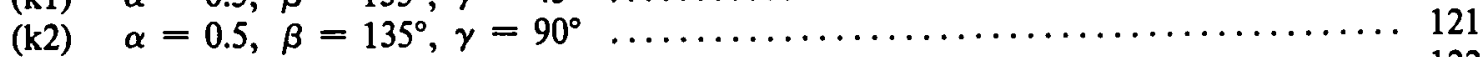

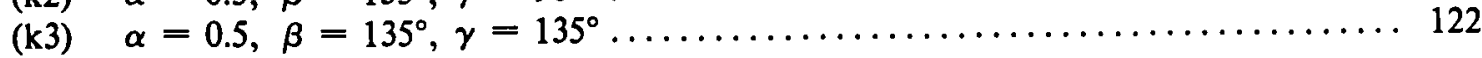

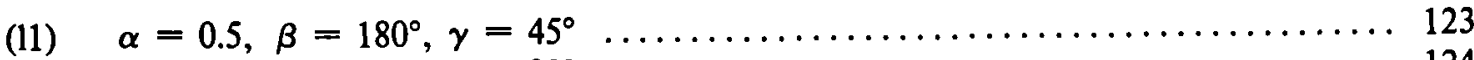

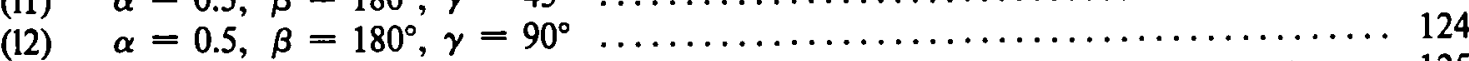

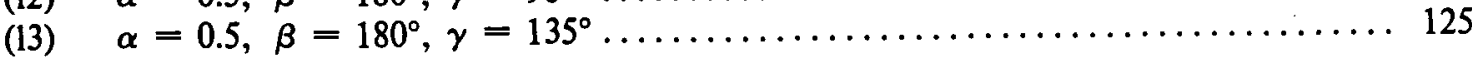

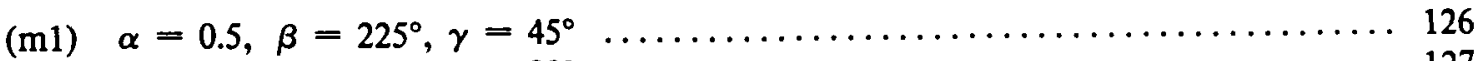

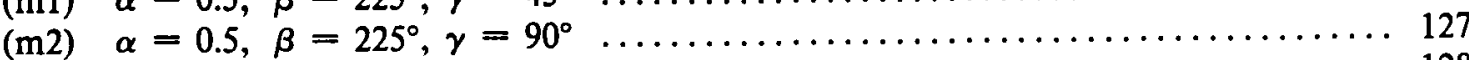

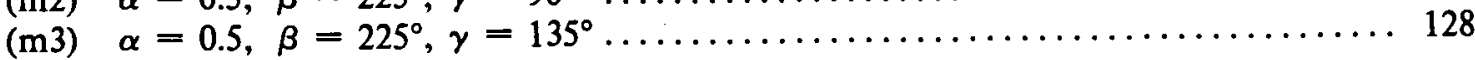

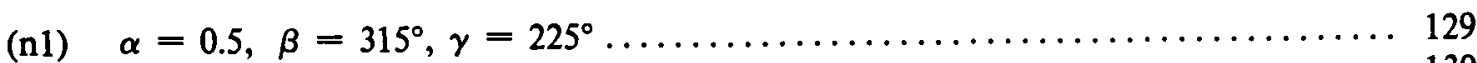

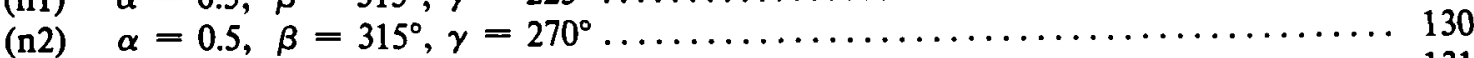

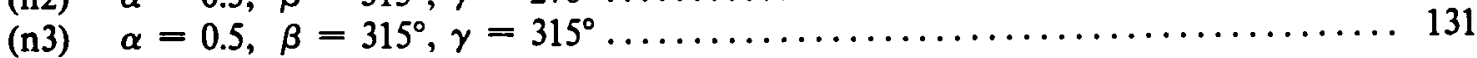


4 Comparison of approximate and exact bounds

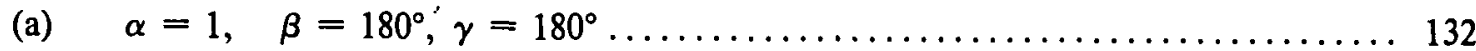

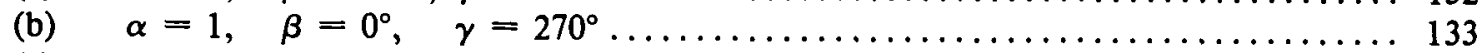

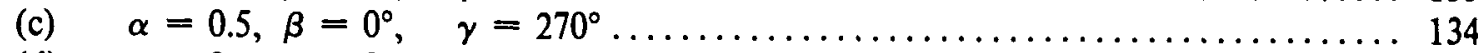

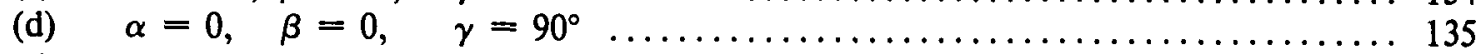

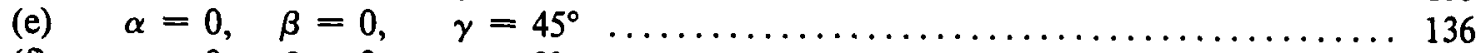

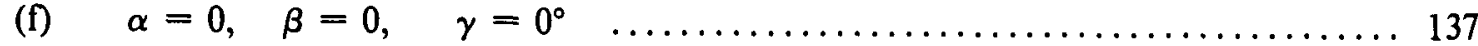

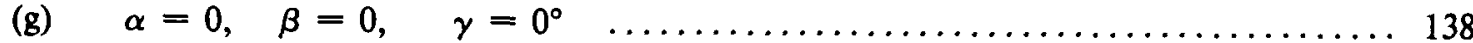

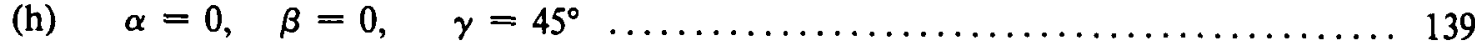

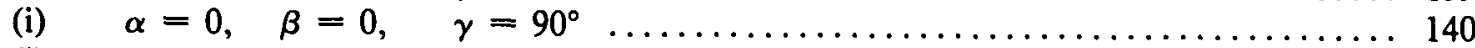

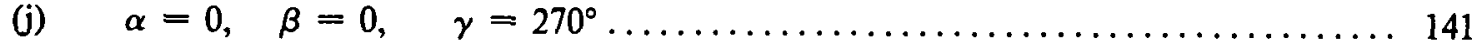

$5 \quad$ Typical inner bounding curve showing excluded region $\left(\alpha=0.5, \beta=0, \gamma=270^{\circ}\right) \ldots 142$

$6 \quad$ Family of smaller ellipses $\left(\alpha=0.5, \beta=0, \gamma=270^{\circ}\right) \ldots \ldots \ldots \ldots \ldots \ldots \ldots \ldots \ldots$

$7 \quad$ An example for which there is no inner excluded region $\left(\alpha=0, \beta=0, \gamma=45^{\circ}\right) \ldots 144$

8 Trajectories for which $J_{1}>J_{2}$

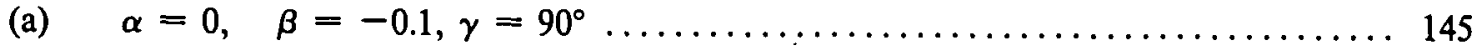

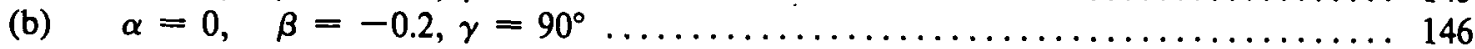

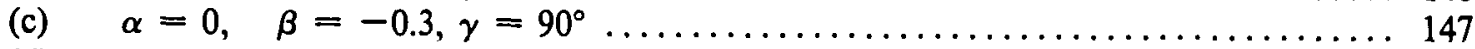

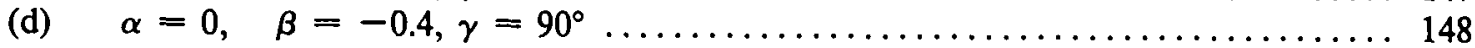

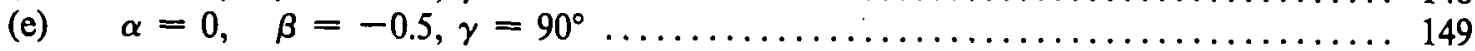

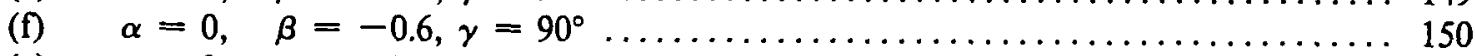

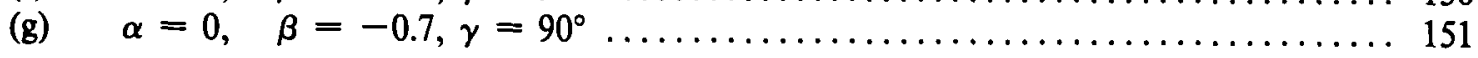





\title{
Motion About the Stable Libration Points in the Linearized, Restricted Three-Body Problem
}

\author{
Don Mittleman*
}

\subsection{SUMMARY}

Starting from the well-known differential equations of motion for a point particle in the neighborhood of a triangle libration point $\left(L_{4}\right.$ or $\left.L_{5}\right)$ in the linearized, restricted problem of three bodies in the plane, and after obtaining the finite equations of motion, three integral invariants of the motion are derived. The Jacobi integral is expressed linearly in terms of two of these. The trajectories for varied initial conditions are drawn; the motion of a point particle, as it moves along its trajectory, can be visualized in elementary geometric terms. Each trajectory has an exterior bounding curve; its equation is found. An approximation to this boundary curve, a well-defined ellipse, had been known; the two curves are compared graphically. For certain initial conditions, there is an interior region from which the trajectory is excluded; the equation of the boundary of this region is found.

\subsection{INTRODUCTION}

The motion of a particle in the neighborhood of a triangular libration point in the linearized, restricted problem of three bodies in the plane is investigated. In section 3, the linearized equations of motion are given; in section 4 , three invariants of the motion are determined; and, in section 5, the trajectories for several initial conditions are discussed. All trajectories are bounded; thus, in section 6 , the equations for the exterior bounding curve are derived and, where appropriate, the equations for the interior boundary curves are given.

\subsection{THE EQUATIONS OF MOTION}

In section 3.1 , the differential equations of motion for a particle are derived. The Jacobi integral and the

- Oberlin College, Oberlin, Ohio.
Jacobi ellipse are obtained. Generic solutions of the differential equations are given in section 3.2 and their dependency on initial conditions is shown in section 3.3. Various combinations of the constants of the system are interrelated; these relationships are given in section 3.4 .

\subsection{The Differential Equations}

The following discussion parallels closely that given in reference 1 . Two bodies of finite mass, shown as the Earth $(E)$ and the Moon $(M)$ in the following sketch, are assumed to rotate about an axis $\zeta_{1}$ through their center of mass in circular orbits lying in a plane $\pi$ that is orthogonal to $\zeta_{1}$. A rotating rectangular coordinate system $\left(\xi_{1}, \eta_{1}\right)$ lies in $\pi$; the Earth and Moon have the fixed coordinates $\left(x_{1}, 0,0\right)$ and $\left(x_{2}, 0,0\right)$, respectively, and their center of mass is at $(0,0,0)$. The distance between $E$ and $M$ is chosen to be equal to 1 ; the mass of $M$ is taken as $\mu \leqslant 0.5$, that of $E$ as $1-\mu$. The choice for the unit of time is such that the gravitational constant $k^{2}=1.1$

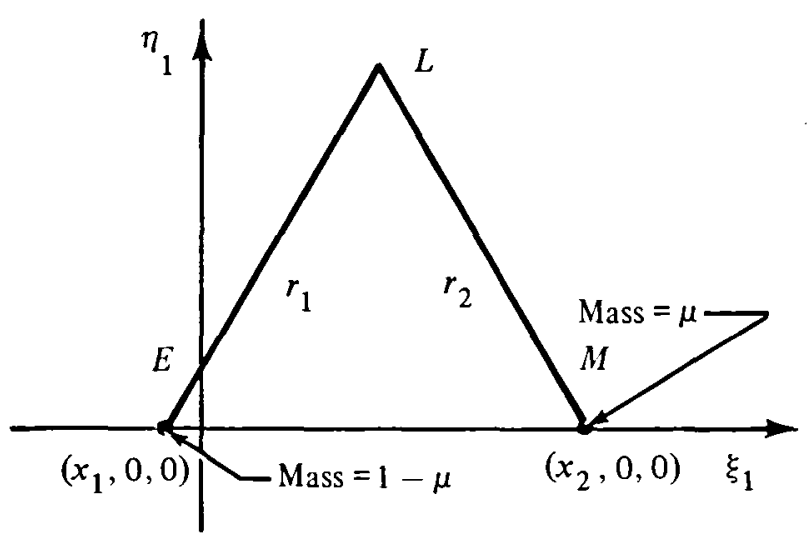

'For the Earth-Moon system, time $t^{*}$ is calculated from this dimensionless time $t$ using the equation $t^{*}=\left(T_{\mathrm{q}} / 2 \pi\right) t$, where $T_{\mathrm{C}}$ is the time for one revolution of the system about the center of mass. For $T_{\mathrm{c}}=27.322$ days, $t^{*}=4.348 t$. 
In the three-dimensional $\left(\xi_{1}, \eta_{1}, \zeta_{1}\right)$ coordinate system, the differential equations of motion for a point particle are

$\frac{d^{2} \xi_{1}}{d t^{2}}-2 \frac{d \eta_{1}}{d t}=\xi_{1}-\frac{(1-\mu)\left(\xi_{1}-x_{1}\right)}{r_{1}{ }^{3}}-\frac{\mu\left(\xi_{1}-x_{2}\right)}{r_{2}{ }^{3}}$

$\frac{d^{2} \eta_{1}}{d t^{2}}+2 \frac{d \xi_{1}}{d t}=\eta_{1}-\frac{(1-\mu) \eta_{1}}{r_{1}{ }^{3}}-\frac{\mu \eta_{1}}{r_{2}{ }^{3}}$

$\frac{d^{2} \zeta_{1}}{d t^{2}}=-\frac{(1-\mu) \zeta_{1}}{r_{1}{ }^{3}}-\frac{\mu \zeta_{1}}{r_{2}{ }^{3}}$

where $r_{1}^{2}=\left(\xi_{1}-x_{1}\right)^{2}+\eta_{1}^{2}+\zeta_{1}^{2}$ and $r_{2}^{2}=\left(\xi_{1}-\right.$ $\left.x_{2}\right)^{2}+\eta_{1}^{2}+\zeta_{1}^{2}$. If the potential function

$$
U\left(\xi_{1}, \eta_{1}, \xi_{1}\right)=\frac{1}{2}\left(\xi_{1}^{2}+\eta_{1}^{2}\right)+\frac{(1-\mu)}{r_{1}}+\frac{\mu}{r_{2}}
$$

is introduced, the equations of motion become

$$
\begin{aligned}
& \frac{d^{2} \xi_{1}}{d t^{2}}-2 \frac{d \eta_{1}}{d t}=\frac{\partial U}{\partial \xi_{1}} \\
& \frac{d^{2} \eta_{1}}{d t^{2}}+2 \frac{d \xi_{1}}{d t}=\frac{\partial U}{\partial \eta_{1}} \\
& \frac{d^{2} \zeta_{1}}{d t^{2}}=\frac{\partial U}{\partial \zeta_{1}}
\end{aligned}
$$

If a $\left(\xi_{2}, \eta_{2}, \zeta_{2}\right)$ coordinate system is defined with the origin at $L$, a triangular libration point, and with the axes parallel to the $\left(\xi_{1}, \eta_{1}, \zeta_{1}\right)$ axes, the linearized equations of motion are

$$
\begin{aligned}
& \frac{d^{2} \xi_{2}}{d t^{2}}-2 \frac{d \eta_{2}}{d t}=\frac{3}{4} \xi_{2}+\frac{3 \sqrt{3}}{4}(1-2 \mu) \eta_{2} \\
& \frac{d^{2} \eta_{2}}{d t^{2}}+2 \frac{d \xi_{2}}{d t}=\frac{3 \sqrt{3}}{4}(1-2 \mu) \xi_{2}+\frac{9}{4} \eta_{2} \\
& \frac{d^{2} \xi_{2}}{d t^{2}}=-\zeta_{2}
\end{aligned}
$$

Equation (1c), being independent of equations (1a) and $(1 b)$, shows that the motion parallel to the $\zeta_{2}$ axis is periodic with period $2 \pi$. No more will be said about this; the remaining discussion concentrates on the solutions of the first two equations.

If equation (1a) is multiplied by $d \xi_{2} / d t$ and equation (1b) by $d \eta_{2} / d t$ and the results added, the following is obtained.

$\frac{d \xi_{2}}{d t} \frac{d^{2} \xi_{2}}{d t^{2}}+\frac{d \eta_{2}}{d t} \frac{d^{2} \eta_{2}}{d t^{2}}=$

$\frac{3}{4} \xi_{2} \frac{d \xi_{2}}{d t}+\frac{3 \sqrt{3}}{4}(1-2 \mu)\left(\eta_{2} \frac{d \xi_{2}}{d t}+\xi_{2} \frac{d \eta_{2}}{d t}\right)+\frac{9}{4} \eta_{2} \frac{d \eta_{2}}{d t}$

This equation can be integrated and yields the Jacobi integral

$$
\begin{aligned}
\frac{1}{2}\left[\left(\frac{d \xi_{2}}{d t}\right)^{2}+\left(\frac{d \eta_{2}}{d t}\right)^{2}\right]= & \frac{3}{4} \frac{\xi_{2}^{2}}{2}+\frac{3 \sqrt{3}}{4}(1-2 \mu) \xi_{2} \eta_{2} \\
& +\frac{9}{4} \frac{\eta_{2}^{2}}{2}+\frac{C}{2}
\end{aligned}
$$


where $C$ is a constant to be determined from the initial conditions. In particular, three different values for $C$ are distinguished: $-1,0$, and +1 . Since equations (1a) and (1b) are linear in $\xi_{2}$ and $\eta_{2}$ and equation (2) is a homogeneous function of $\xi_{2}, \eta_{2}, d \xi_{2} / d t$, and $d \eta_{2} / d t$, any other value of $C$ can be reduced to one of these three by properly scaling the $\left(\xi_{2}, \eta_{2}\right)$ variables.

3.1.1 Rest trajectories.-Those trajectories for which the initial velocity is zero will be called "rest trajectories." For these, $C=-1$ and the initial positions lie on the conic

$$
\frac{3}{8} \xi_{2}^{2}+\frac{3 \sqrt{3}}{4}(1-2 \mu) \xi_{2} \eta_{2}+\frac{9}{8} \eta_{2}^{2}=\frac{1}{2}
$$

If $0<\mu<1$, the discriminant of the quadratic is

$$
D=\left[\frac{3 \sqrt{3}}{4}(1-2 \mu)\right]^{2}-4\left(\frac{27}{64}\right)<0
$$

and the conic is an ellipse which shall be called the Jacobi ellipse.

3.1.2 Simplified form of the equations of motion.To simplify subsequent discussions, an $(x, y)$ coordinate system is introduced. The origin is taken at $L$, which is also the center of the Jacobi ellipse; the $x$ axis coincides in direction with the major axis of the ellipse and the $y$ axis with the minor axis. This is effectively a rotation of axes with respect to the $\left(\xi_{2}, \eta_{2}\right)$ axes through an angle $\theta$, where $\theta=0.5$ arctan $[-\sqrt{3}(1-2 \mu)]$ radians.

In the $(x, y)$ coordinate system, the linearized equations of motion (equations (1a) and (1b)) become

$$
\begin{aligned}
& \ddot{x}-2 \dot{y}=\omega_{1}{ }^{2} x \\
& \ddot{y}+2 \dot{x}=\omega_{2}{ }^{2} y
\end{aligned}
$$

where

$$
\begin{aligned}
& \omega_{1}{ }^{2}=\frac{3}{2}\left(1-\sqrt{1-3 \mu+3 \mu^{2}}\right) \\
& \omega_{2}{ }^{2}=\frac{3}{2}\left(1+\sqrt{1-3 \mu+3 \mu^{2}}\right)
\end{aligned}
$$

and the dots indicate differentiation with respect to time.

In this coordinate system, the Jacobi integral is

$$
\dot{x}^{2}+\dot{y}^{2}=\omega_{1}{ }^{2} x^{2}+\omega_{2}{ }^{2} y^{2}+C
$$

and the Jacobi ellipse is

$$
\omega_{1}^{2} x^{2}+\omega_{2}^{2} y^{2}=1
$$

\subsection{Solutions of the Differential Equations}

The solutions to equations (3a) and (3b) are of the form

$$
\begin{aligned}
& x=K_{1} \cos \lambda t+K_{2} \sin \lambda t \\
& y=L_{1} \cos \lambda t+L_{2} \sin \lambda t
\end{aligned}
$$

Nonzero solutions can be obtained if $\lambda$ satisfies the characteristic equation:

$$
\begin{aligned}
& \lambda^{4}-\lambda^{2}+\frac{27}{4} \mu(1-\mu)=0 \\
& \lambda_{1}{ }^{2}=\frac{1+\sqrt{1-27 \mu(1-\mu)}}{2} \\
& \lambda_{2}{ }^{2}=\frac{1-\sqrt{1-27 \mu(1-\mu)}}{2} \\
& \lambda_{3}=-\lambda_{1} \\
& \lambda_{4}=-\lambda_{2}
\end{aligned}
$$


Only the stable solutions are considered; i.e., those for which the $\lambda$ are real and not equal to zero. Acceptable values of $\mu$ are those for which $1-$ $27 \mu(1-\mu)>0$ or $\mu<0.0385207 \ldots$. For the Earth-Moon system, $\mu=1 / 82.45 \approx 0.0121285 \ldots$ and the linear theory may be used to describe the motion of a satellite near the libration point.

The solutions to equations (3a) and (3b) are among the functions

$$
\begin{aligned}
& x=K_{1} \cos \lambda_{1} t+K_{2} \sin \lambda_{1} t+K_{3} \cos \lambda_{2} t+K_{4} \sin \lambda_{2} t \\
& y=L_{1} \cos \lambda_{1} t+L_{2} \sin \lambda_{1} t+L_{3} \cos \lambda_{2} t+L_{4} \sin \lambda_{2} t
\end{aligned}
$$

where $K_{1}, \ldots, L_{4}$ are arbitrary constants. If these trial solutions are to satisfy equations (3a) and (3b), the coefficients $K_{1}, \ldots, L_{4}$ are related. The solutions to equations (3a) and ( $3 b$ ) can be represented by

$$
\begin{aligned}
x= & \left(K_{1} \cos \lambda_{1} t+K_{2} \sin \lambda_{1} t\right) \\
& +\left(K_{3} \cos \lambda_{2} t+K_{4} \sin \lambda_{2} t\right) \\
y= & M_{1}\left(K_{2} \cos \lambda_{1} t-K_{1} \sin \lambda_{1} t\right) \\
& +M_{2}\left(K_{4} \cos \lambda_{2} t-K_{3} \sin \lambda_{2} t\right)
\end{aligned}
$$

where

$$
M_{1}=\frac{\omega_{1}{ }^{2}+\lambda_{1}{ }^{2}}{2 \lambda_{1}}
$$

and

$$
M_{2}=\frac{\omega_{1}{ }^{2}+\lambda_{2}{ }^{2}}{2 \lambda_{2}}
$$

\subsection{Initial Conditions}

The dependency of $K_{1}, K_{2}, K_{3}$, and $K_{4}$ on the initial conditions follows. If, at time $t=0, x=x_{0}, y=$ $y_{o}, \dot{x}=\dot{x}_{o}$, and $\dot{y}=\dot{y}_{o}$, then

$$
\begin{aligned}
& K_{1}=\frac{\left(\omega_{1}^{2}+\lambda_{2}^{2}\right) x_{o}+2 y_{o}}{\lambda_{2}{ }^{2}-\lambda_{1}{ }^{2}} \\
& K_{2}=\frac{\lambda_{1}}{\omega_{1}{ }^{2}}\left[\frac{2 \lambda_{2}^{2} y_{o}-\left(\omega_{1}^{2}+\lambda_{2}{ }^{2}\right) \dot{x}_{o}}{\lambda_{2}{ }^{2}-\lambda_{1}{ }^{2}}\right] \\
& \left.K_{3}=-\frac{\left(\omega_{1}^{2}+\lambda_{1}{ }^{2}\right) x_{o}+2 \dot{y}_{o}}{\lambda_{2}{ }^{2}-\lambda_{1}{ }^{2}}\right] \\
& K_{4}=\frac{\lambda_{2}}{\omega_{1}^{2}}\left[\frac{\left(\omega_{1}^{2}+\lambda_{1}^{2}\right) \dot{x}_{o}-2 \lambda_{1}{ }^{2} y_{o}}{\lambda_{2}{ }^{2}-\lambda_{1}{ }^{2}}\right]
\end{aligned}
$$

The determination of $K_{i}(i=1,2,3,4)$ is obtained from equations (4a) and (4b) in a straightforward, if tedious, calculation.

\subsection{Some Useful Relationships}

By direct computation, it can be shown that

$$
\begin{aligned}
& \omega_{1}^{2}+\omega_{2}^{2}=3 \\
& \lambda_{1}^{2}+\lambda_{2}^{2}=1 \\
& \omega_{1} \omega_{2}=\lambda_{1} \lambda_{2}
\end{aligned}
$$

$$
\begin{aligned}
& M_{1}=\frac{\omega_{1}^{2}+\lambda_{1}^{2}}{2 \lambda_{1}}=\frac{2 \lambda_{1}}{\omega_{2}{ }^{2}+\lambda_{1}{ }^{2}} \\
& M_{2}=\frac{\omega_{1}^{2}+\lambda_{2}{ }^{2}}{2 \lambda_{2}}=\frac{2 \lambda_{2}}{\omega_{2}{ }^{2}+\lambda_{2}{ }^{2}}
\end{aligned}
$$


and

Since $\lambda_{i}(i=1,2)$ is a root of the characteristic equation (6), which can be written in the form

$$
M_{1} M_{2}=\frac{\omega_{1}}{\omega_{2}}
$$

$$
\frac{\omega_{1}^{2}+\lambda^{2}}{2 \lambda}=\frac{2 \lambda}{\omega_{2}^{2}+\lambda^{2}}
$$

Alternately, if one starts with the equation

$$
\begin{aligned}
& \ddot{x}-2 \dot{y}=\omega_{1}^{2} x \\
& \ddot{y}+2 \dot{x}=\omega_{2}^{2} y
\end{aligned}
$$

and seeks a solution of the form

$$
\begin{aligned}
& x=K_{1} \cos \lambda t+K_{2} \sin \lambda t \\
& y=L_{1} \cos \lambda t+L_{2} \sin \lambda t
\end{aligned}
$$

the characteristic equation for $\lambda$ is

$$
\lambda^{4}+\left(\omega_{1}^{2}+\omega_{2}^{2}-4\right) \lambda^{2}+\omega_{1}^{2} \omega_{2}^{2}=0
$$

From this, it is inferred that

$$
\lambda_{1}^{2}+\lambda_{2}^{2}=-\left(\omega_{1}^{2}+\omega_{2}^{2}-4\right)
$$

or

$$
\lambda_{1}^{2}+\lambda_{2}^{2}+\omega_{1}^{2}+\omega_{2}^{2}=4
$$

and, since $\omega_{1}^{2}+\omega_{2}^{2}=3, \lambda_{1}^{2}+\lambda_{2}^{2}=1$. From $\lambda_{1}^{2} \lambda_{2}^{2}=\omega_{1}^{2} \omega_{2}^{2}$, it is inferred that $\lambda_{1} \lambda_{2}=\omega_{1} \omega_{2}$, disregarding the negative value for the square root.

the alternate forms for $M_{1}$ and $M_{2}$ become apparent. Finally, from

$$
M_{1}=\frac{2 \lambda_{1}}{\omega_{2}^{2}+\lambda_{1}^{2}}
$$

$$
=\frac{2\left(\frac{\omega_{1} \omega_{2}}{\lambda_{2}}\right)}{\omega_{2}^{2}+\lambda_{1}{ }^{2}}
$$$$
=\frac{2 \omega_{1} \omega_{2}}{\lambda_{2} \omega_{2}{ }^{2}\left[1+\left(\frac{\lambda_{1}}{\omega_{2}}\right)^{2}\right]}
$$

$$
=\frac{2 \omega_{1}}{\lambda_{2} \omega_{2}\left[1+\left(\frac{\omega_{1}}{\lambda_{2}}\right)^{2}\right]}
$$

$$
\begin{aligned}
& =\frac{2 \omega_{1} \lambda_{2}}{\omega_{2}\left(\lambda_{2}^{2}+\omega_{1}^{2}\right)} \\
& =\frac{\omega_{1}}{\omega_{2}}\left(\frac{2 \lambda_{2}}{\omega_{1}^{2}+\lambda_{2}^{2}}\right)
\end{aligned}
$$

$$
=\frac{\omega_{1}}{\omega_{2}} \frac{1}{M_{2}}
$$

$$
M_{1} M_{2}=\frac{\omega_{1}}{\omega_{2}}
$$




\subsection{INVARIANTS OF THE MOTION}

In section 4.1, two invariants of the motion, $J_{1}$ and $J_{2}$, are derived. A third invariant relationship is given in section 4.2 , where it is also shown that these three invariants can be used to distinguish between different trajectories or to identify a "second" trajectory as a continuation of an earlier identified one. A linear relation exists between $J_{1}{ }^{2}, J_{2}{ }^{2}$ and the Jacobi constant; this is derived in section 4.3.

\subsection{The $J_{1}$ and $J_{2}$ Invariants}

Suppose a particle $P$ is observed at $t=t_{0}$ at position $\left(x_{o}, y_{0}\right)$ with velocity $\left(\dot{x}_{o}, \dot{y}_{o}\right)$. Its trajectory can be determined. At some later time $t_{1}$, the particle $P$ will be at the point $\left[x\left(t_{1},\right) y\left(t_{1}\right)\right]=\left(x_{1}, y_{1}\right)$ with velocity $\left[\dot{x}\left(t_{1}\right), \dot{y}\left(t_{1}\right)\right]=\left(\dot{x}_{1}, \dot{y}_{1}\right)$. However, between $t=t_{0}$ and $t=t_{1}$, you lose track of the particle. Is it possible to decide if the particle $P$ observed at time $t=t_{0}$ and the particle observed at $t=t_{1}$ are the same? The following discussion partly answers this question.

Having observed the particle initially at $\left(x_{0}, y_{0}\right)$ with "initial" velocity $\left(\dot{x}_{o}, \dot{y}_{o}\right)$, a unique trajectory and, in particular, the numbers $K_{1}, K_{2}, K_{3}$, and $K_{4}$ are determined. At the later time $t_{1}$, a particle is observed at the point $\left(x_{1}, y_{1}\right)$ with velocity $\left(\dot{x}_{1}, \dot{y}_{1}\right)$. For these "initial" conditions, a unique trajectory and, in particular, four numbers $K_{1}^{*}, K_{2}^{*}, K_{3}^{*}$, and $K_{4}^{*}$ are determined. The question may then be framed: Is this second trajectory merely a continuation of the first or is a different particle being observed?

Using $t$ to represent time for the "first" particle (so that at $t_{0}, t=0$ ), its equations of motion are

$$
\begin{aligned}
x(t)= & K_{1} \cos \lambda_{1} t+K_{2} \sin \lambda_{1} t \\
& +K_{3} \cos \lambda_{2} t+K_{4} \sin \lambda_{2} t \\
y(t)= & M_{1}\left(K_{2} \cos \lambda_{1} t-K_{1} \sin \lambda_{1} t\right) \\
& +M_{2}\left(K_{4} \cos \lambda_{2} t-K_{3} \sin \lambda_{2} t\right)
\end{aligned}
$$

Using $\tau$ to represent time for the "second" particle (so that at $t_{1}, \tau=0$ ), its equations of motion are

$$
\begin{aligned}
x(\tau)= & K_{1}^{*} \cos \lambda_{1} \tau+K_{2}^{*} \sin \lambda_{1} \tau \\
& +K_{3}^{*} \cos \lambda_{2} \tau+K_{4}^{*} \sin \lambda_{2} \tau \\
y(\tau)= & M_{1}\left(K_{2}^{*} \cos \lambda_{1} \tau-K_{1}^{*} \sin \lambda_{1} \tau\right) \\
& +M_{2}\left(K_{4}^{*} \cos \lambda_{2} \tau-K_{3}^{*} \sin \lambda_{2} \tau\right)
\end{aligned}
$$

The relation between $t$ and $\tau$ is $t=\tau+t_{1}$.

Equations (7a) and (7b) for the original trajectory $[x(t), y(t)]$ when viewed in the time frame $\tau$ become

$$
\begin{aligned}
x\left(\tau+t_{1}\right)= & K_{1} \cos \lambda_{1}\left(\tau+t_{1}\right)+K_{2} \sin \lambda_{1}\left(\tau+t_{1}\right) \\
& +K_{3} \cos \lambda_{2}\left(\tau+t_{1}\right)+K_{4} \sin \lambda_{2}\left(\tau+t_{1}\right)
\end{aligned}
$$

$$
\begin{aligned}
y\left(\tau+t_{1}\right)= & M_{1}\left[K_{2} \cos \lambda_{1}\left(\tau+t_{1}\right)-K_{1} \sin \lambda_{1}\left(\tau+t_{1}\right)\right] \\
& +M_{2}\left[K_{4} \cos \lambda_{2}\left(\tau+t_{1}\right)-K_{3} \sin \lambda_{2}\left(\tau+t_{1}\right)\right]
\end{aligned}
$$

Using the addition formulas for $\sin \lambda\left(\tau+t_{1}\right)$ and $\cos$ $\lambda\left(\tau+t_{1}\right)$ in equations (9a) and (9b) and equating the coefficients of $\cos \lambda_{1} \tau, \sin \lambda_{1} \tau, \cos \lambda_{2} \tau$, and $\sin \lambda_{2} \tau$ to the corresponding quantities in equations ( $8 \mathrm{a}$ ) and (8b), ${ }^{2}$ it is found that

$$
\begin{aligned}
& K_{1}^{*}=K_{1} \cos \lambda_{1} t_{1}+K_{2} \sin \lambda_{1} t_{1} \\
& K_{2}^{*}=K_{2} \cos \lambda_{1} t_{1}-K_{1} \sin \lambda_{1} t_{1} \\
& K_{3}^{*}=K_{3} \cos \lambda_{2} t_{1}+K_{4} \sin \lambda_{2} t_{1} \\
& K_{4}^{*}=K_{4} \cos \lambda_{2} t_{1}-K_{3} \sin \lambda_{2} t_{1}
\end{aligned}
$$

\footnotetext{
${ }^{2}$ A sufficient condition to permit the equating of the corresponding coefficients is that $\lambda_{1}$ and $\lambda_{2}$ be incommensurate. This is assumed here and throughout the remainder of this paper.
} 
However, equation (10) implies that

$$
K_{1}^{* 2}+K_{2}^{* 2}=K_{1}^{2}+K_{2}^{2}
$$

and

$$
K_{3}^{* 2}+K_{4}^{* 2}=K_{3}^{2}+K_{4}^{2}
$$

Therefore, $K_{1}{ }^{2}+K_{2}{ }^{2}$ and $K_{3}^{2}+K_{4}{ }^{2}$ are invariants of the trajectory. The notations $J_{1}^{2}=K_{1}^{2}+K_{2}^{2}$ and $J_{2}^{2}$ $=K_{3}^{2}+K_{4}^{2}$ shall be used.

\subsection{The Third Invariant}

It has been shown that the quantities $J_{1}$ and $J_{2}$ are invariants of the trajectory. The question of additional invariants is pursued.

From equations (10a) to (10d), it is found that

$$
\begin{aligned}
& \cos \lambda_{1} t_{1}=\frac{K_{1} K_{1}^{*}+K_{2} K_{2}^{*}}{J_{1}{ }^{2}} \\
& \sin \lambda_{1} t_{1}=\frac{K_{2} K_{1}^{*}-K_{1} K_{2}^{*}}{J_{1}{ }^{2}} \\
& \cos \lambda_{2} t_{1}=\frac{K_{3} K_{3}^{*}+K_{4} K_{4}^{*}}{J_{2}{ }^{2}} \\
& \sin \lambda_{2} t_{1}=\frac{K_{4} K_{3}^{*}-K_{3} K_{4}^{*}}{J_{2}{ }^{2}}
\end{aligned}
$$

(Note that, on the right sides of these equations, only $K_{1}^{*}, K_{2}^{*}, K_{3}^{*}$, and $K_{4}^{*}$ depend on $t_{1}$; the other symbols do not.)

Four angles $\phi_{1}, \phi_{1}^{*}, \phi_{2}$, and $\phi_{2}^{*}$ are defined by the following relations:

$$
\begin{array}{ll}
\cos \phi_{1}=\frac{K_{1}}{J_{1}} & \sin \phi_{1}=\frac{K_{2}}{J_{1}} \\
\cos \phi_{1}^{*}=\frac{K_{1}^{*}}{J_{1}^{*}} & \sin \phi_{1}^{*}=\frac{K_{2}^{*}}{J_{1}^{*}} \\
\cos \phi_{2}=\frac{K_{3}}{J_{2}} & \sin \phi_{2}=\frac{K_{4}}{J_{2}} \\
\cos \phi_{2}^{*}=\frac{K_{3}^{*}}{J_{2}^{*}} & \sin \phi_{2}^{*}=\frac{K_{4}^{*}}{J_{2}^{*}}
\end{array}
$$

(Remember that $J_{1}^{*}=J_{1}$ and $J_{2}^{*}=J_{2}$. Also, only $\phi_{1}^{*}$ and $\phi_{2}^{*}$ are functions of $t_{1}$.) Using this notation,

$$
\begin{aligned}
\cos \left(\lambda_{1} t_{1}\right) & =\cos \phi_{1} \cos \phi_{1}^{*}+\sin \phi_{1} \sin \phi_{1}^{*} \\
& =\cos \left(\phi_{1}-\phi_{1}^{*}\right) \\
\sin \left(\lambda_{1} t_{1}\right) & =\sin \phi_{1} \cos \phi_{1}^{*}-\cos \phi_{1} \sin \phi_{1}^{*} \\
& =\sin \left(\phi_{1}-\phi_{1}^{*}\right)
\end{aligned}
$$

Therefore, there exists an integer $n$ such that

$$
\lambda_{1} t_{1}=\phi_{1}-\phi_{1}^{*}+2 n \pi
$$

It is claimed that $n$ is zero. Consider

$$
2 \pi n=\lambda_{1} t_{1}-\phi_{1}+\phi_{1}^{*}
$$

The symbol $\phi_{1}$, which depends on the "initial" conditions at $t_{0}$, is independent of $t_{1}$ and for our purpose is constant. The symbol $\phi_{1}^{*}$ depends on the "initial" 
conditions at $t_{1} ; \phi_{1}^{*}$ is a continuous function of $t_{1}$. Furthermore, if $t_{1}=0, \phi_{1}^{*}=\phi_{1}$. Therefore, the right side of equation (12b) is zero for $t_{1}=0$. Thus, $n=0$ when $t_{1}=0$. But $n$ is an integer and the right side of equation (12b) is a continuous function of $t_{1}$. This implies that $n \equiv 0$ and equation (12a) becomes

$$
\lambda_{1} t_{1}=\phi_{1}-\phi_{1}^{*}
$$

A similar argument shows that

$$
\lambda_{2} t_{1}=\phi_{2}-\phi_{2}^{*}
$$

Eliminating $t_{1}$

$$
\lambda_{2} \phi_{1}-\lambda_{1} \phi_{2}=\lambda_{2} \phi_{1}^{*}-\lambda_{1} \phi_{2}^{*}
$$

The symbol used shall be $J_{3}=\lambda_{2} \phi_{1}-\lambda_{1} \phi_{2}$.

Equations (11) and (13) show that if the same trajectory is observed at two different times, the quantities $J_{1}, J_{2}$, and $J_{3}$ are invariants. Conversely, if two trajectories are given for which the $J_{1}, J_{2}$, and $J_{3}$ of the first are equal, respectively, to the $J_{1}^{*}, J_{2}^{*}$, and $J_{3}^{*}$ of the second, then the two trajectories are not distinct; one is a continuation at some later time of the other. Furthermore, this later time can be determined from either equation (12c) or equation (12d).

\subsection{On the Relation Between $J_{1}, J_{2}$, and $C$, the Jacobi Constant}

The Jacobi integral, equation (3c), is valid for all values of the time $t$ and in particular for $t=0$. The following relationships between $x_{o}, y_{o}, \dot{x}_{o}$, and $\dot{y}_{o}$ and $K_{1}, K_{2}, K_{3}$, and $K_{4}$ can be found from equations (4a) and (4b) and their derivatives with respect to time.

$$
\begin{aligned}
& x_{o}=K_{1}+K_{3} \\
& \dot{x}_{o}=\lambda_{1} K_{2}+\lambda_{2} K_{4}
\end{aligned}
$$

$$
\begin{aligned}
& y_{o}=M_{1} K_{2}+M_{2} K_{4} \\
& \dot{y}_{o}=-\lambda_{1} M_{1} K_{1}-\lambda_{2} M_{2} K_{3}
\end{aligned}
$$

When these values are substituted into equation (3c), that equation becomes

$$
\begin{aligned}
& \left(\lambda_{1}{ }^{2}-\omega_{2}{ }^{2} M_{1}{ }^{2}\right) K_{2}{ }^{2}+\left(\lambda_{1}{ }^{2} M_{1}{ }^{2}-\omega_{1}{ }^{2}\right) K_{1}{ }^{2} \\
& +\left(\lambda_{2}{ }^{2}-\omega_{2}{ }^{2} M_{2}{ }^{2}\right) K_{4}{ }^{2}+\left(\lambda_{2}{ }^{2} M_{2}{ }^{2}-\omega_{1}{ }^{2}\right) K_{3}{ }^{2} \\
& +2\left(\lambda_{1} \lambda_{2}-\omega_{2}{ }^{2} M_{1} M_{2}\right) K_{2} K_{4} \\
& +2\left(\lambda_{1} \lambda_{2} M_{1} M_{2}-\omega_{1}{ }^{2}\right) K_{1} K_{3} \\
& =-C
\end{aligned}
$$

However, since $M_{1} M_{2}=\omega_{1} / \omega_{2}$ and $\lambda_{1} \lambda_{2}=\omega_{1} \omega_{2}$, the coefficients of $K_{2} K_{4}$ and $K_{1} K_{3}$ are zero.

The coefficients of $K_{1}{ }^{2}$ and $K_{2}{ }^{2}$ are shown to be equal by the following. From section 3.4 ,

$$
M_{1}=\frac{\omega_{1}^{2}+\lambda_{1}{ }^{2}}{2 \lambda_{1}}=\frac{2 \lambda_{1}}{\omega_{2}^{2}+\lambda_{1}{ }^{2}}
$$

so that

$$
\begin{aligned}
& M_{1}{ }^{2}=\frac{\omega_{1}{ }^{2}+\lambda_{1}{ }^{2}}{\omega_{2}{ }^{2}+\lambda_{1}{ }^{2}} \\
& \omega_{2}{ }^{2} M_{1}{ }^{2}+\lambda_{1}{ }^{2} M_{1}{ }^{2}=\omega_{1}{ }^{2}+\lambda_{1}{ }^{2} \\
& \lambda_{1}{ }^{2} M_{1}{ }^{2}-\omega_{1}{ }^{2}=\lambda_{1}{ }^{2}-\omega_{2}{ }^{2} M_{1}{ }^{2}
\end{aligned}
$$

A similar calculation shows that the coefficients of 
$K_{3}{ }^{2}$ and $K_{4}{ }^{2}$ are equal. Thus, equation (15) can be written as

$$
\left(\omega_{2}{ }^{2} M_{1}{ }^{2}-\lambda_{1}{ }^{2}\right) J_{1}{ }^{2}+\left(\omega_{2}{ }^{2} M_{2}{ }^{2}-\lambda_{2}{ }^{2}\right) J_{2}{ }^{2}=C
$$

\subsection{ILLUSTRATED TRAJECTORIES}

Trajectories associated with different initial conditions are explored, in a systematic way, in this section. At a first level of classification, trajectories were differentiated on the basis of the value of the Jacobi constant $C$. In sections 5.1, 5.2, and 5.3, trajectories for which $C=-1, C=+1$, and $C=0$, respectively, are examined.

\subsection{Trajectories for Which $C=-1$}

Included among the trajectories for which $C=$ -1 are the rest trajectories, those for which the starting position is on the Jacobi ellipse.

$$
\omega_{1}^{2} x_{o}^{2}+\omega_{2}^{2} y_{o}^{2}=1
$$

These constitute a one-parameter family of solutions that can be parameterized by $x_{o}=\left(1 / \omega_{1}\right) \cos \gamma$ and $y_{o}=\left(1 / \omega_{2}\right) \sin \gamma$, where $\gamma$ is the parameter. The values of $K_{i}$ are

$$
\begin{aligned}
& K_{1}=\frac{\left(\omega_{1}^{2}+\lambda_{2}{ }^{2}\right) \cos \gamma}{\omega_{1}\left(\lambda_{2}{ }^{2}-\lambda_{1}{ }^{2}\right)} \\
& K_{2}=\frac{2 \lambda_{2} \sin \gamma}{\omega_{1}\left(\lambda_{2}{ }^{2}-\lambda_{1}{ }^{2}\right)} \\
& K_{3}=-\frac{\left(\omega_{1}{ }^{2}+\lambda_{1}{ }^{2}\right) \cos \gamma}{\omega_{1}\left(\lambda_{2}{ }^{2}-\lambda_{1}{ }^{2}\right)} \\
& K_{4}=-\frac{2 \lambda_{1} \sin \gamma}{\omega_{1}\left(\lambda_{2}{ }^{2}-\lambda_{1}{ }^{2}\right)}
\end{aligned}
$$

Typical trajectories are drawn for $\gamma=0^{\circ}, 15^{\circ}$, $30^{\circ}, \ldots, 345^{\circ}$ in figure 1 . The value of $\mu=1 / 82.45$ was chosen, from which $\lambda_{1}, \lambda_{2}, \omega_{1}, \omega_{2}, M_{1}$, and $M_{2}$ were calculated.

For the specific initial conditions, $K_{1}, K_{2}, K_{3}, K_{4}$, and $J_{1}, J_{2}$ were also calculated. Each graph is scaled so that each mark represents 5 units. The innermost curve (in black) is the Jacobi ellipse; its semi-major axis equals $1 / \omega_{1}$ and its semi-minor axis equals $1 / \omega_{2}$. The trajectory is drawn in red for $0 \leqslant t \leqslant 50 .{ }^{3}$ The blue curve will be discussed in section 6 .

\subsection{Trajectories for Which $C=+1$}

Among the trajectories for which $C=+1$ are those which start, with velocity different from zero, at the libration point $x=0, y=0$. Under these conditions, the Jacobi integral becomes

$$
\dot{x}_{o}^{2}+\dot{y}_{o}^{2}=1
$$

These trajectories constitute a one-parameter family of solutions that can be parameterized by $\dot{x}_{0}=\cos \gamma$ and $\dot{y}_{o}=\sin \gamma$, where $\gamma$ is the parameter. The values of $K_{i}$ are

$$
\begin{aligned}
& K_{1}=\frac{2 \sin \gamma}{\left(\lambda_{2}{ }^{2}-\lambda_{1}{ }^{2}\right)} \\
& K_{2}=-\frac{\lambda_{1}\left(\omega_{1}^{2}+\lambda_{2}^{2}\right) \cos \gamma}{\omega_{1}^{2}\left(\lambda_{2}{ }^{2}-\lambda_{1}{ }^{2}\right)} \\
& K_{3}=-\frac{2 \sin \gamma}{\left(\lambda_{2}{ }^{2}-\lambda_{1}{ }^{2}\right)} \\
& K_{4}=\frac{\lambda_{2}\left(\omega_{1}{ }^{2}+\lambda_{1}{ }^{2}\right) \cos \gamma}{\omega_{1}{ }^{2}\left(\lambda_{2}{ }^{2}-\lambda_{1}{ }^{2}\right)}
\end{aligned}
$$

Typical trajectories are drawn for $\gamma=0^{\circ}, 45^{\circ}, 90^{\circ}$, $135^{\circ}, 180^{\circ}, 225^{\circ}, 270^{\circ}$, and $315^{\circ}$ in figure 2.

${ }^{3}$ This corresponds to approximately 217.422 days. 


\subsection{Trajectorles for Which $C=0$}

The third possible value for $C$ is zero. Under these conditions, the Jacobi integral becomes

$$
\dot{x}_{o}{ }^{2}+\dot{y}_{o}{ }^{2}=\omega_{1}{ }^{2} x_{o}{ }^{2}+\omega_{2}{ }^{2} y_{o}{ }^{2}
$$

The trajectories to be used as models will be those that start on the ellipse

$$
\omega_{1}^{2} x_{o}^{2}+\omega_{2}^{2} y_{o}^{2}=\alpha^{2}
$$

with the proper initial velocity dictated by the equation

$$
\dot{x}_{o}^{2}+\dot{y}_{o}^{2}=\alpha^{2}
$$

These conditions may be parameterized by $x_{o}=$ $\left(\alpha / \omega_{1}\right) \cos \beta, y_{o}=\left(\alpha / \omega_{2}\right) \sin \beta, \dot{x}_{o}=\alpha \cos \gamma$, and $\dot{y}_{o}$ $=\alpha \sin \gamma$. The values of $K_{i}$ are

$K_{1}=\frac{\alpha\left[\left(\omega_{1}^{2}+\lambda_{2}^{2}\right) \cos \beta+2 \omega_{1} \sin \gamma\right]}{\omega_{1}\left(\lambda_{2}^{2}-\lambda_{1}^{2}\right)}$

$K_{2}=\frac{\alpha \lambda_{1}}{\omega_{1}{ }^{2} \omega_{2}}\left[\frac{2 \lambda_{2}{ }^{2} \sin \beta-\left(\omega_{1}{ }^{2}+\lambda_{2}{ }^{2}\right) \omega_{2} \cos \gamma}{\lambda_{2}{ }^{2}-\lambda_{1}{ }^{2}}\right]$

$K_{3}=-\frac{\alpha\left[\left(\omega_{1}{ }^{2}+\lambda_{1}{ }^{2}\right) \cos \beta+2 \omega_{1} \sin \gamma\right]}{\omega_{1}\left(\lambda_{2}{ }^{2}-\lambda_{1}{ }^{2}\right)}$

$K_{4}=\frac{\alpha \lambda_{2}}{\omega_{1}{ }^{2} \omega_{2}}\left[\frac{\left(\omega_{1}{ }^{2}+\lambda_{1}{ }^{2}\right) \omega_{2} \cos \gamma-2 \lambda_{1}{ }^{2} \sin \beta}{\lambda_{2}{ }^{2}-\lambda_{1}{ }^{2}}\right]$

Typical trajectories are shown in figure 3 for $\beta, \gamma=$ $0^{\circ}, 45^{\circ}, 90^{\circ}, 135^{\circ}, 180^{\circ}, 225^{\circ}, 270^{\circ}$, and $315^{\circ}$. With $\alpha=1$, most of the trajectories lay within the bounds of the size of the paper available (figs. 3(a) to 3(h)). For those trajectories that exceeded these bounds, the drawings were rescaled by choosing $\alpha=0.5$; these trajectories are given in green (figs. 3(i) to $3(n))$.

\subsection{ENVELOPES}

For the trajectories drawn, there is an exterior bounding curve for each. In section 6.1, a geometric description is given that enables determination of this bounding curve. The equation for the bounding curve, found as an envelope, is given in section 6.2. In section 6.3, orbits of spaceship observatories are described. An approximation to the bounding curve had been known; in section 6.4, this approximation is compared to the envelope determined in section 6.2. From the analysis given in section 6.2, an inner region from which the trajectory may be excluded can be determined; this is done in section 6.5. It was observed that, for all the trajectories drawn, $J_{2} \geqslant J_{1}$. The question thus arises: Is this always true or are there initial conditions for which $J_{1}>J_{2}$ ? This is answered affirmatively in section 6.6 and trajectories for a select set of initial conditions are drawn.

\subsection{A Geometric Description}

Consider a ray emanating from the libration point and the intersections of the trajectory with it. If the ray is referenced by an angle $\theta$, let $r(\theta)$ be the least upper bound of all the intersections. Then, $r=r(\theta)$ is the bounding curve for the trajectory.

On a fixed ray $r$, the distances from the libration point $x=y=0$ to successive intersections with the trajectory seem randomly generated. To proceed directly from this sequence of distances to their least upper bound seems hopeless; an alternate description is needed.

Consider a particle $P$ moving according to equations (4a) and (4b) where the $K_{i}$ are given by equations (5a) to (5d) for the initial conditions $\left(x_{0}, y_{0}\right)$, $\left(\dot{x}_{0}, \dot{y}_{0}\right)$. At $t=0$, when $P$ is launched, a spaceship $S_{1}$ is also launched. In describing the motion of $S_{1}$, whose equations of motion will also be equations (4a) and (4b), $K_{1}^{*}, K_{2}^{*}, K_{3}^{*}$, and $K_{4}^{*}$ shall be used to denote the coefficients. The initial conditions for $S_{1}$ will be represented by $\left(x_{0}^{*}, y_{0}^{*}\right),\left(\dot{x}_{0}^{*}, \dot{y}_{0}^{*}\right)$. The space- 
ship $S_{1}$ is launched so that

$$
\dot{y}_{o}^{*}=-\frac{\left(\omega_{1}^{2}+\lambda_{1}^{2}\right) x_{o}^{*}}{2}
$$

and

$$
y_{o}^{*}=\frac{\left(\omega_{1}^{2}+\lambda_{1}^{2}\right) \dot{x}_{o}^{*}}{2 \lambda_{1}^{2}}
$$

i.e., the initial conditions for spaceship $S_{1}$ are chosen so as to make $K_{3}^{*}=K_{4}^{*}=0$.

Under these conditions, the trajectory of the spaceship $S_{1}$ is given by

$$
\begin{aligned}
& x^{*}=K_{1}^{*} \cos \lambda_{1} t+K_{2}^{*} \sin \lambda_{1} t \\
& y^{*}=M_{1}\left(K_{2}^{*} \cos \lambda_{1} t-K_{1}^{*} \sin \lambda_{1} t\right)
\end{aligned}
$$

where $K_{1}^{*}=x_{o}^{*}$ and $K_{2}^{*}=\dot{x}_{0}^{*} / \lambda_{1}$. If $x_{o}^{*}$ and $\dot{x}_{o}^{*}$ are chosen as $x_{o}^{*}=K_{1}$ and $\dot{x}_{o}^{*}=\lambda_{1} K_{2}$, then $K_{1}^{*} \equiv K_{1}$ and $K_{2}^{*} \equiv K_{2}$. Thus, for appropriately chosen initial conditions, the trajectory of spaceship $S_{1}$ is the ellipse $E_{1}$

$$
\begin{aligned}
x^{*} & =K_{1} \cos \lambda_{1} t+K_{2} \sin \lambda_{1} t \\
& =J_{1} \cos \left(\lambda_{1} t-\phi_{1}\right) \\
y^{*} & =M_{1}\left(K_{2} \cos \lambda_{1} t-K_{1} \sin \lambda_{1} t\right) \\
& =M_{1} J_{1} \sin \left(\lambda_{1} t-\phi_{1}\right)
\end{aligned}
$$

As viewed by an observer on $S_{1}$, the particle $P$ describes the ellipse $E_{3}$

$$
\begin{aligned}
\bar{x} & =K_{3} \cos \lambda_{2} t+K_{4} \sin \lambda_{2} t \\
& =J_{2} \cos \left(\lambda_{2} t-\phi_{2}\right) \\
\bar{y} & =M_{2}\left(K_{4} \cos \lambda_{2} t-K_{3} \sin \lambda_{2} t\right) \\
& =M_{2} J_{2} \sin \left(\lambda_{2} t-\phi_{2}\right)
\end{aligned}
$$

The semi-major and semi-minor axes for $E_{1}$ are $J_{1}$ and $M_{1} J_{1}$, respectively; for $E_{3}$, they are $J_{2}$ and $M_{2} J_{2}$, respectively. An observer fixed in the $(x, y)$ system and looking at $S_{1}$ and $P$ can visualize the ellipse $E_{3}$ being attached to $S_{1}$ and moving with it. The center of $E_{3}$ is at $S_{1}$ and the axes are parallel to the axes of $E_{1}$, as shown in the following sketch.

The ellipse $E_{3}$ rides on the ellipse $E_{1}$

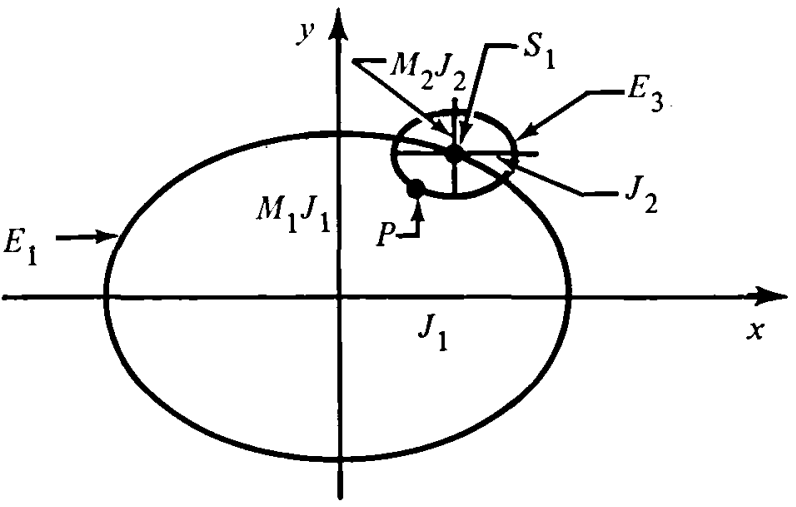

Again, consider the particle $P$ moving according to equations (4a) and (4b) where the $K_{i}$ are given by equations (5a) to (5d) for initial conditions $\left(x_{0}, y_{0}\right)$, $\left(\dot{x}_{o} \dot{y}_{o}\right)$. At $t=0$, when $P$ is launched, a second spaceship $S_{2}$ is also launched. In describing the motion of $S_{2}$, whose equations of motion shall also be equations (4a) and (4b), $K_{1}^{* *}, K_{2}^{* *}, K_{3}^{* *}$, and $K_{4}^{* *}$ shall be used to denote the coefficients. The initial conditions for $S_{2}$ will be represented by $\left(x_{o}^{* *}, y_{o}^{* *}\right),\left(\dot{x}_{o}^{* *}, \dot{y}_{o}^{* *}\right)$. The 
spaceship $S_{2}$ is launched so that

$$
\dot{y}_{o}^{* *}=-\frac{\left(\omega_{1}^{2}+\lambda_{2}^{2}\right) x_{o}^{* *}}{2}
$$

and

$$
y_{o}^{* *}=\frac{\left(\omega_{1}^{2}+\lambda_{2}^{2}\right) \dot{x}_{o}^{* *}}{2 \lambda_{2}^{2}}
$$

i.e., the initial conditions for spaceship $S_{2}$ are chosen so as to make $K_{1}^{* *}=K_{2}^{* *}=0$. The trajectory for spaceship $S_{2}$ is given by the equations

$$
\begin{aligned}
& x^{* *}=K_{3}^{* *} \cos \lambda_{2} t+K_{4}^{* *} \sin \lambda_{2} t \\
& y^{* *}=M_{2}\left(K_{4}^{* *} \cos \lambda_{2} t-K_{3}^{* *} \sin \lambda_{2} t\right)
\end{aligned}
$$

where $K_{3}^{* *}=x_{o}^{* *}$ and $K_{4}^{* *}=\dot{x}_{o}^{* *} / \lambda_{2}$. If $x_{o}^{* *}$ and $\dot{x}_{o}^{* *}$ are chosen as $x_{0}^{* *}=K_{3}$ and $\dot{x}_{o}^{* *}=\lambda_{2} K_{4}$, then $K_{3}^{* *} \equiv$ $K_{3}$ and $K_{4}^{* *} \equiv K_{4}$. Thus, for appropriately chosen initial conditions, the trajectory of spaceship $S_{2}$ is the ellipse $E_{2}$

$$
\begin{aligned}
x^{* *} & =K_{3} \cos \lambda_{2} t+K_{4} \sin \lambda_{2} t \\
& =J_{2} \cos \left(\lambda_{2} t-\phi_{2}\right) \\
y^{* *} & =M_{2}\left(K_{4} \cos \lambda_{2} t-K_{3} \sin \lambda_{2} t\right) \\
& =M_{2} J_{2} \sin \left(\lambda_{2} t-\phi_{2}\right)
\end{aligned}
$$

$P$ describes the ellipse $E_{4}$

$$
\begin{aligned}
\overline{\bar{x}} & =K_{1} \cos \lambda_{1} t+K_{2} \sin \lambda_{1} t \\
& =J_{1} \cos \left(\lambda_{1} t-\phi_{1}\right) \\
\overline{\bar{y}} & =M_{1}\left(K_{2} \cos \lambda_{1} t-K_{1} \sin \lambda_{1} t\right) \\
& =M_{1} J_{1} \sin \left(\lambda_{1} t-\phi_{1}\right)
\end{aligned}
$$

The semi-major and semi-minor axes for $E_{2}$ are $J_{2}$ and $M_{2} J_{2}$, respectively; for $E_{4}$, they are $J_{1}$ and $M_{1} J_{1}$, respectively. The geometry for this situation is illustrated in the following sketch.

The ellipse $E_{4}$ rides on the ellipse $E_{2}$

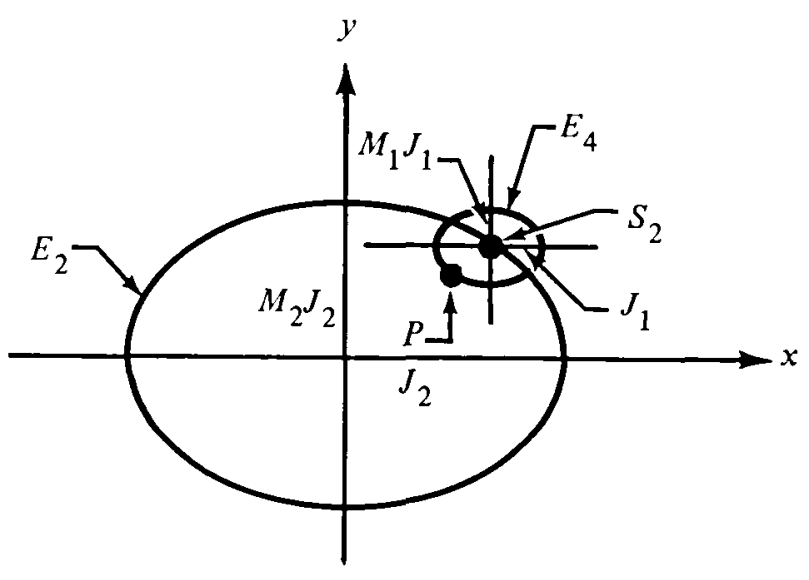

In the subsequent discussions, the description will be based on the image of the ellipse with the smaller semi-major axis riding on that spaceship whose trajectory has the larger semi-major axis. In effect, $J_{1}$ and $J_{2}$ are being compared, since $M_{1} \approx 0.49 \ldots$ and $M_{2} \approx 0.19 \ldots$ for $\mu=1 / 82.45$.

and, when viewed by an observer on $S_{2}$, the particle 


\subsection{The Equation of the Envelope}

When a fixed ray emanating from the origin intersects the smaller ellipse as it is being carried piggyback on the larger ellipse, the farthest point of intersection is an upper bound for all intersections of that ray and the trajectory. This farthest point of intersection can be found quantitatively.

Assume that $J_{1}>J_{2}$. The equation of the ellipse which the spaceship $S_{1}$ traverses is $x=J_{1} \cos \alpha$ and $y=M_{1} J_{1} \sin \alpha$, where $\alpha$, the eccentric angle, is considered a parameter. ${ }^{4}$ The equation of the smaller ellipse on whose perimeter the particle rides is

$$
\frac{\left(x-J_{1} \cos \alpha\right)^{2}}{J_{2}^{2}}+\frac{\left(y-M_{1} J_{1} \sin \alpha\right)^{2}}{M_{2}^{2} J_{2}^{2}}=1
$$

If the envelope of this one-parameter family of ellipses is determined, a bound for the trajectory will be found. To find the envelope, first differentiate equation (17) with respect to $\alpha$. This produces

$$
\frac{J_{1}}{J_{2}{ }^{2}}\left(x-J_{1} \cos \alpha\right) \sin \alpha-\frac{M_{1} J_{1}}{M_{2}{ }^{2} J_{2}{ }^{2}}\left(y-M_{1} J_{1} \sin \alpha\right) \cos \alpha=0
$$

which can be simplified to

$$
\left(x-J_{1} \cos \alpha\right) \sin \alpha-\frac{M_{1}}{M_{2}{ }^{2}}\left(y-M_{1} J_{1} \sin \alpha\right) \cos \alpha=0
$$

It remains to solve equations (17) and (18) for $x$ and $y$. Solve equation (18) for $\left(x-J_{1} \cos \alpha\right)$ and substitute this into equation (17). The resulting equation can be solved for $y$, there are two solutions:

$$
y^{\#}=M_{1} J_{1} \sin \alpha \pm \frac{M_{2}{ }^{2} J_{2} \sin \alpha}{\sqrt{M_{1}{ }^{2} \cos ^{2} \alpha+M_{2}{ }^{2} \sin ^{2} \alpha}}
$$

When these two values of $y$ are substituted into equation (18), two values of $x$ are determined.

$$
x^{\#}=J_{1} \cos \alpha \pm \frac{M_{1} J_{2} \cos \alpha}{\sqrt{M_{1}^{2} \cos ^{2} \alpha+M_{2}^{2} \sin ^{2} \alpha}}
$$

It is important to remember that the plus signs go together as do the minus signs. Equations (19a) and (19b) are the parametric equations for the envelope. Using the plus signs, the bounding curve is drawn in blue in figures 1 to 3 .

The analysis for $J_{2} \geqslant J_{1}$ parallels that given above and leads to the equations ${ }^{5}$

$$
\begin{aligned}
& x^{\#}=J_{2} \cos \alpha \pm \frac{M_{2} J_{1} \cos \alpha}{\sqrt{M_{2}{ }^{2} \cos ^{2} \alpha+M_{1}{ }^{2} \sin ^{2} \alpha}} \\
& y^{\#}=M_{2} J_{2} \sin \alpha \pm \frac{M_{1}{ }^{2} J_{1} \sin \alpha}{\sqrt{M_{2}{ }^{2} \cos ^{2} \alpha+M_{1}{ }^{2} \sin ^{2} \alpha}}
\end{aligned}
$$

\subsection{The Orbit of the Spaceship}

Each spaceship, because of the special way the initial conditions were chosen (i.e., for $S_{1}, J_{2}=0$; for $S_{2}, J_{1}=0$ ), has a periodic orbit, an ellipse. These elliptic orbits are described in reference 2 (pp. 258-261).

\subsection{An Approximation to the Envelope}

An approximation to the envelope developed in section 6.2 can be obtained by arbitrarily assuming the envelope to be an ellipse with semi-major axis $J_{1}+J_{2}$ and semi-minor axis $M_{1} J_{1}+M_{2} J_{2}$ (ref. 2, p. 283). A comparison of this approximate bound and the bound found in section 6.2 is shown in figure 4 . The bounding curve is drawn in blue and the ellipse in red. At the scale in which the figures are drawn, the approximation is remarkable.

\footnotetext{
${ }^{5}$ Note that, in this case, $\alpha=\lambda_{2} t-\phi_{2}$.
}

\footnotetext{
${ }^{4}$ Note that $\alpha=\lambda_{1} t-\phi_{1}$.
} 


\subsection{An Inner Bounding Curve}

In section 6.2 , an outer bounding curve was obtained when the plus sign was used in equations (19a) and (19b). From the geometry of a family of ellipses whose centers lie on a fixed ellipse, one suspects that, under the right conditions, there may be an inner region from which the trajectory is excluded. The existence of such an inner region depends on the relative magnitude of $J_{1}$ and $J_{2}$ and the relative magnitude of $M_{1} J_{1}$ and $M_{2} J_{2}$.

The case of $C=0$ is disposed of easily. From equation (16), it is found that

$$
J_{1}^{2}=\left(\frac{\lambda_{2}{ }^{2}-\omega_{2}{ }^{2} M_{2}{ }^{2}}{\omega_{2}{ }^{2} M_{1}{ }^{2}-\lambda_{1}{ }^{2}}\right) J_{2}{ }^{2}
$$

and for $\mu=1 / 82.45$,

$$
J_{1}=0.3515 .131989732791 J_{2}
$$

and

$$
\left(M_{1} J_{1}\right)=0.8878271396217024\left(M_{2} J_{2}\right)
$$

In this case (refer to the sketch on page 12), there is always an inner region from which the trajectory is excluded. Equations (19a) and (19b), when the minus sign is used, produce a curve that looks like the one shown in the following sketch.

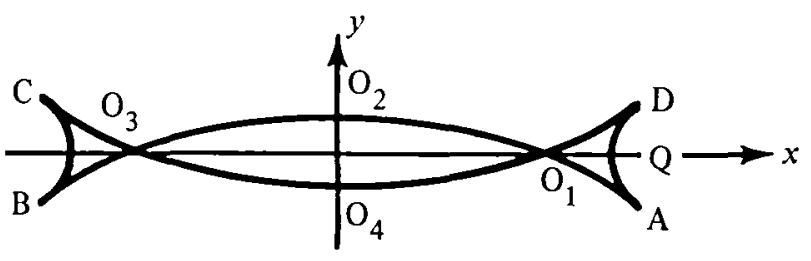

For the parameter $\alpha$ ( $\alpha$ going from 0 to $2 \pi$ ), the sequence to be followed in tracing the curve is $\mathrm{QAO}_{1} \mathrm{O}_{2} \mathrm{O}_{3} \mathrm{BCO}_{3} \mathrm{O}_{4} \mathrm{O}_{1} \mathrm{DQ}$. However, the two trian- gular regions, $\mathrm{AO}_{1} \mathrm{D}$ and $\mathrm{BO}_{3} \mathrm{C}$, are not part of the excluded region; the excluded region is bounded by $\mathrm{O}_{1} \mathrm{O}_{2} \mathrm{O}_{3} \mathrm{O}_{4}$. A typical situation is shown in figure 5 . In figure 6, the family of smaller ellipses is drawn in black, the trajectory in green, and the bounding curves in red.

For $C=+1$, there may be no inner excluded region, as can be seen in figure 7 . The inner envelope in this illustration, drawn in green, does not encompass a region from which the trajectory is excluded. In the next section, initial conditions are given for which there does exist an inner region from which the trajectory is excluded.

For $C=-1$, there will always be an inner excluded region that includes the Jacobi ellipse.

\subsection{On the Relative Magnitude of $J_{1}$ and $J_{2}$}

Equation (16) can be rewritten as

$$
J_{1}{ }^{2}=\frac{\left(\omega_{2}{ }^{2} M_{2}{ }^{2}-\lambda_{2}{ }^{2}\right)}{\left(\lambda_{1}{ }^{2}-\omega_{2}{ }^{2} M_{1}{ }^{2}\right)} J_{2}{ }^{2}+\frac{C}{\left(\lambda_{1}{ }^{2}-\omega_{2}{ }^{2} M_{1}{ }^{2}\right)}
$$

For $\mu=1 / 82.45$,

$J_{1}{ }^{2}=(0.1235615290524281) J_{2}{ }^{2}+(5.182189180103332) C$

For all the figures drawn thus far, $J_{1} \leqq J_{2}$; are there initial conditions for which $J_{1}>J_{2}$ ? If $C=0$,

$$
J_{1}=(0.3515131989732791) J_{2}
$$

and clearly $J_{1}<J_{2}$. Similarly, if $C=-1$,

$$
J_{1}<(0.3515131989732791) J_{2}
$$

Thus, if any trajectories are to be found for which $J_{1}>J_{2}$, they can only be among those for which $C=+1$.

It is not intended to exhibit samples of all trajectories for which $J_{1}>J_{2}$, only some of a special set. If $J_{1}$ 
$>J_{2}$, then $J_{1}{ }^{2}>J_{2}{ }^{2}$ and $K_{1}{ }^{2}+K_{2}{ }^{2}>K_{3}{ }^{2}+K_{4}{ }^{2}$. Using the parametric representation of equation (22) in the appendix,

$K_{1}=\frac{\left(\omega_{1}{ }^{2}+\lambda_{2}{ }^{2}\right) \cosh \alpha \sinh \beta+2 \omega_{1} \cosh \alpha \cosh \beta \sin \gamma}{\omega_{1}\left(\lambda_{2}{ }^{2}-\lambda_{1}{ }^{2}\right)}$

$K_{2}=\frac{\lambda_{1}}{\omega_{1}{ }^{2}}\left[\frac{2 \lambda_{2}{ }^{2} \sinh \alpha-\omega_{2}\left(\omega_{1}{ }^{2}+\lambda_{2}{ }^{2}\right) \cosh \alpha \cosh \beta \cos \gamma}{\omega_{2}\left(\lambda_{2}{ }^{2}-\lambda_{1}{ }^{2}\right)}\right]$

$K_{3}=-\frac{\left(\omega_{1}{ }^{2}+\lambda_{1}{ }^{2}\right) \cosh \alpha \sinh \beta+2 \omega_{1} \cosh \alpha \cosh \beta \sin \gamma}{\omega_{1}\left(\lambda_{2}{ }^{2}-\lambda_{1}{ }^{2}\right)}$

$K_{4}=\frac{\lambda_{2}}{\omega_{1}{ }^{2}}\left[\frac{\omega_{2}\left(\omega_{1}{ }^{2}+\lambda_{1}{ }^{2}\right) \cosh \alpha \cosh \beta \cos \gamma-2 \lambda_{1}{ }^{2} \sinh \alpha}{\omega_{2}\left(\lambda_{2}{ }^{2}-\lambda_{1}{ }^{2}\right)}\right]$

Special values are chosen for $\alpha$ and $\gamma ; \alpha=0, \gamma=$ $90^{\circ}$. For these values,

$$
\begin{aligned}
& K_{1}=\frac{\left(\omega_{1}^{2}+\lambda_{2}{ }^{2}\right) \sinh \beta+2 \omega_{1} \cosh \beta}{\omega_{1}\left(\lambda_{2}{ }^{2}-\lambda_{1}{ }^{2}\right)} \\
& K_{2}=0 \\
& K_{3}=-\frac{\left(\omega_{1}^{2}+\lambda_{1}{ }^{2}\right) \sinh \beta+2 \omega_{1} \cosh \beta}{\omega_{1}\left(\lambda_{2}{ }^{2}-\lambda_{1}{ }^{2}\right)} \\
& K_{4}=0
\end{aligned}
$$

Thus, $J_{1}>J_{2}$ implies that $\left|K_{1}\right|>\left|K_{3}\right|$.

\section{Case 1}

$$
\begin{aligned}
& {\left[\left(\omega_{1}^{2}+\lambda_{2}^{2}\right) \sinh \beta+2 \omega_{1} \cosh \beta\right]>} \\
& -\left[\left(\omega_{1}^{2}+\lambda_{1}^{2}\right) \sinh \beta+2 \omega_{1} \cosh \beta\right]
\end{aligned}
$$

This implies that

$$
\tanh \beta>-\frac{4 \omega_{1}}{2 \omega_{1}{ }^{2}+\lambda_{1}{ }^{2}+\lambda_{2}{ }^{2}}
$$

or that $\beta>-0.7343384813667741$.

Case 2

$$
\begin{aligned}
& {\left[\left(\omega_{1}^{2}+\lambda_{2}^{2}\right) \sinh \beta+2 \omega_{1} \cosh \beta\right]>} \\
& \left.\qquad\left(\omega_{1}{ }^{2}+\lambda_{1}{ }^{2}\right) \sinh \beta+2 \omega_{1} \cosh \beta\right]
\end{aligned}
$$

This implies that

$$
\left(\lambda_{2}^{2}-\lambda_{1}^{2}\right) \sinh \beta>0
$$

or, since $\left(\lambda_{2}{ }^{2}-\lambda_{1}{ }^{2}\right)<0$, that $\sinh \beta<0$. However, $\sinh \beta<0$ for $\beta<0$. Therefore, we find that $J_{1}>J_{2}$ for $\alpha=0,-0.73<\beta<0, \gamma=90^{\circ}$. Trajectories for $\beta=-0.1$ through -0.7 are given in figure 8 . The shape of the interior excluded region in figure 8 , as shown in the following sketch, is similar to that of the sketch shown in section 6.5 rotated $90^{\circ}$. 


\subsection{CONCLUDING REMARKS}

The derivation of the finite equations of motion from the differential equations that describe the motion of a point particle in the neighborhood of a triangular libration point $\left(L_{4}\right.$ or $\left.L_{5}\right)$ in the linearized, restricted problem of three bodies is well known. From these equations, three integral invariants of the motion were derived; the Jacobi integral is expressed linearly in terms of two of these.

The trajectories for varied initial conditions were drawn. The motion of a point particle as it traversed its trajectory was described in elementary geometric terms.

Each trajectory has an exterior boundary curve; its equation was found. An approximation to this boundary curve had been known; it is a well-defined ellipse. For different initial conditions, the boundary curve and the ellipse were compared graphically; the agreement seems remarkable. For certain initial conditions, there is an interior region from which the trajectory is excluded; the equation of the boundary of this region was found.

Lyndon B. Johnson Space Center

National Aeronautics and Space Administration Houston, Texas, September 28, 1979 953-36-00-00-72

\subsection{REFERENCES}

1. Moulton, F. R.: An Introduction to Celestial Mechanics. The MacMillan Co. (New York), 1939, pp. 277-281 and 298-307.

2. Szebehely, Victor: Theory of Orbits. Academic Press (New York), 1967. 


\section{Appendix \\ Parameterizations for the Jacobi Ellipse}

A convenient method for choosing the initial values of the position and velocity of the particle can be obtained from the Jacobi integral:

$$
\dot{x}_{o}^{2}+\dot{y}_{o}^{2}-\omega_{1}^{2} x_{o}^{2}-\omega_{2}^{2} y_{o}^{2}=C
$$

If $C=-1$, equation (20) can be parameterized by

$$
\begin{aligned}
& x_{o}=\frac{1}{\omega_{1}} \cosh \alpha \cosh \beta \cos \gamma \\
& y_{o}=\frac{1}{\omega_{2}} \cosh \alpha \cosh \beta \sin \gamma \\
& \dot{x}_{o}=\cosh \alpha \sinh \beta \\
& \dot{y}_{o}=\sinh \alpha
\end{aligned}
$$

For the rest trajectories (sec. 5.1), $\alpha=\beta=0, \gamma$ arbitrary. If the initial velocity is not zero, $\alpha$ and $\beta$ can be determined from equations (21c) and (21d) and $\gamma$ from equations (21a) and (21b).

If $C=+1$, equation (20) can be parameterized by

$$
\begin{aligned}
& x_{o}=\frac{1}{\omega_{1}} \cosh \alpha \sinh \beta \\
& y_{o}=\frac{1}{\omega_{2}} \sinh \alpha \\
& \dot{x}_{o}=\cosh \alpha \cosh \beta \cos \gamma \\
& \dot{y}_{o}=\cosh \alpha \cosh \beta \sin \gamma
\end{aligned}
$$

For trajectories that start at the libration point $\left(x_{o}=\right.$ $\left.y_{0}=0\right), \alpha=\beta=0, \gamma$ arbitrary. If the initial position is different from the origin, $\alpha$ and $\beta$ can be determined from equations (22a) and (22b) and $\gamma$ from equations $(22 \mathrm{c})$ and $(22 \mathrm{~d})$.

If $C=0$, equation (20) can be parameterized by

$$
\begin{aligned}
& x_{o}=\frac{1}{\omega_{1}} \alpha \cos \beta \\
& y_{o}=\frac{1}{\omega_{2}} \alpha \sin \beta \\
& \dot{x}_{o}=\alpha \cos \gamma \\
& \dot{y}_{o}=\alpha \cos \gamma
\end{aligned}
$$

Included among these trajectories is the solution corresponding to $\alpha=0$; i.e., the particle is at the libration point with zero initial velocity and remains there indefinitely. Leaving this solution aside, the parameterization is as given above. 


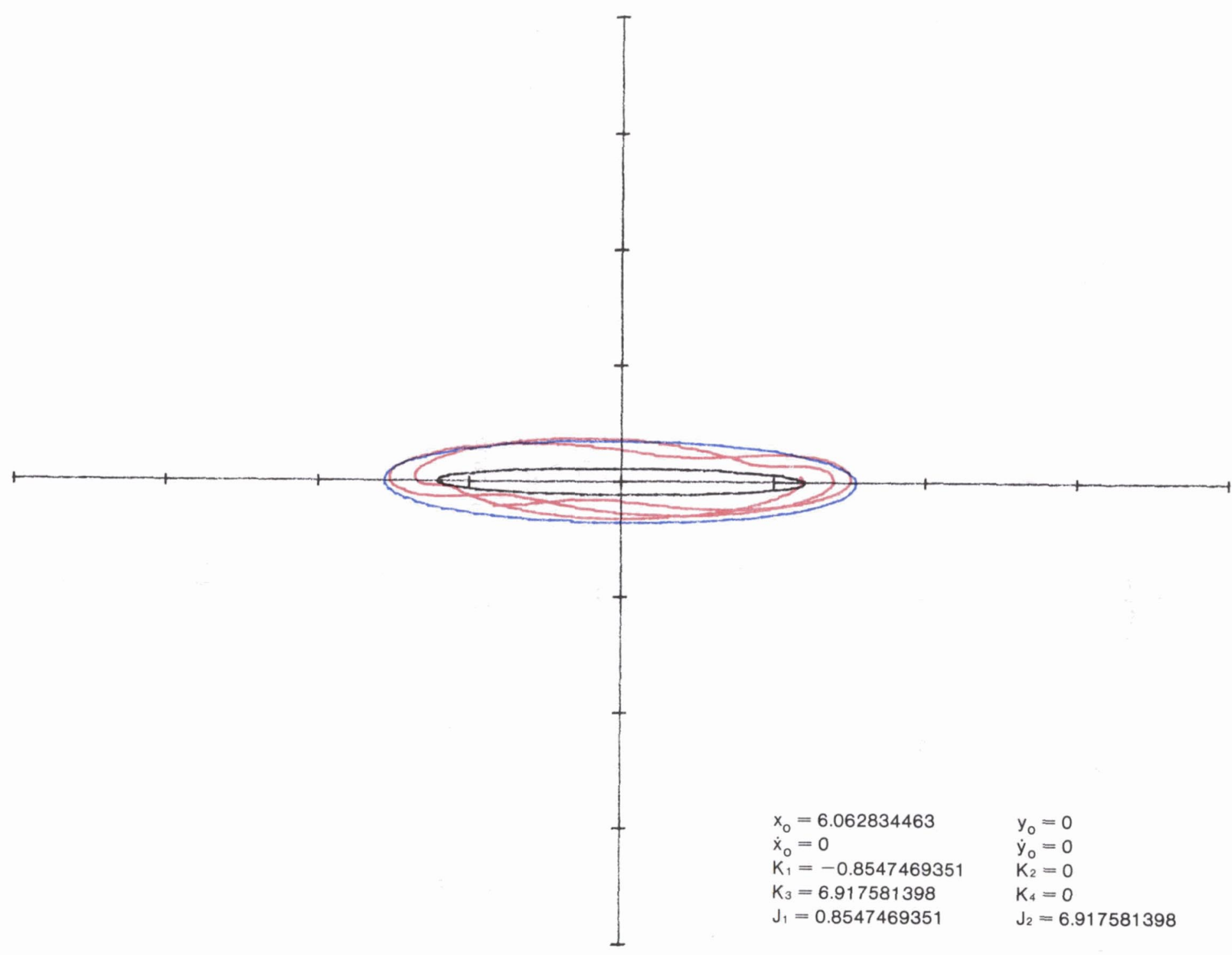

(a) $\alpha=0, \beta=0, \gamma=0^{\circ}$.

FIGURE 1.-Typical trajectories for which $C=-1$. 


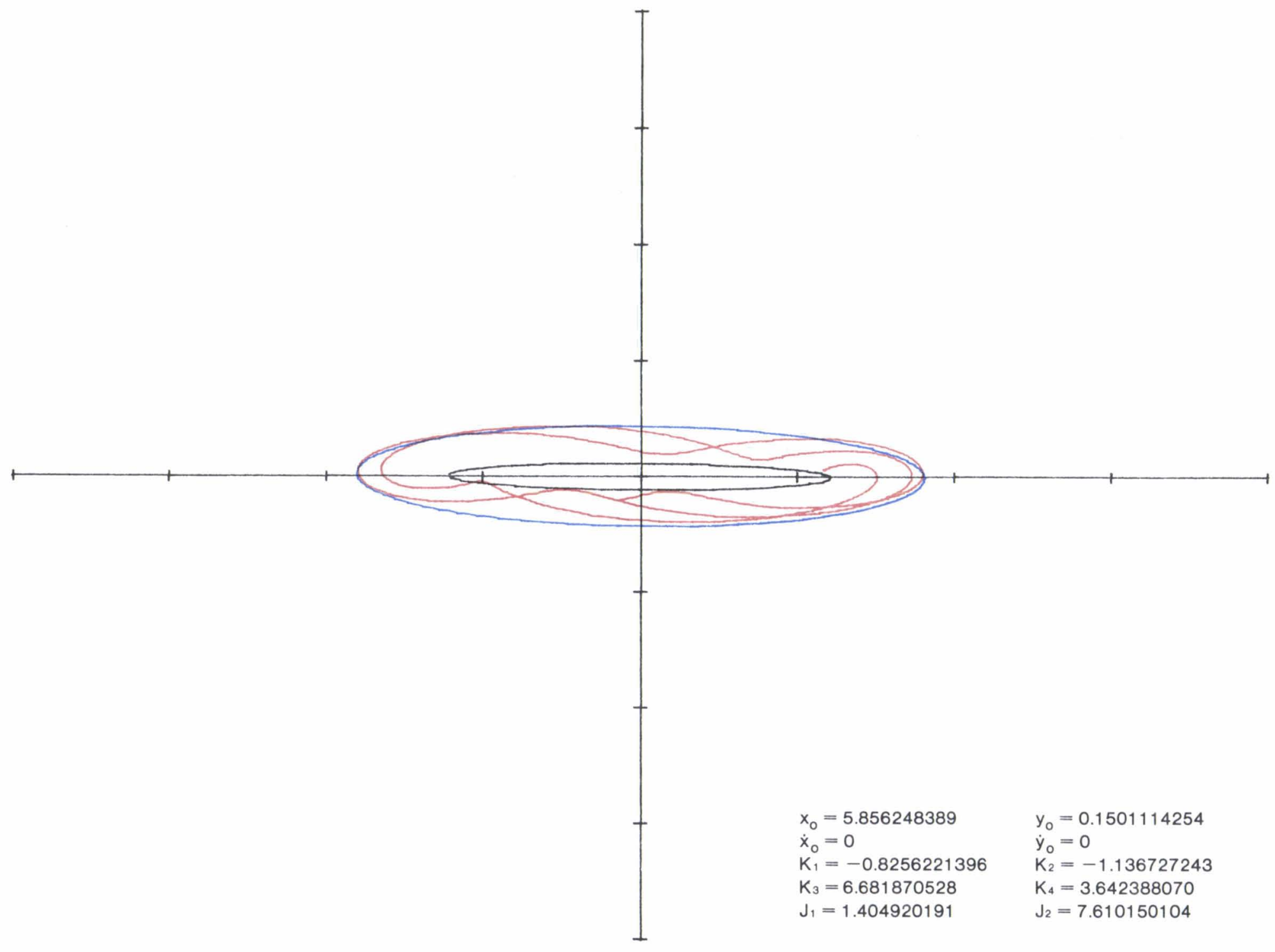

(b) $\alpha=0, \beta=0, \gamma=15^{\circ}$. 


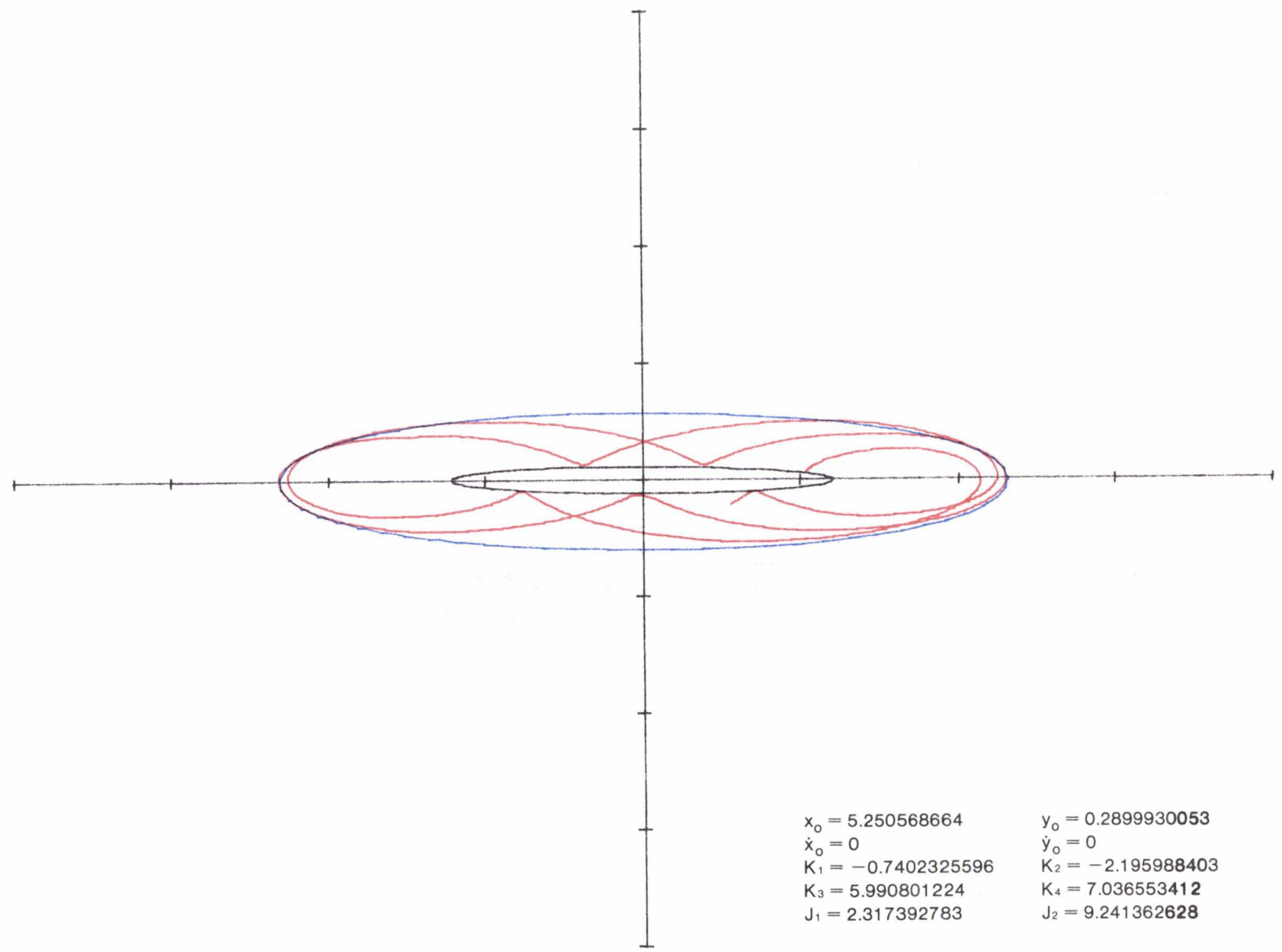

(c) $\alpha=\mathbf{0}, \beta=0, \gamma=30^{\circ}$.

FIGURE 1.-Continued. 


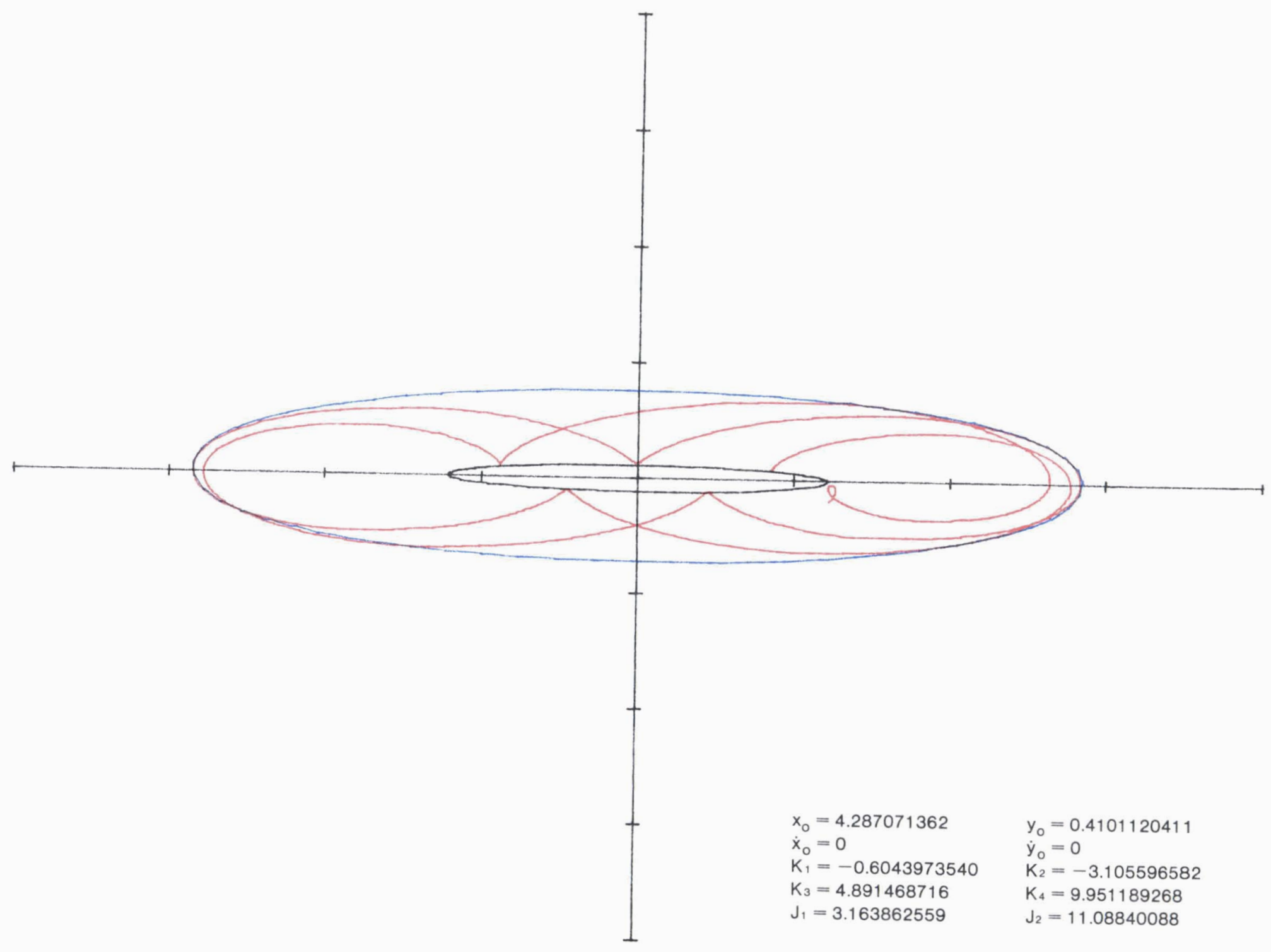

(d) $\alpha=0, \beta=0, \gamma=45^{\circ}$. 


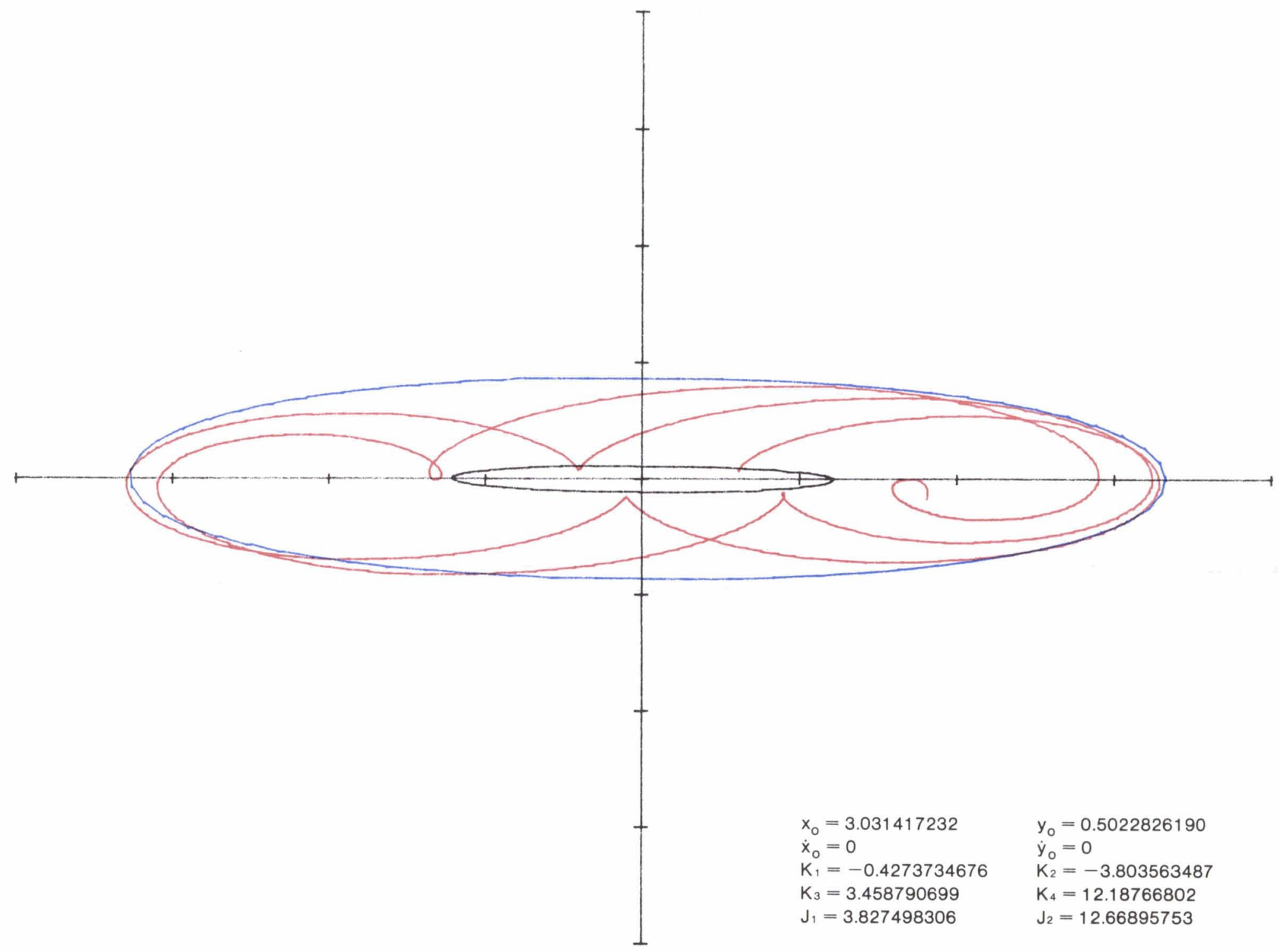

(e) $\alpha=0, \beta=0, \gamma=60^{\circ}$.

FIGURE 1.-Continued. 


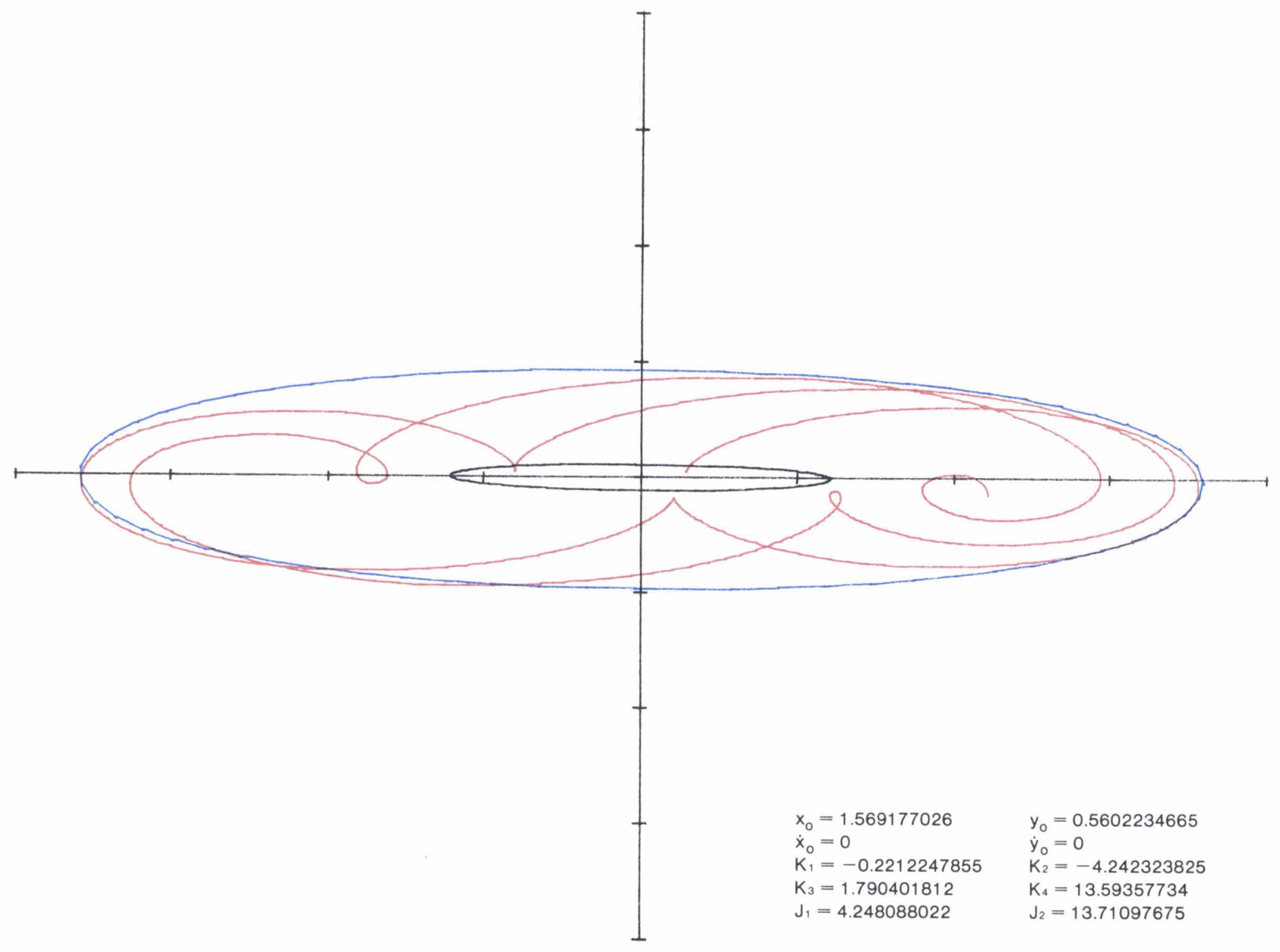

(f) $\alpha=0, \beta=0, \gamma=75^{\circ}$.

FIGURE 1.-Continued. 


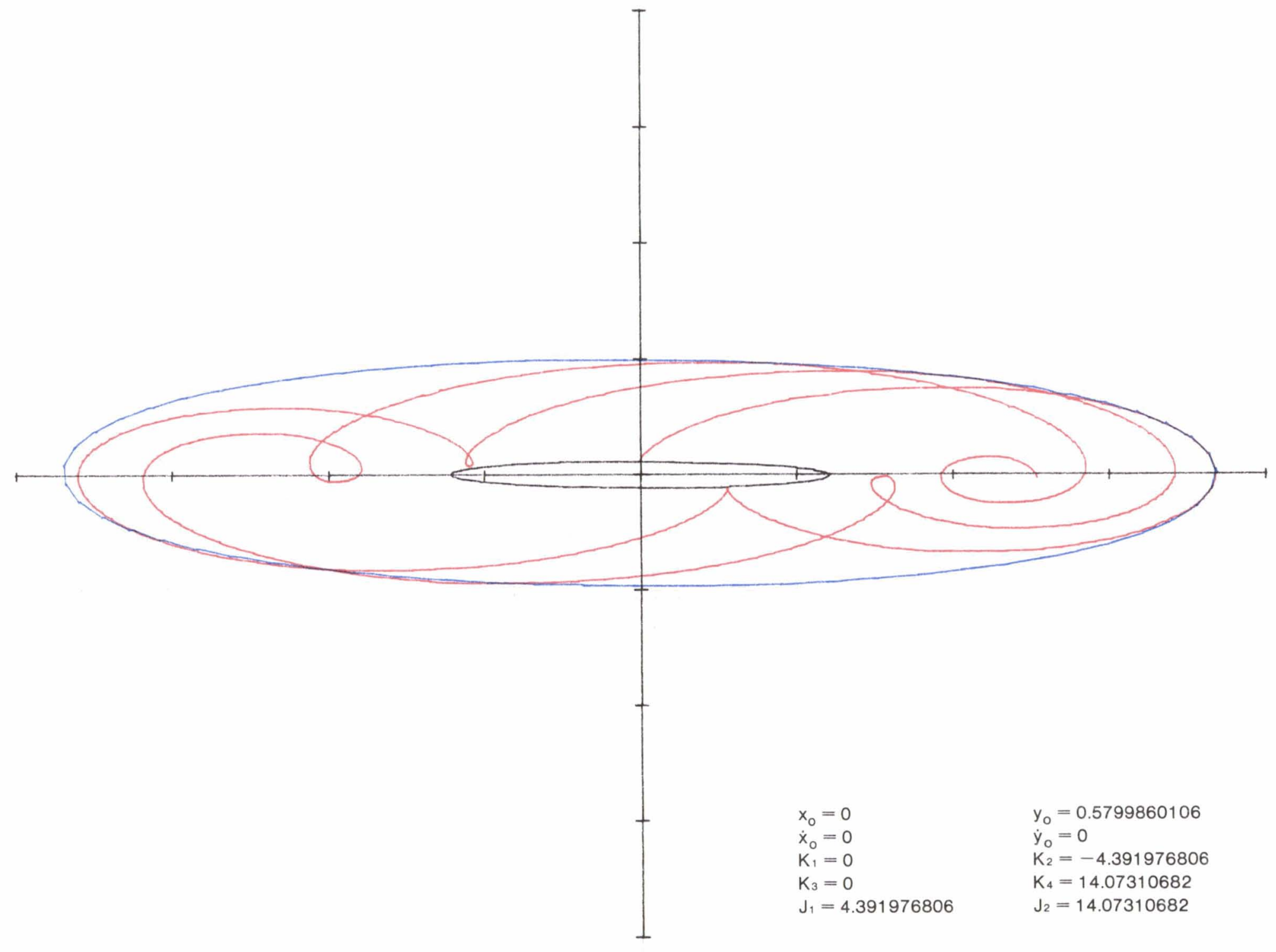

(g) $\alpha=\mathbf{0}, \beta=0, \gamma=90^{\circ}$. 


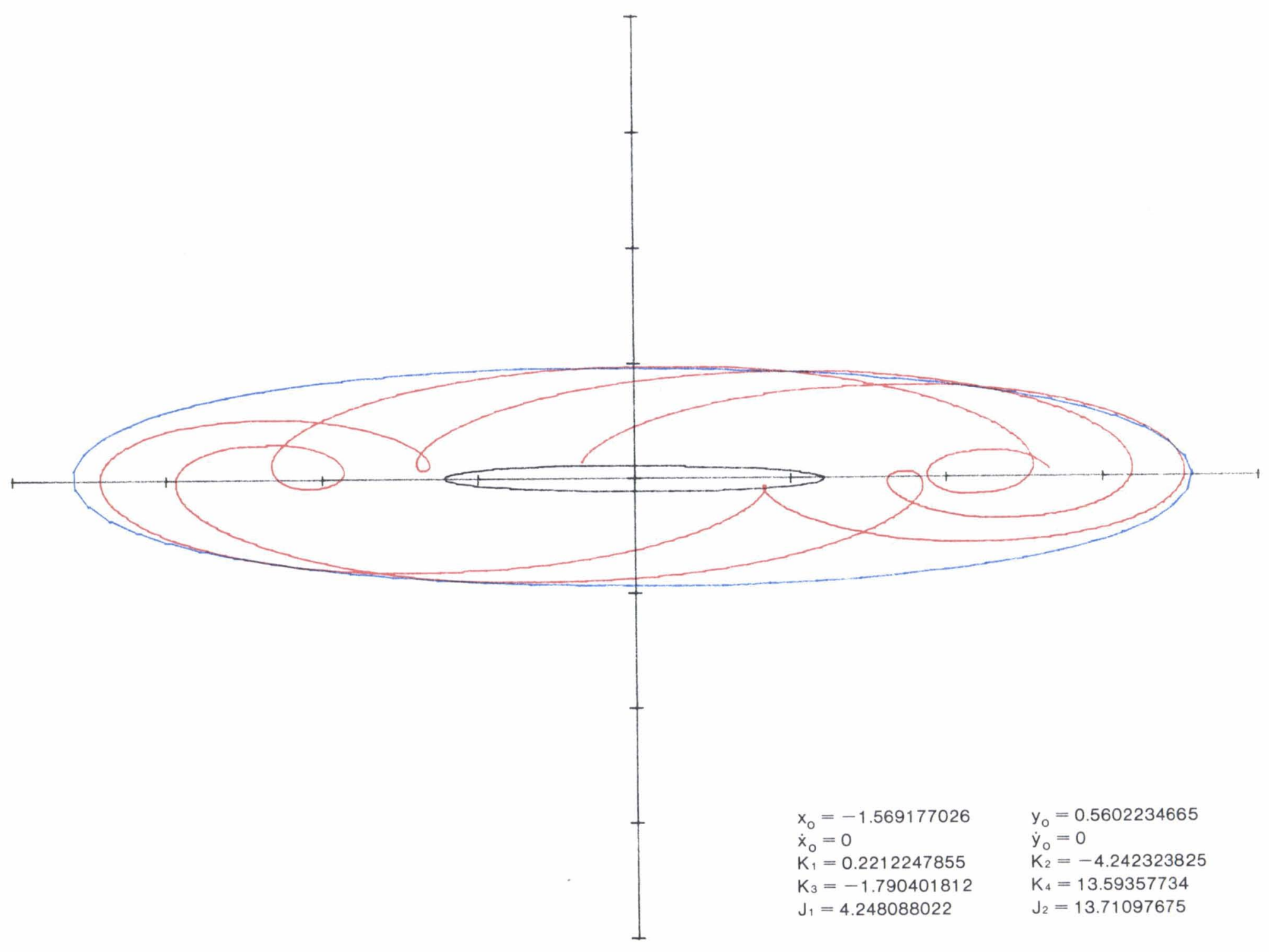

(h) $\alpha=0, \beta=0, \gamma=105^{\circ}$. 


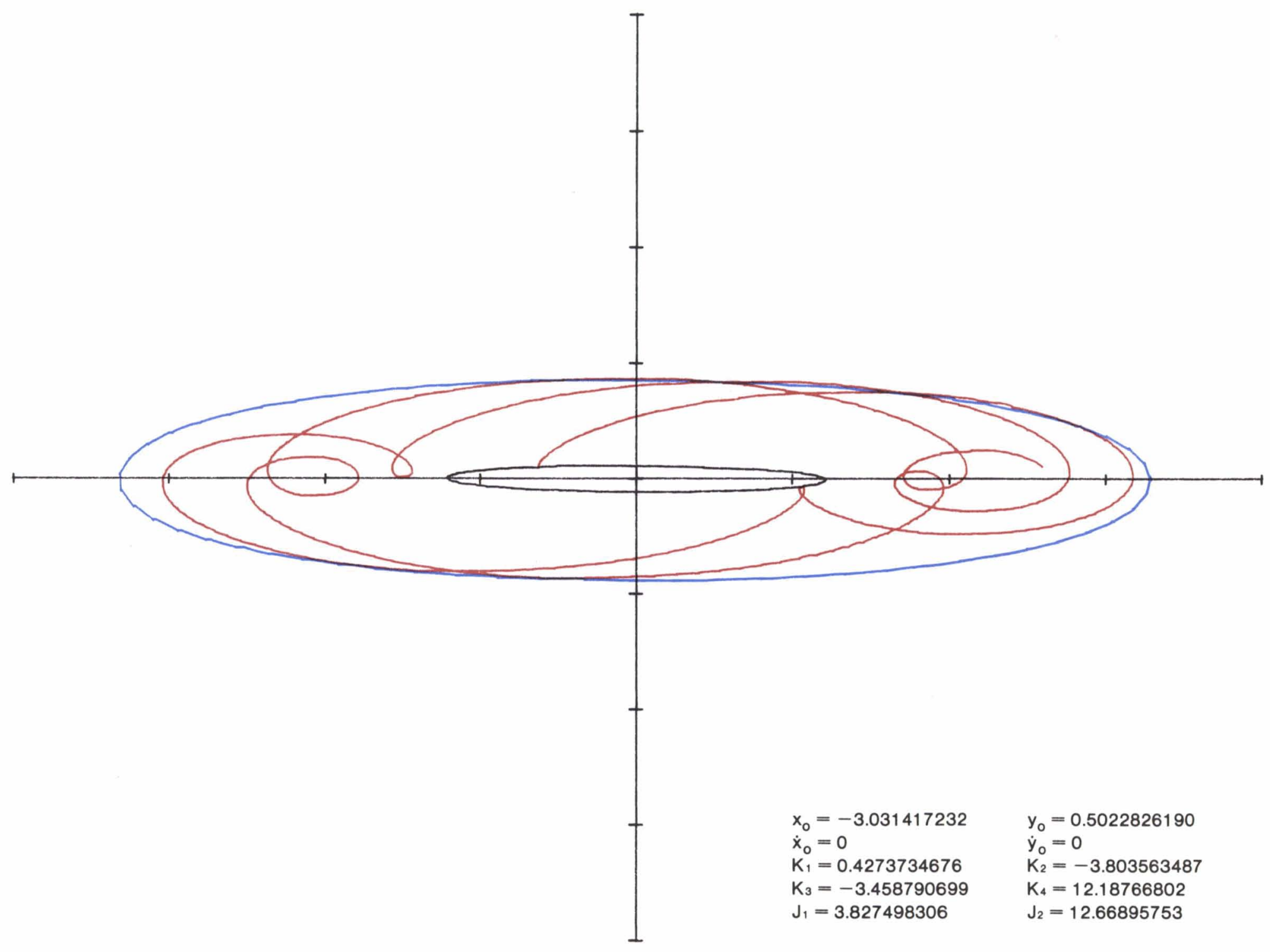

(i) $\alpha=0, \beta=0, \gamma=120^{\circ}$.

FIGURE 1.-Continued. 


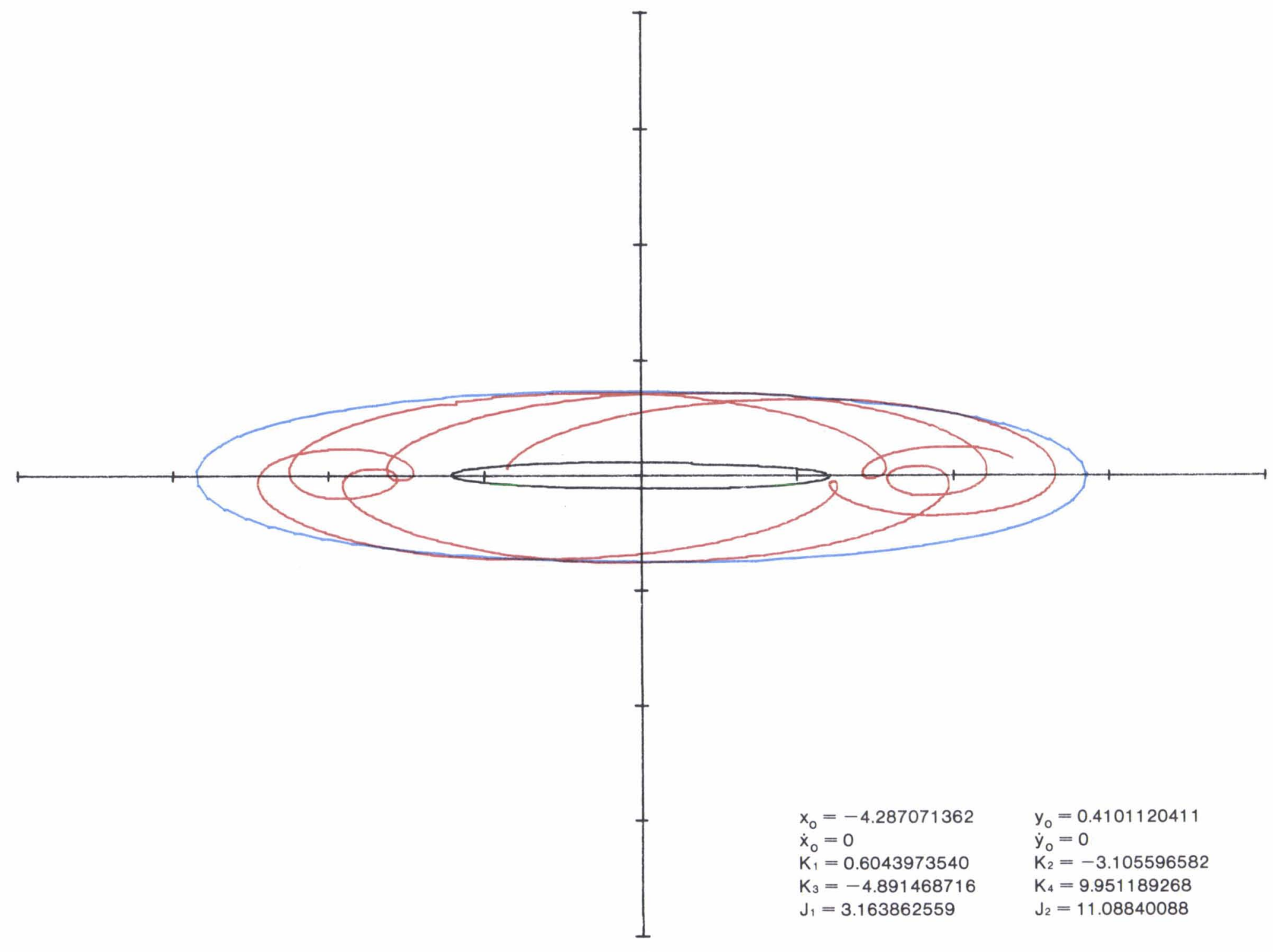

(j) $\alpha=\mathbf{0}, \beta=0, \gamma=135^{\circ}$. 


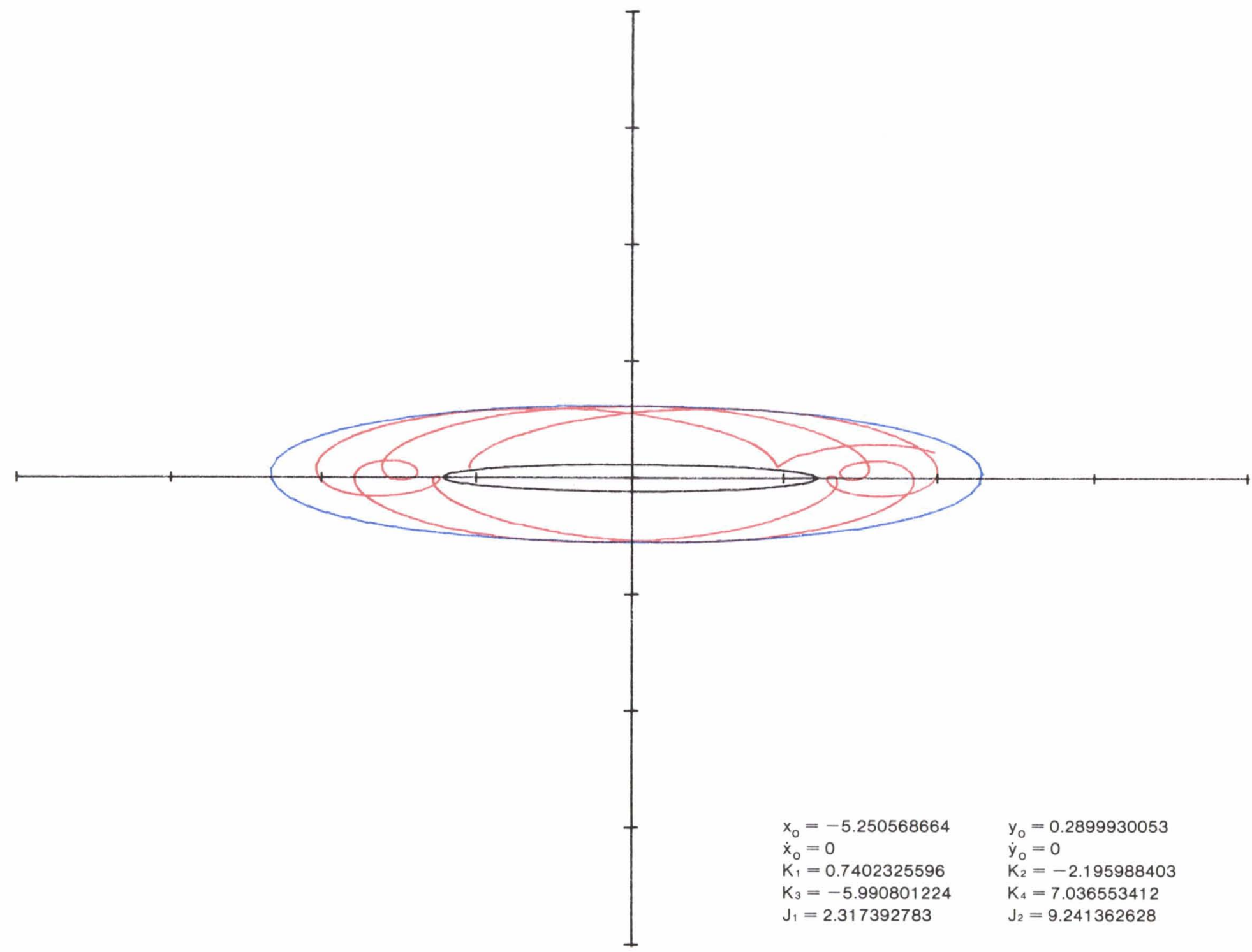

(k) $\alpha=0, \beta=0, \gamma=150^{\circ}$.

FIGURE 1.-Continued 


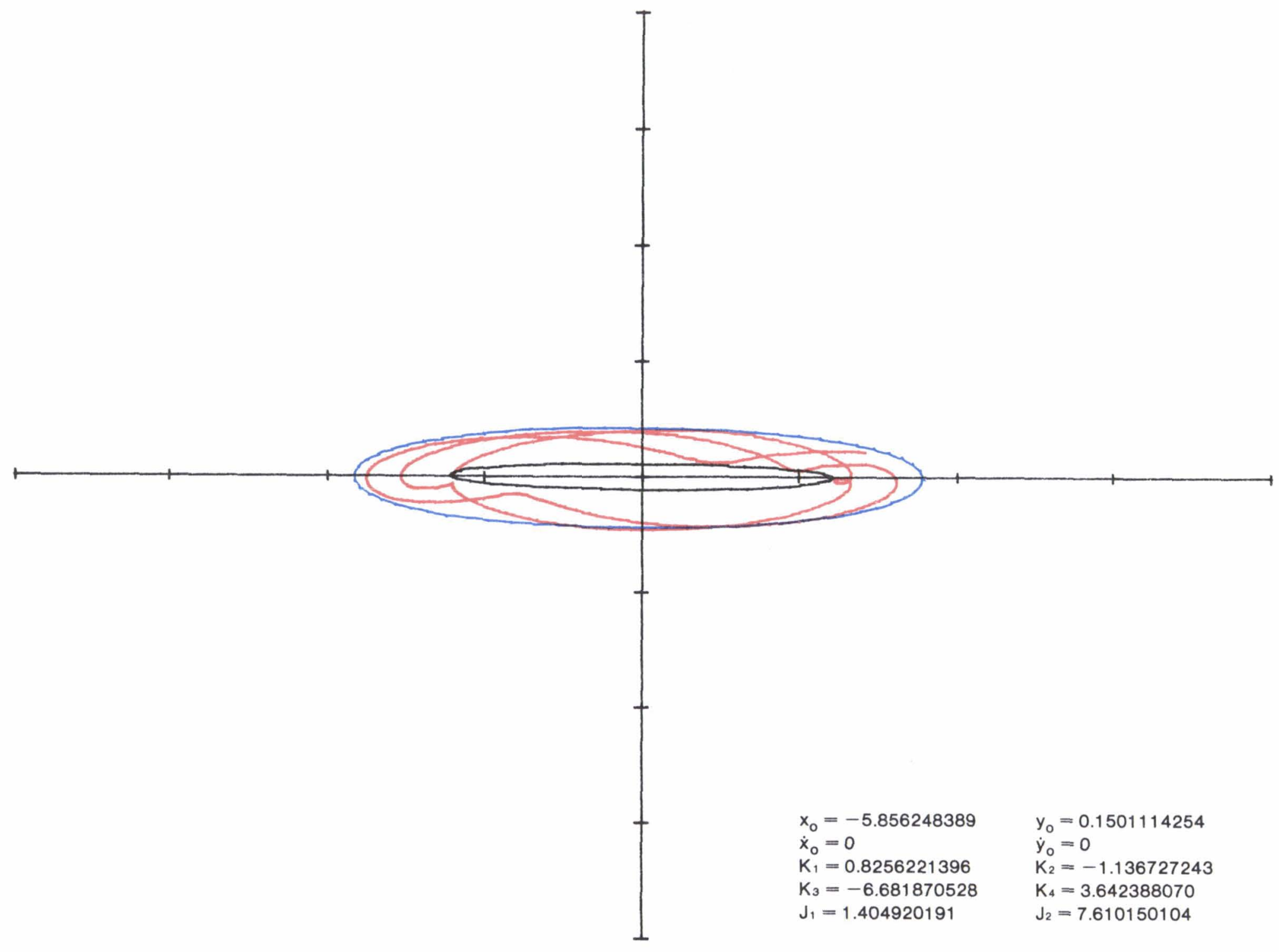

(l) $\alpha=0, \beta=0, \gamma=165^{\circ}$.

FIGURE 1.-Continued. 


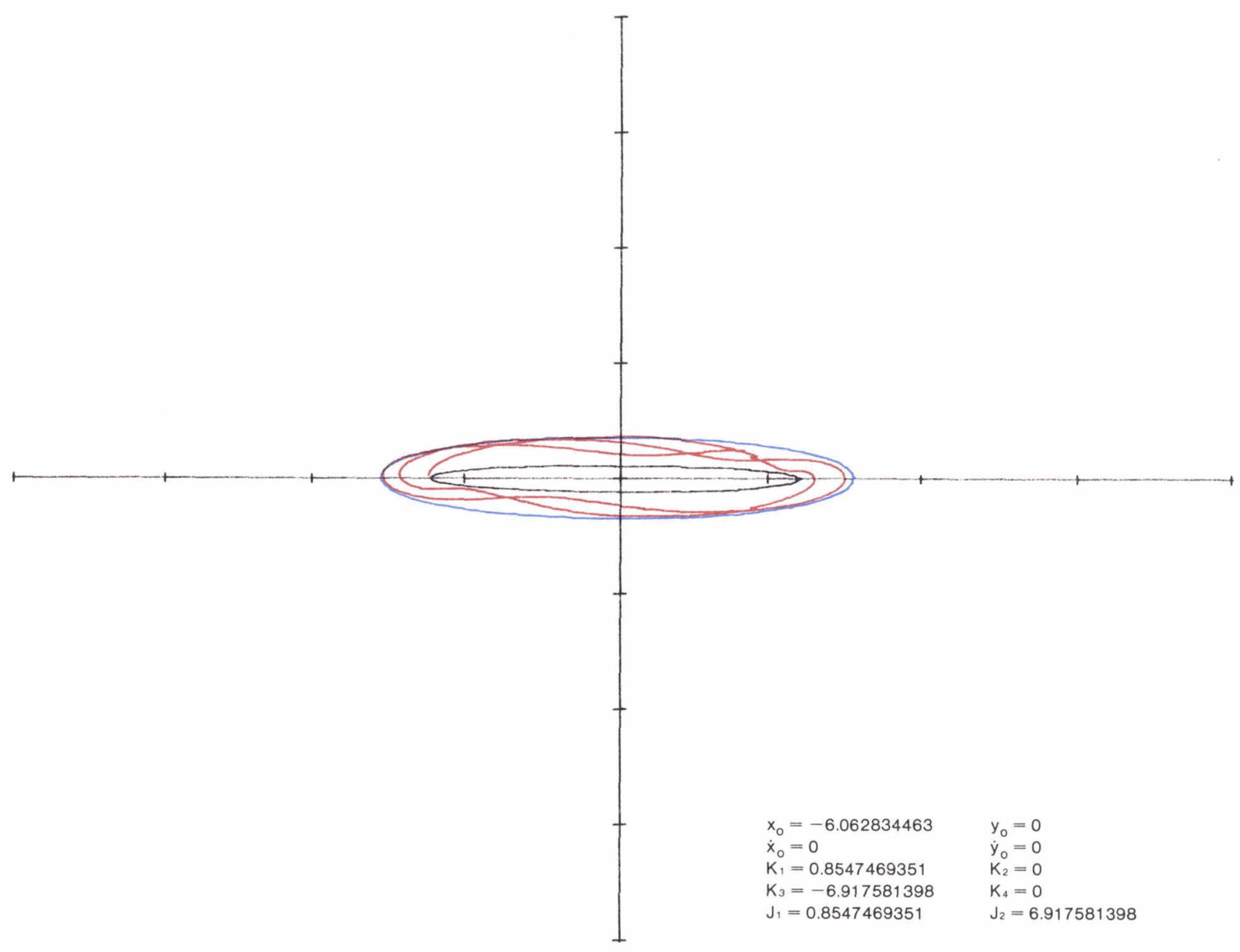

(m) $\alpha=0, \beta=0, \gamma=180^{\circ}$.

FIGURE 1.-Continued. 


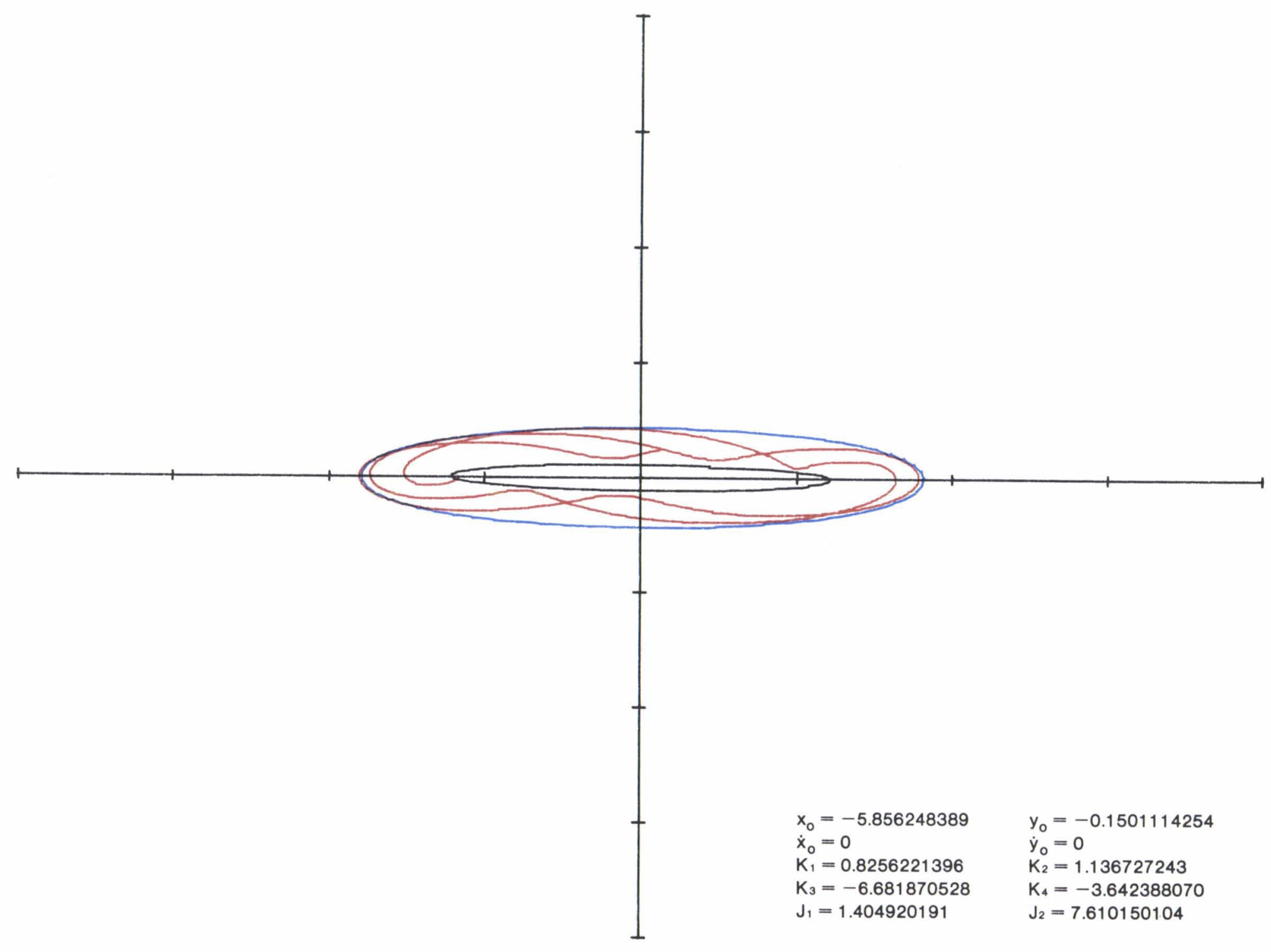

(n) $\alpha=0, \beta=0, \gamma=195^{\circ}$.

FIGURE 1.-Continued. 


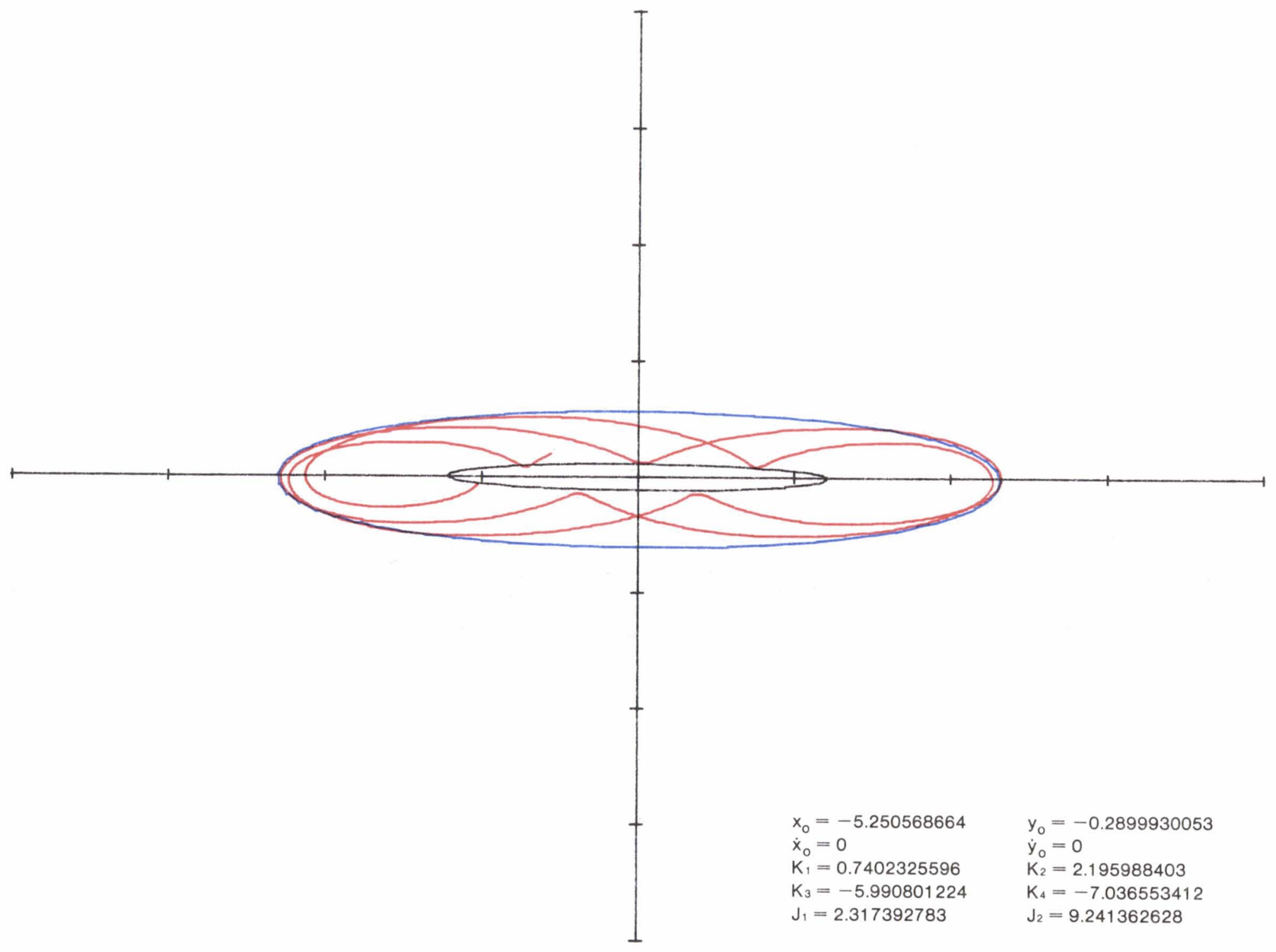

(o) $\alpha=0, \beta=0, \gamma=210^{\circ}$.

FIGURE 1.-Continued. 


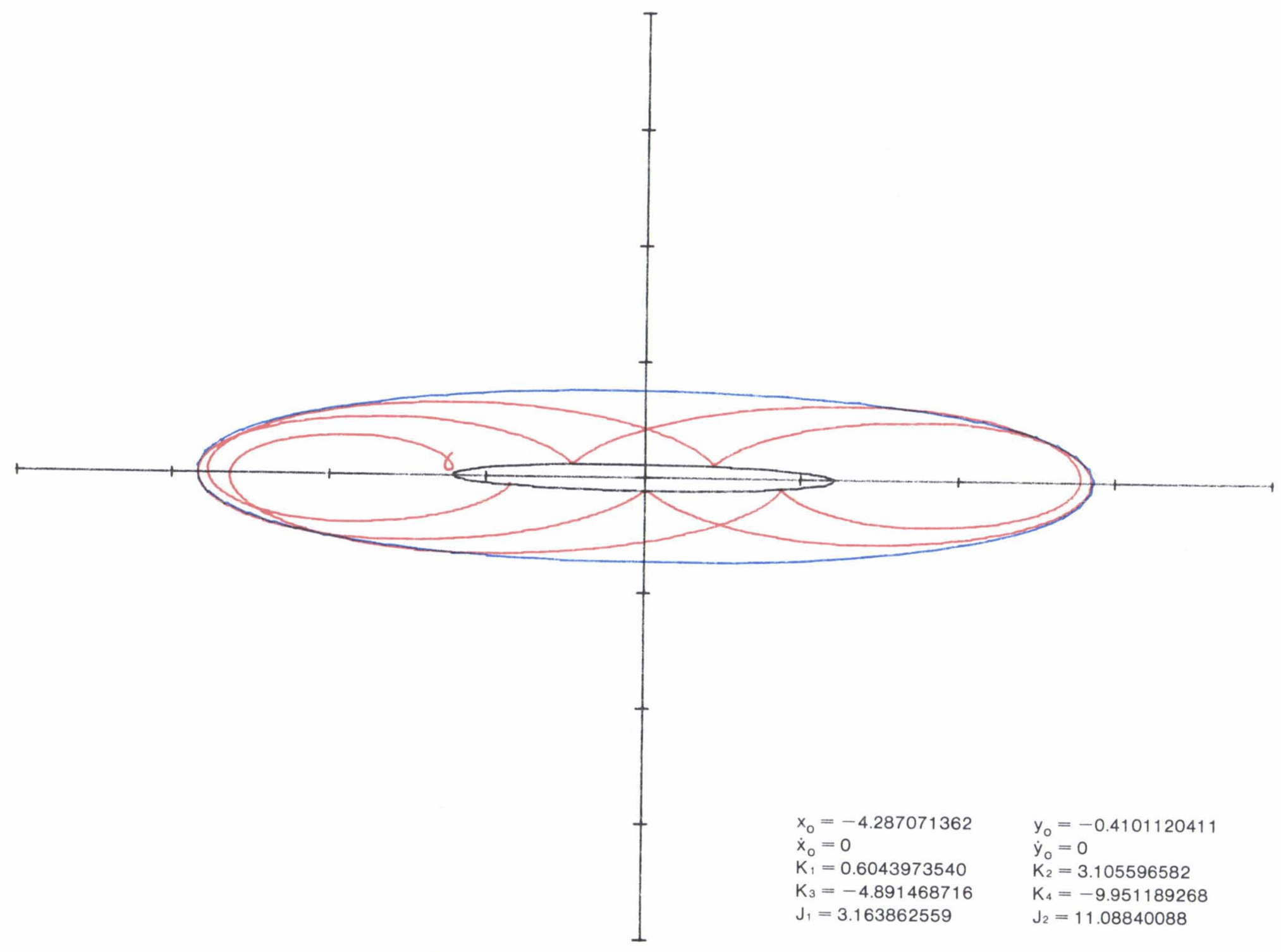

(p) $\alpha=0, \beta=0, \gamma=225^{\circ}$.

FIGURE 1.-Continued. 


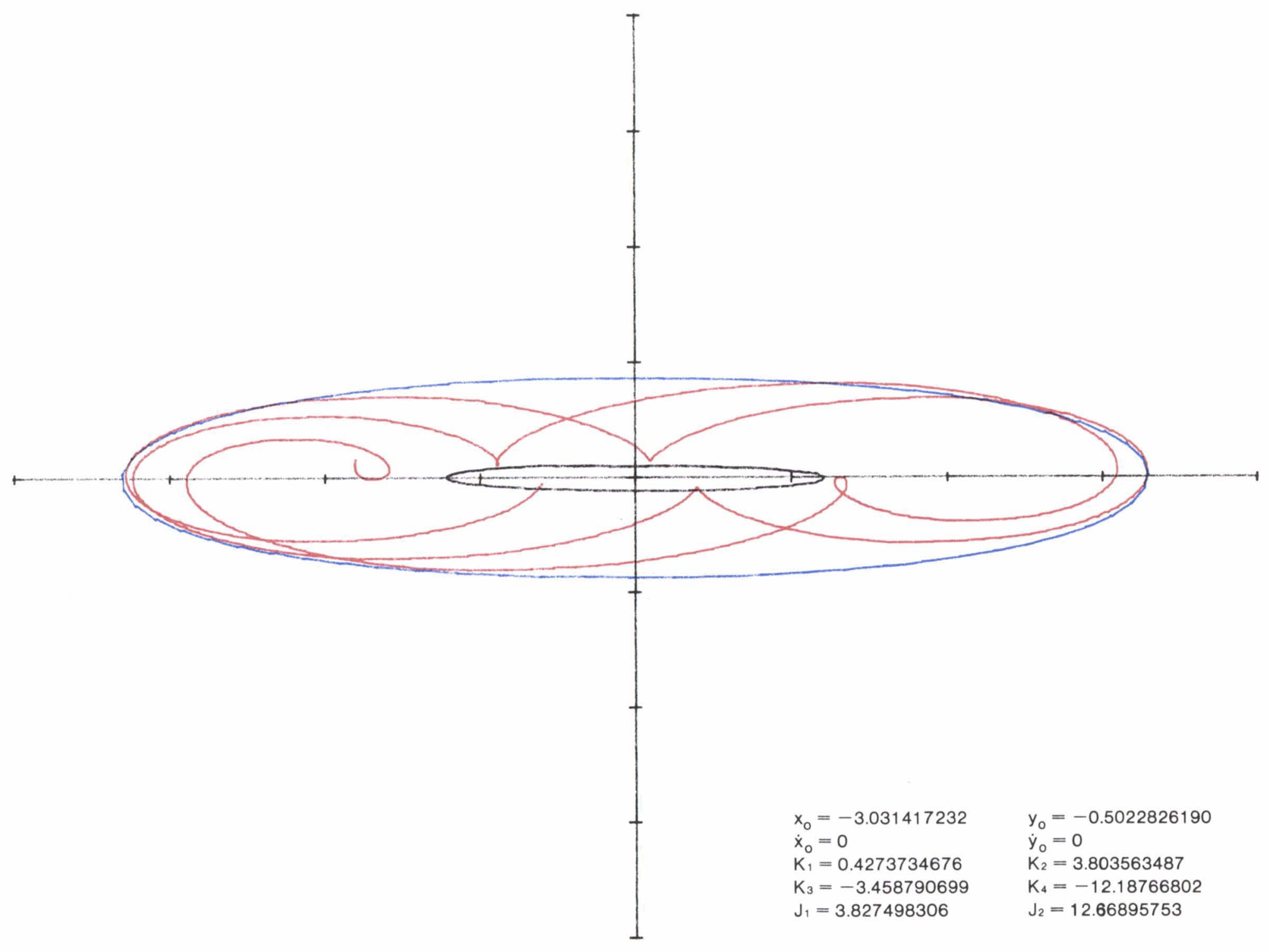

(q) $\alpha=0, \beta=0, \gamma=240^{\circ}$.

FIGURE 1.-Continued. 


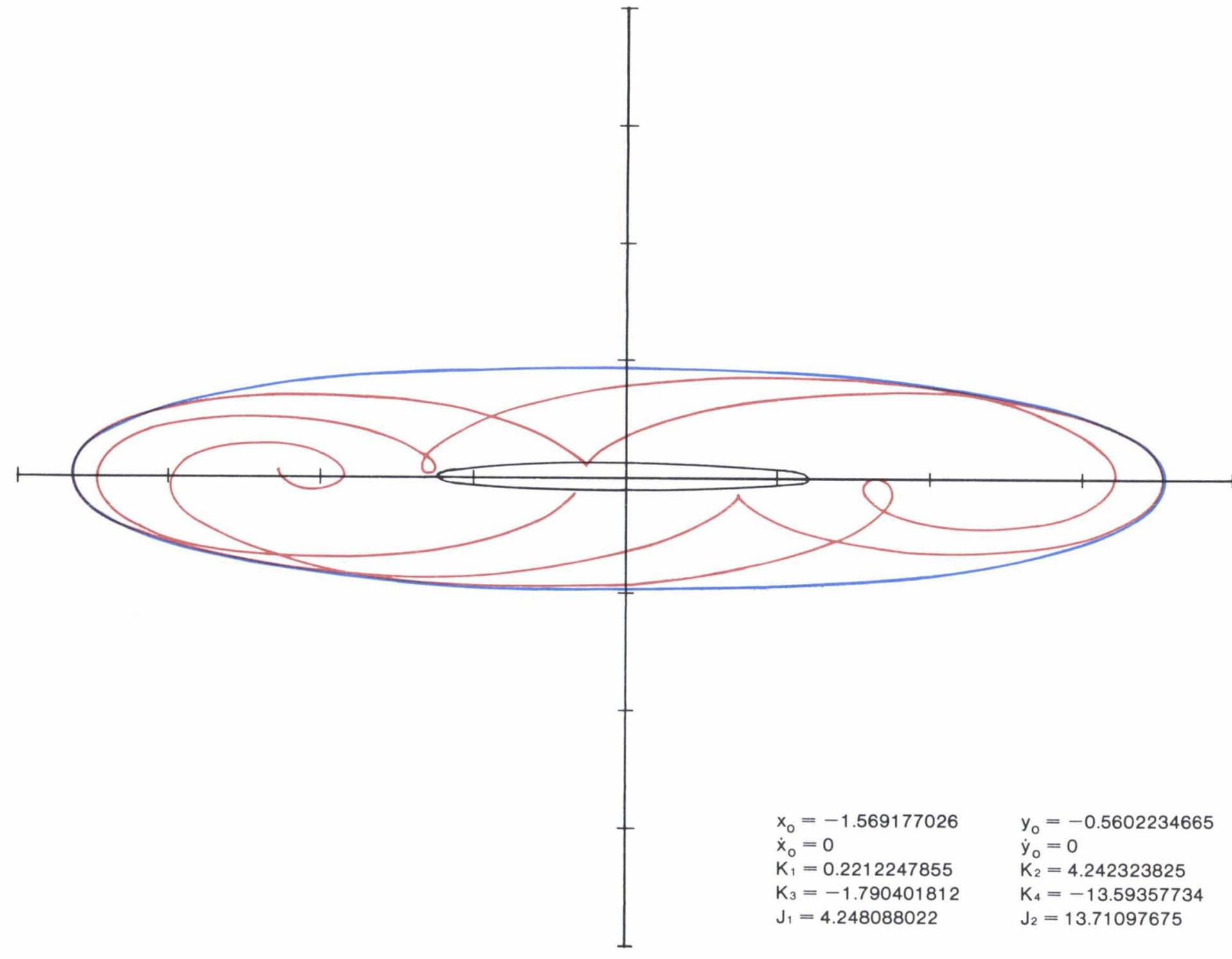

(r) $\alpha=0, \beta=0, \gamma=255^{\circ}$. 


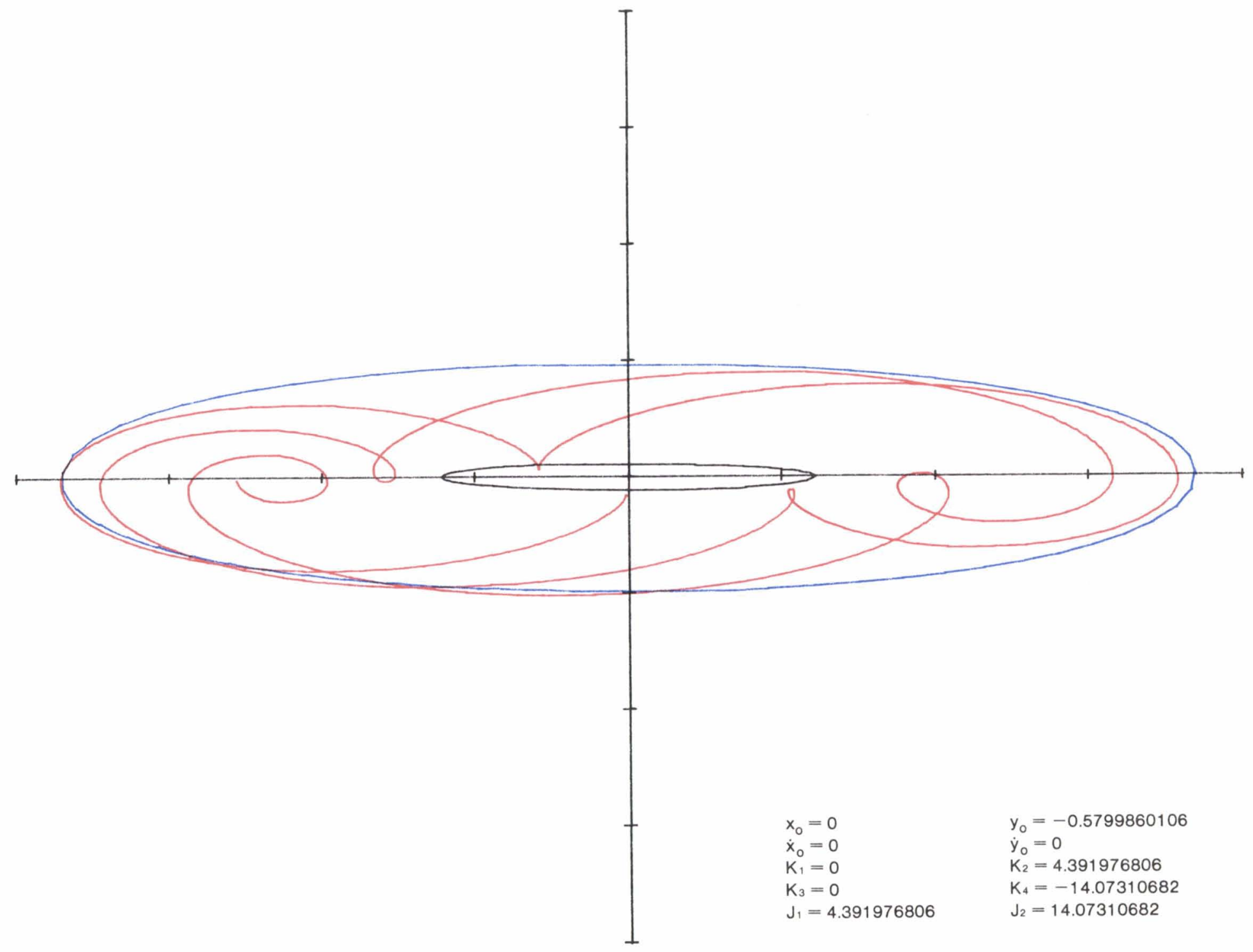

(s) $\alpha=\mathbf{0}, \beta=\mathbf{0}, \gamma=270^{\circ}$.

FIGURE 1.-Continued. 


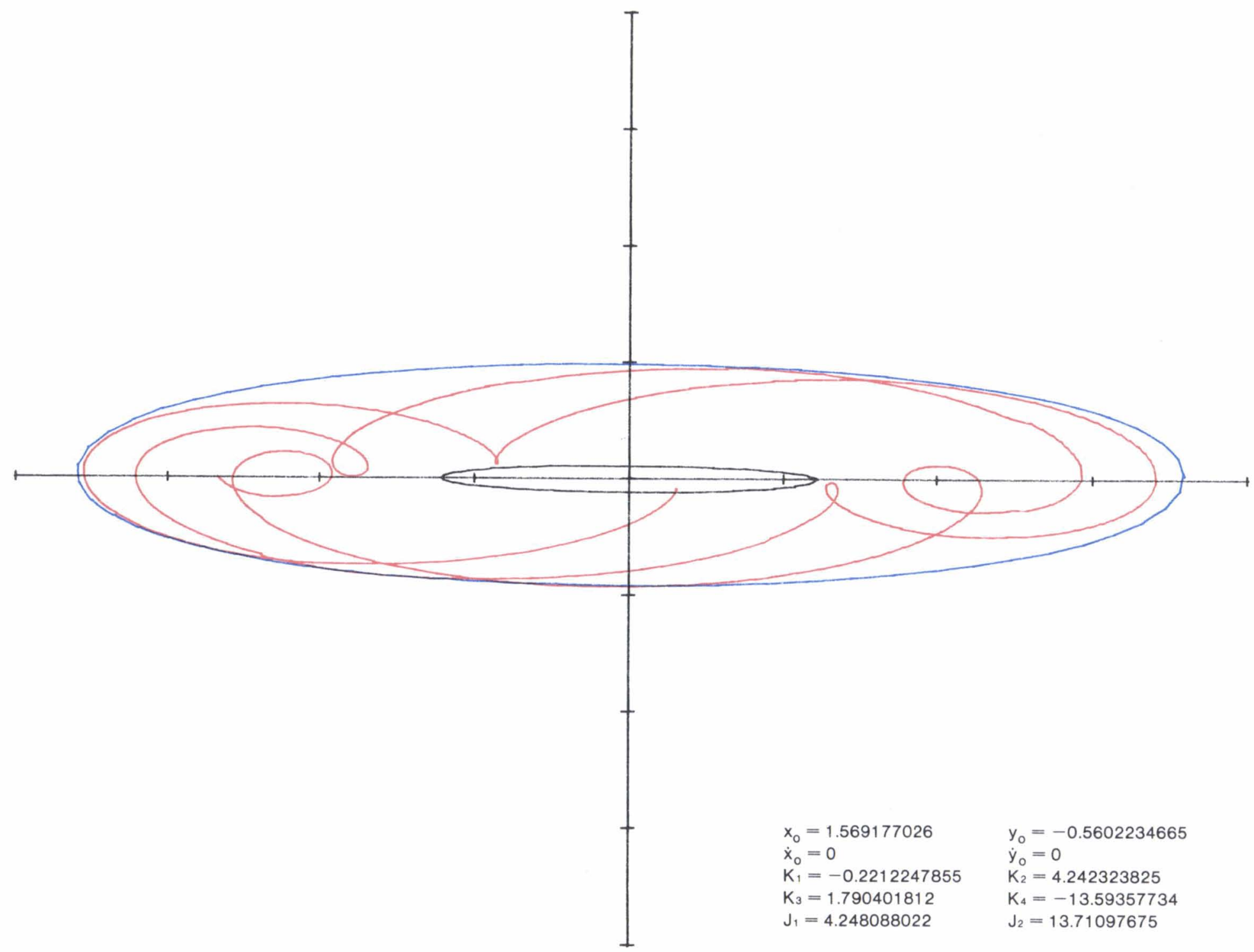

(t) $\alpha=0, \beta=0, \gamma=285^{\circ}$.

FIGURE 1.-Continued. 


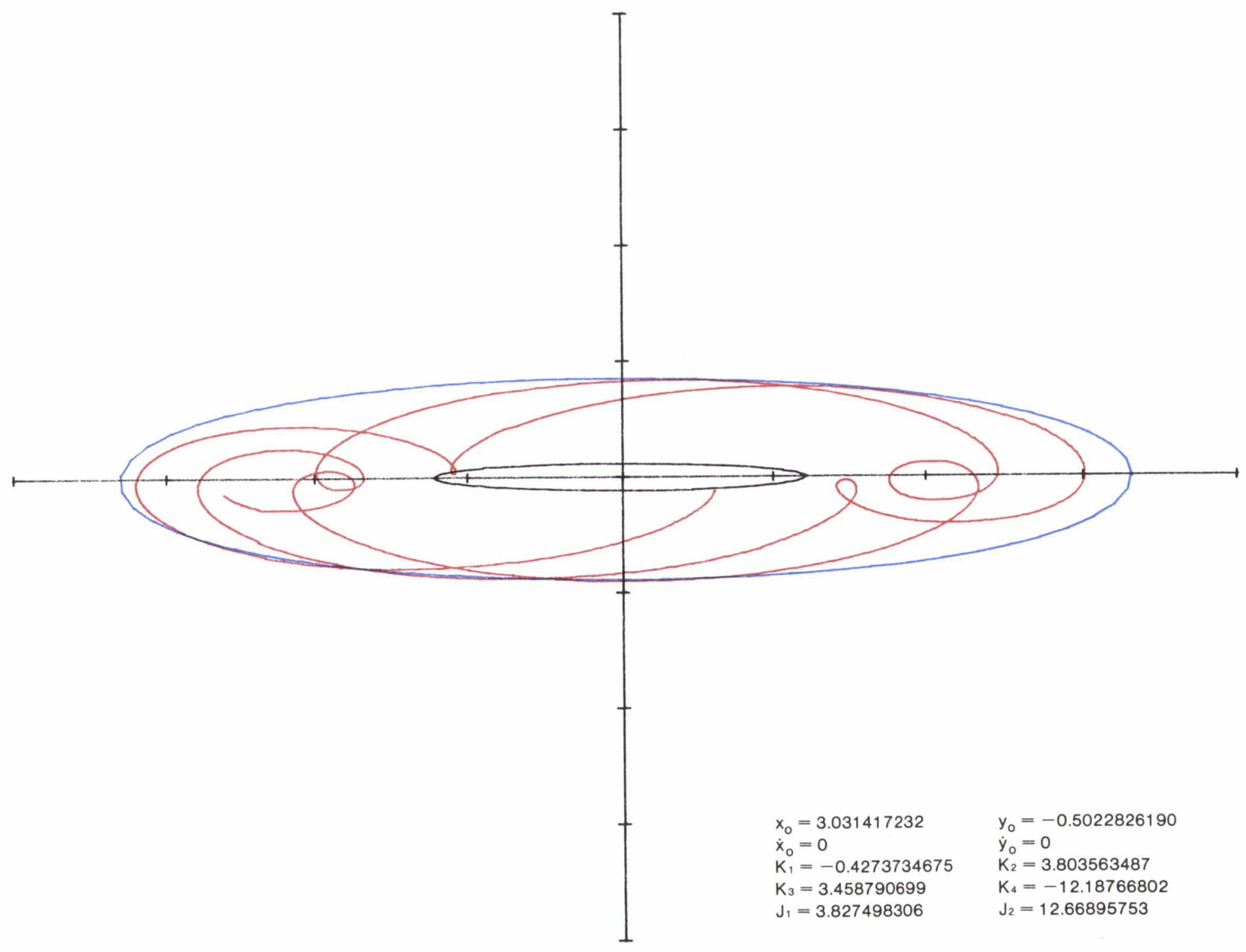

(u) $\alpha=\mathbf{0}, \beta=\mathbf{0}, \gamma=\mathbf{3 0 0 ^ { \circ }}$.

FIGURE 1.-Continued. 


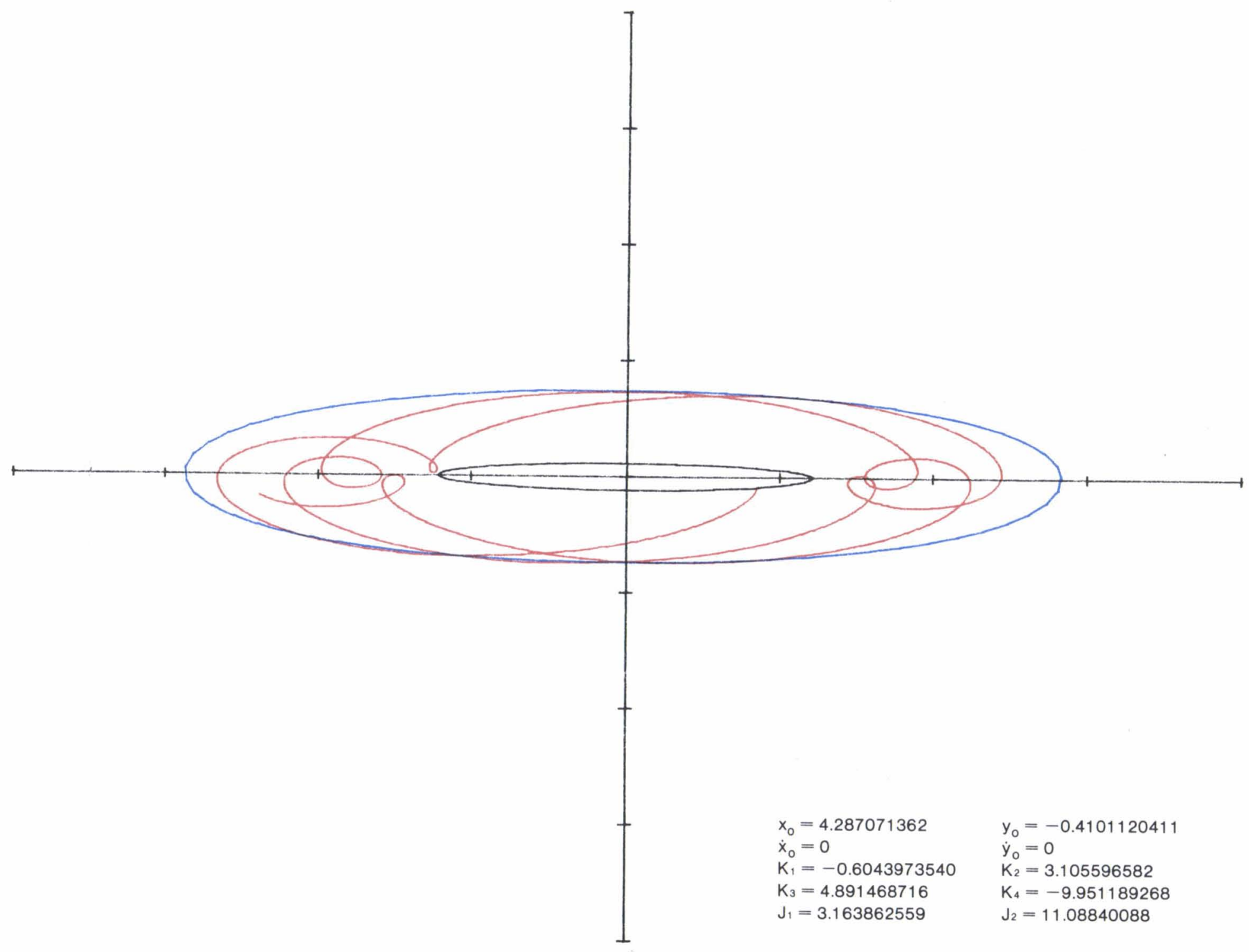

(v) $\alpha=0, \beta=0, \gamma=315^{\circ}$.

FIGURE 1.-Continued. 


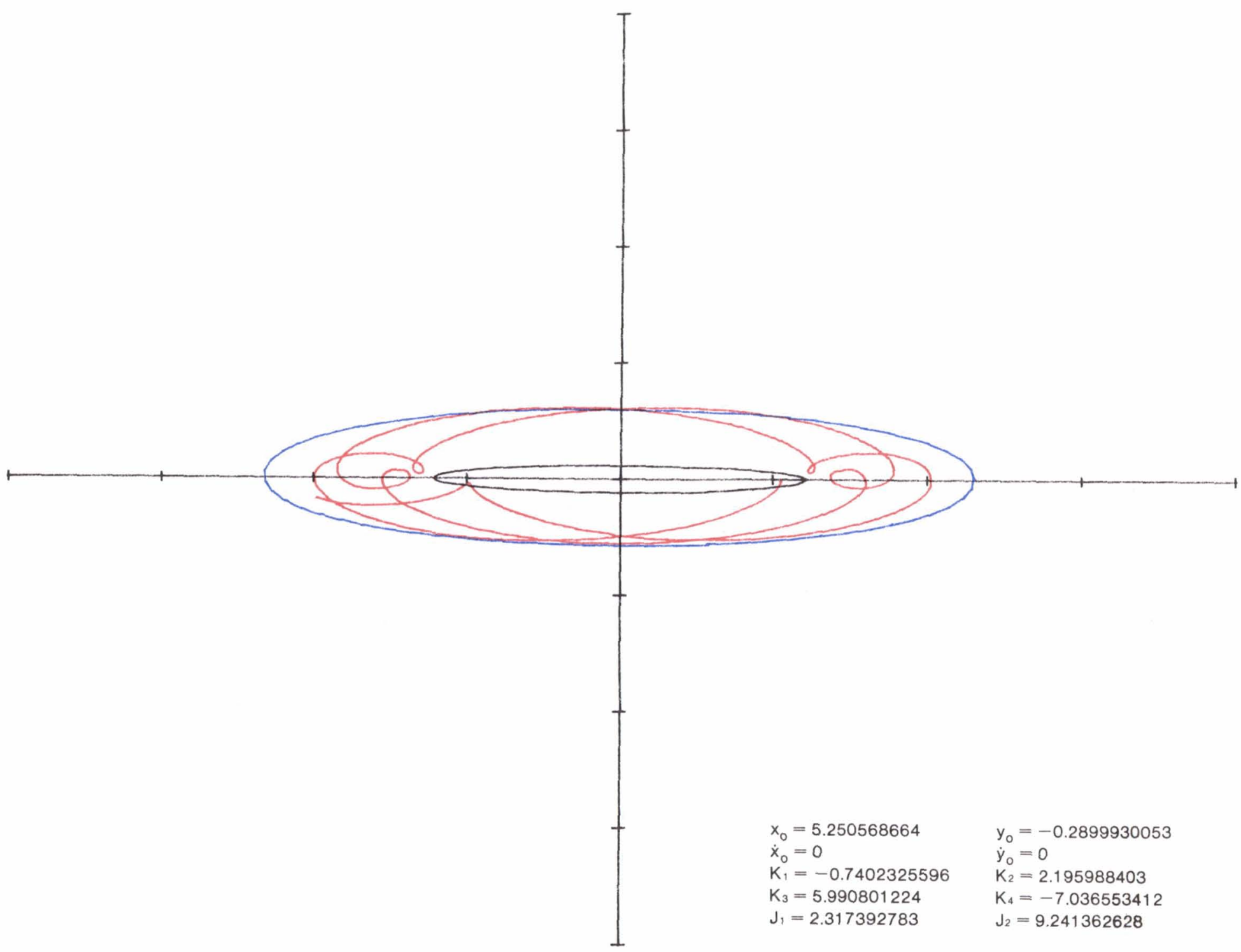

(w) $\alpha=\mathbf{0}, \beta=0, \gamma=330^{\circ}$.

FIGURE 1.-Continued. 


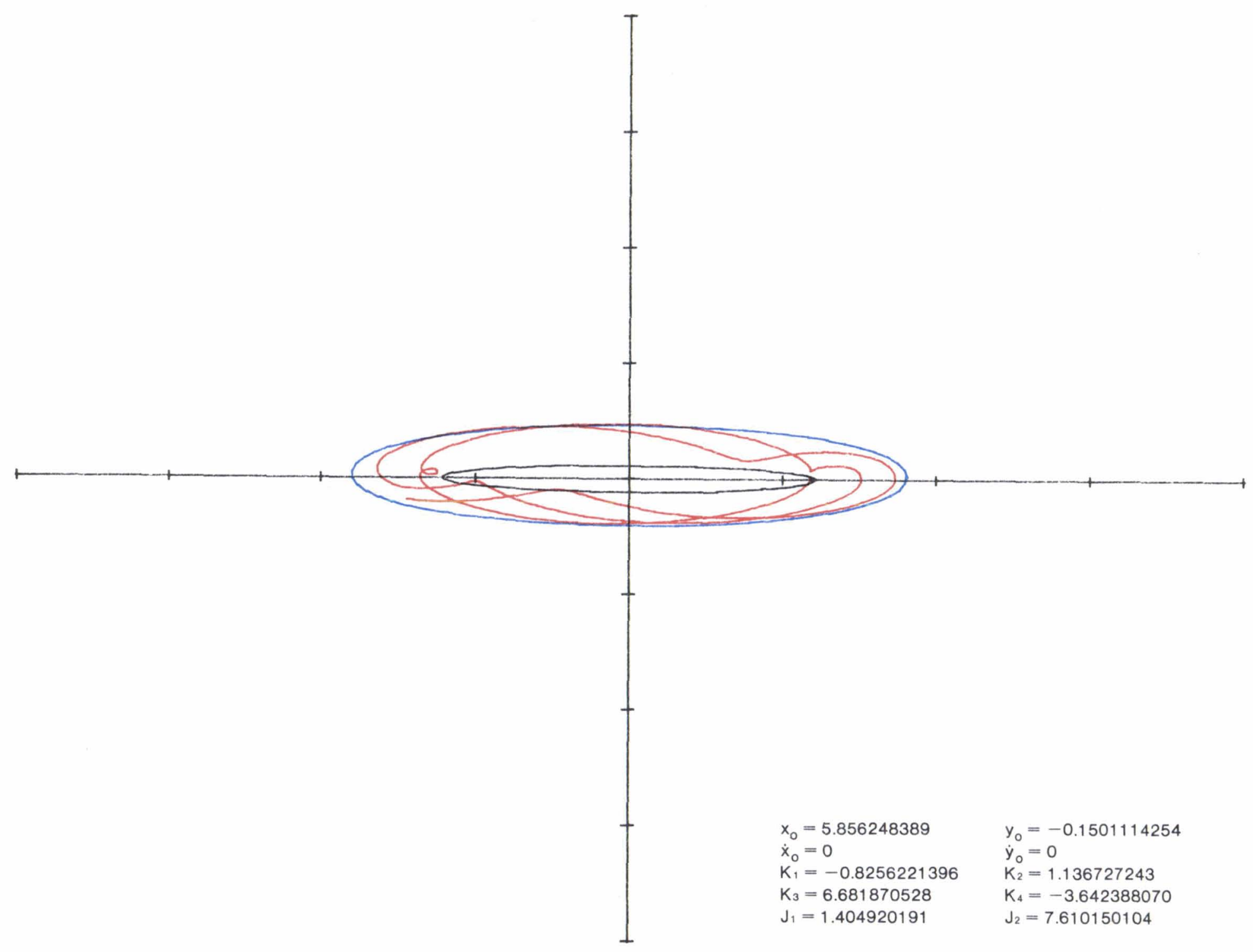

(x) $\alpha=0, \beta=0, \gamma=345^{\circ}$. 


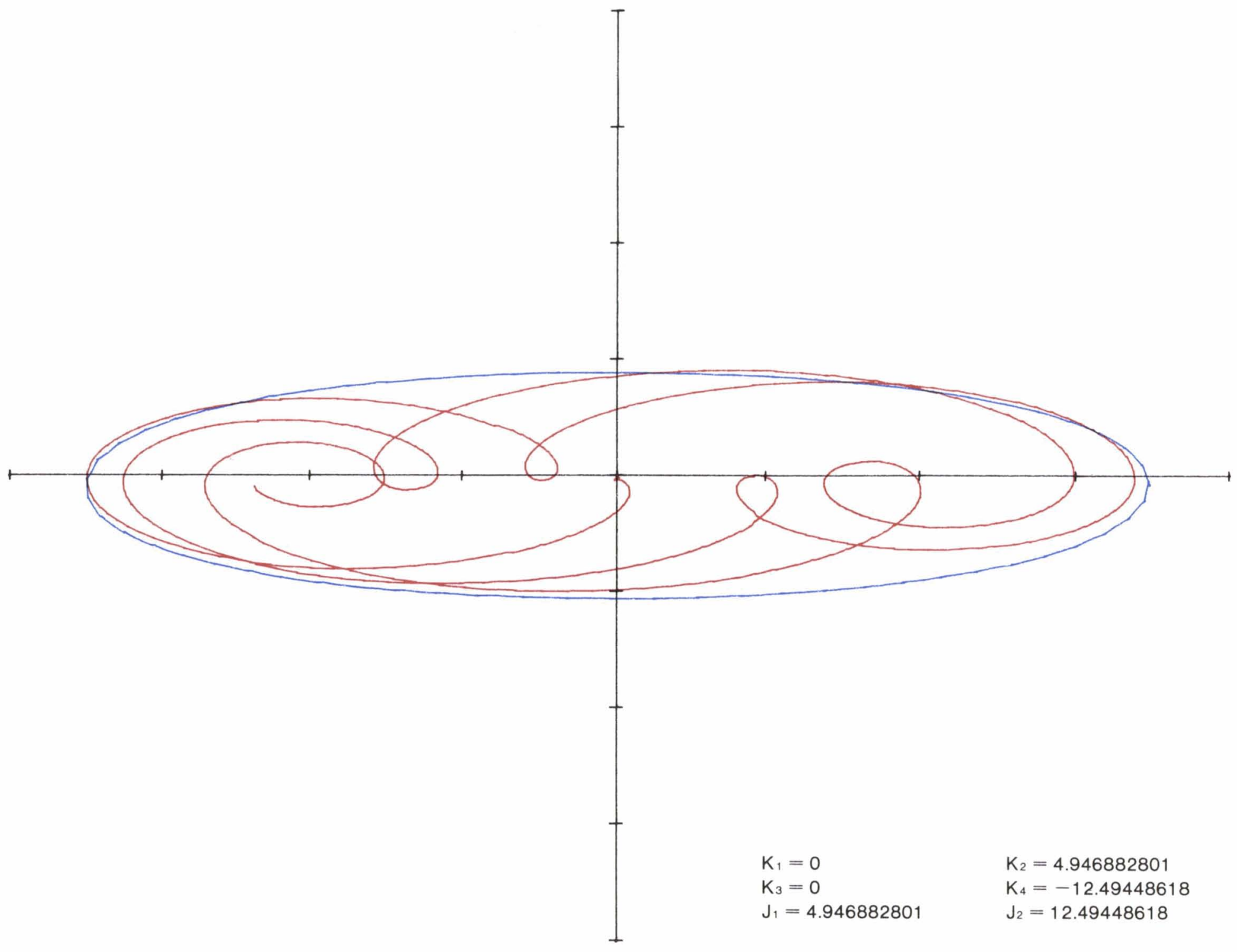

(a) $\alpha=0, \beta=0, \gamma=0^{\circ}$.

FIGURE 2.-Typical trajectories for which $C=+1$. 


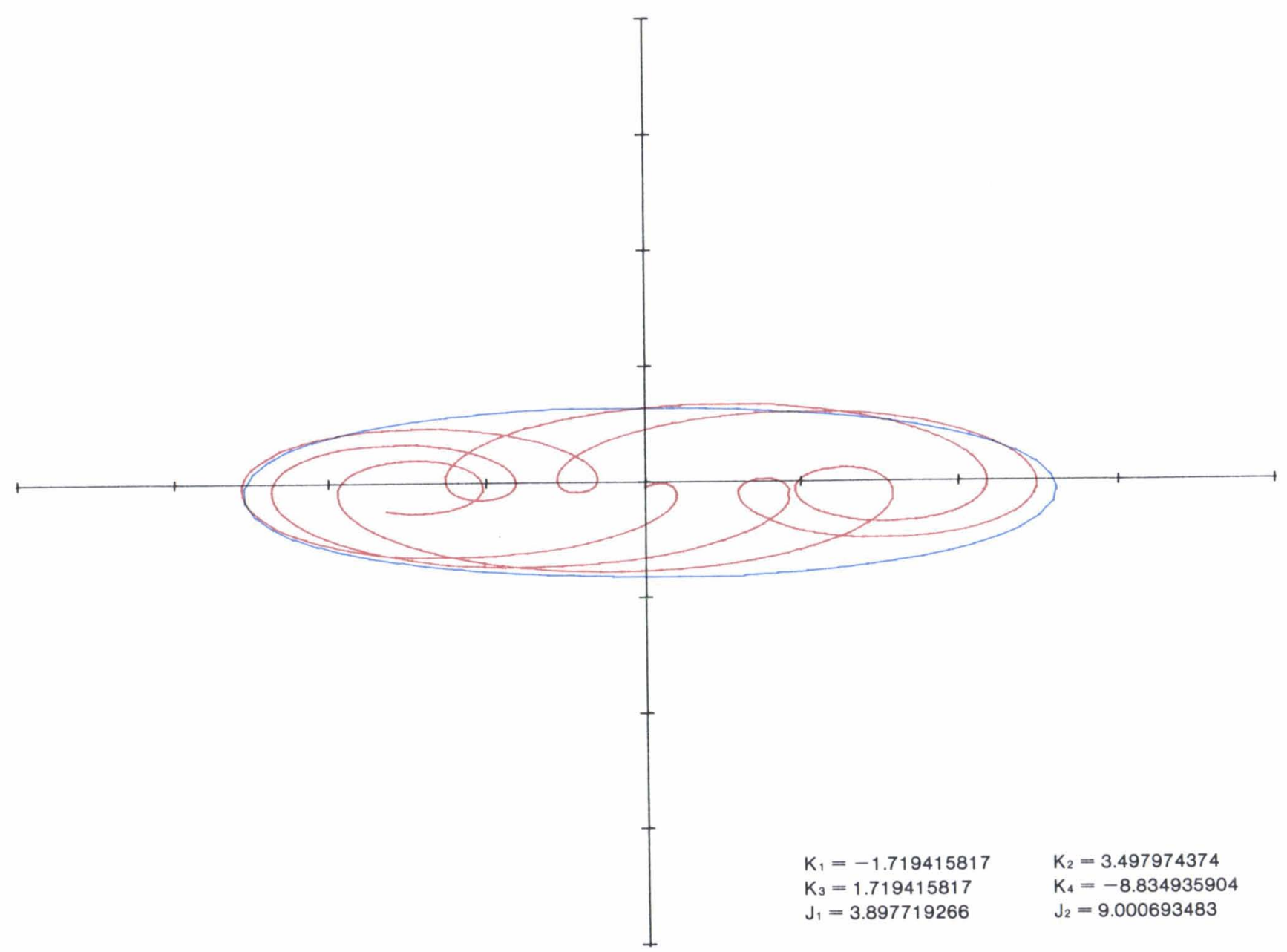

(b) $\alpha=0, \beta=0, \gamma=45^{\circ}$. 


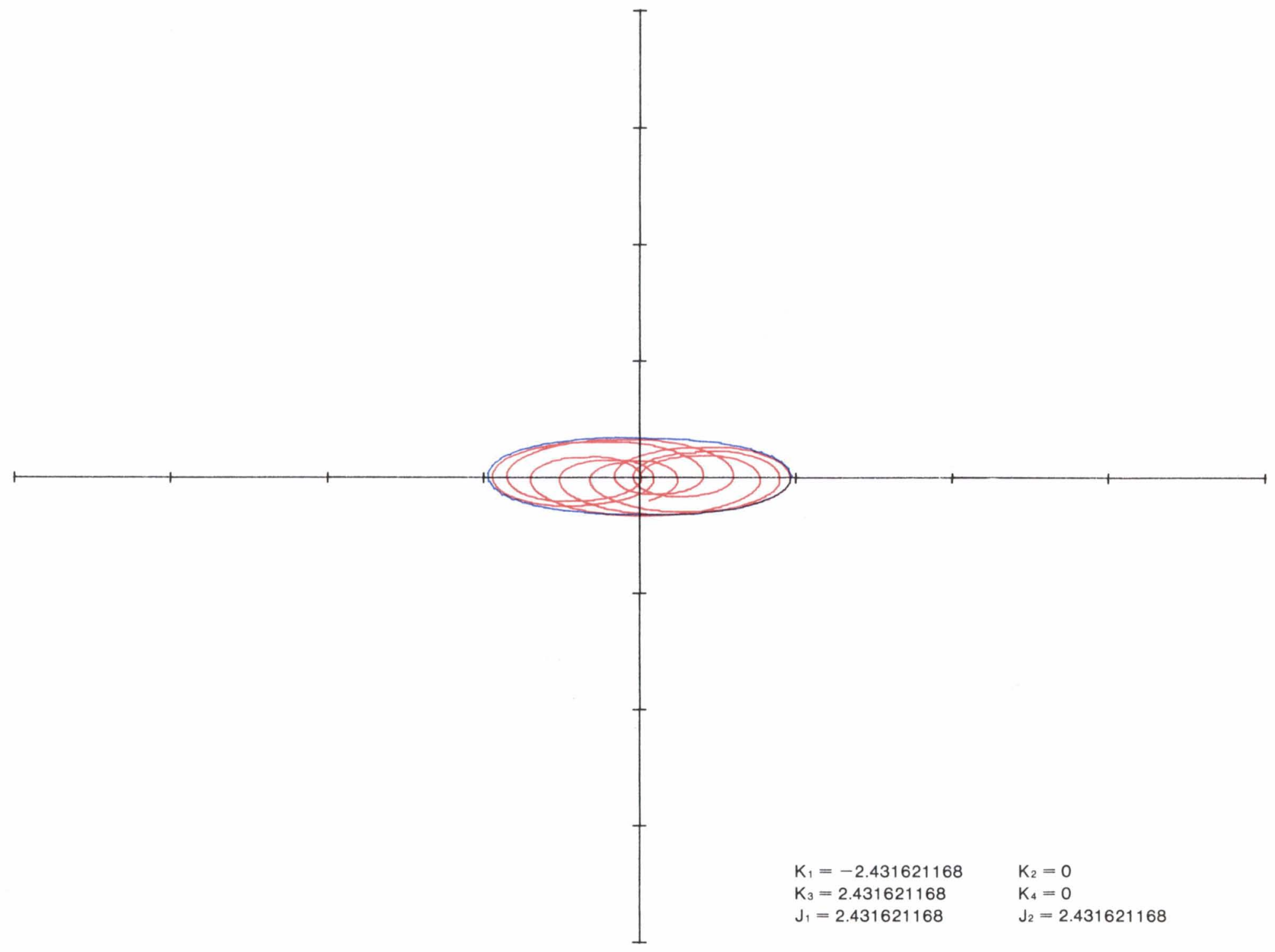

(c) $\alpha=0, \beta=0, \gamma=90^{\circ}$.

FIGURE 2.-Continued. 


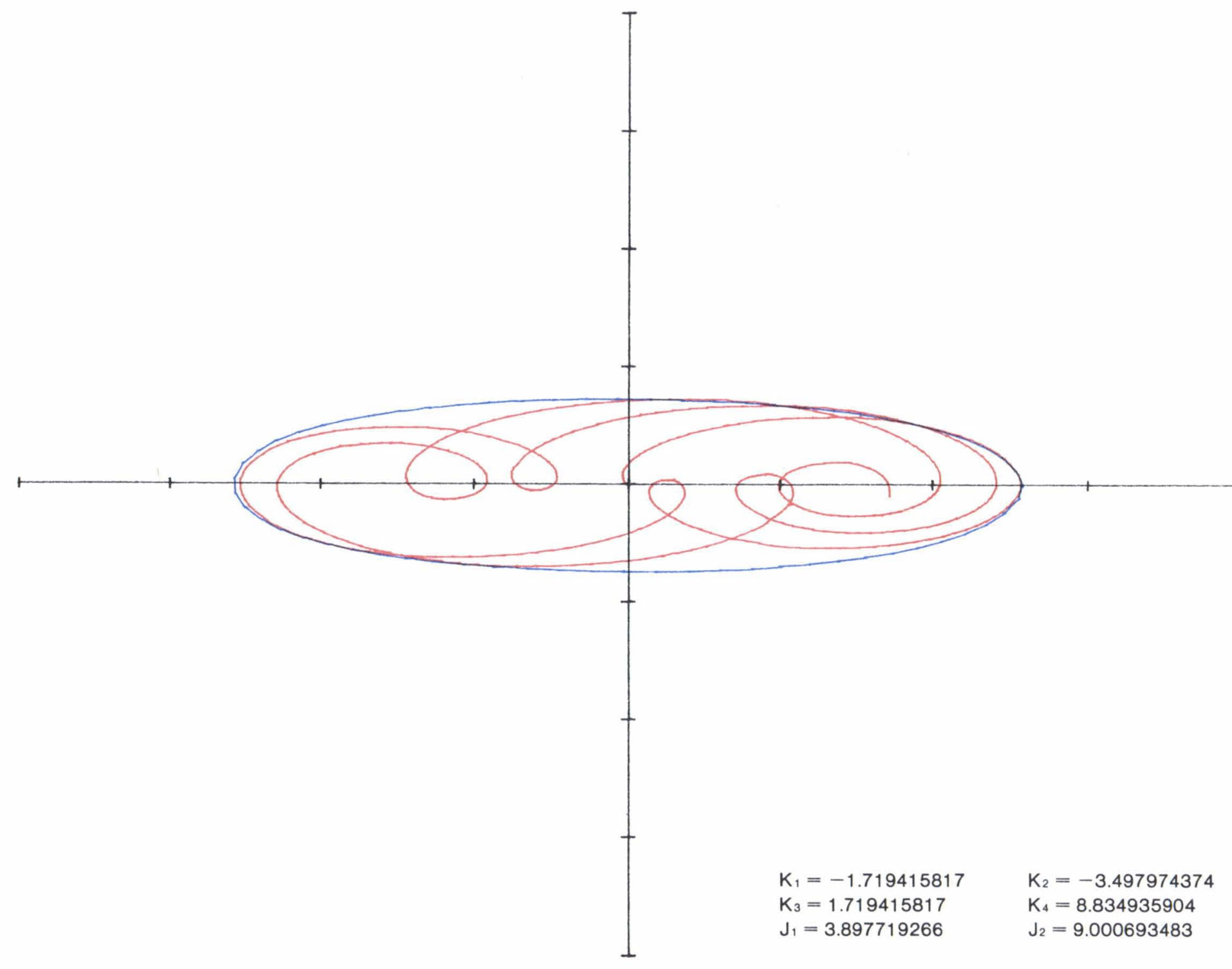

(d) $\alpha=0, \beta=0, \gamma=135^{\circ}$. 


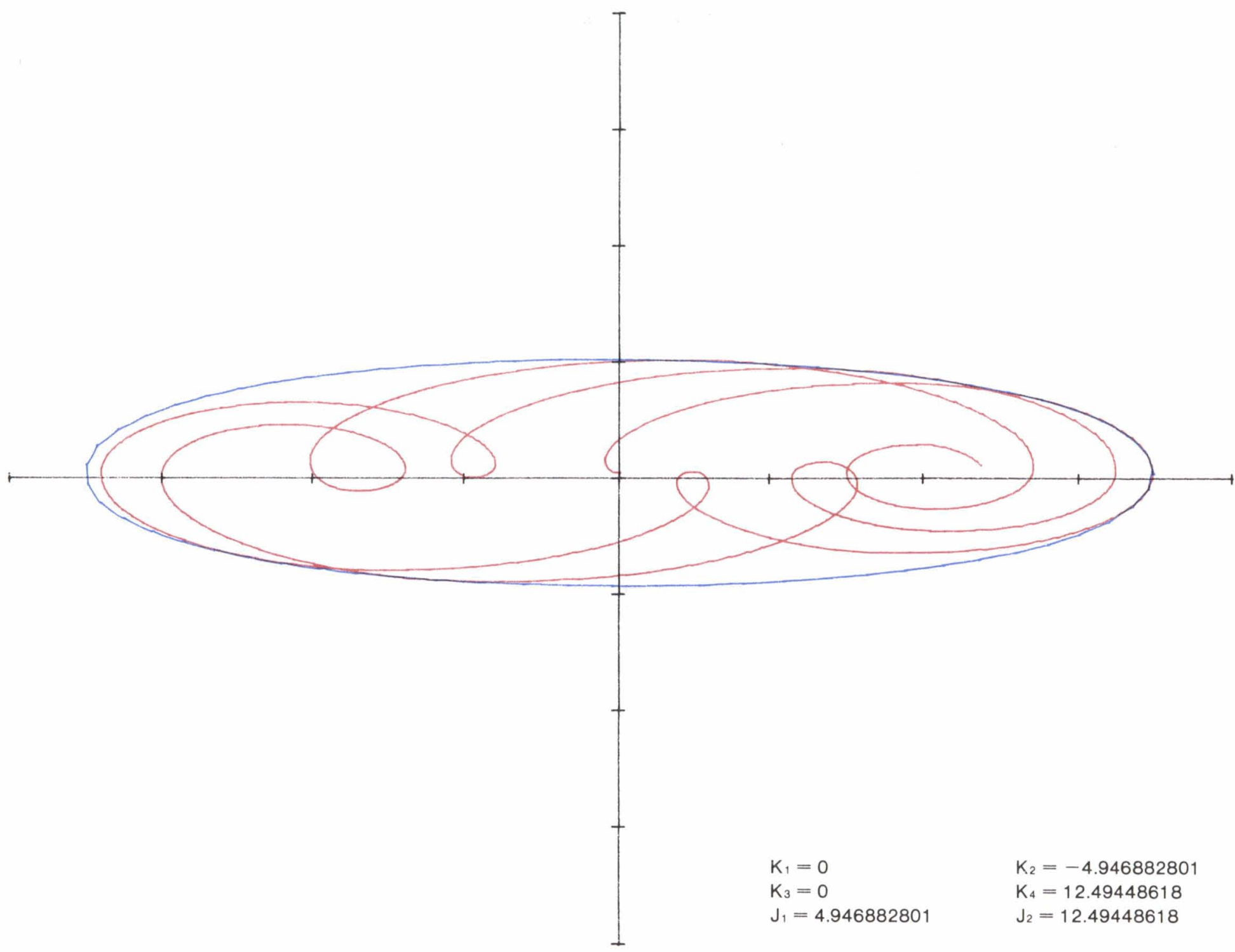

(e) $\alpha=0, \beta=0, \gamma=180^{\circ}$.

FIGURE 2.-Continued. 


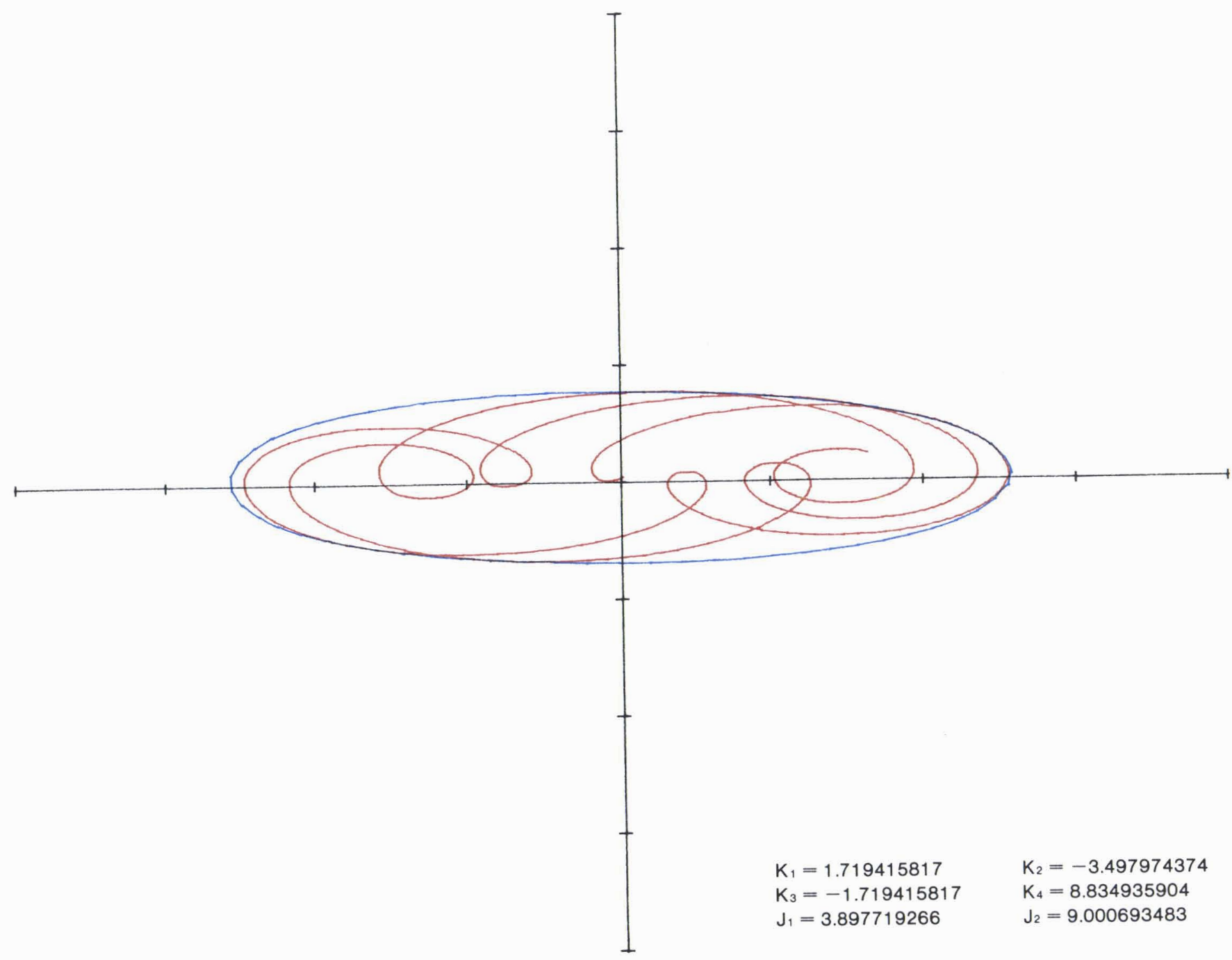

(f) $\alpha=0, \beta=0, \gamma=225^{\circ}$. 


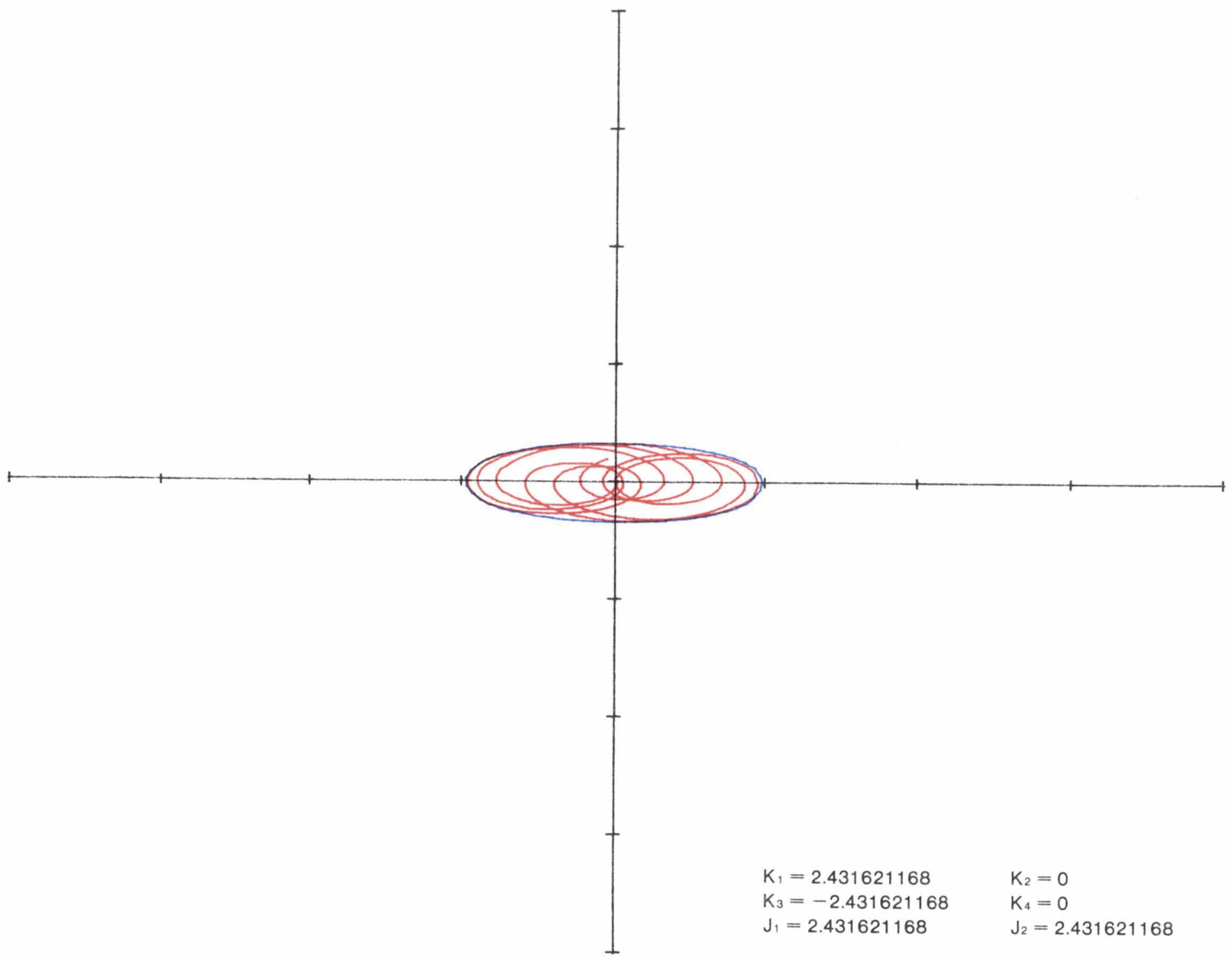

(g) $\alpha=0, \beta=0, \gamma=270^{\circ}$.

FIGURE 2.-Continued. 


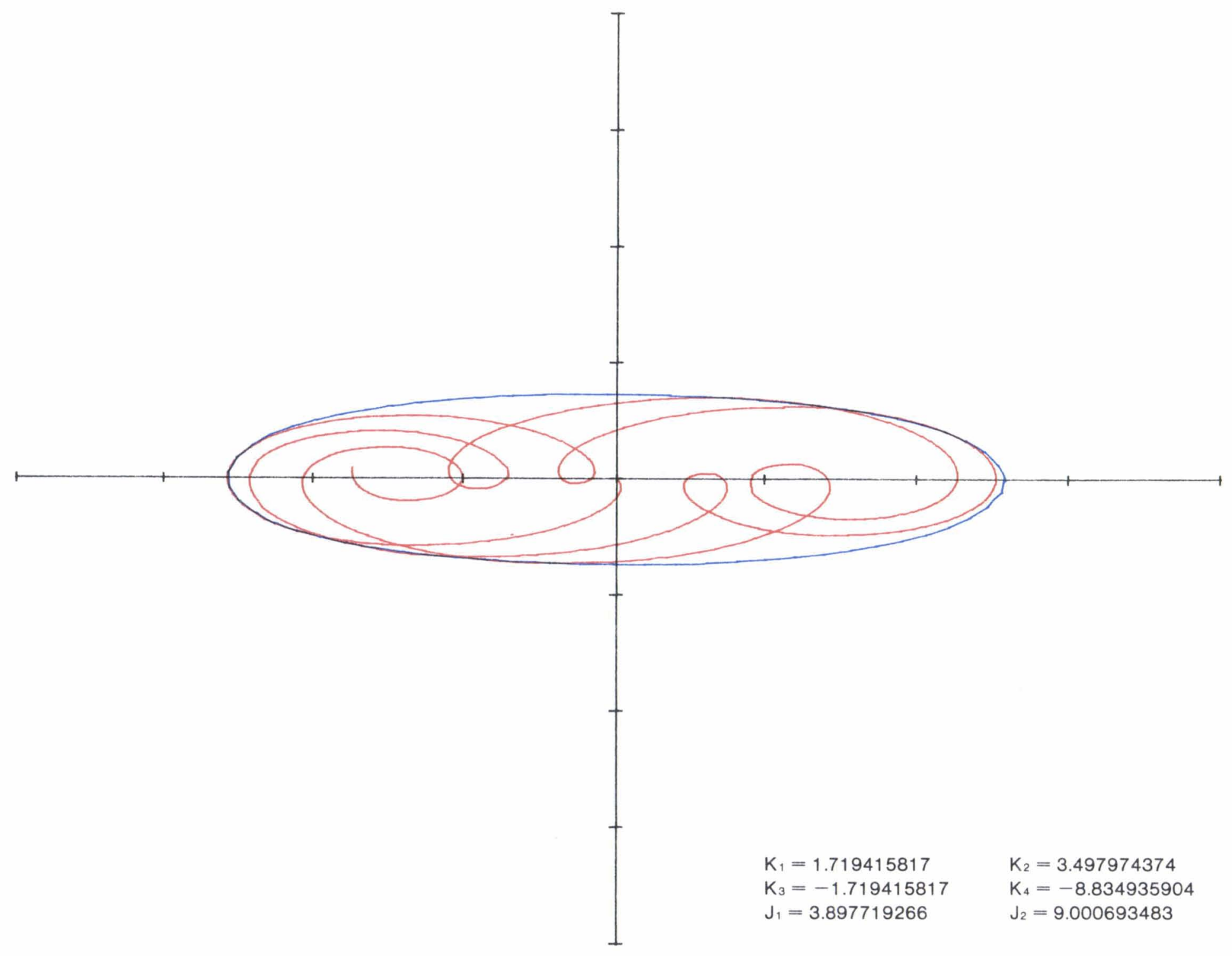

(h) $\alpha=0, \beta=0, \gamma=315^{\circ}$.

FIGURE 2.-Concluded. 


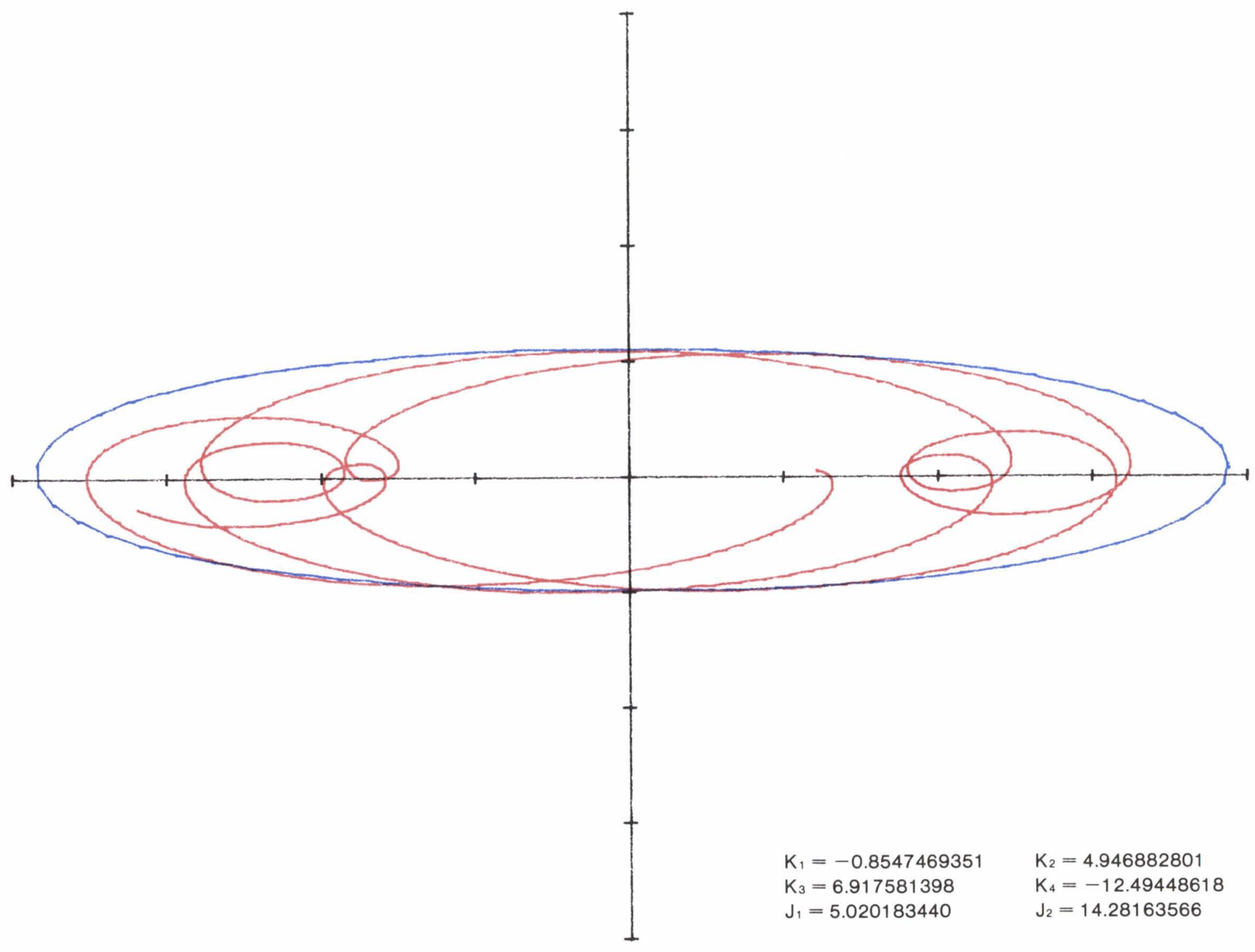

(a1) $\alpha=1, \beta=0^{\circ}, \gamma=0^{\circ}$.

FIGURE 3.-Typical trajectories for which $C=0$. 


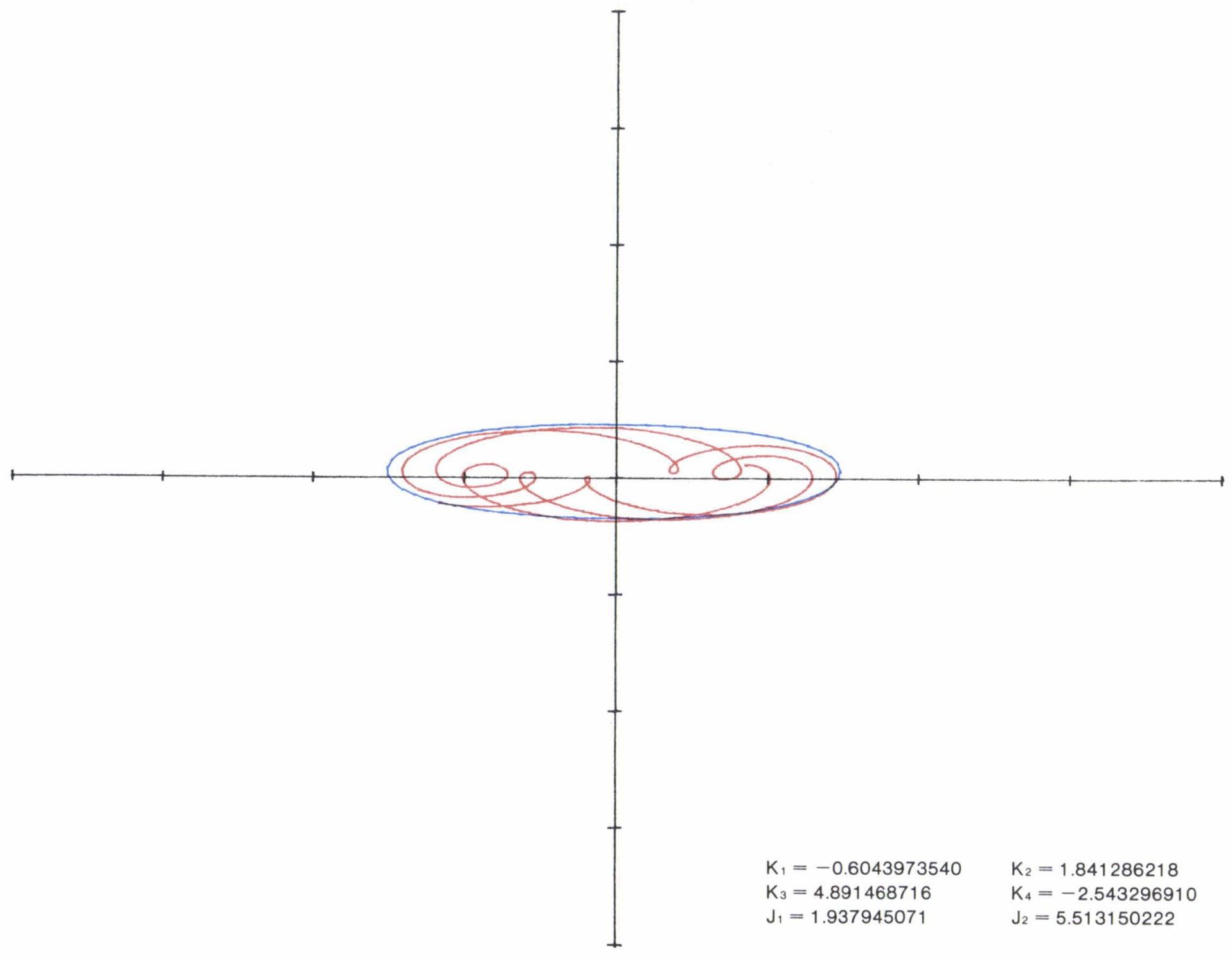

(a2) $\alpha=1, \beta=0^{\circ}, \gamma=45^{\circ}$. 


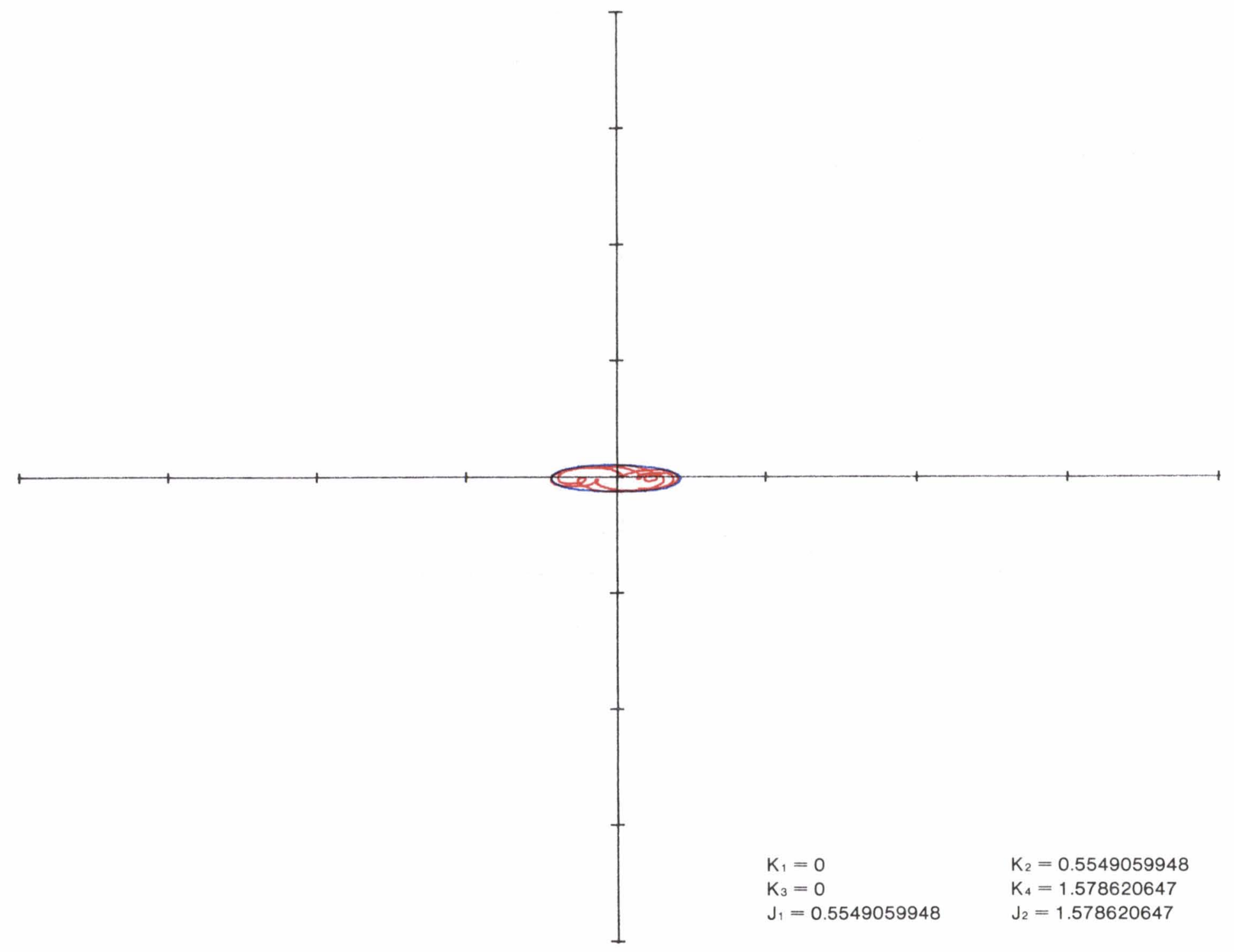

(a3) $\alpha=1, \beta=0^{\circ}, \gamma=90^{\circ}$.

FIGURE 3.-Continued. 


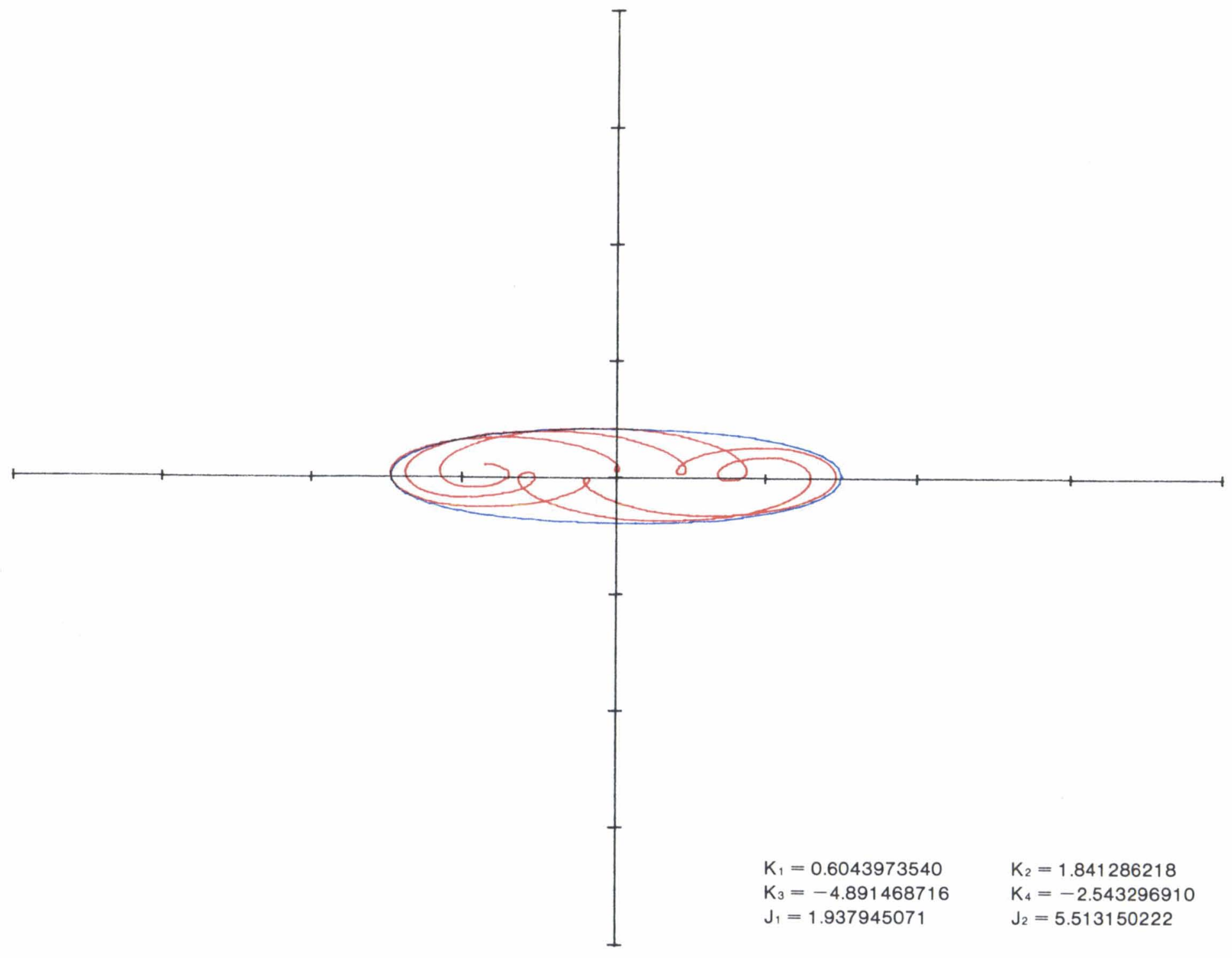

(a4) $\alpha=1, \beta=0^{\circ}, \gamma=135^{\circ}$.

FIGURE 3.-Continued. 


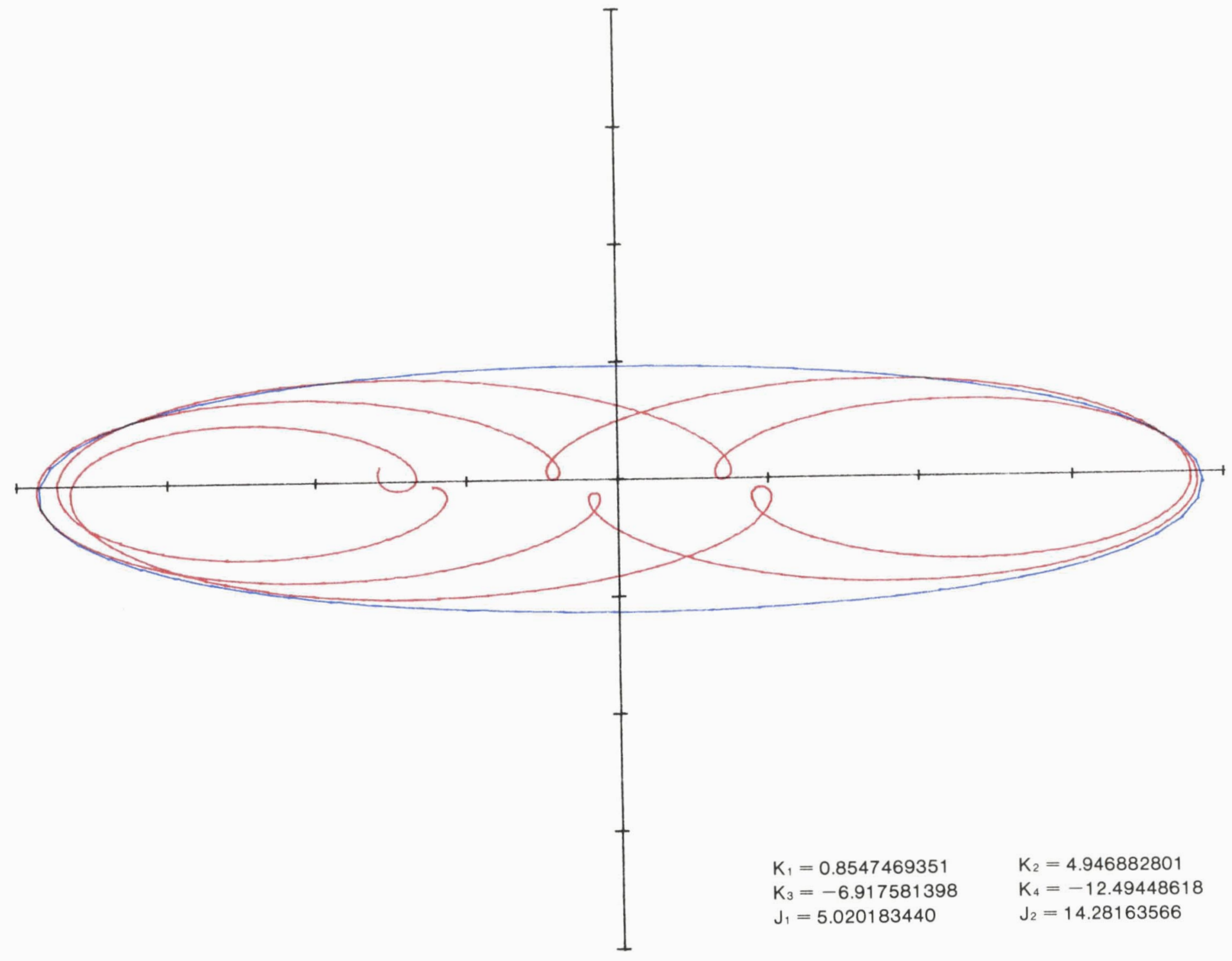

(a5) $\alpha=1, \beta=0^{\circ}, \gamma=180^{\circ}$.

FIGURE 3.-Continued. 


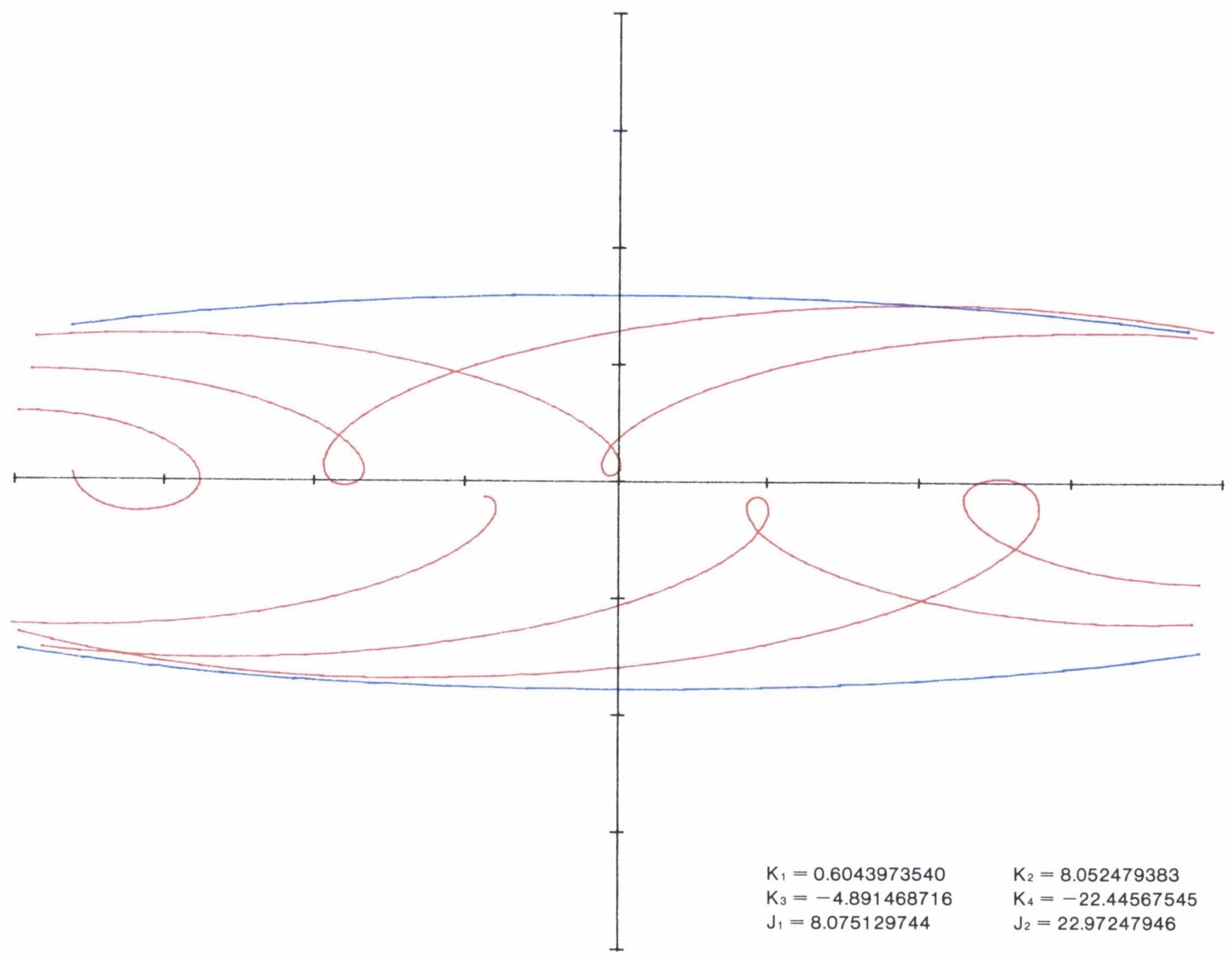

(a6) $\alpha=1, \beta=0^{\circ}, \gamma=225^{\circ}$.

un

FIGURE 3.-Continued. 


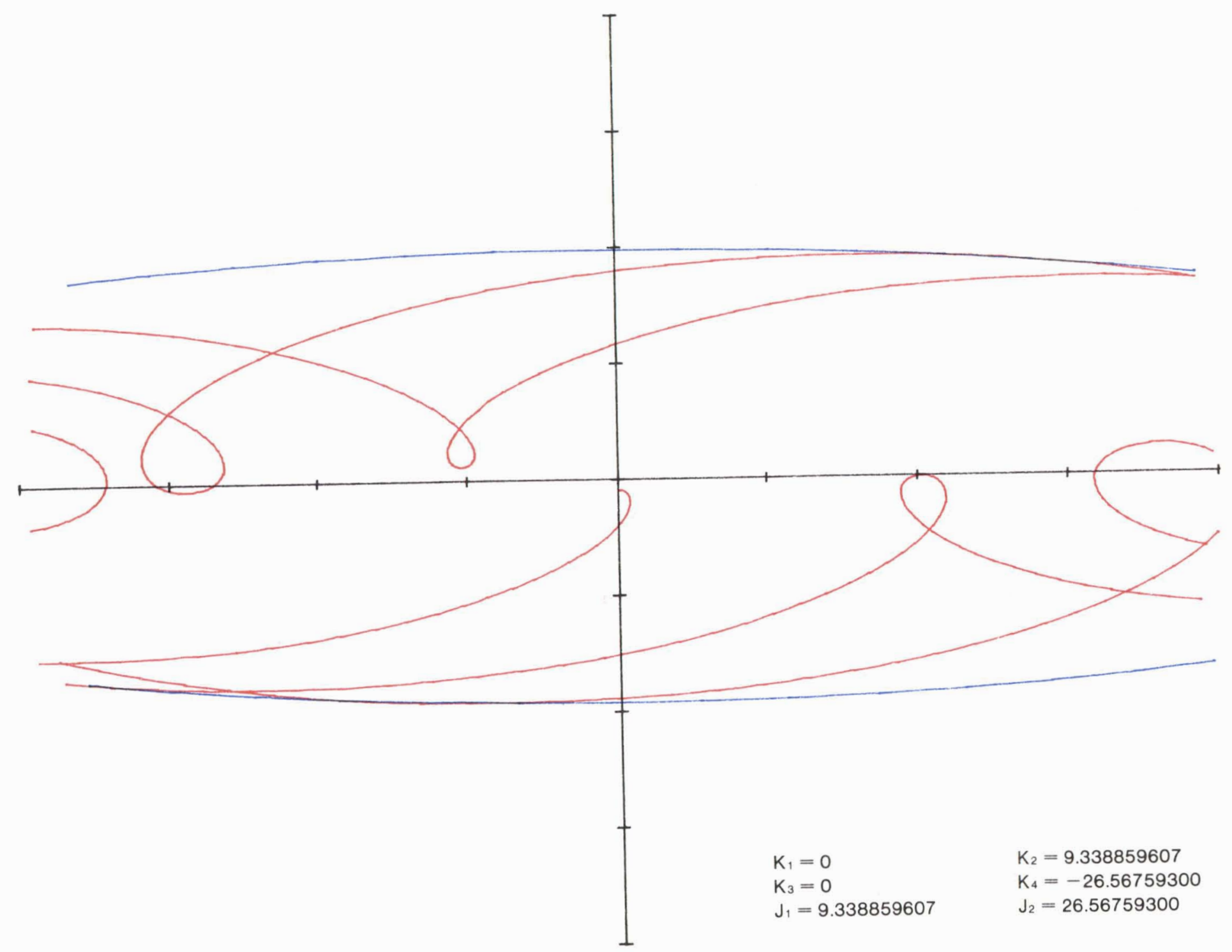

(a7) $\alpha=1, \beta=0^{\circ}, \gamma=270^{\circ}$.

FIGURE 3-Continued. 


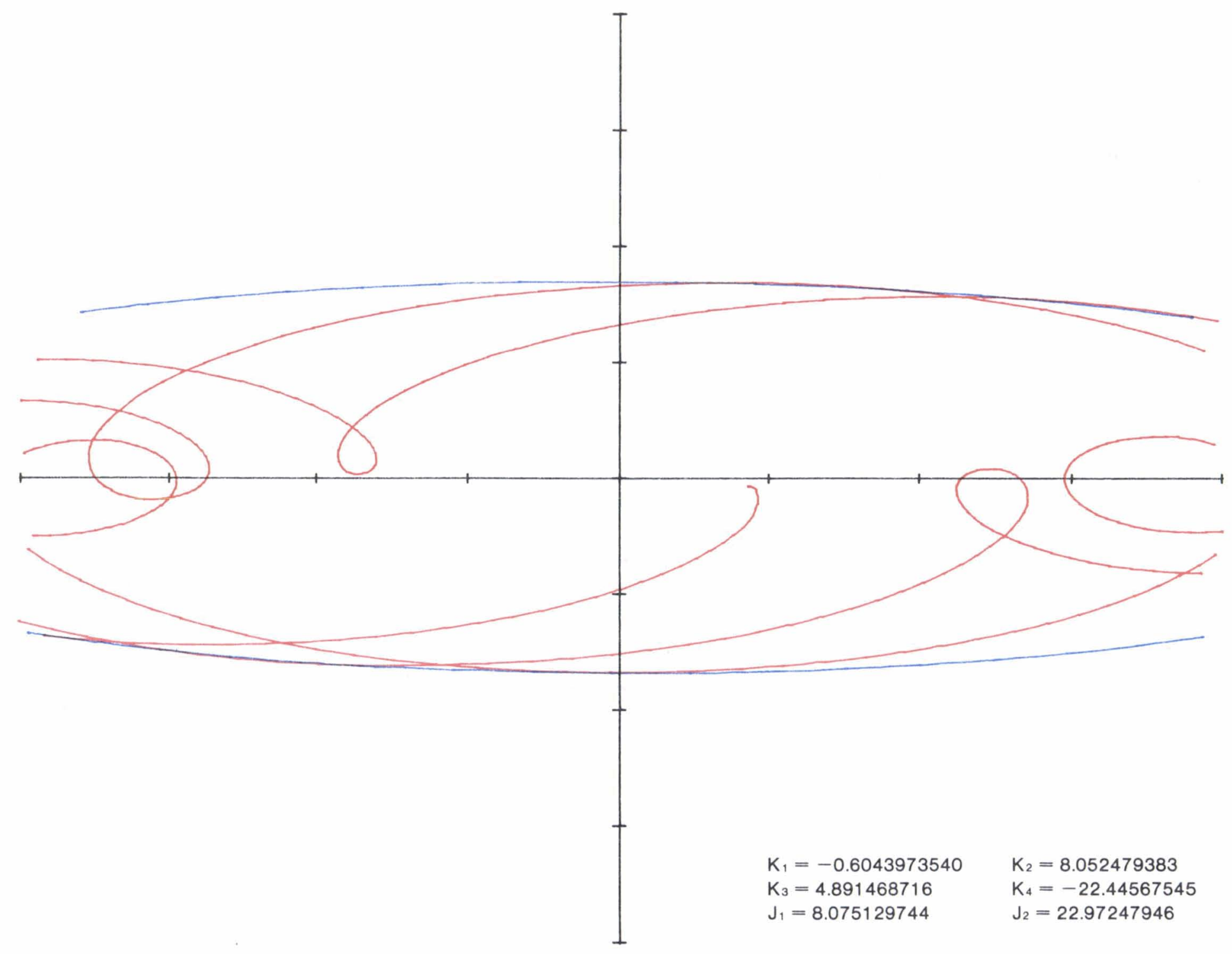

(a8) $\alpha=1, \beta=0^{\circ}, \gamma=315^{\circ}$

FIGURE 3.-Continued. 


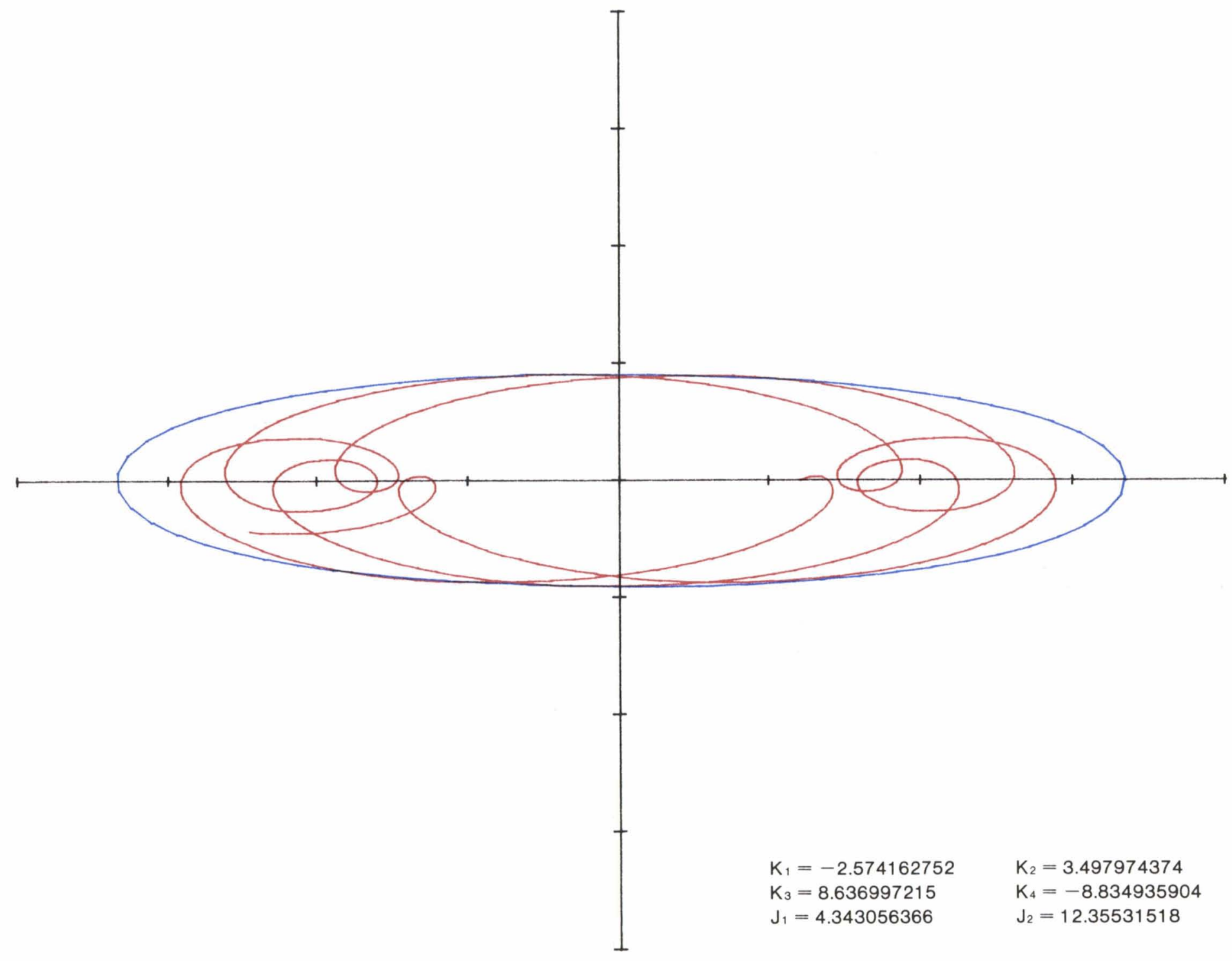

(b1) $\alpha=1, \beta=45^{\circ}, \gamma=0^{\circ}$.

FIGURE 3.-Continued. 


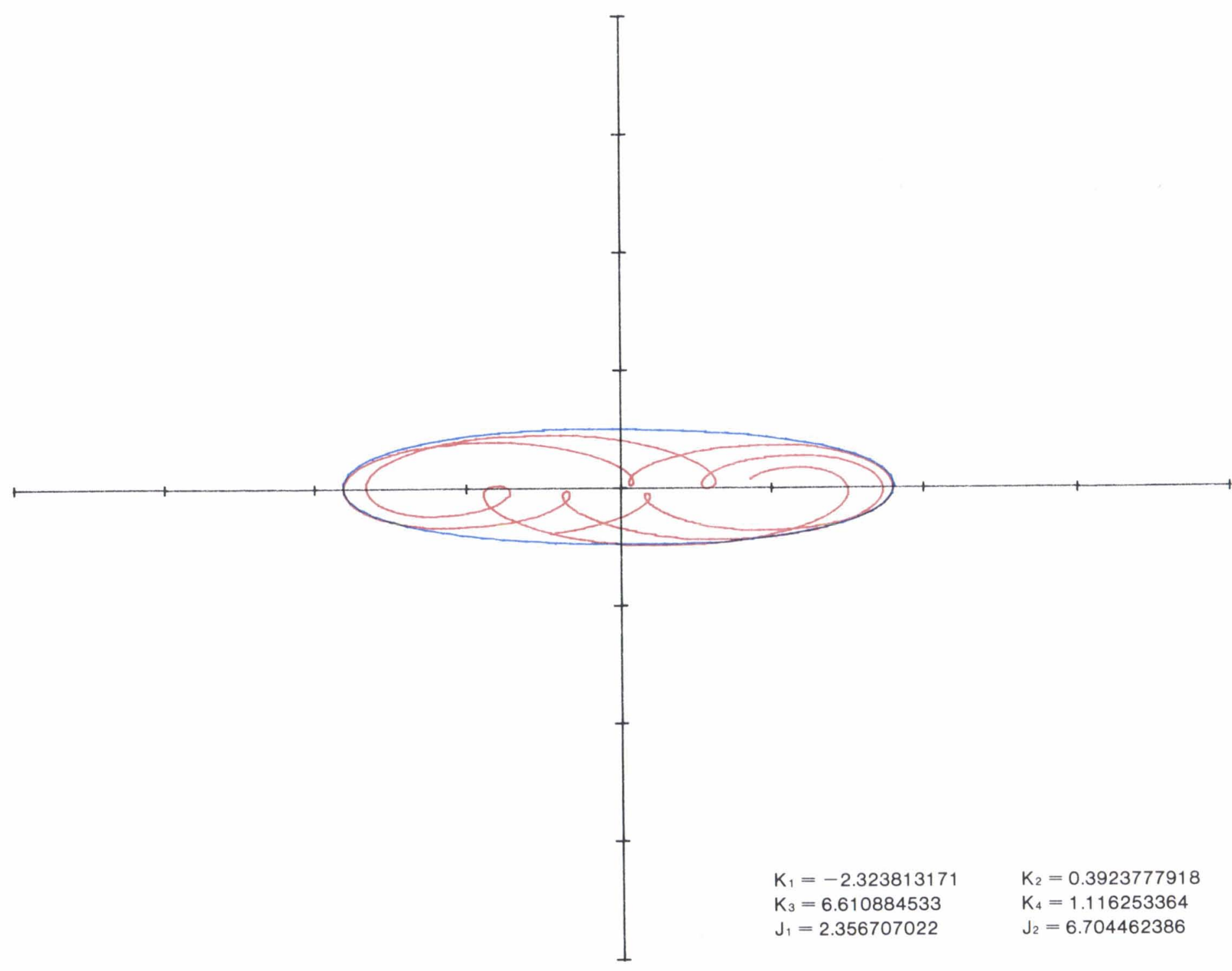

(b2) $\alpha=1, \beta=45^{\circ}, \gamma=45^{\circ}$.

FIGURE 3.-Continued. 


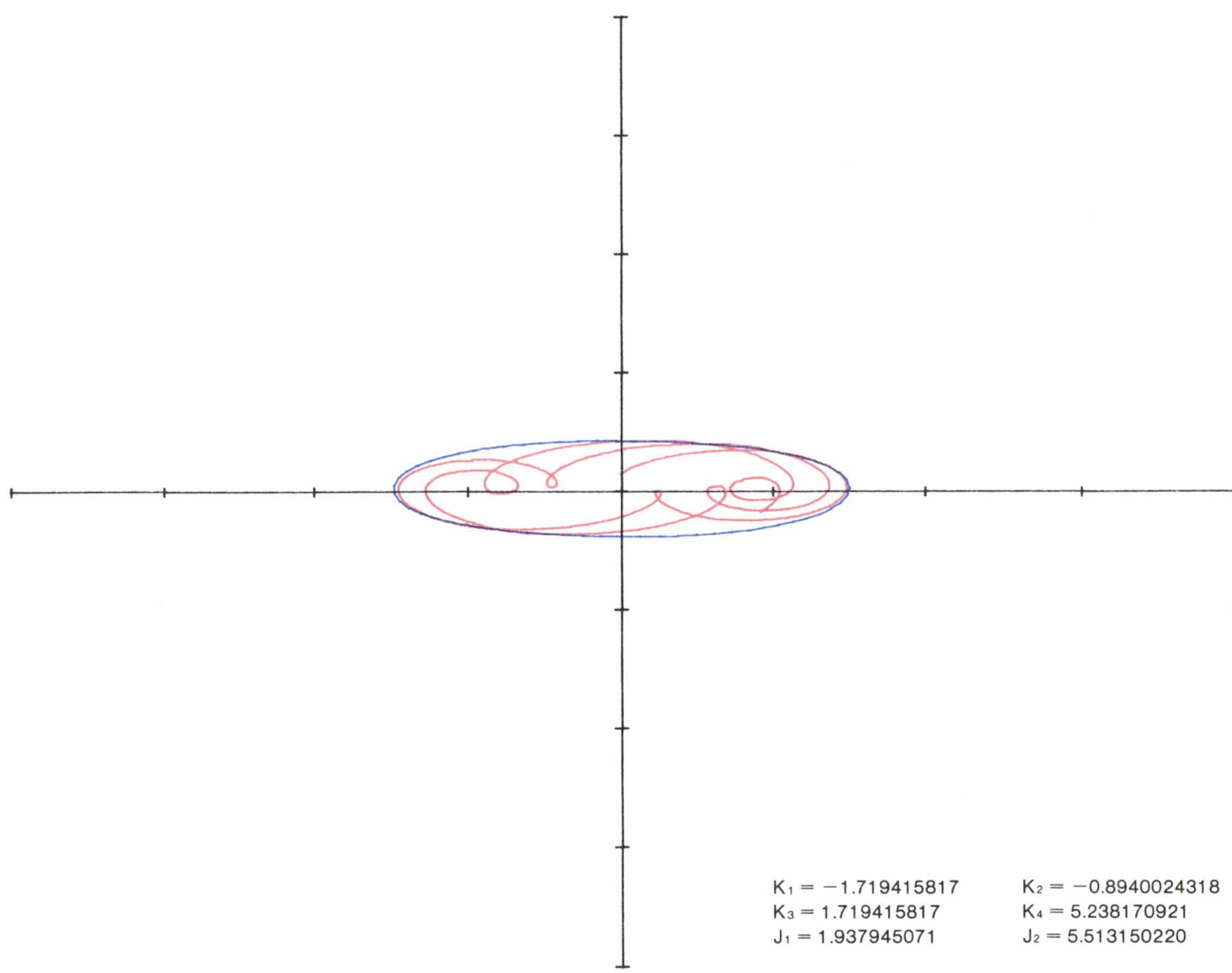

(b3) $\alpha=1, \beta=45^{\circ}, \gamma=90^{\circ}$.

FIGURE 3.-Continued. 


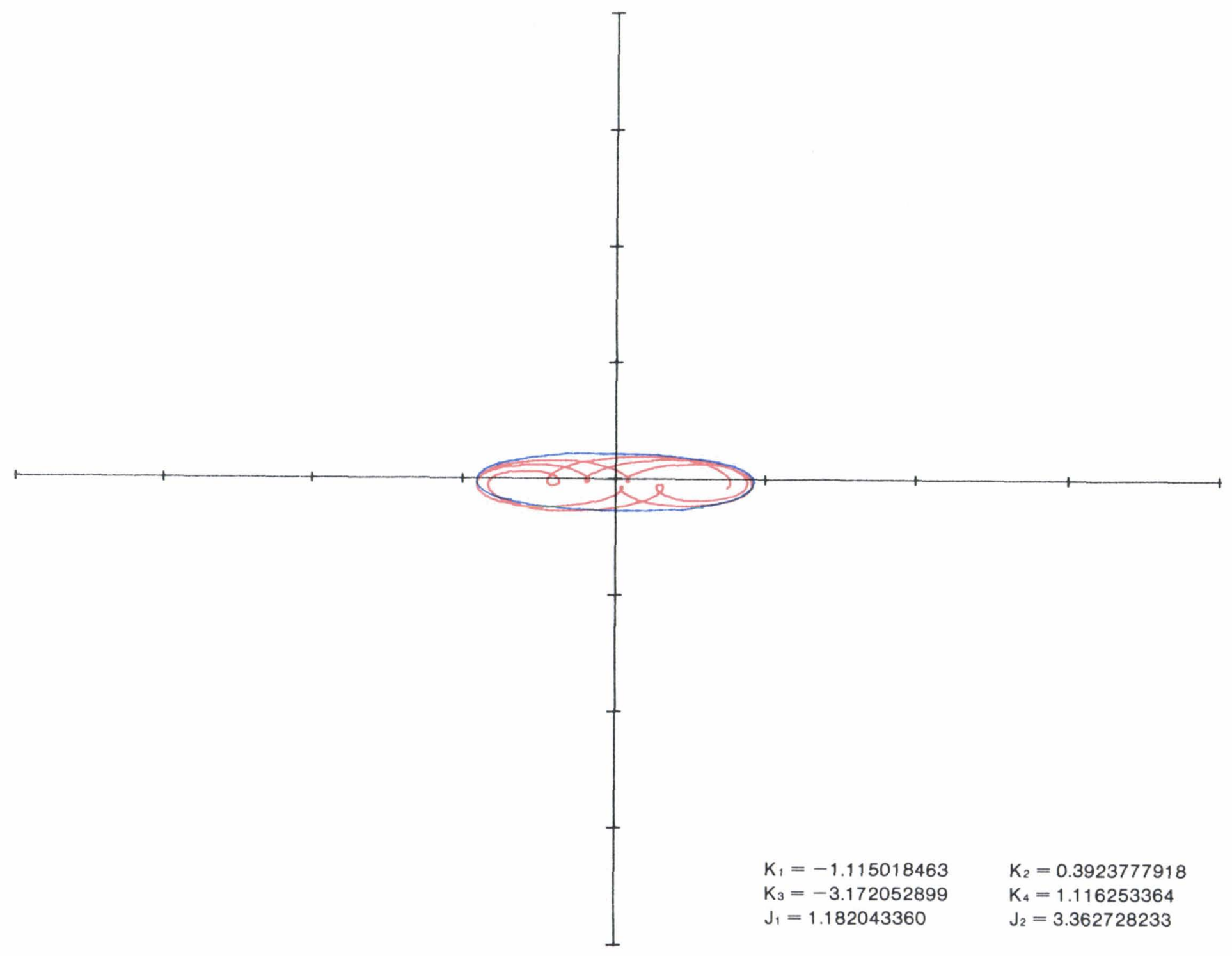

(b4) $\alpha=1, \beta=45^{\circ}, \gamma=135^{\circ}$. 


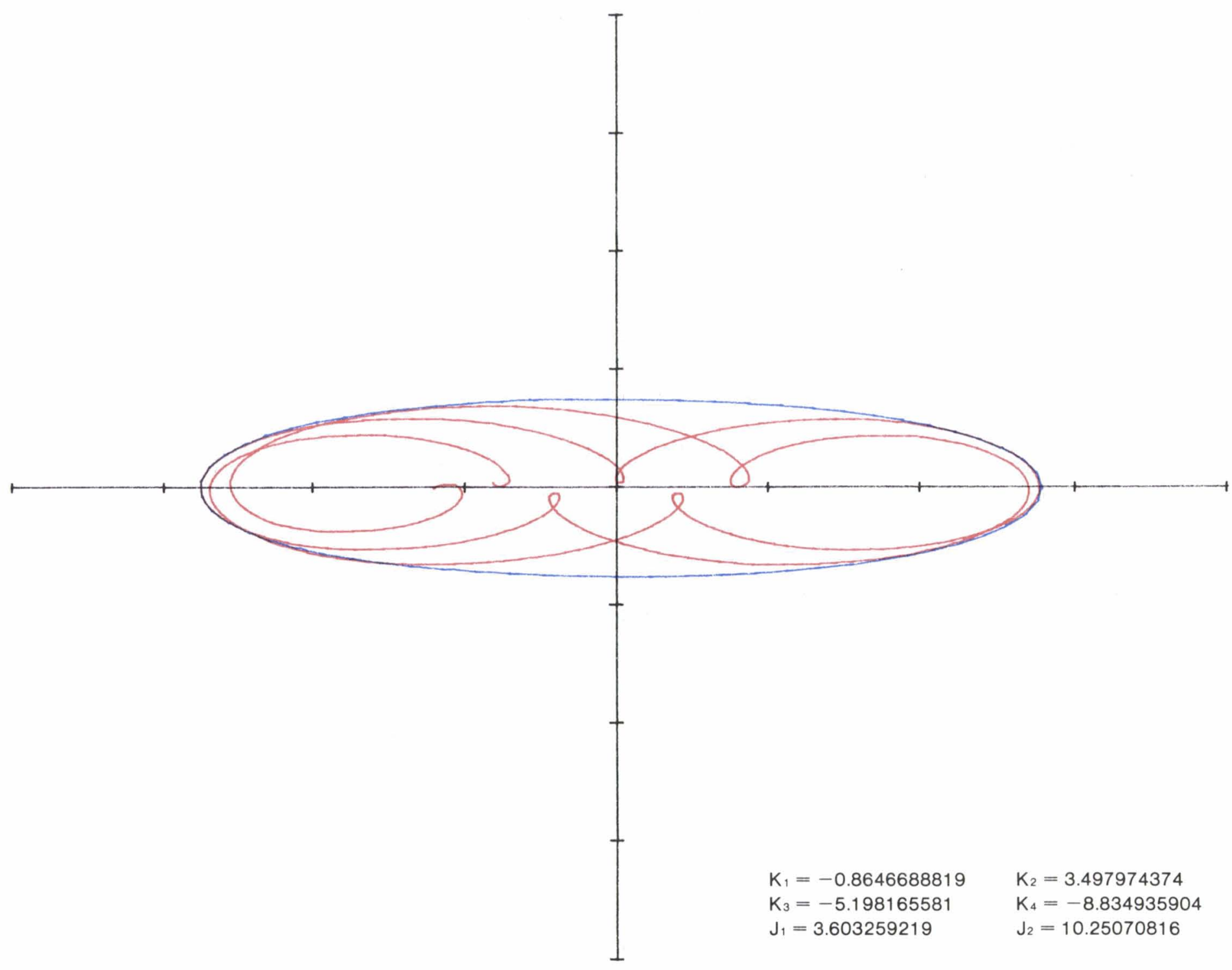

(b5) $\alpha=1, \beta=45^{\circ}, \gamma=180^{\circ}$.

FIGURE 3.-Continued. 


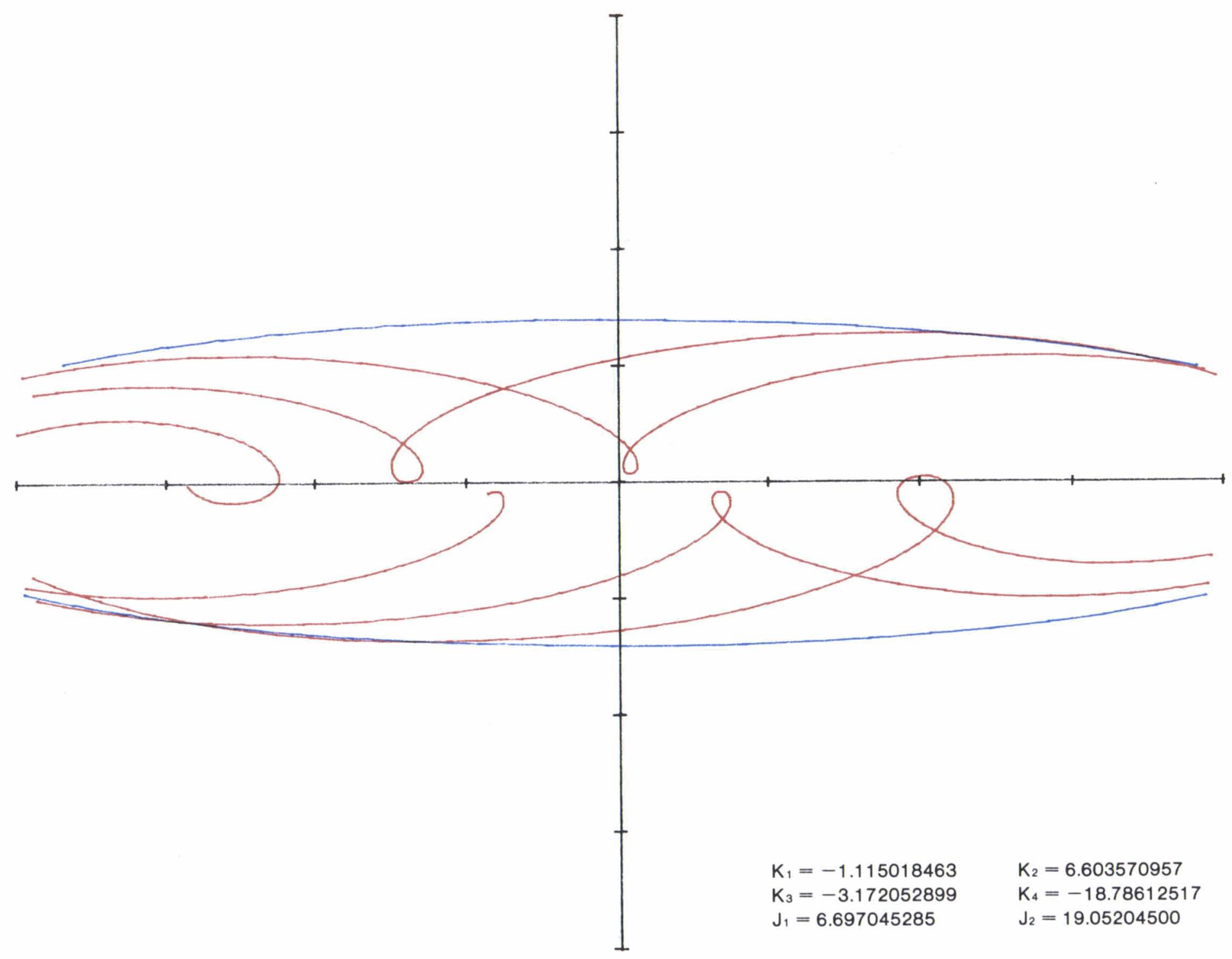

(b6) $\alpha=1, \beta=45^{\circ}, \gamma=225^{\circ}$. 


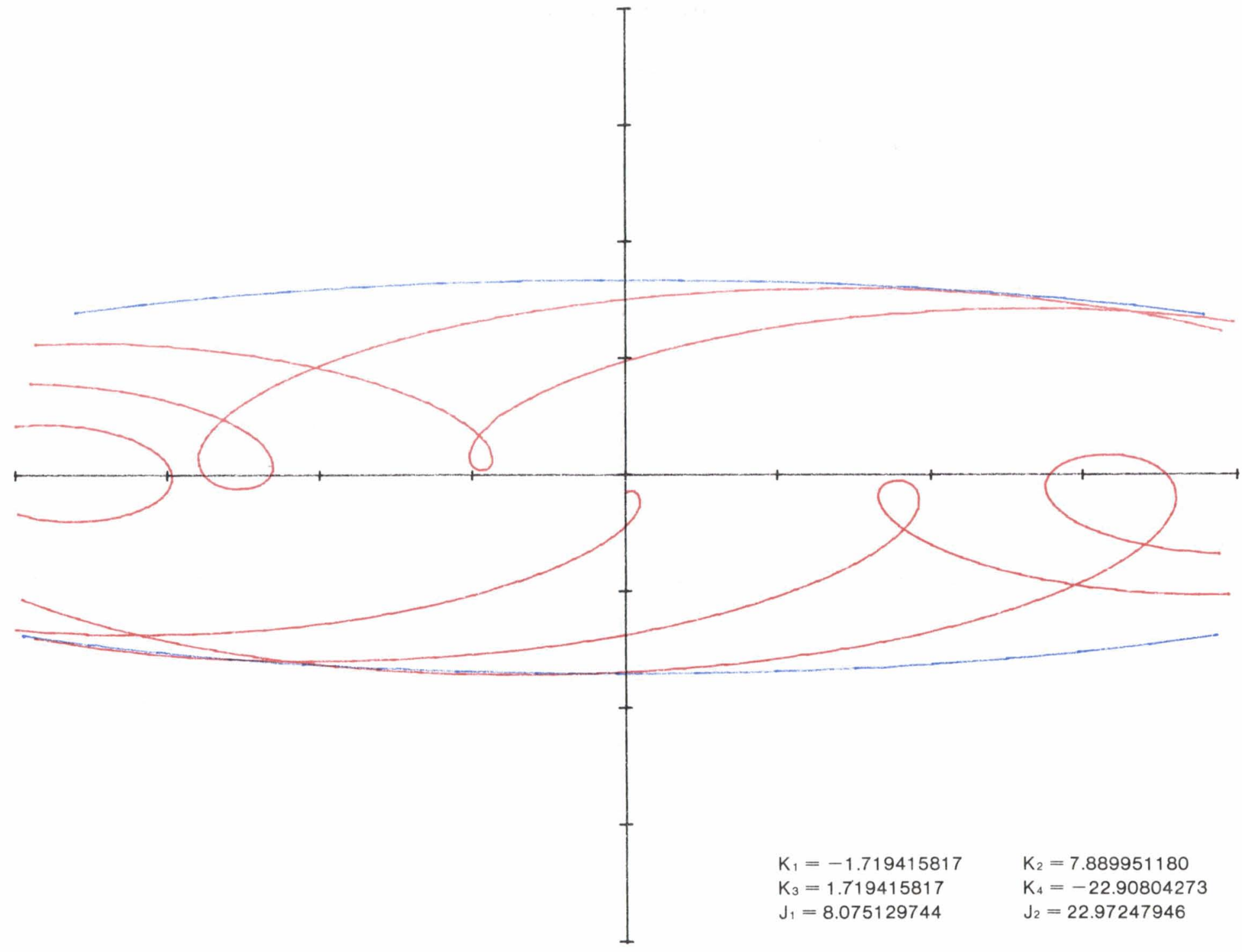

(b7) $\alpha=1, \beta=45^{\circ}, \gamma=270^{\circ}$.

FIGURE 3.-Continued. 


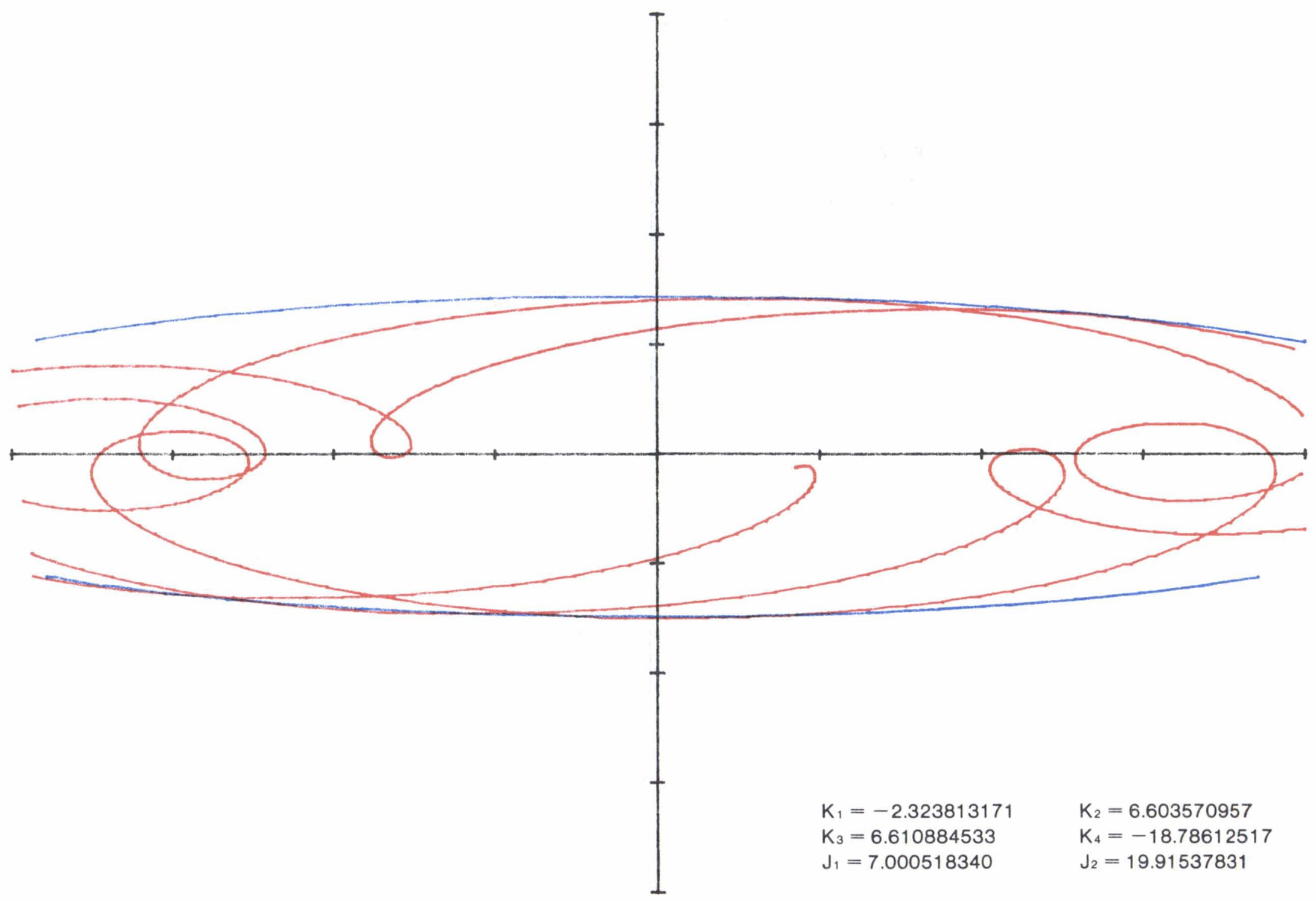

(b8) $\alpha=1, \beta=45^{\circ}, \gamma=315^{\circ}$. 


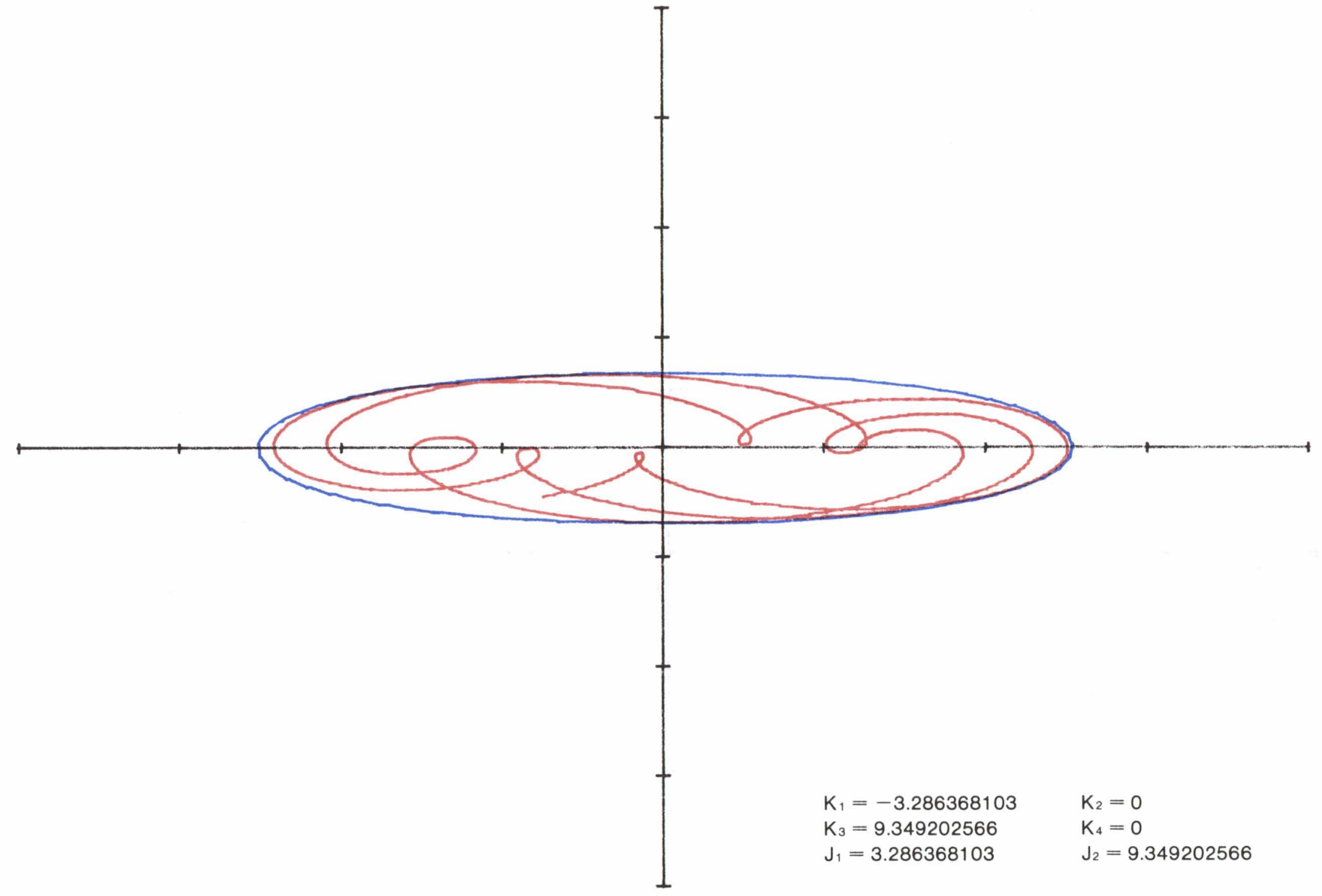

(c1) $\alpha=1, \beta=90^{\circ}, \gamma=0^{\circ}$.

FIGURE 3.-Continued. 


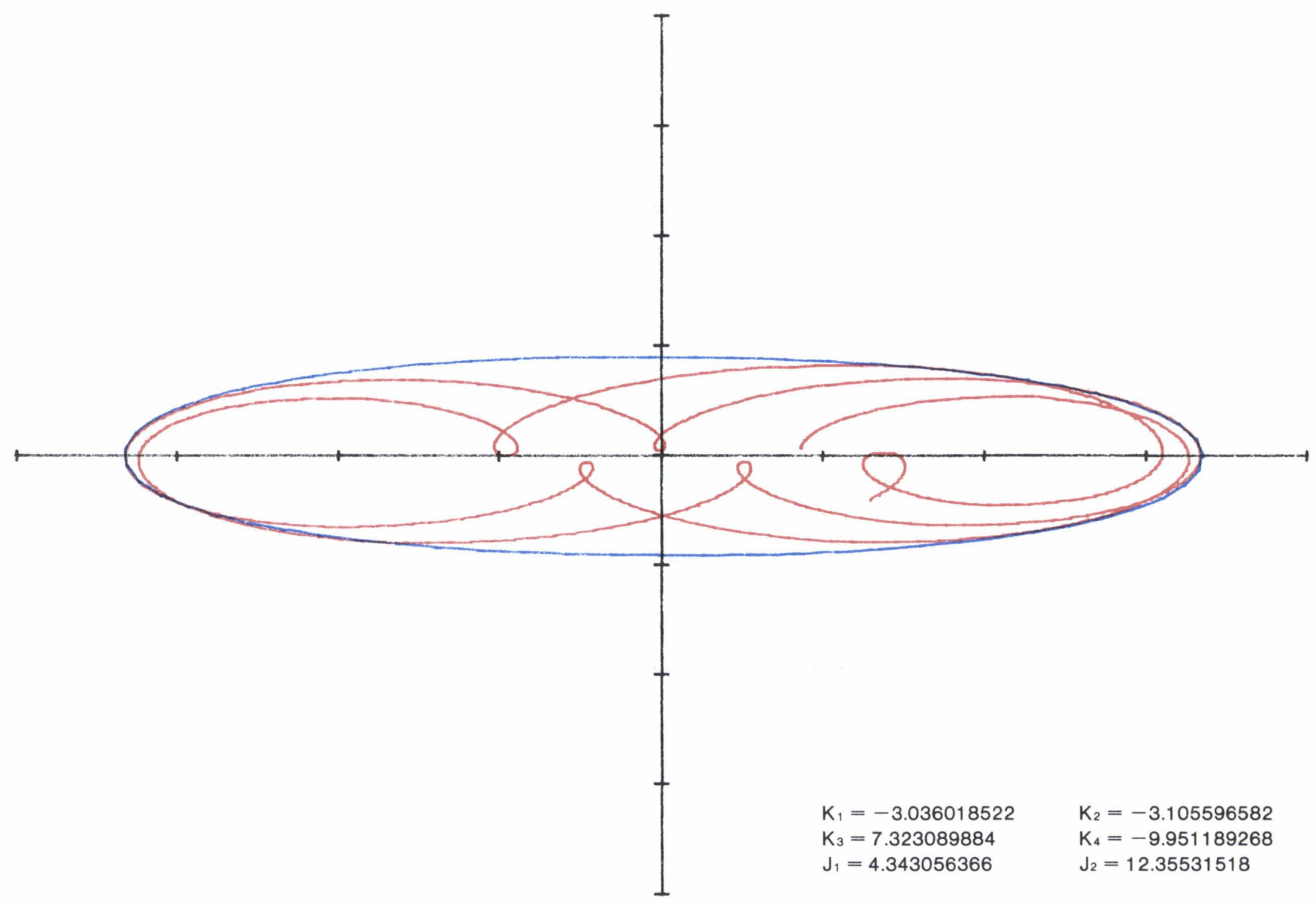

(c2) $\alpha=1, \beta=90^{\circ}, \gamma=45^{\circ}$. 


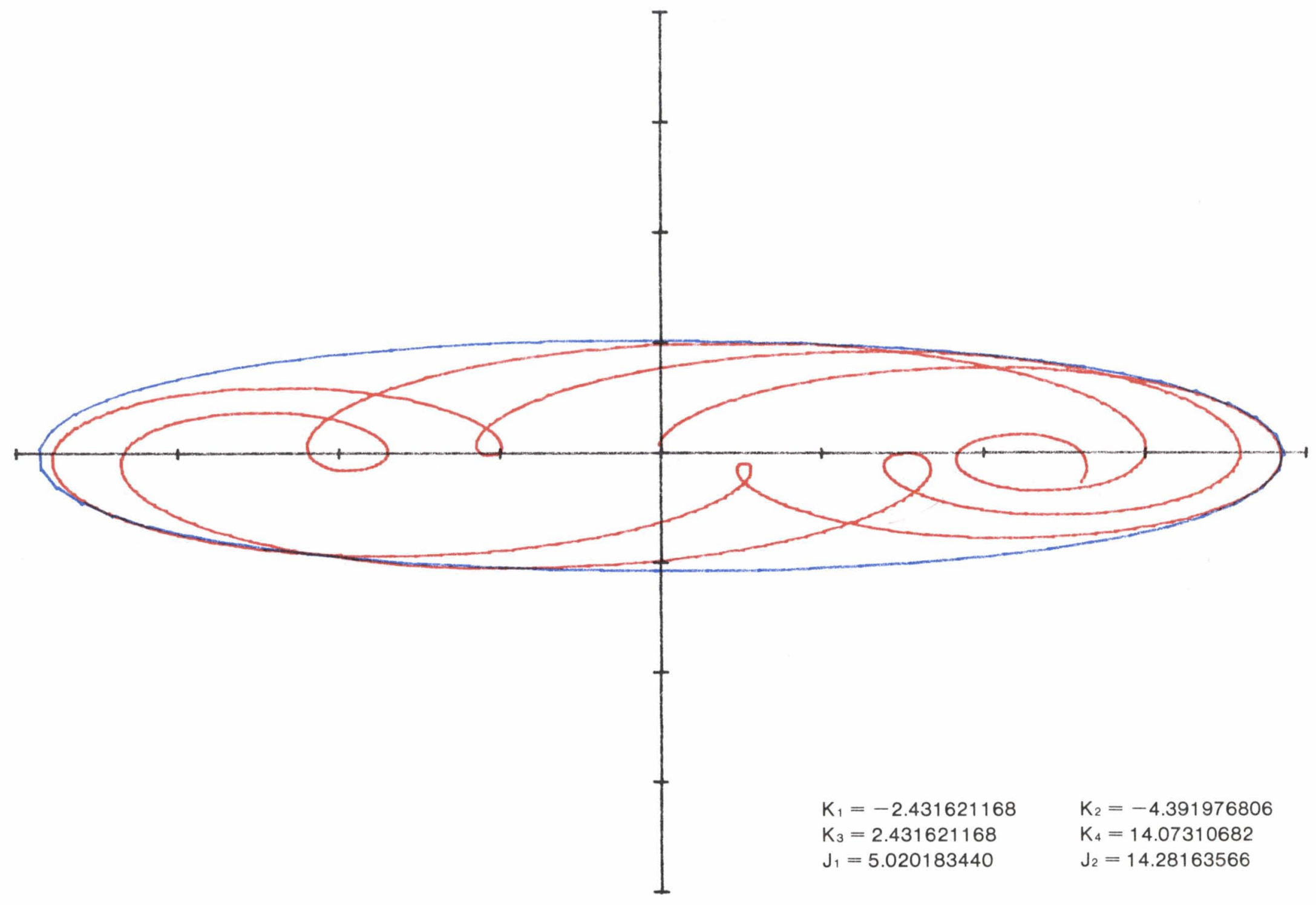

(c3) $\alpha=1, \beta=90^{\circ}, \gamma=90^{\circ}$.

FIGURE 3.-Continued. 


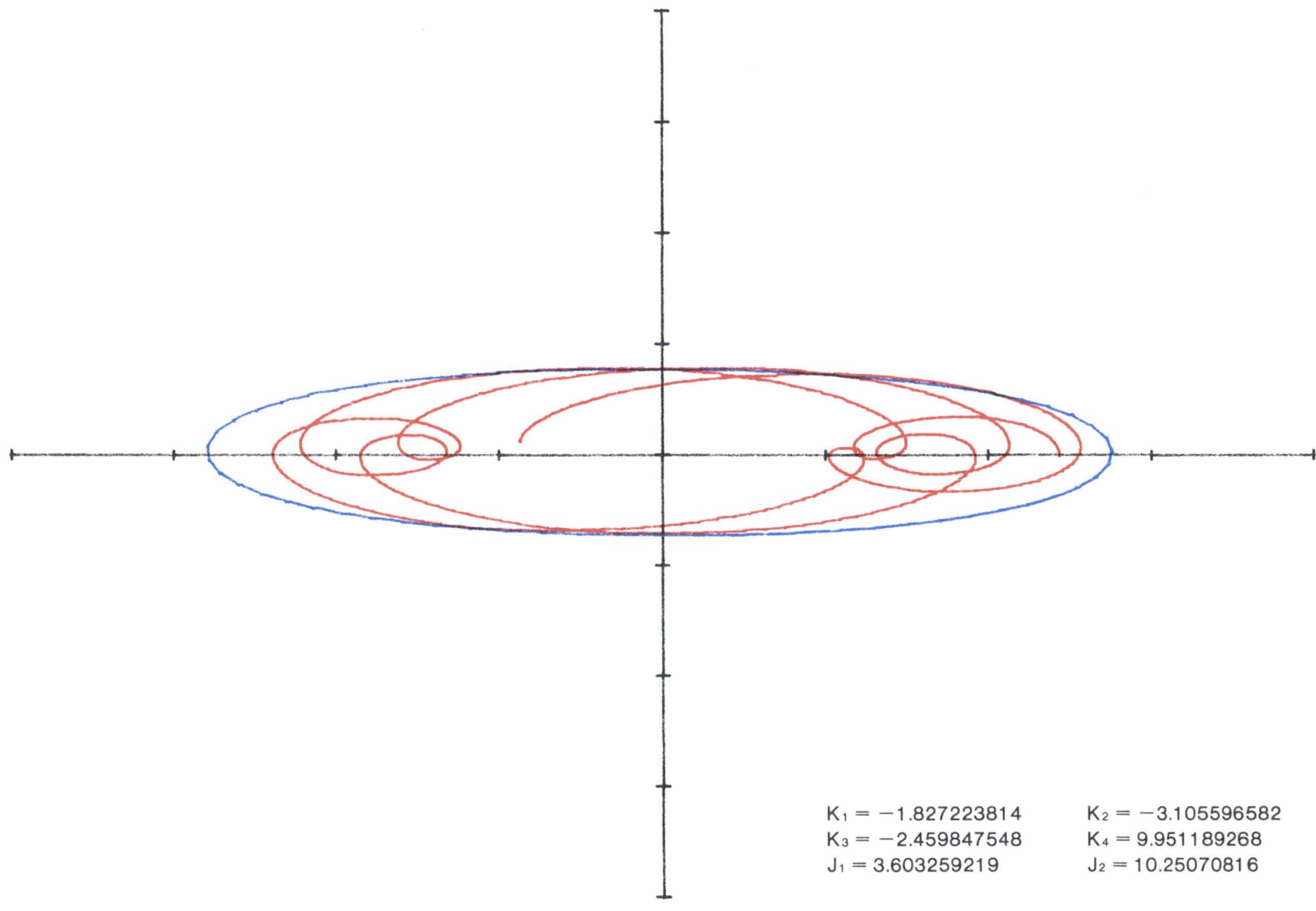

(c4) $\alpha=1, \beta=90^{\circ}, \gamma=135^{\circ}$. 


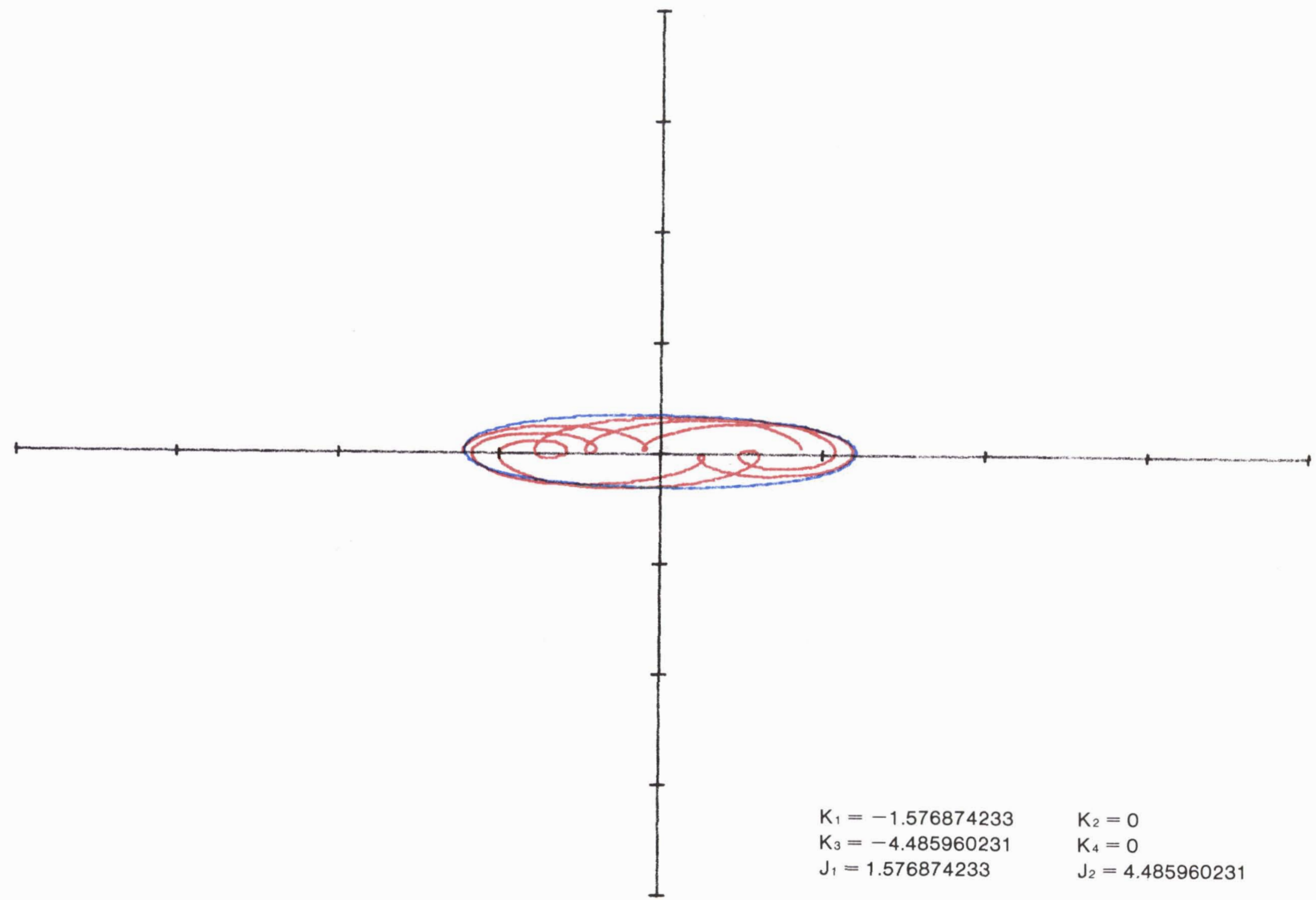

(c5) $\alpha=1, \beta=90^{\circ}, \gamma=180^{\circ}$.

FIGURE 3.-Continued. 


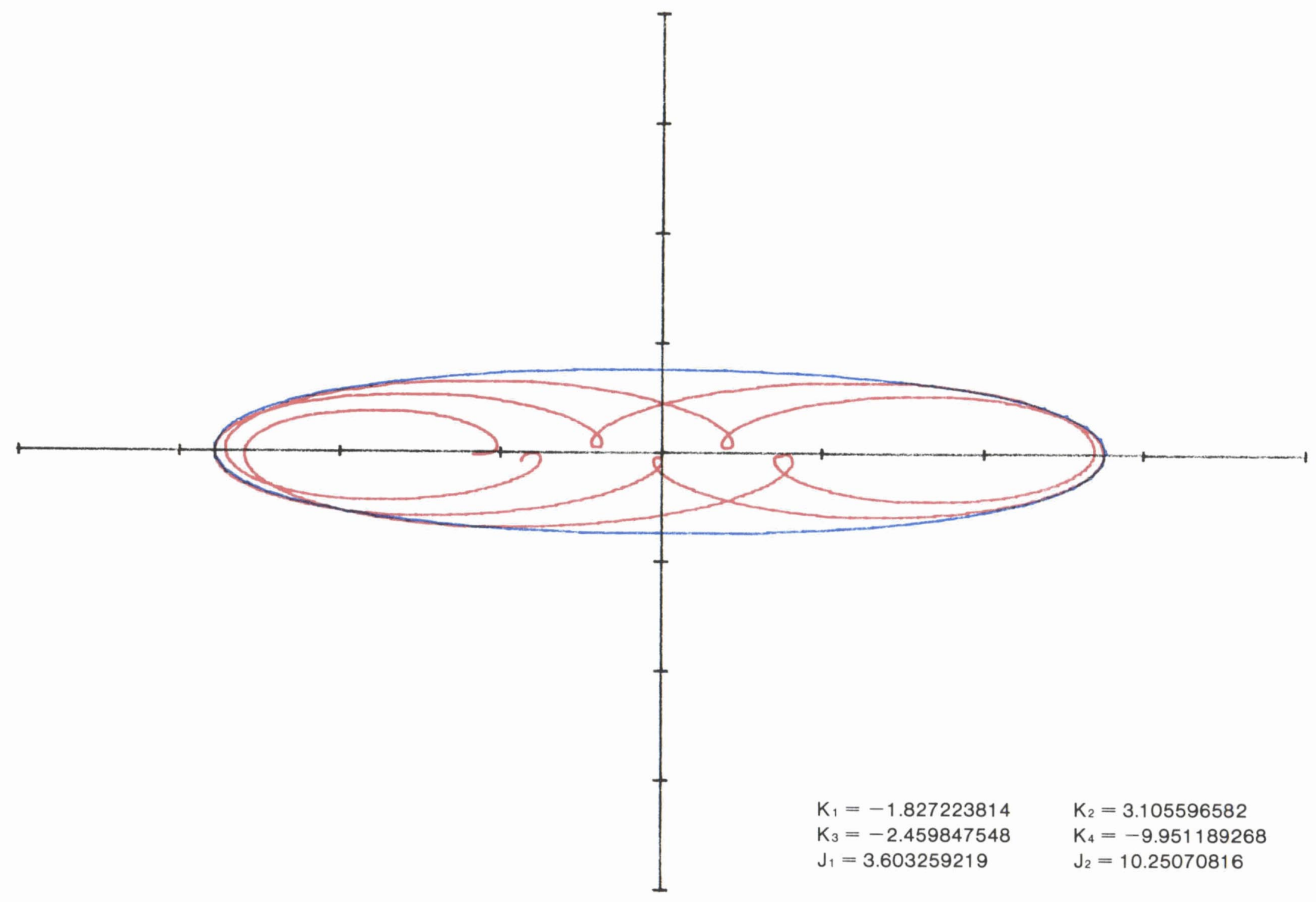

(c6) $\alpha=1, \beta=90^{\circ}, \gamma=225^{\circ}$. 


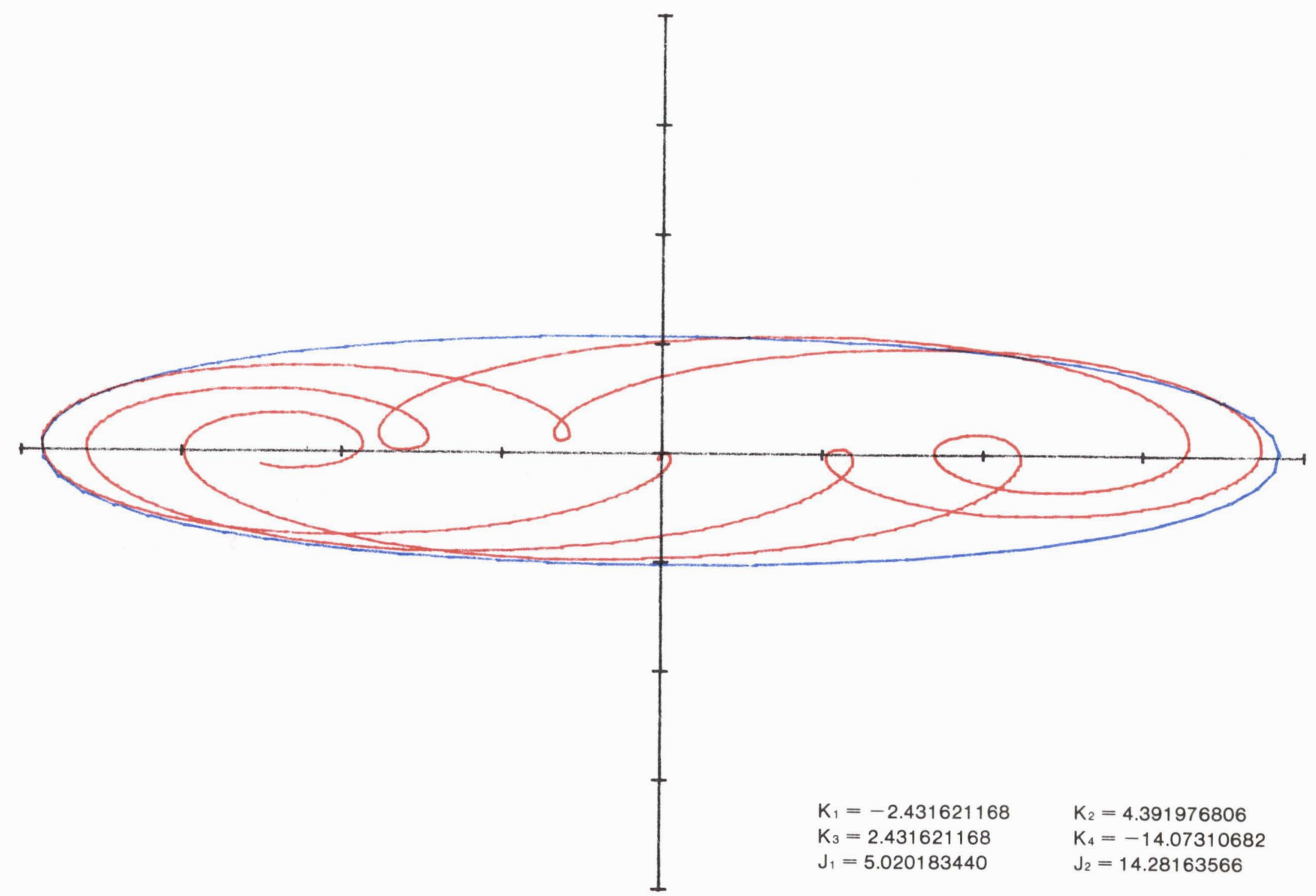

(c7) $\alpha=1, \beta=90^{\circ}, \gamma=270^{\circ}$.

FIGURE 3.-Continued. 


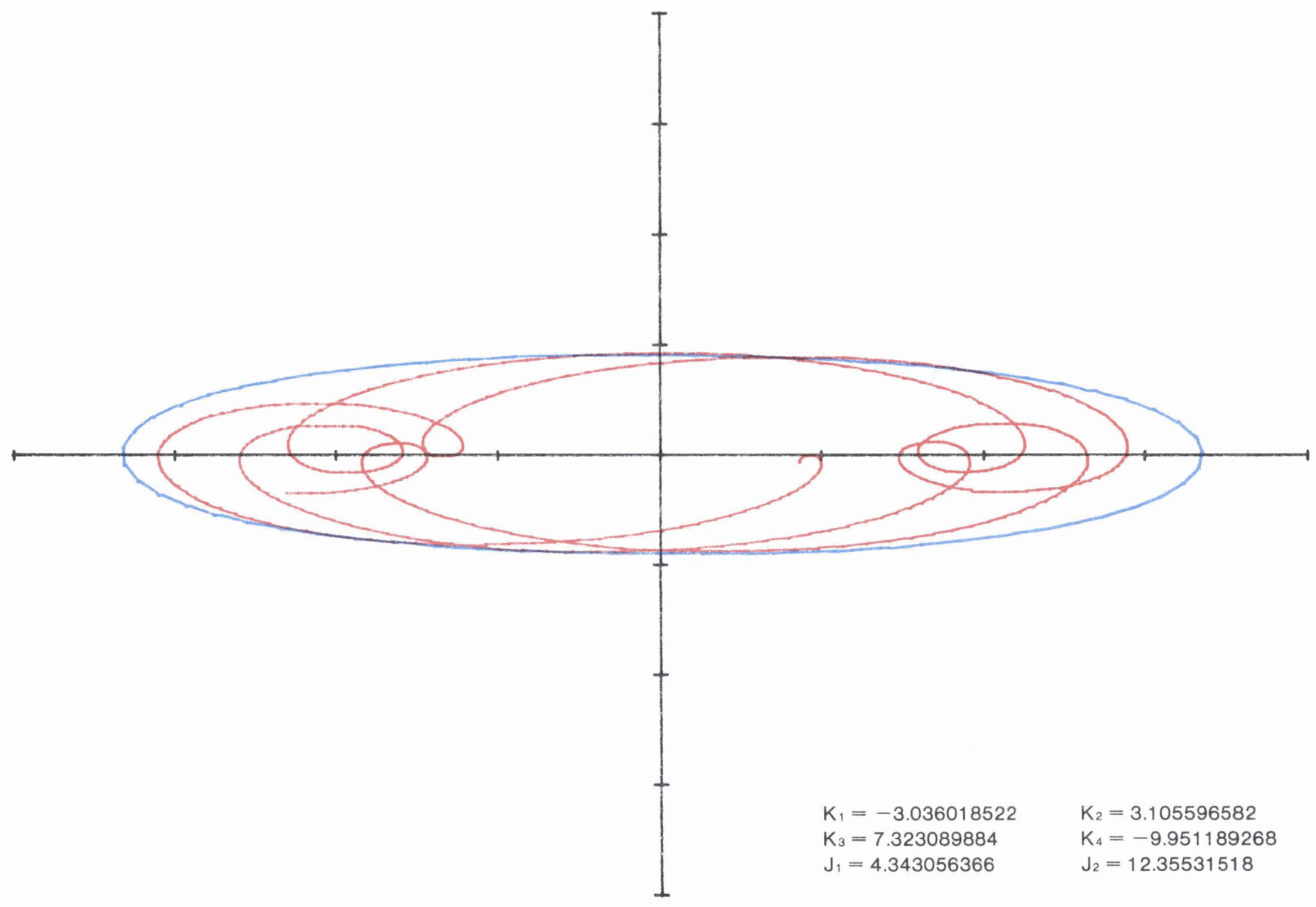

(c8) $\alpha=1, \beta=90^{\circ}, \gamma=315^{\circ}$. 


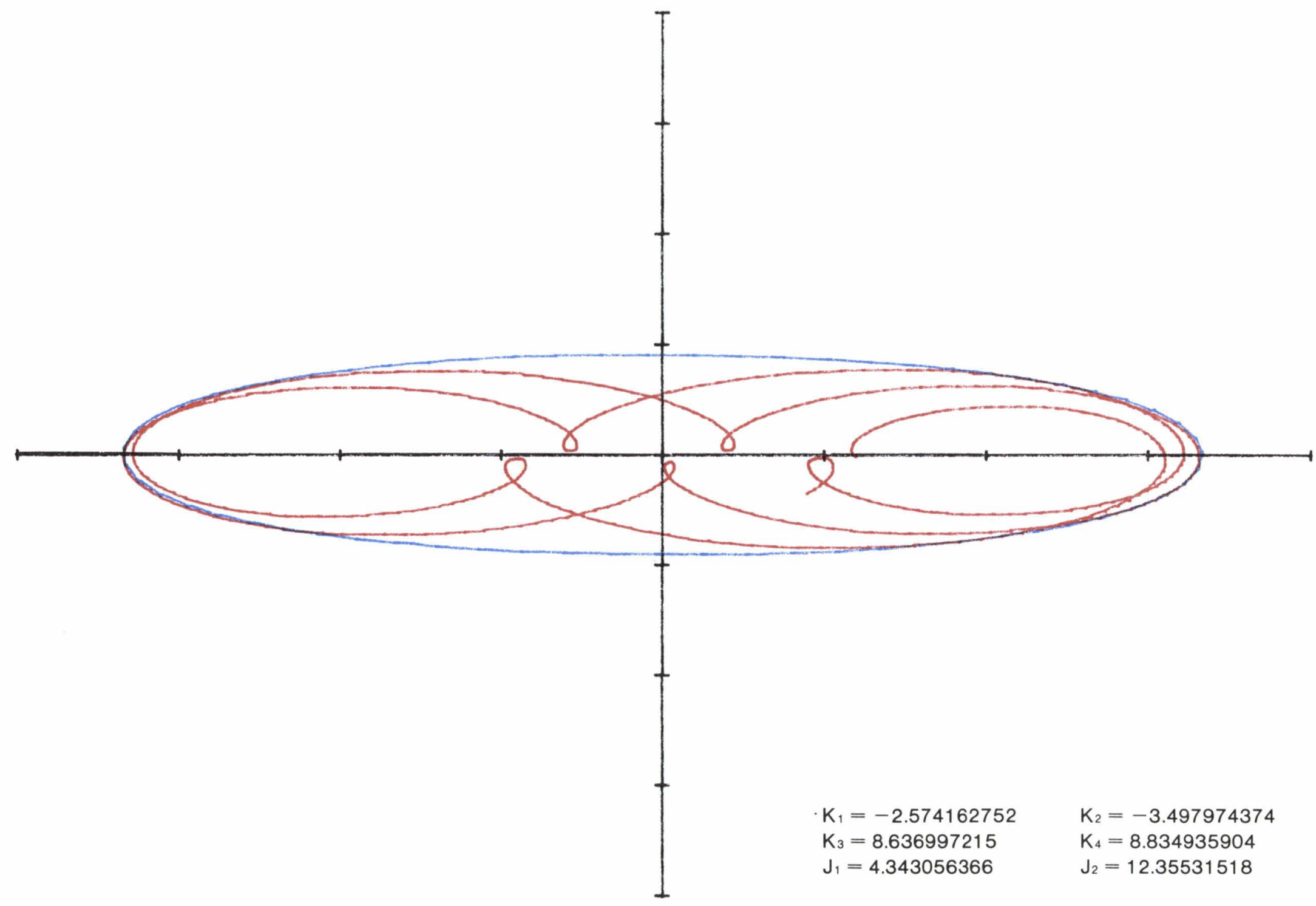

(d1) $\alpha=1, \beta=135^{\circ}, \gamma=0^{\circ}$.

FIGURE 3.-Continued. 


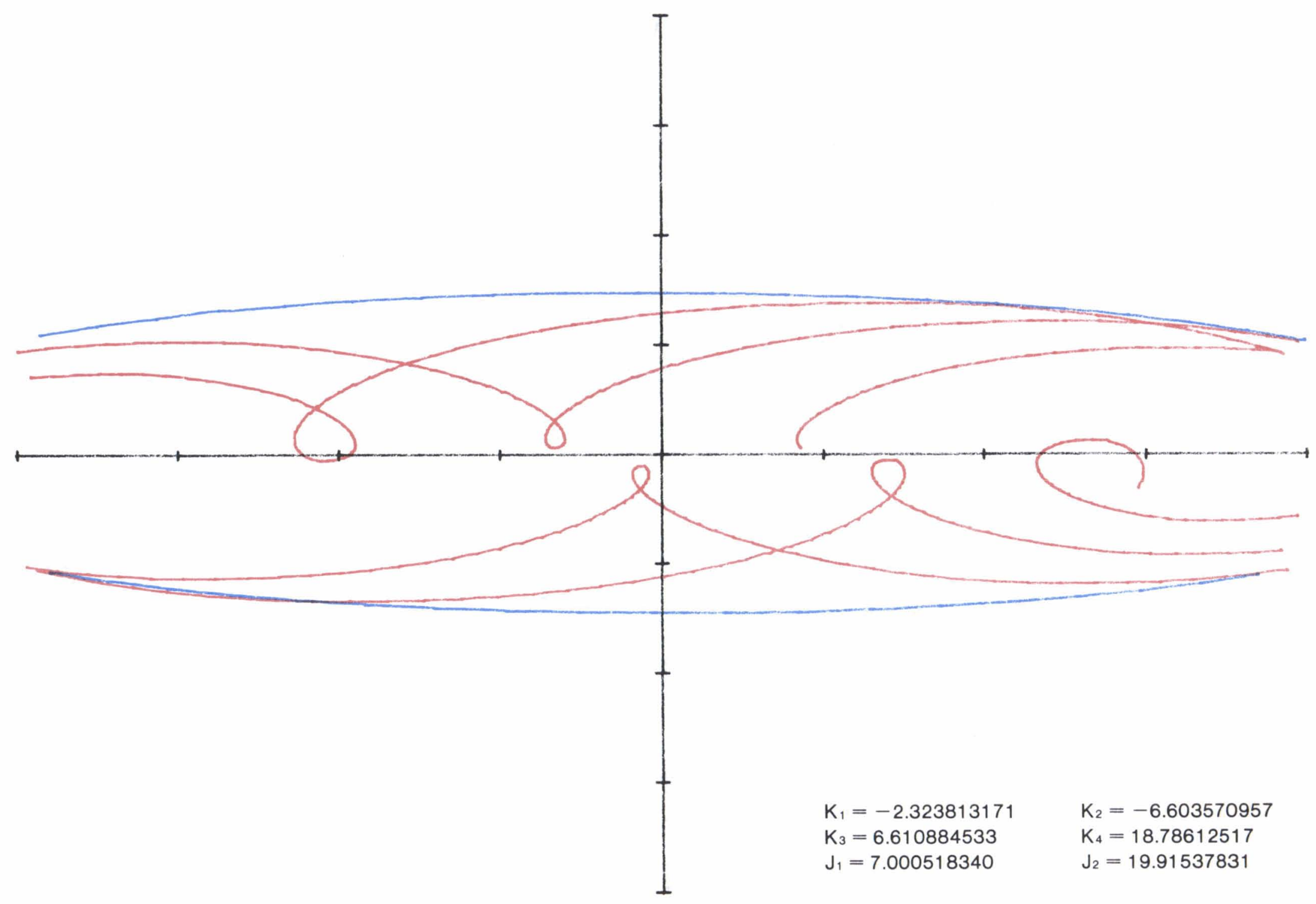

(d2) $\alpha=1, \beta=135^{\circ}, \gamma=45^{\circ}$. 


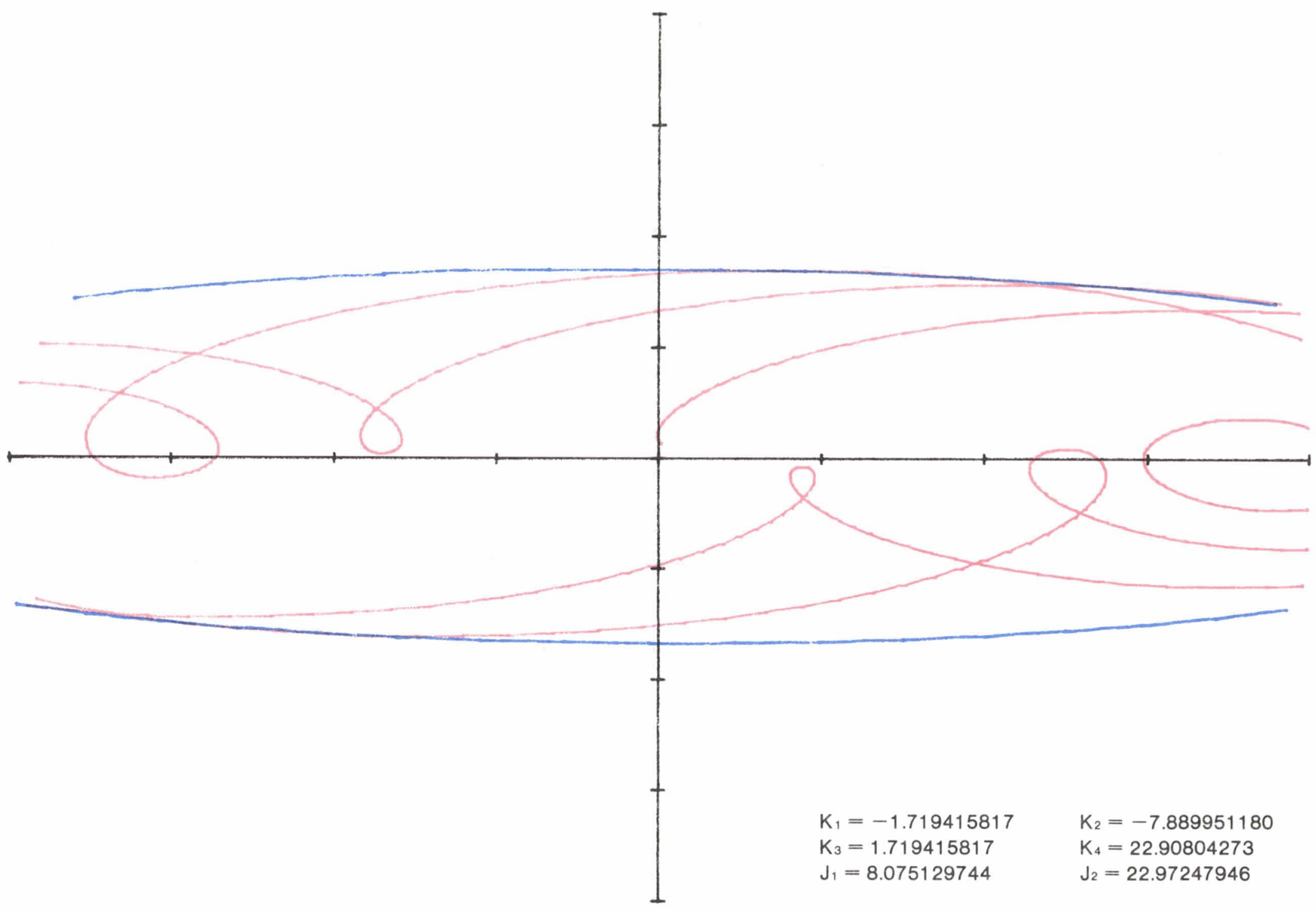

(d3) $\alpha=1, \beta=135^{\circ}, \gamma=90^{\circ}$.

FIGURE 3.-Continued. 


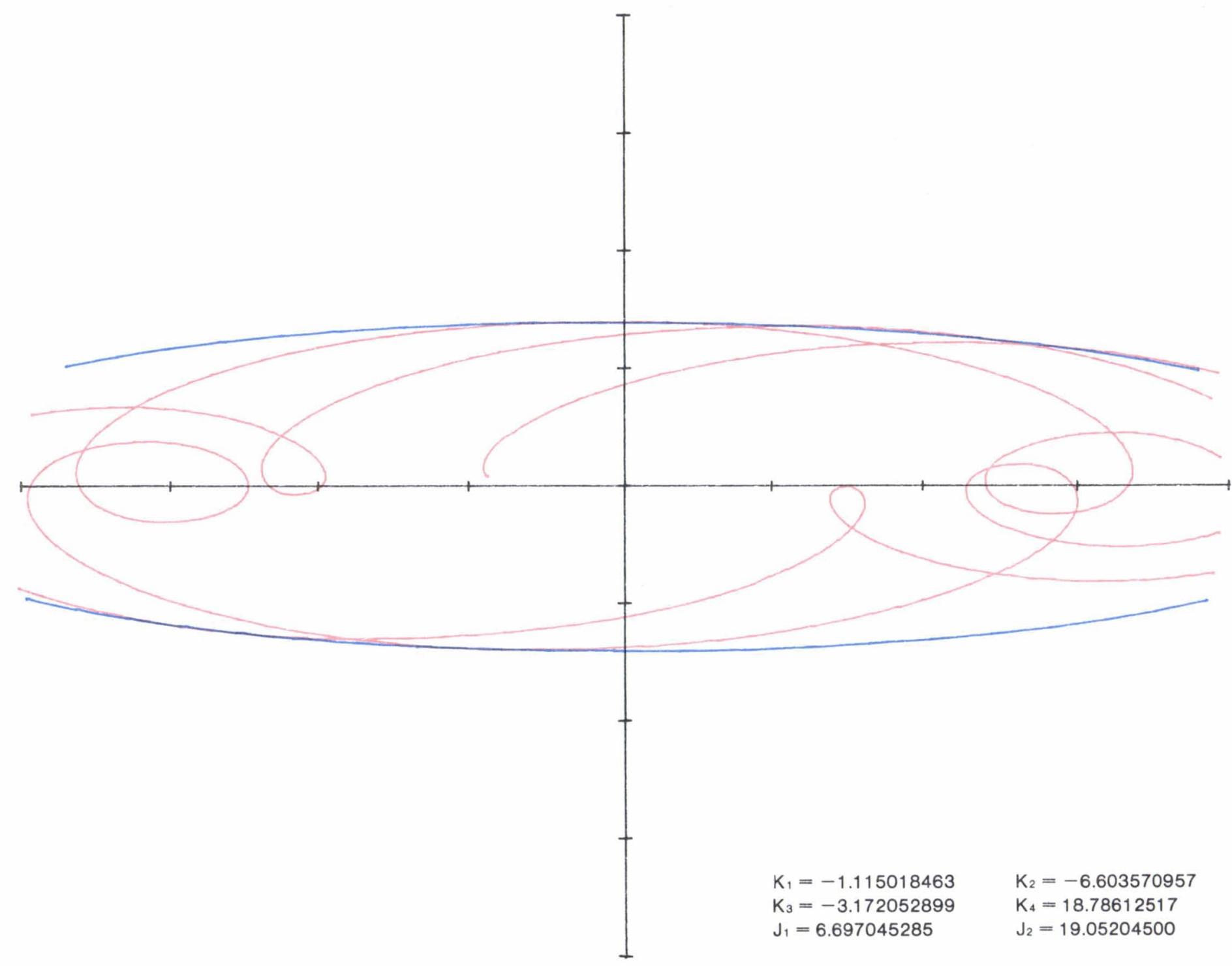

(d4) $\alpha=1, \beta=135^{\circ}, \gamma=135^{\circ}$. 


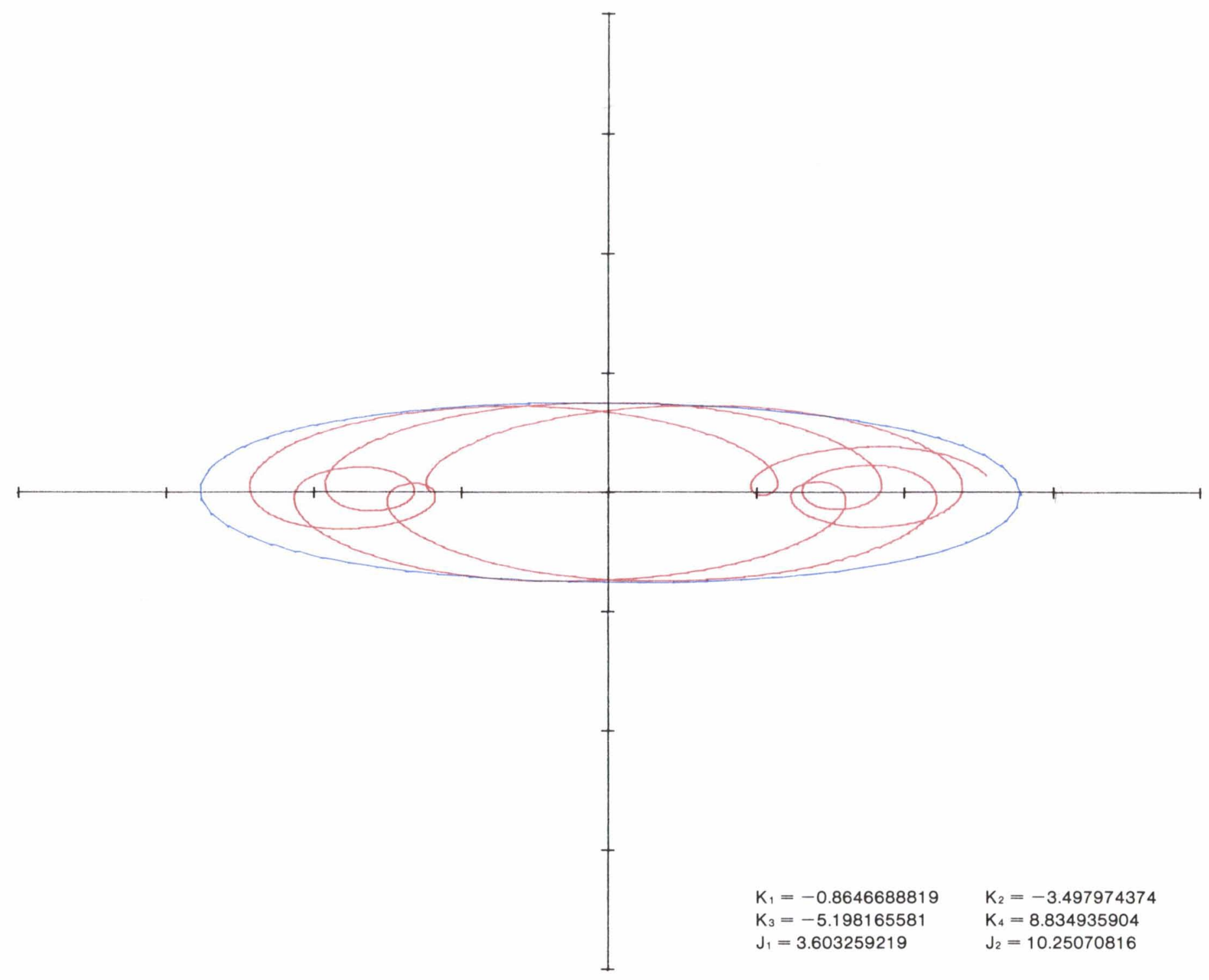

(d5) $\alpha=1, \beta=135^{\circ}, \gamma=180^{\circ}$.

FIGURE 3.-Continued. 


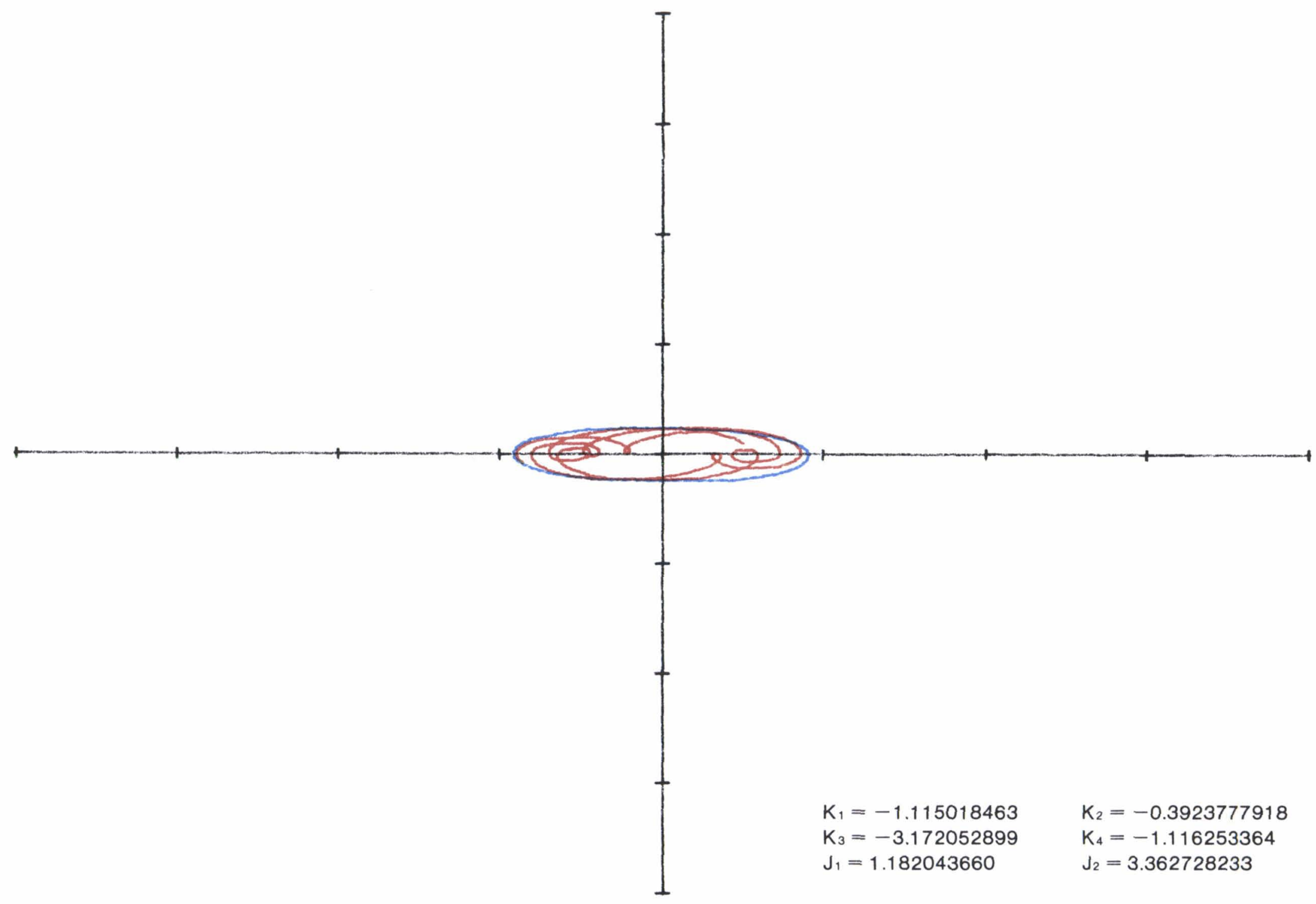

(d6) $\alpha=1, \beta=135^{\circ}, \gamma=225^{\circ}$. 


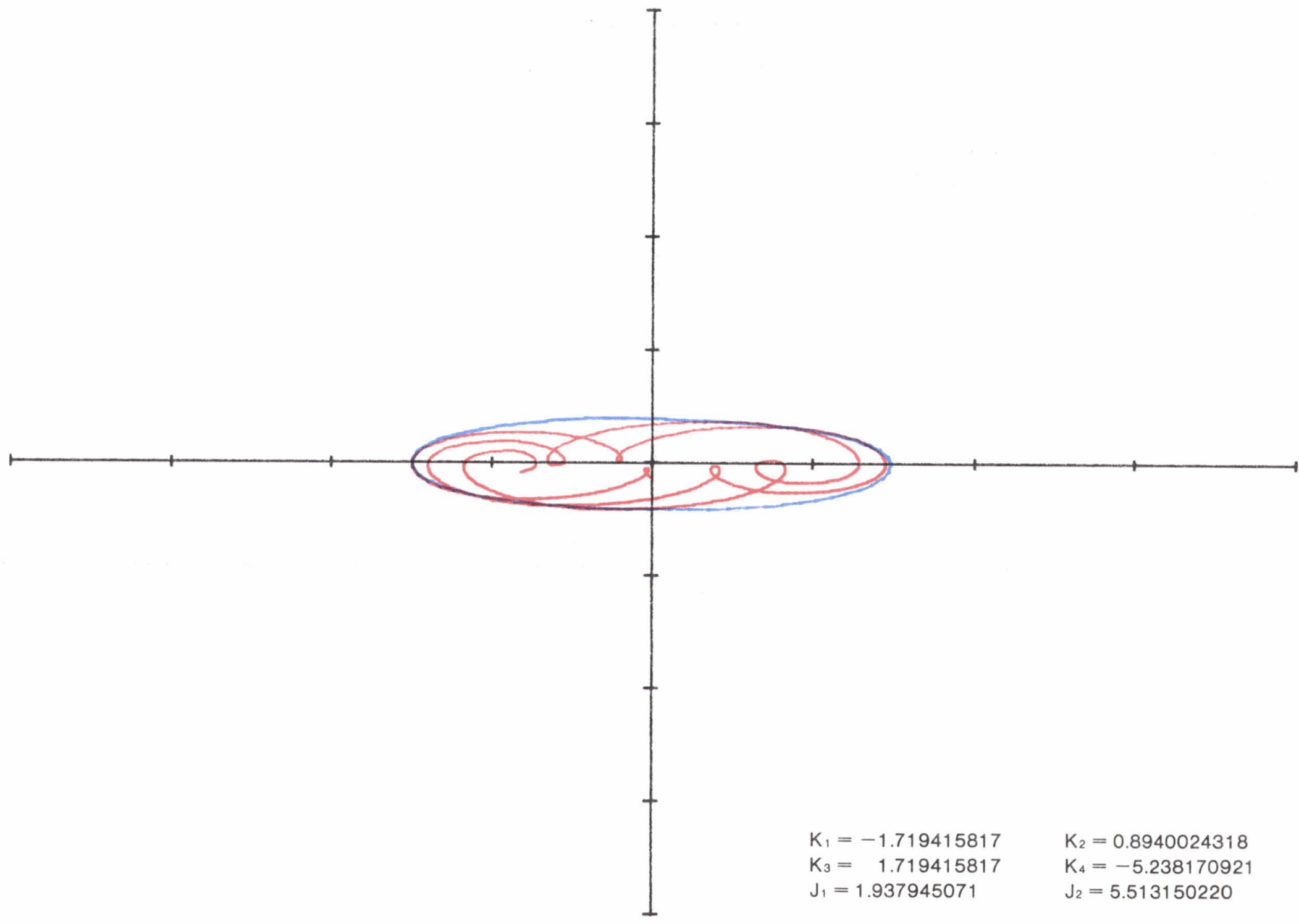

(d7) $\alpha=1, \beta=135^{\circ}, \gamma=270^{\circ}$.

FIGURE 3.-Continued. 


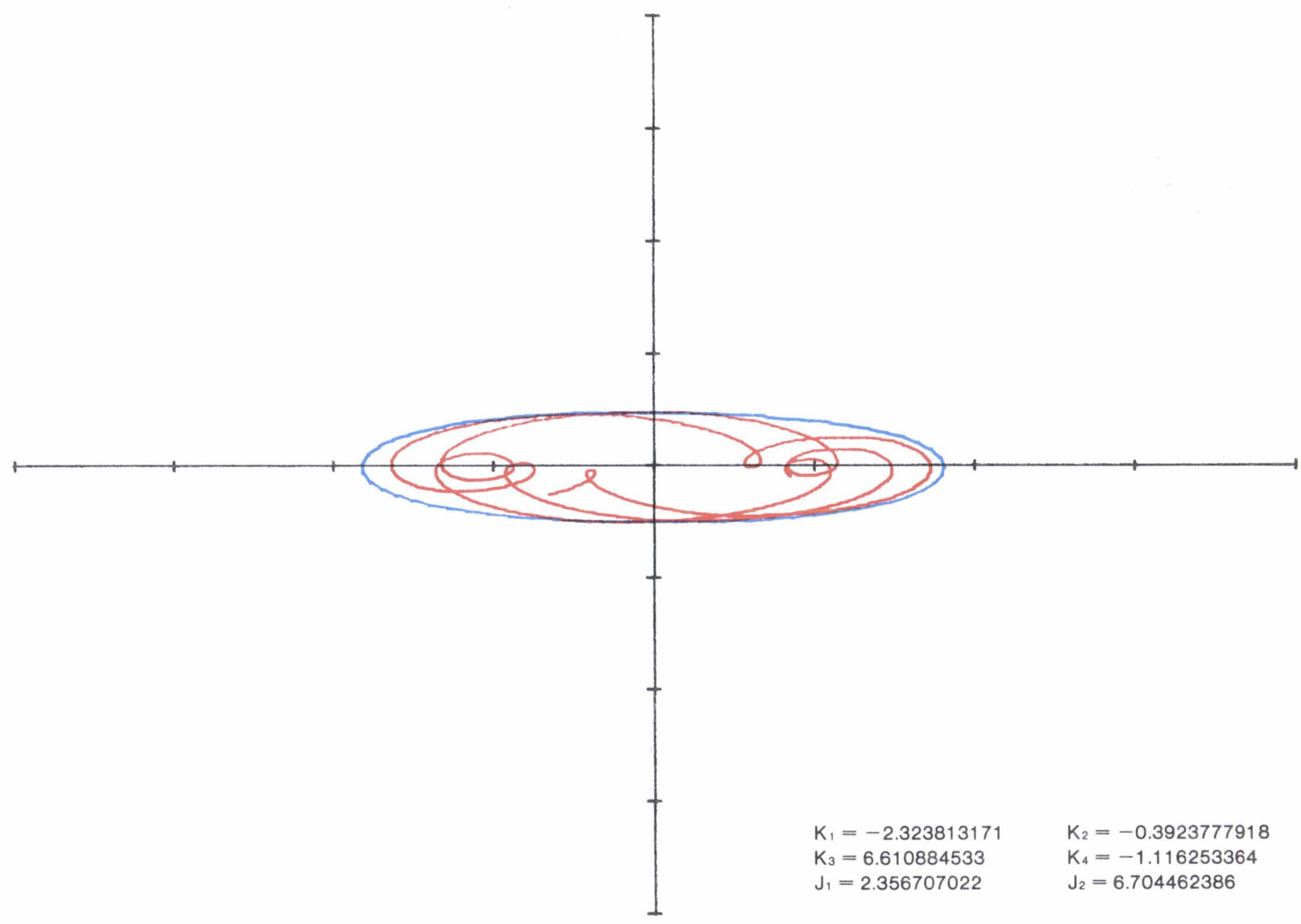

(d8) $\alpha=1, \beta=135^{\circ}, \gamma=315^{\circ}$. 


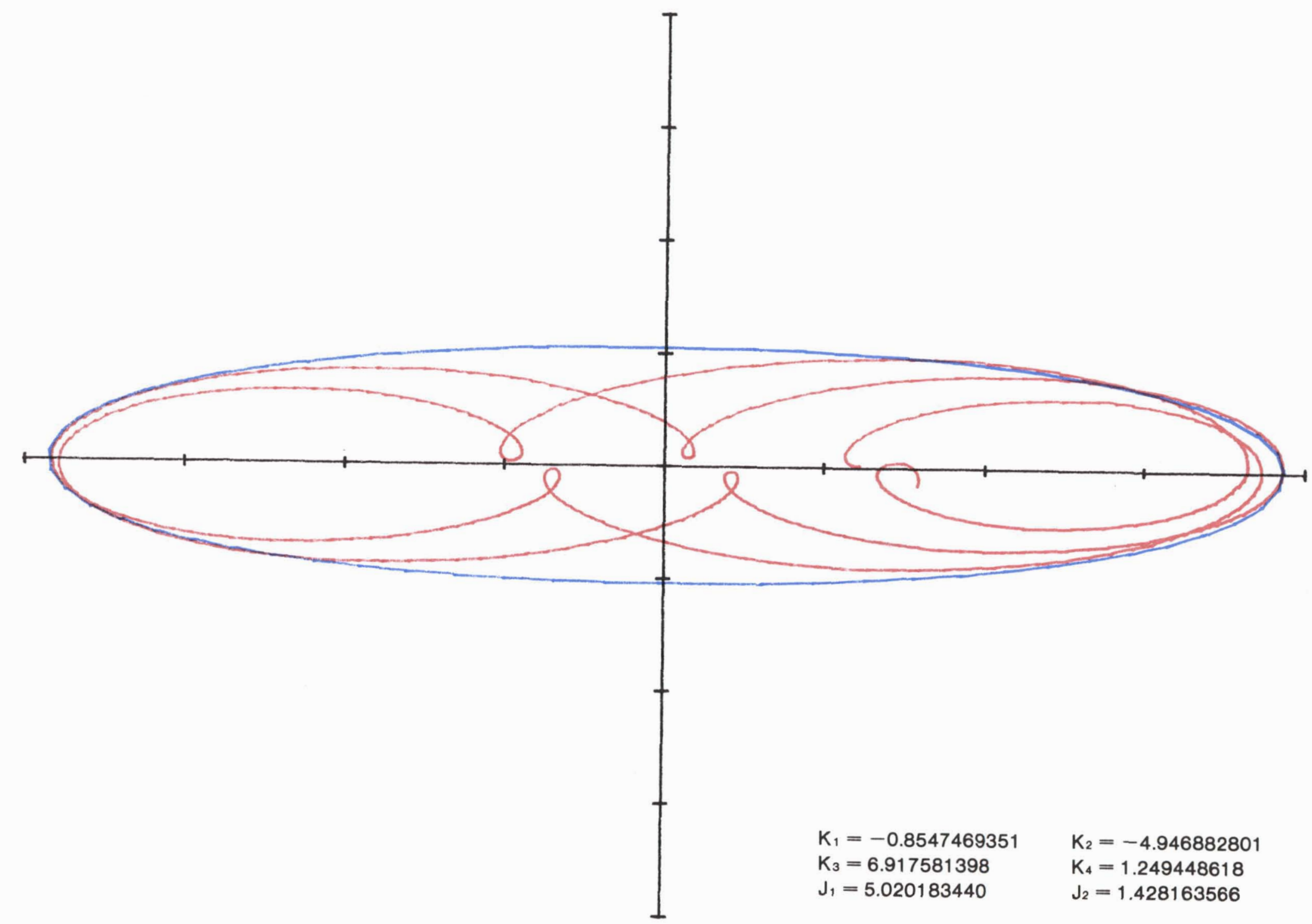

(e1) $\alpha=1, \beta=180^{\circ}, \gamma=0^{\circ}$.

FIGURE 3.-Continued. 


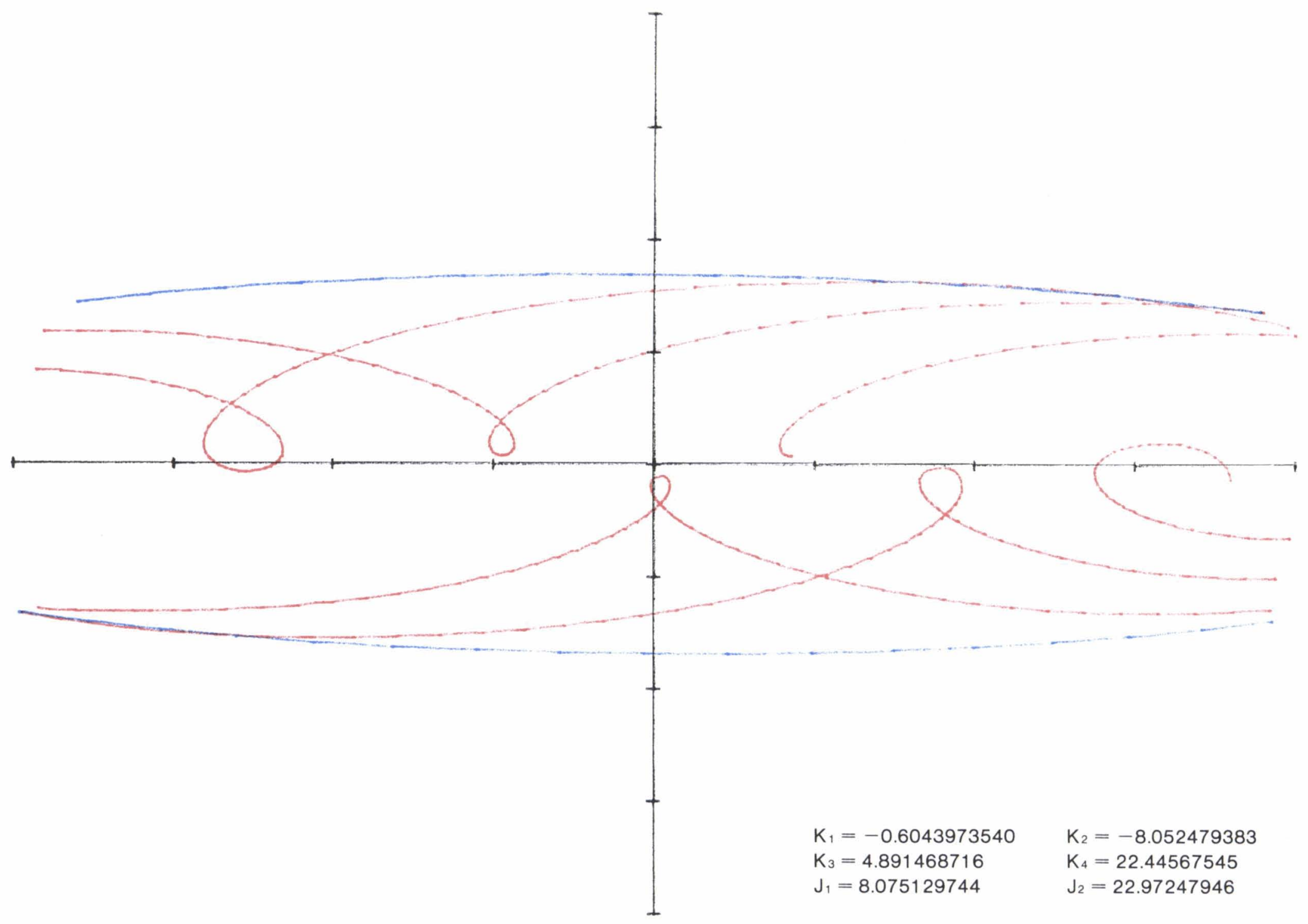

(e2) $\alpha=1, \beta=180^{\circ}, \gamma=45^{\circ}$. 


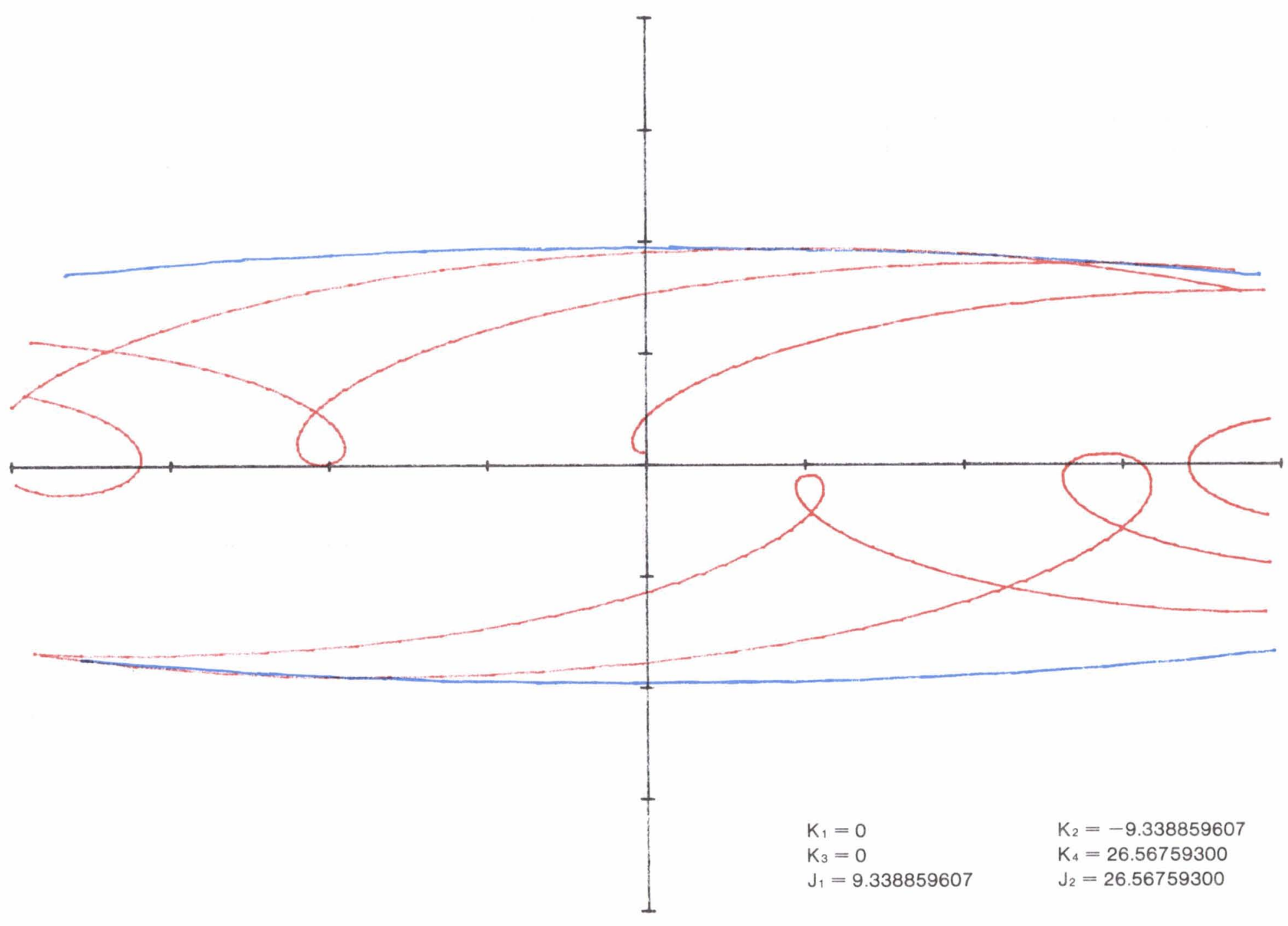

(e3) $\alpha=1, \beta=180^{\circ}, \gamma=90^{\circ}$.

FIGURE 3.-Continued. 


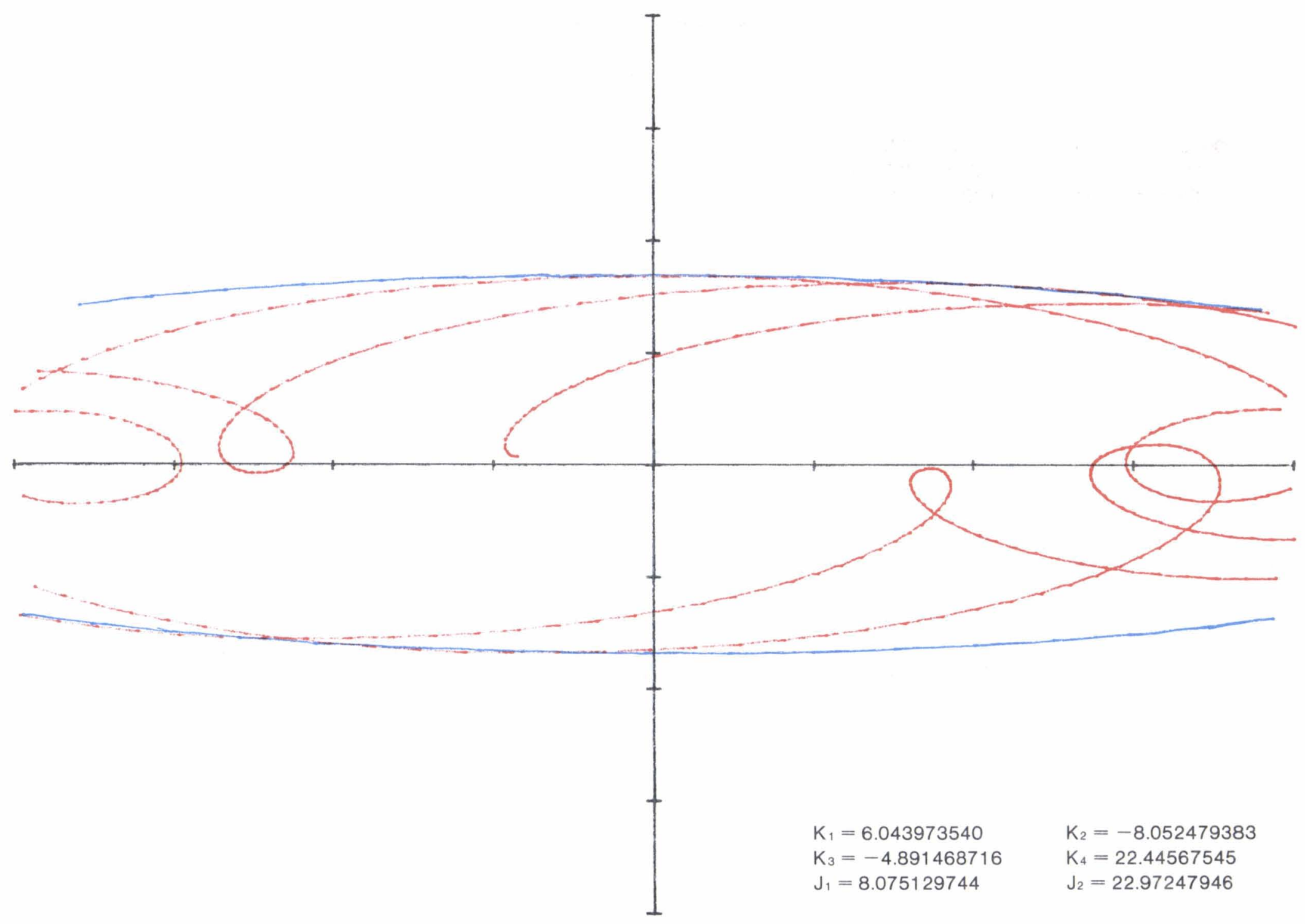

(e4) $\alpha=1, \beta=180^{\circ}, \gamma=135^{\circ}$. 


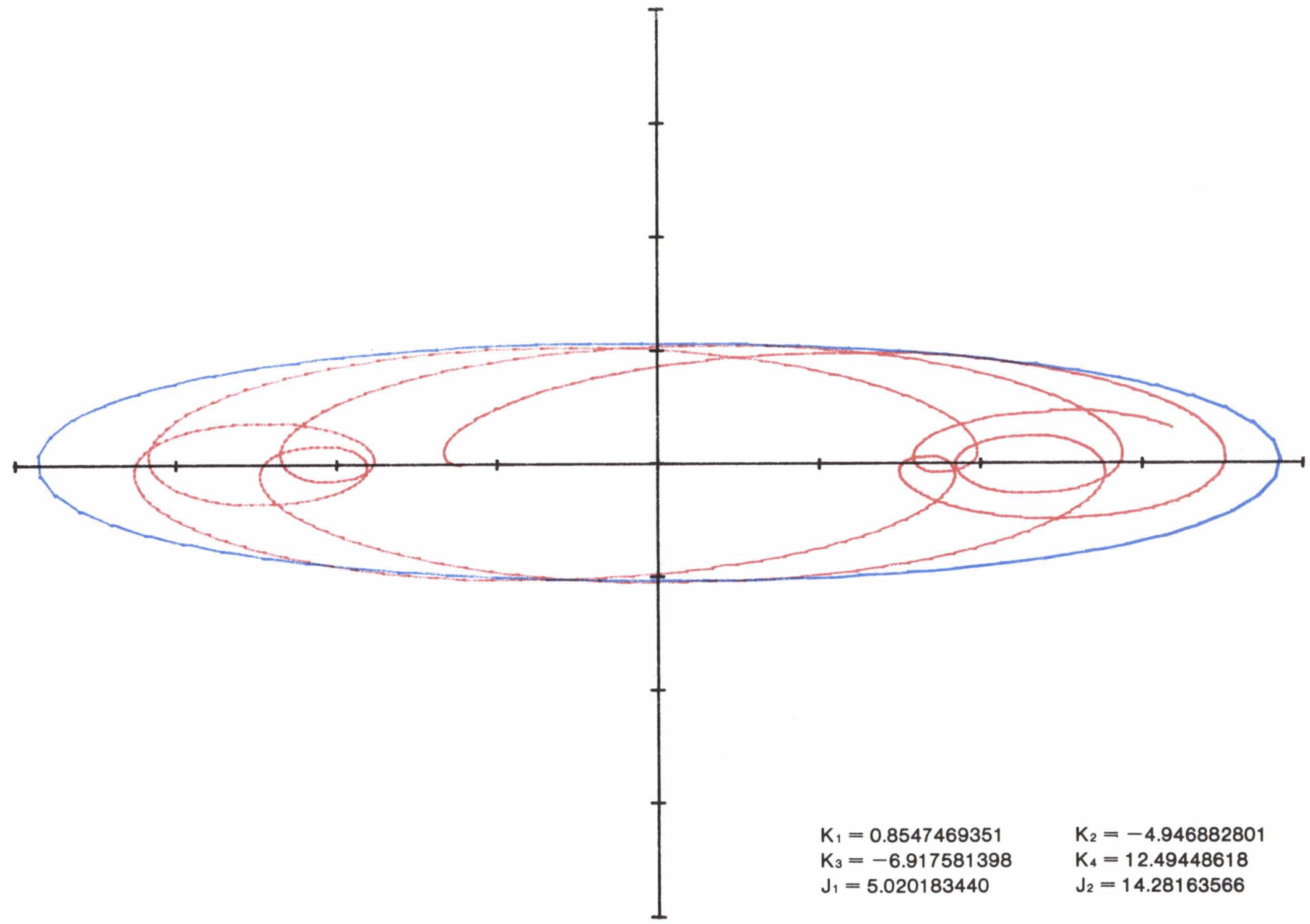

(e5) $\alpha=1, \beta=180^{\circ}, \gamma=180^{\circ}$.

FIGURE 3.-Continued. 


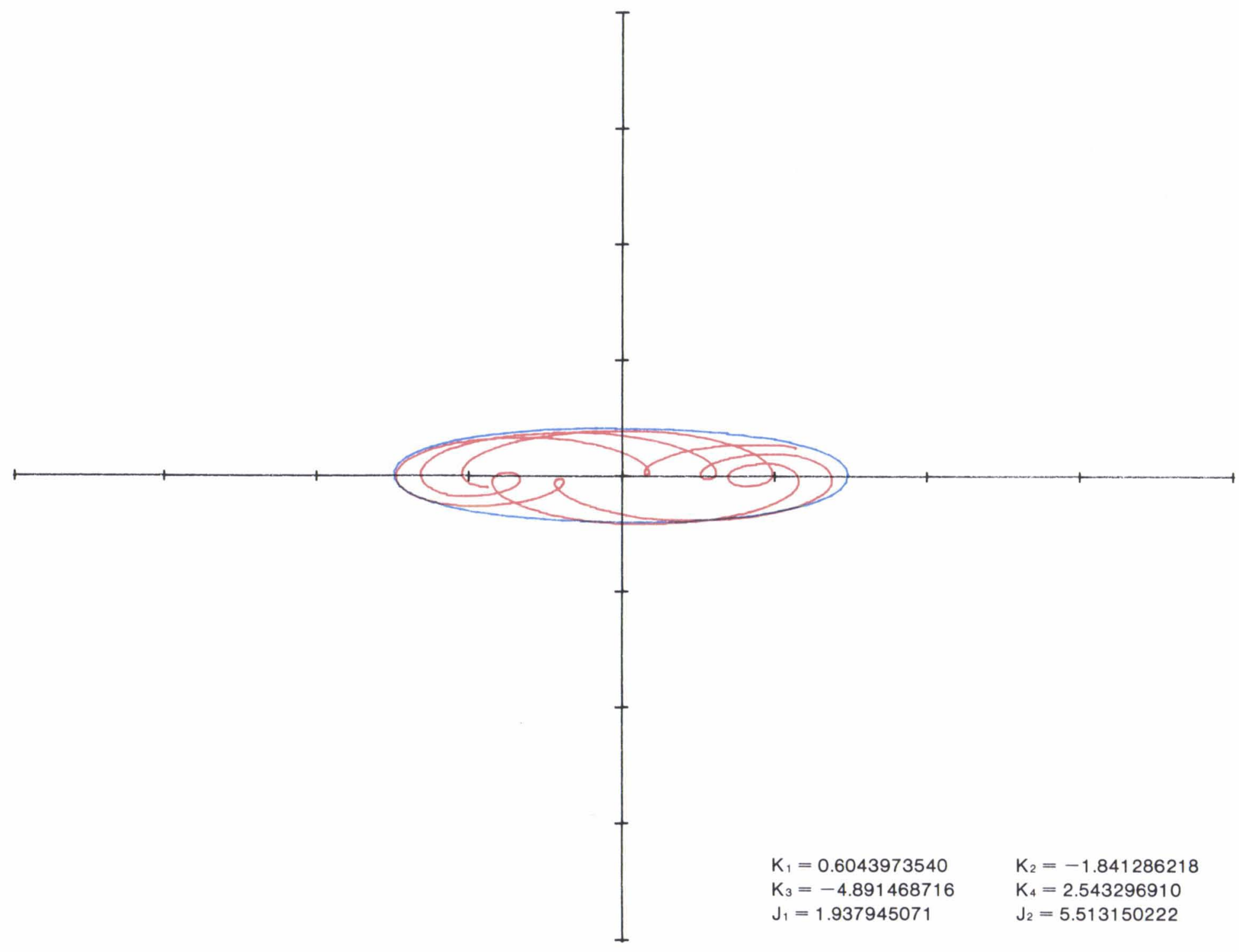

(e6) $\alpha=1, \beta=180^{\circ}, \gamma=225^{\circ}$. 


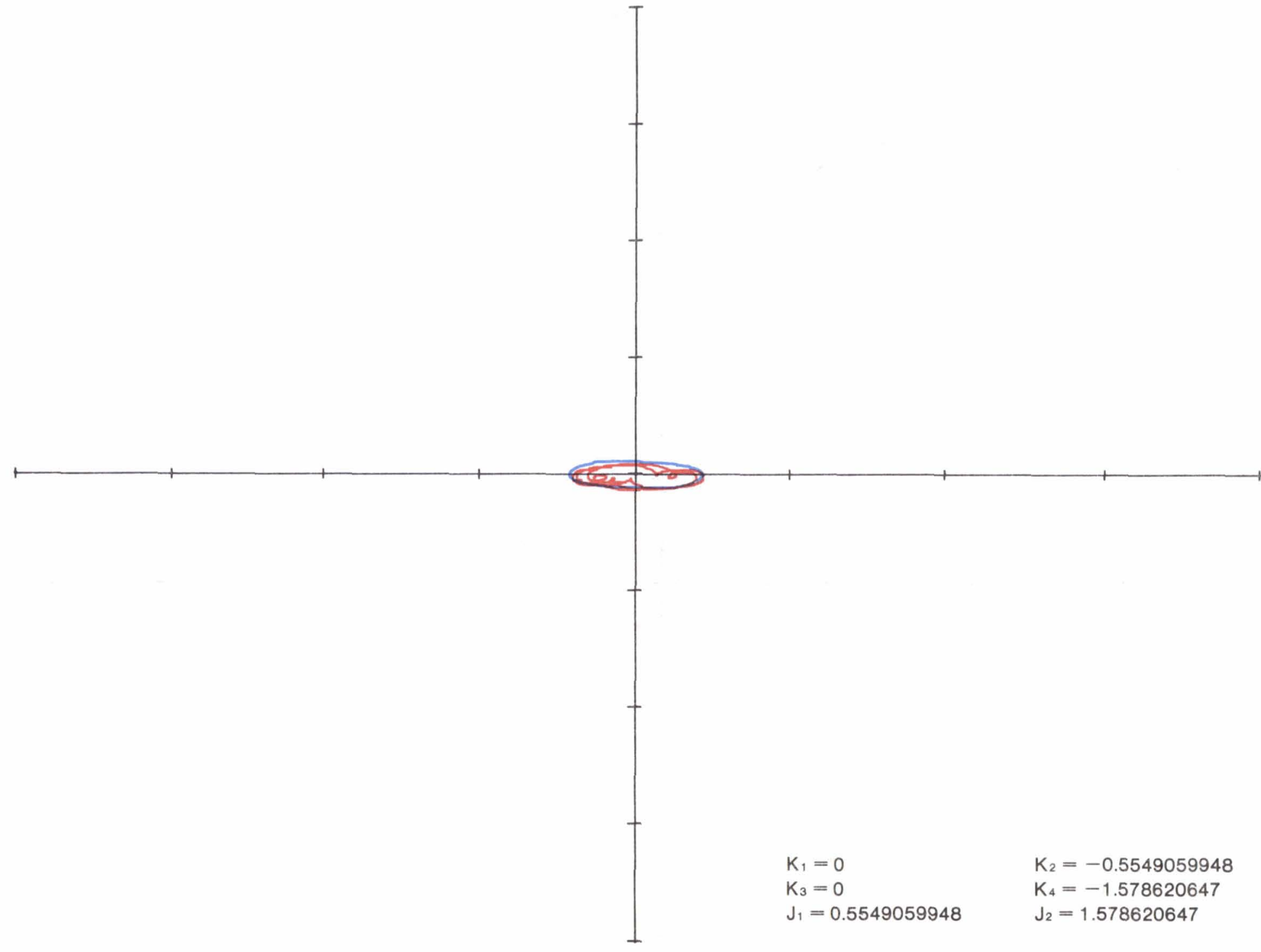

(e7) $\alpha=1, \beta=180^{\circ}, \gamma=270^{\circ}$.

FIGURE 3.-Continued. 


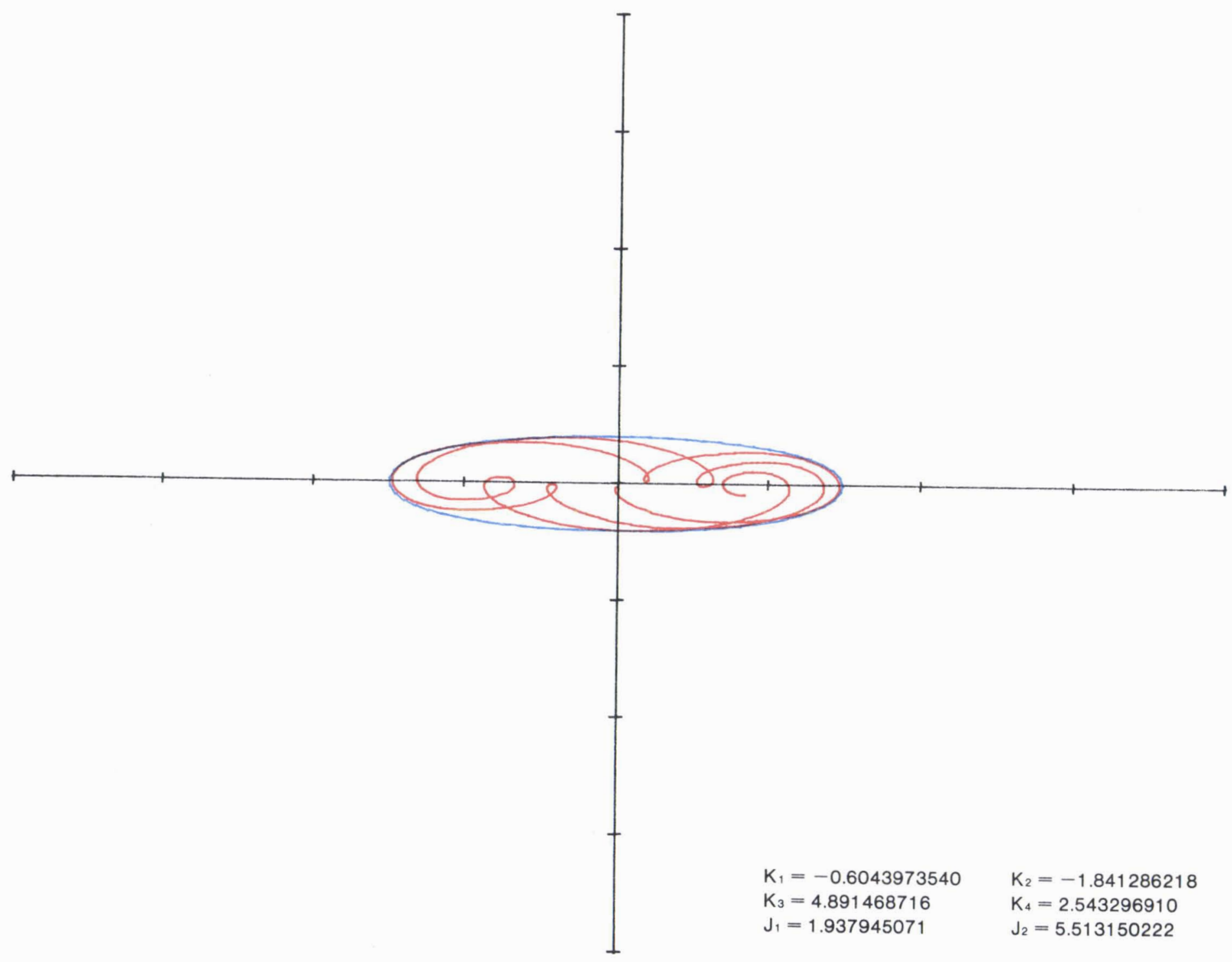

(e8) $\alpha=1, \beta=180^{\circ}, \gamma=315^{\circ}$.

FIGURE 3.-Continued. 


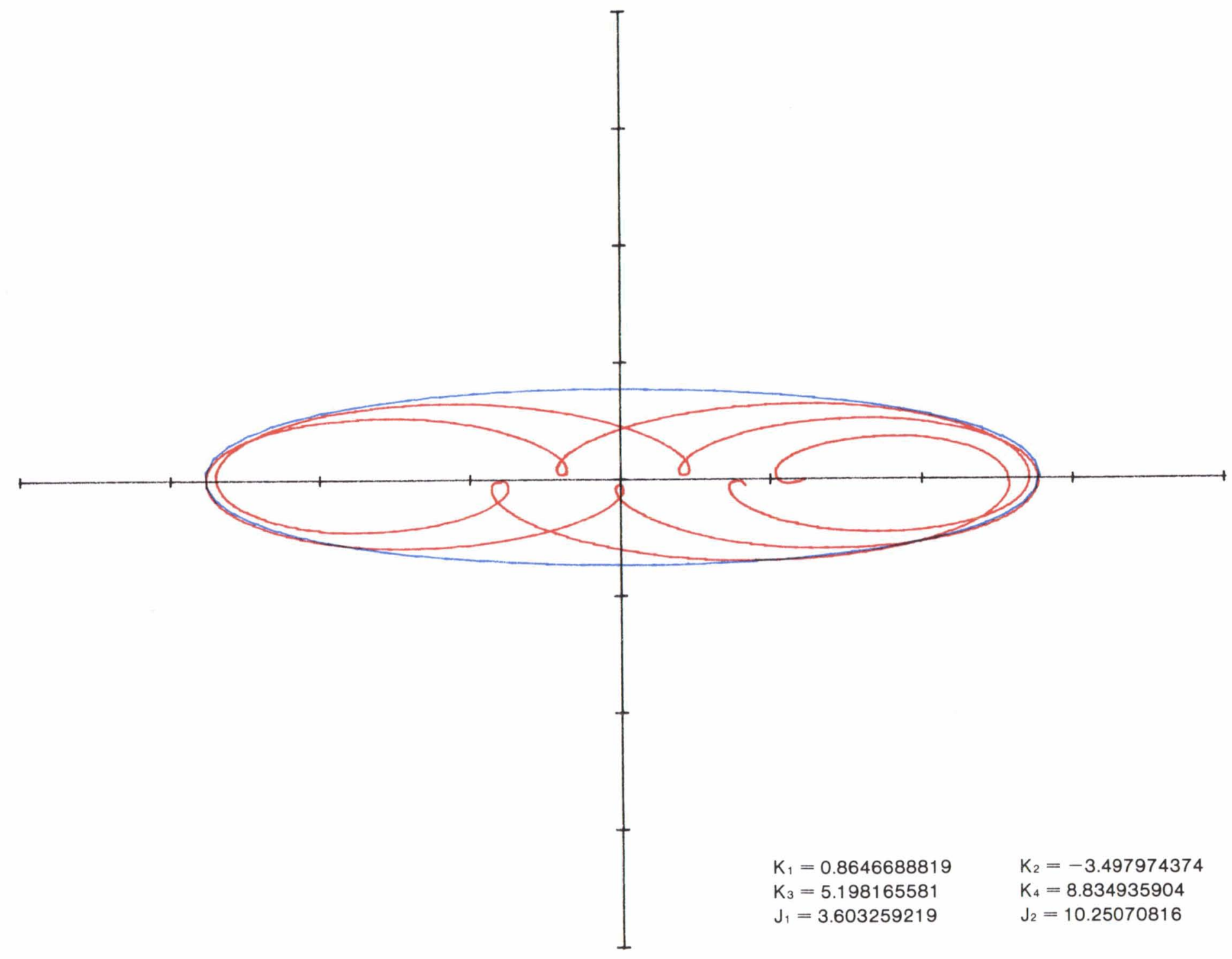

(f1) $\alpha=1, \beta=225^{\circ}, \gamma=0^{\circ}$.

FIGURE 3.-Continued. 


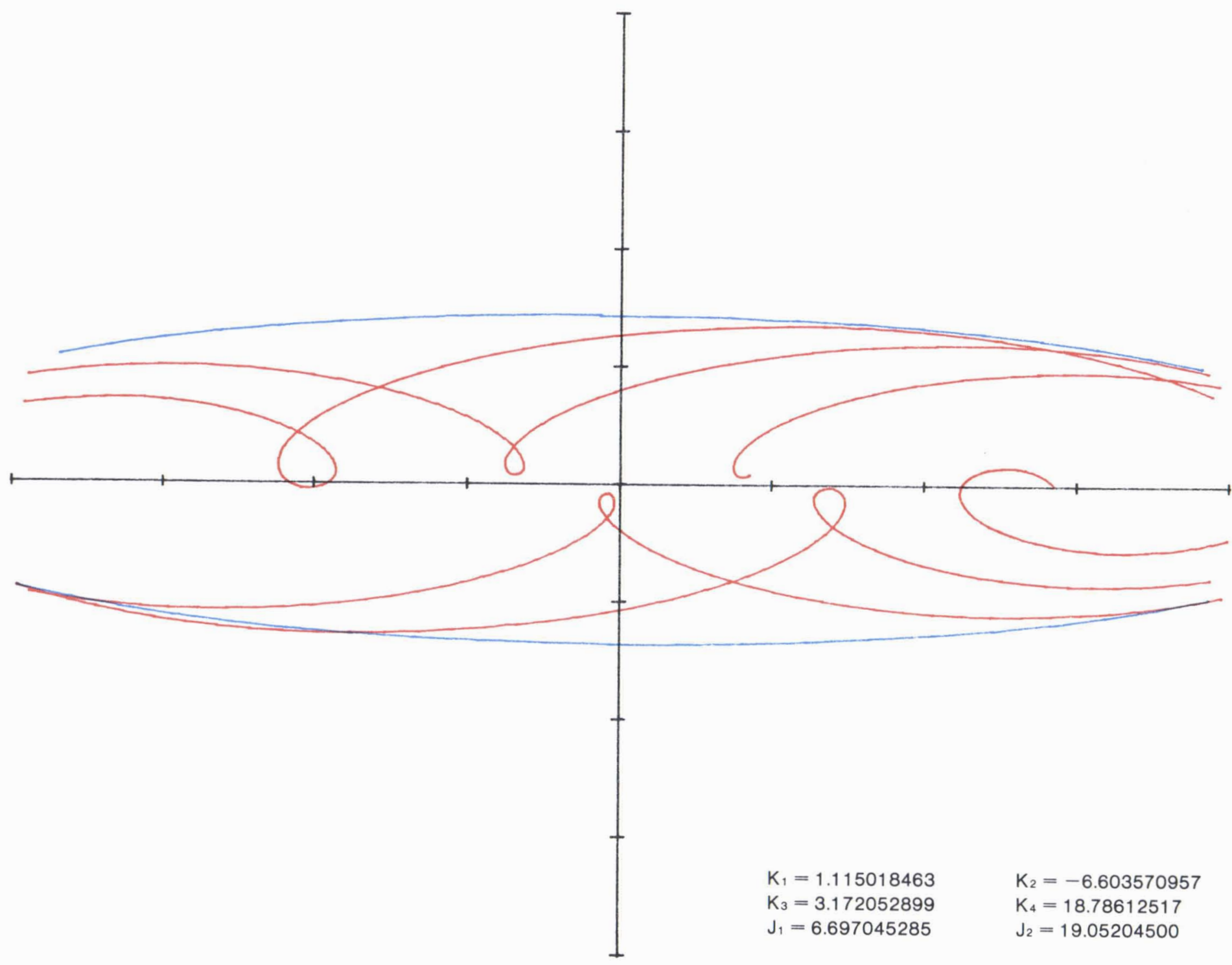

(f2) $\alpha=1, \beta=225^{\circ}, \gamma=45^{\circ}$. 


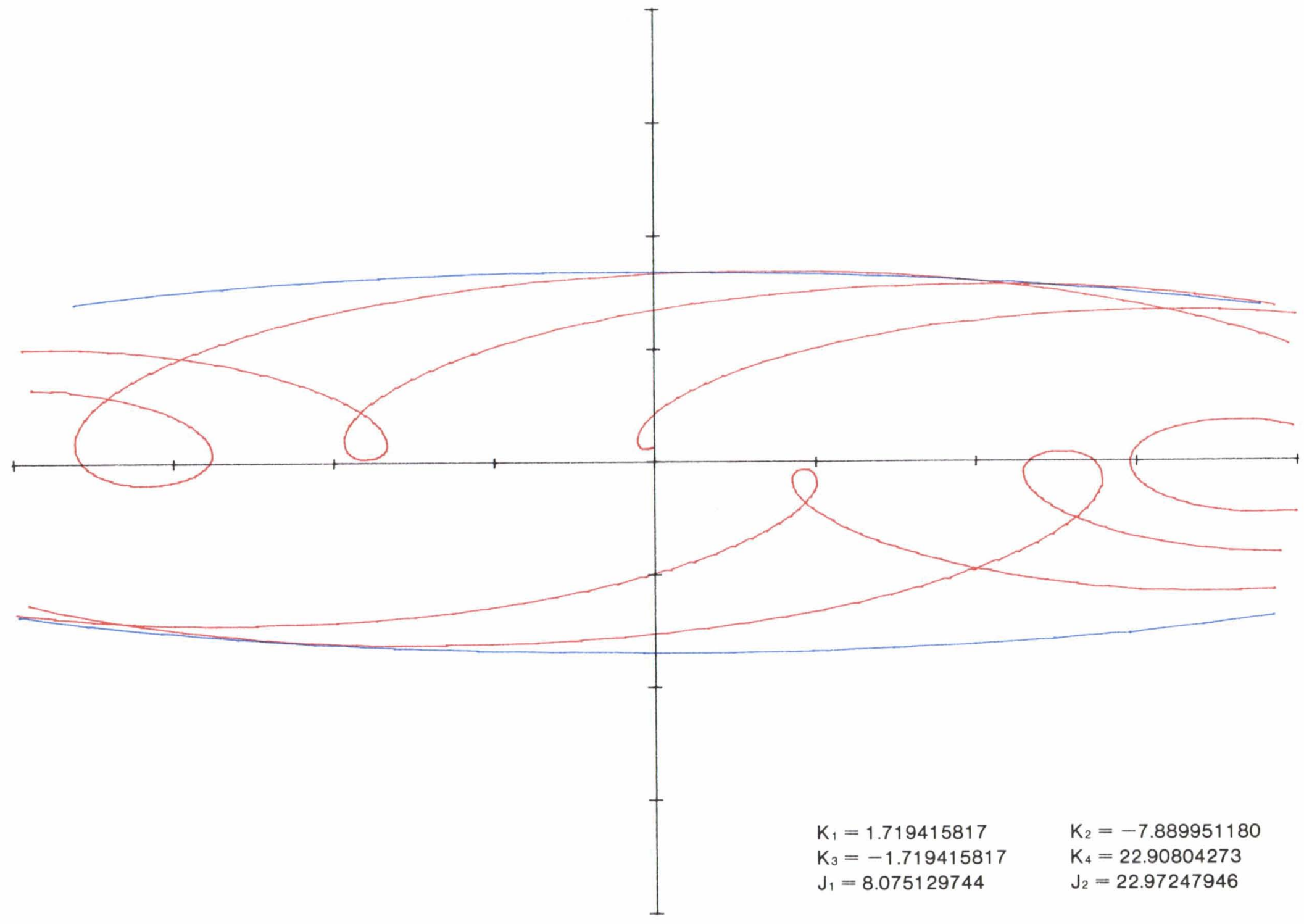

(f3) $\alpha=1, \beta=225^{\circ}, \gamma=90^{\circ}$.

FIGURE 3.-Continued. 


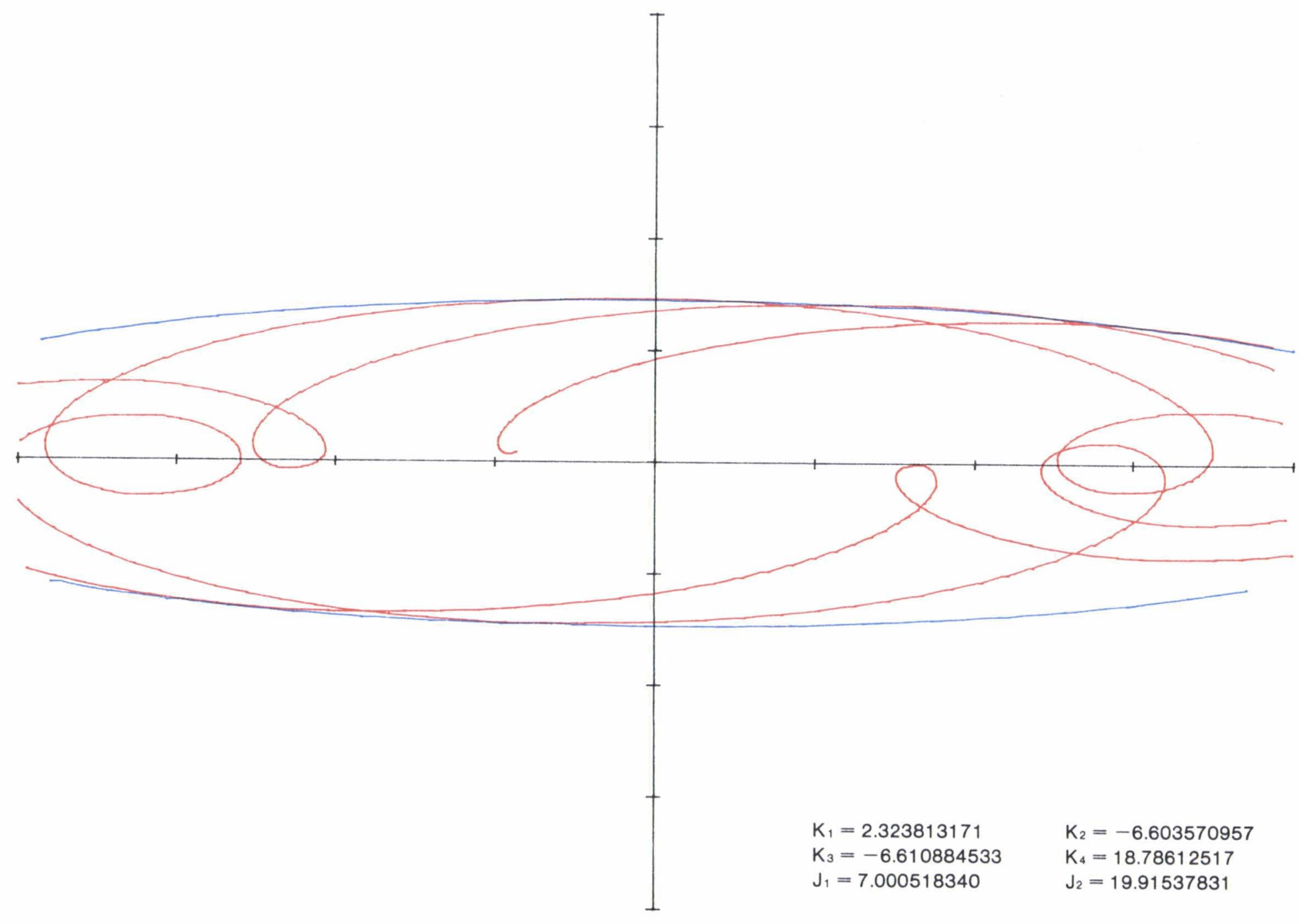

(f4) $\alpha=1, \beta=225^{\circ}, \gamma=135^{\circ}$. 


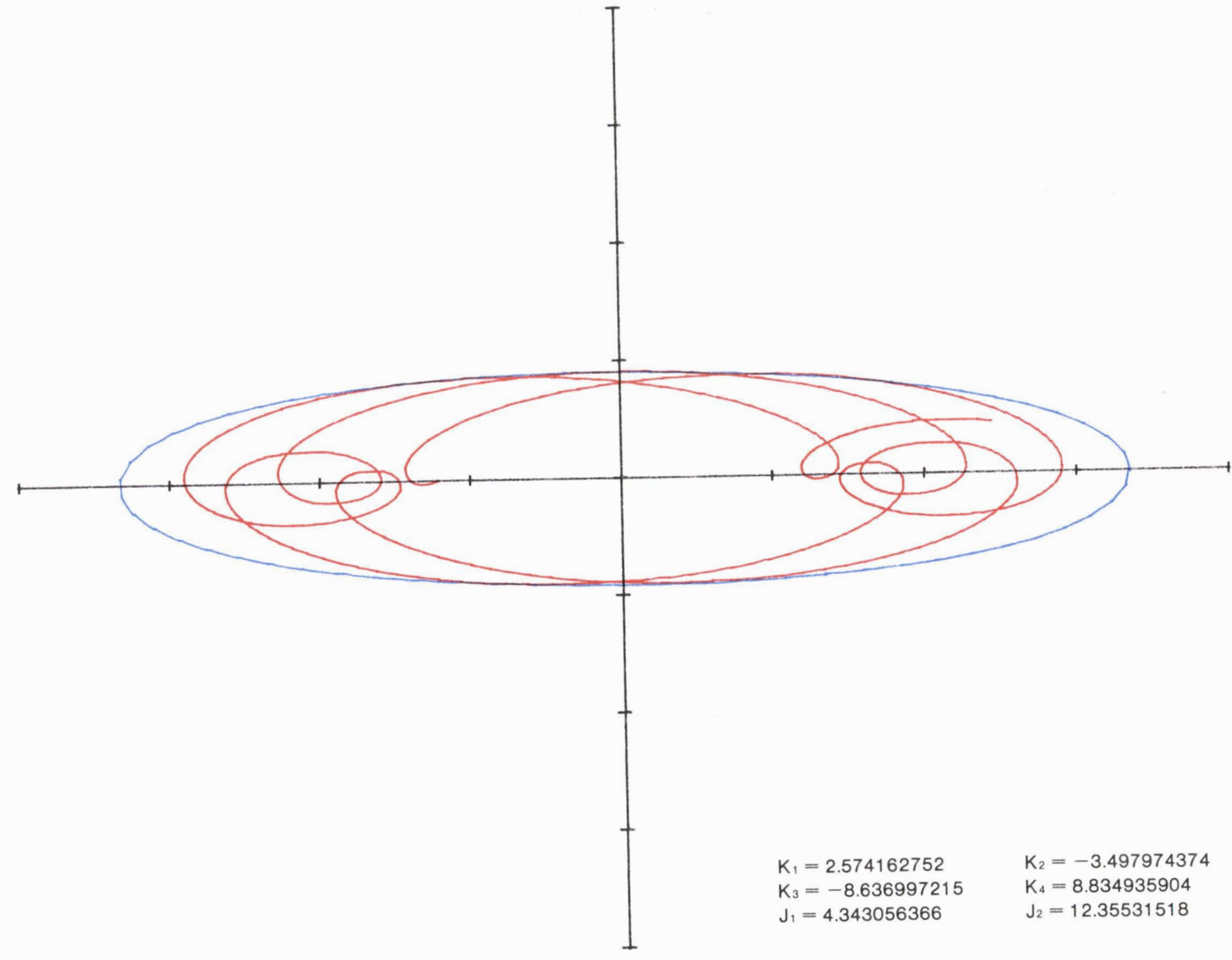

(f5) $\alpha=1, \beta=225^{\circ}, \gamma=180^{\circ}$.

FIGURE 3.-Continued. 


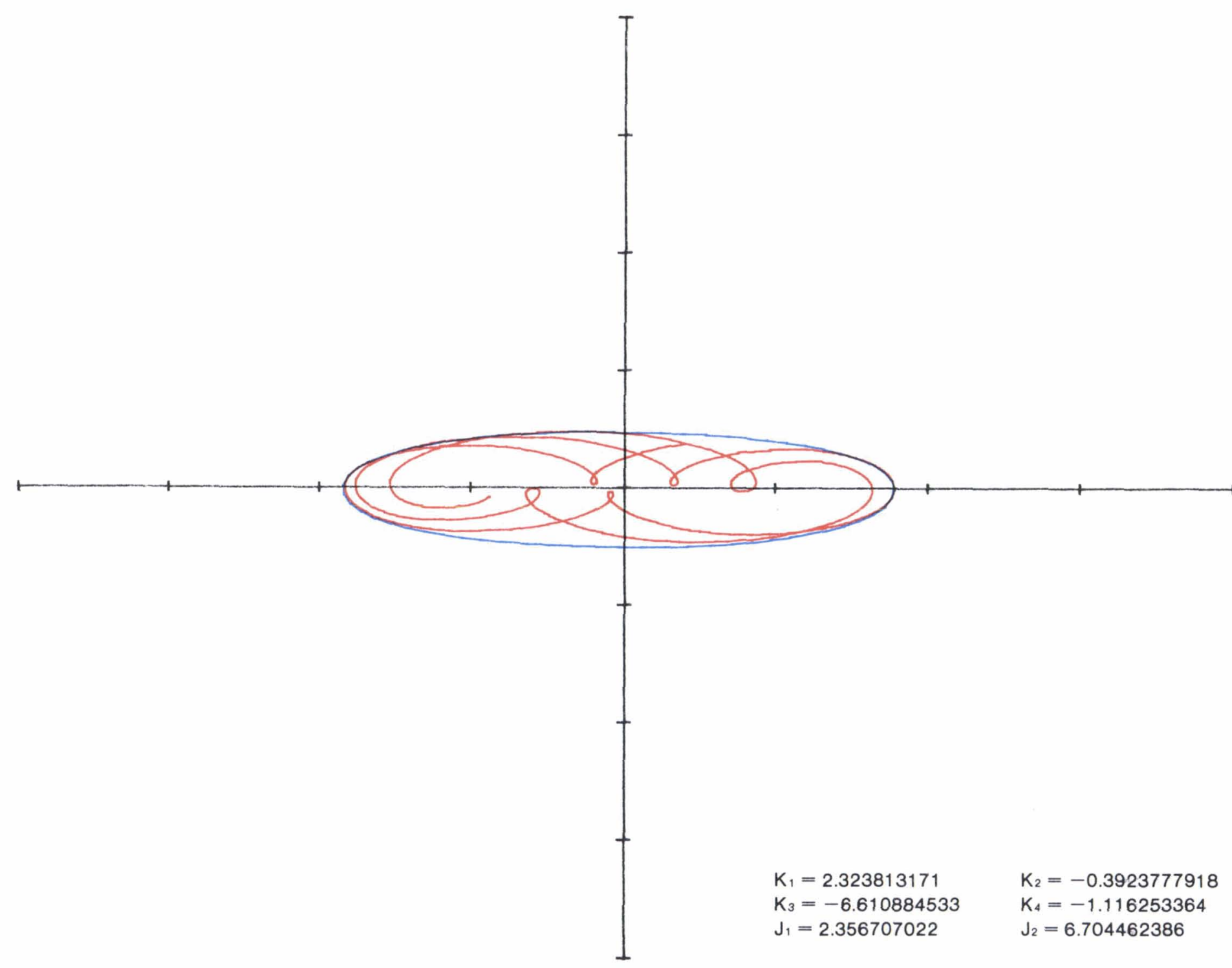

(f6) $\alpha=1, \beta=225^{\circ}, \gamma=225^{\circ}$

FIGURE 3.-Continued. 


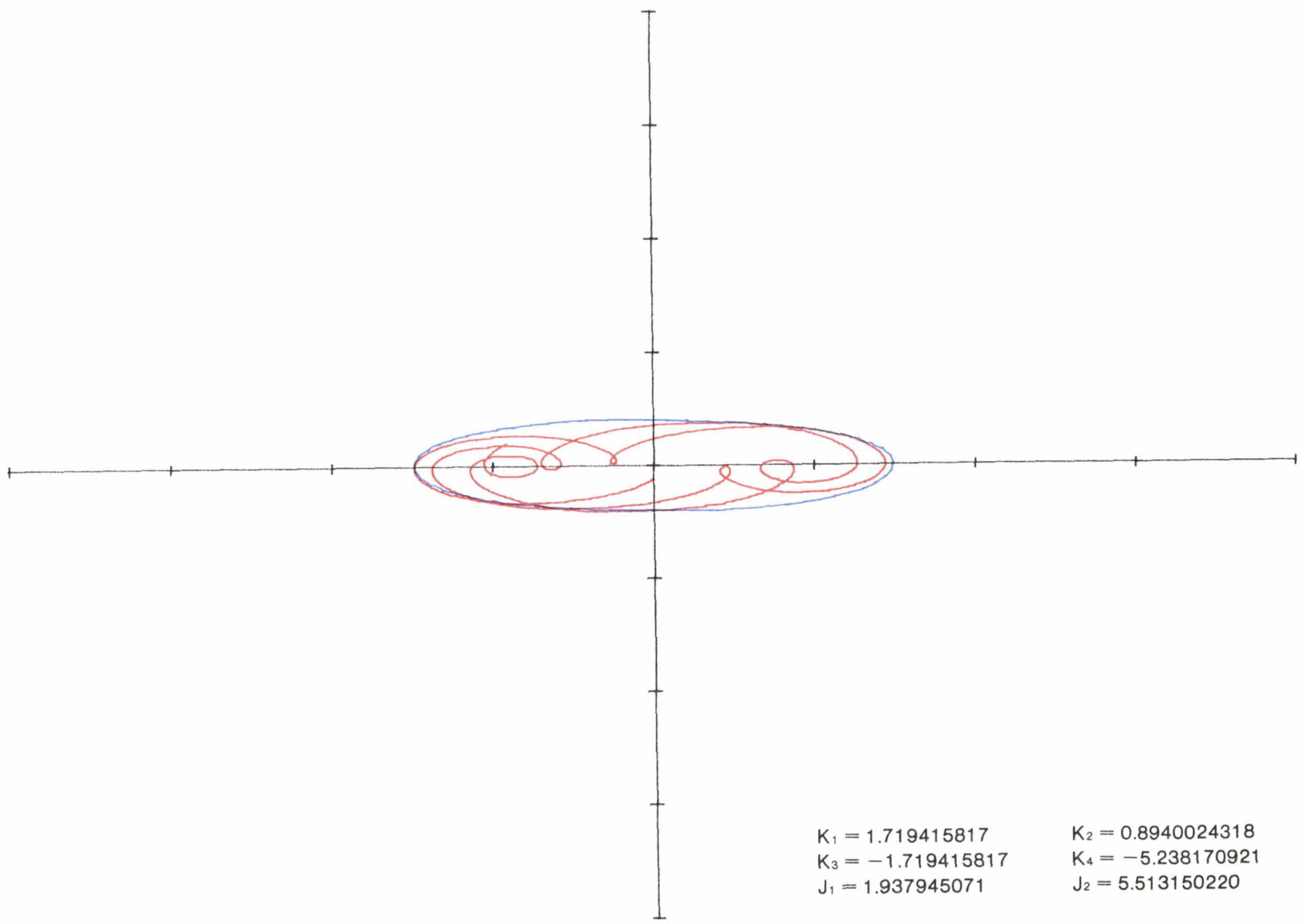

(f7) $\alpha=1, \beta=225^{\circ}, \gamma=270^{\circ}$.

FIGURE 3.-Continued. 


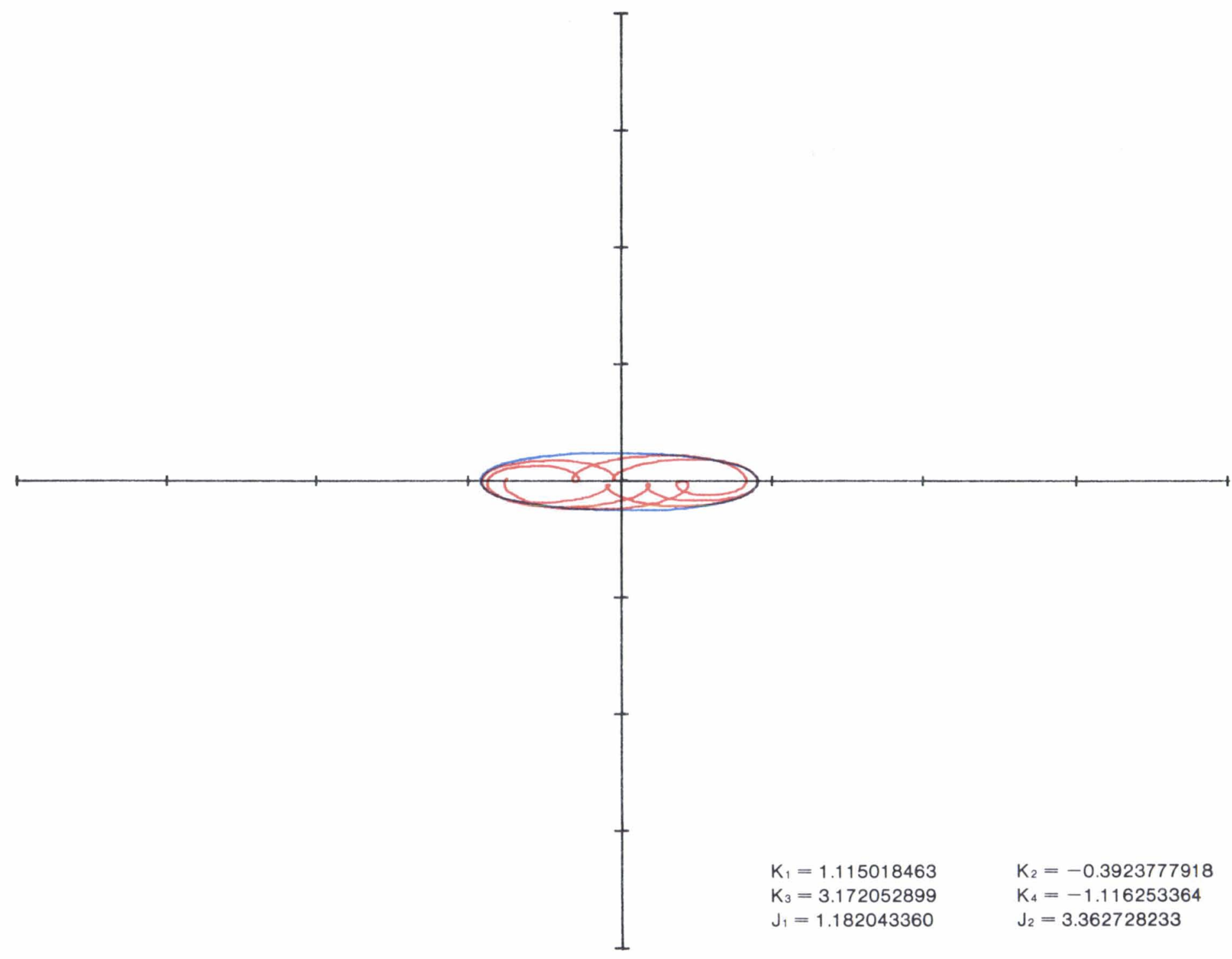

(f8) $\alpha=1, \beta=225^{\circ}, \gamma=315^{\circ}$.

FIGURE 3.-Continued. 


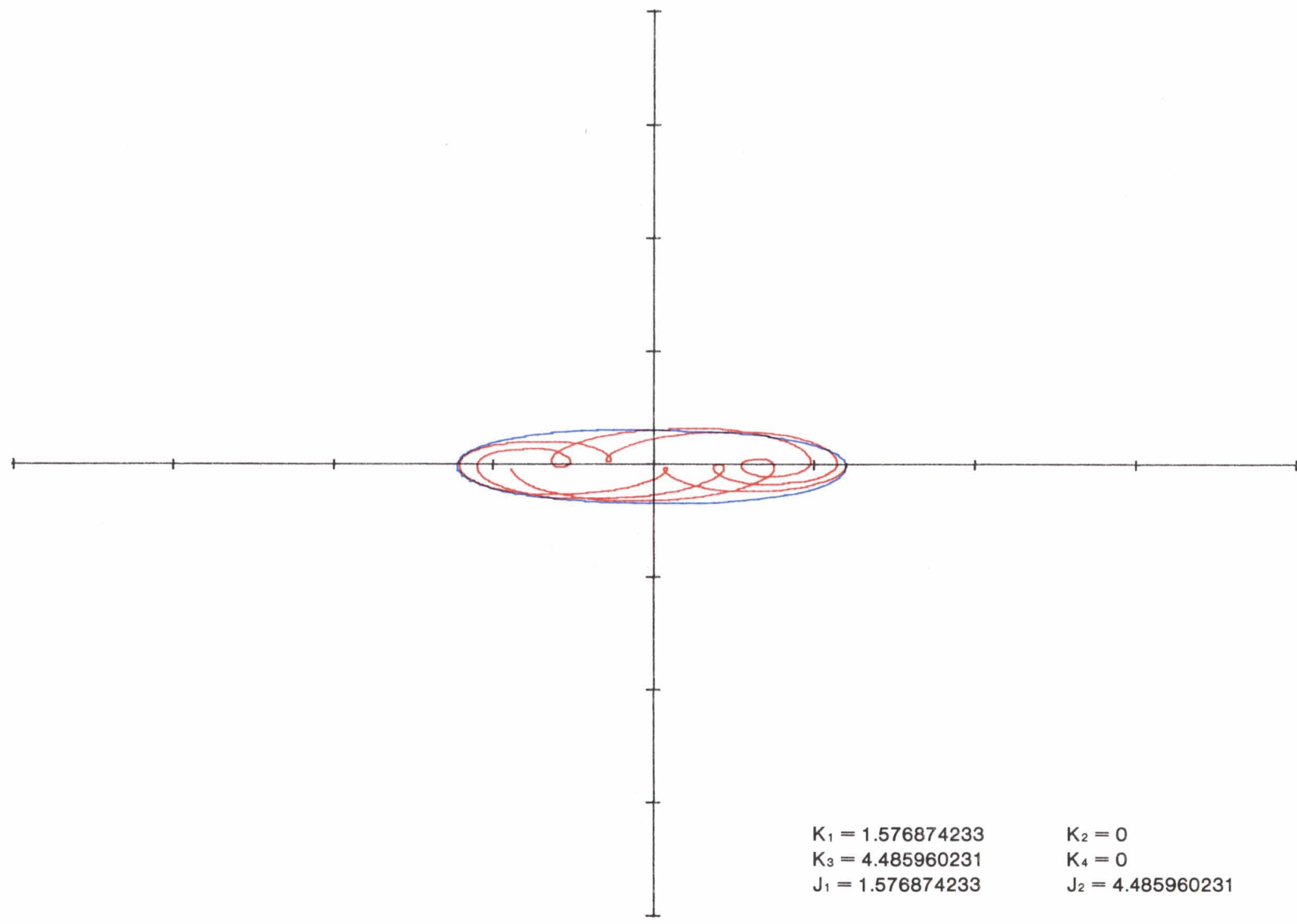

(g1) $\alpha=1, \beta=270^{\circ}, \gamma=0^{\circ}$.

FIGURE 3.-Continued. 


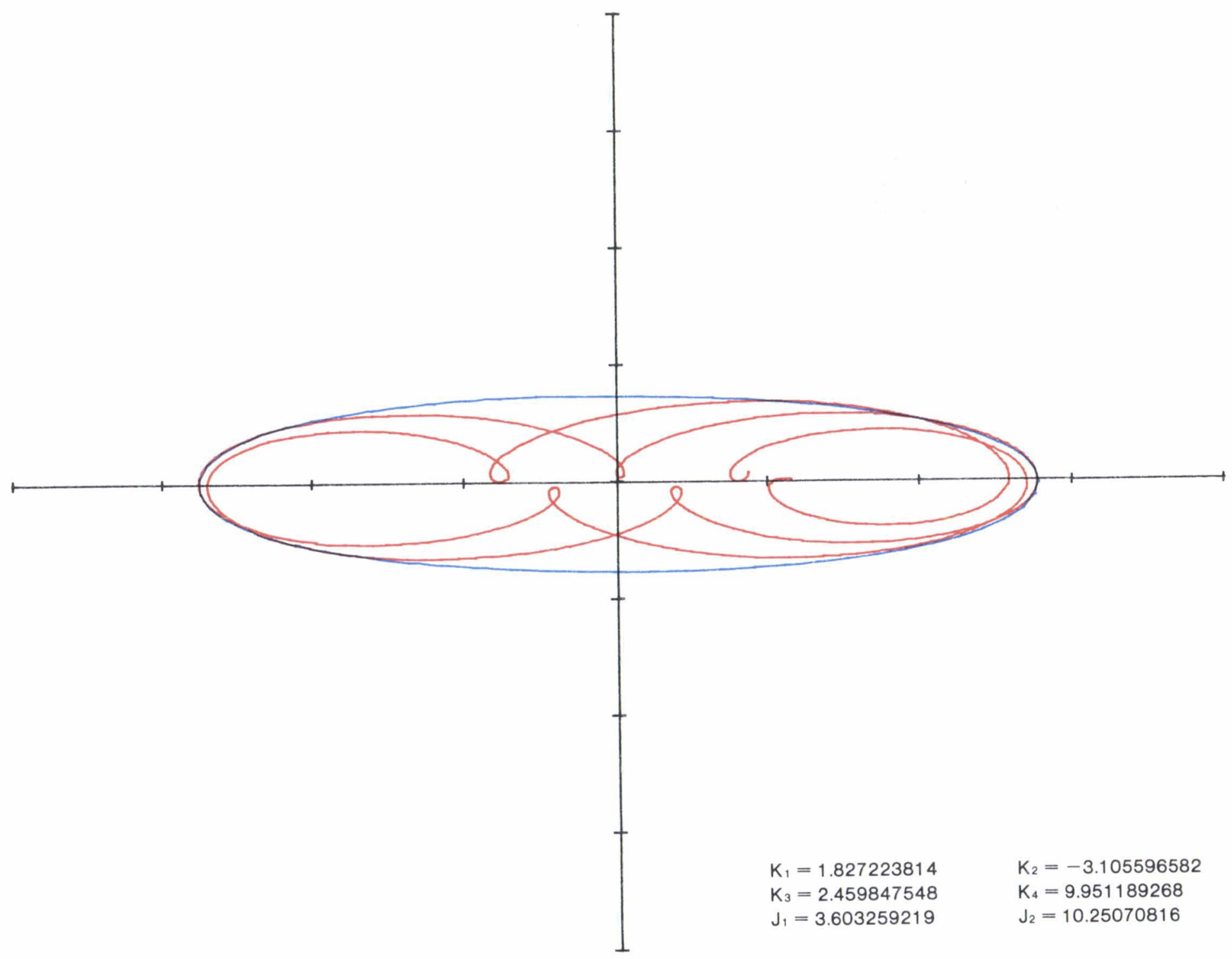

(g2) $\alpha=1, \beta=270^{\circ}, \gamma=45^{\circ}$. 


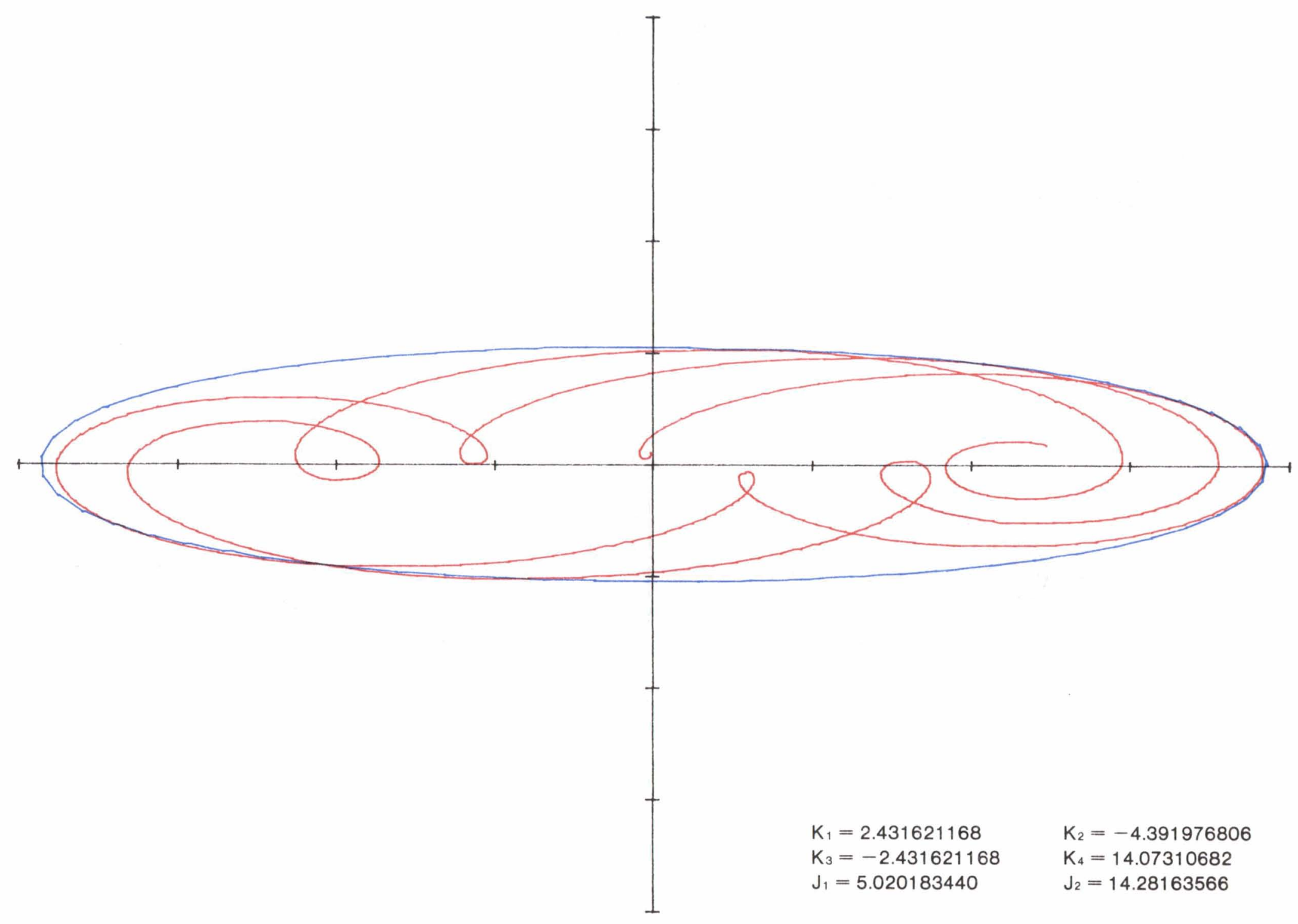

(g3) $\alpha=1, \beta=270^{\circ}, \gamma=90^{\circ}$.

FIGURE 3.-Continued. 


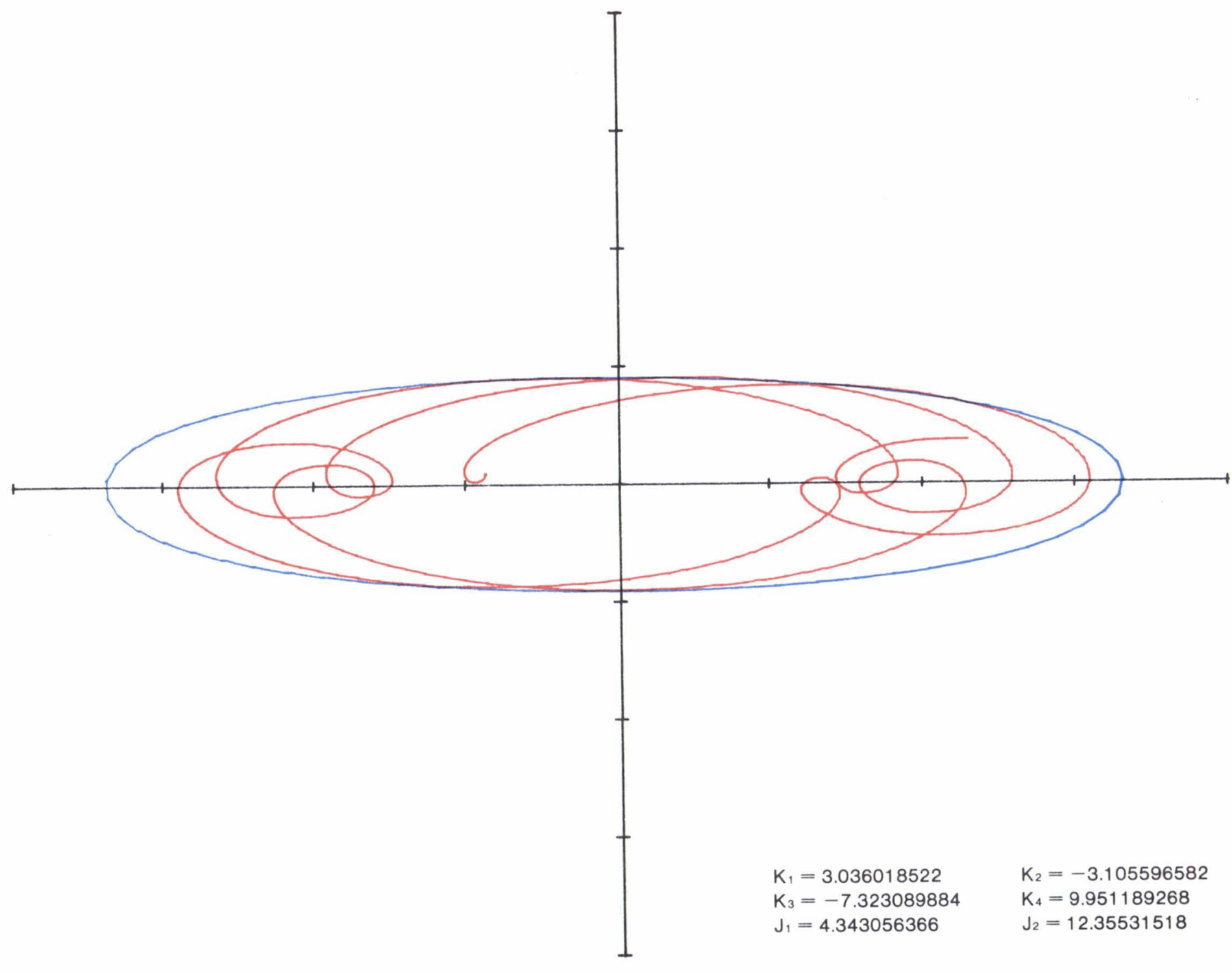

(g4) $\alpha=1, \beta=270^{\circ}, \gamma=135^{\circ}$.

FIGURE 3.-Continued. 


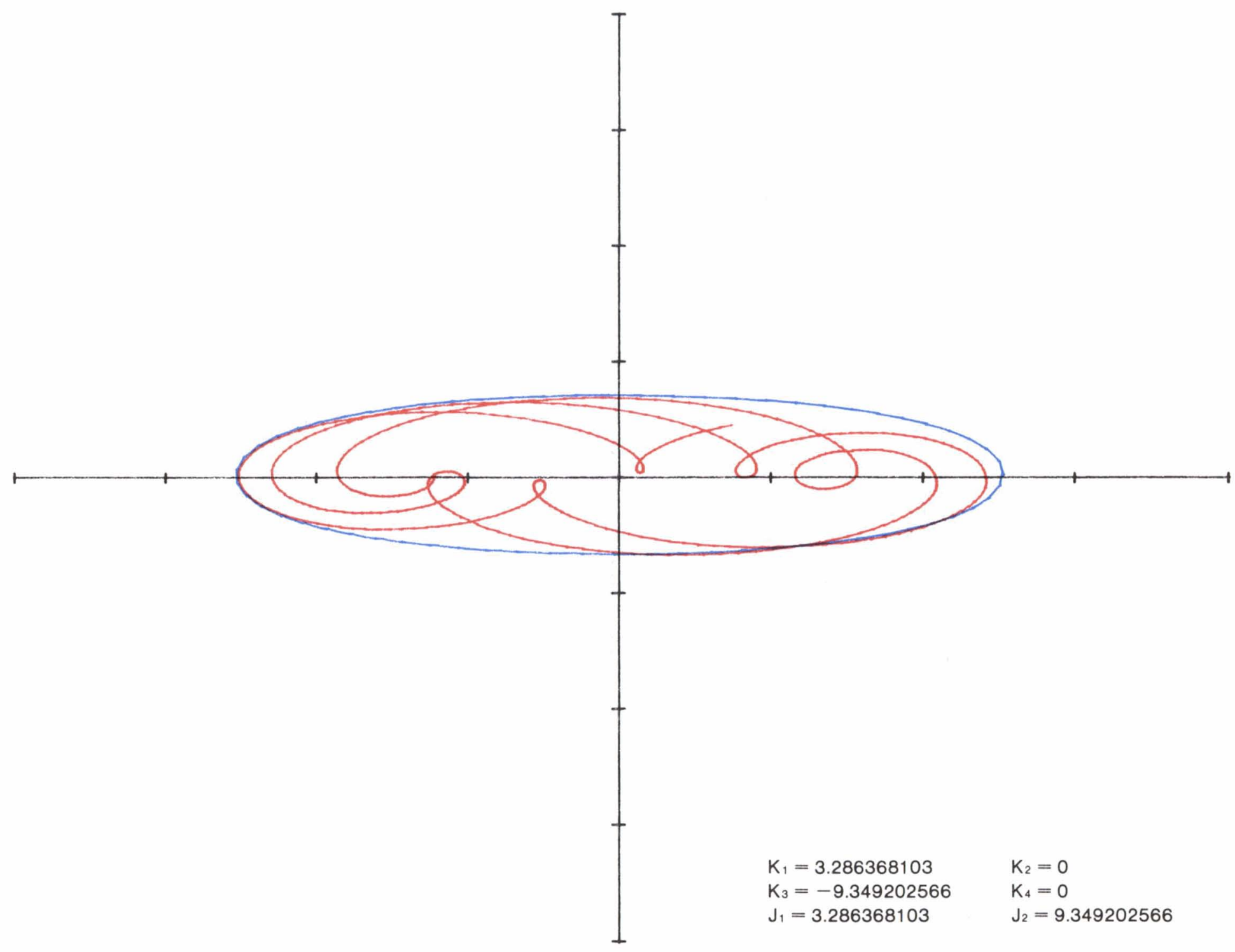

(g5) $\alpha=1, \beta=270^{\circ}, \gamma=180^{\circ}$.

FIGURE 3.-Continued. 


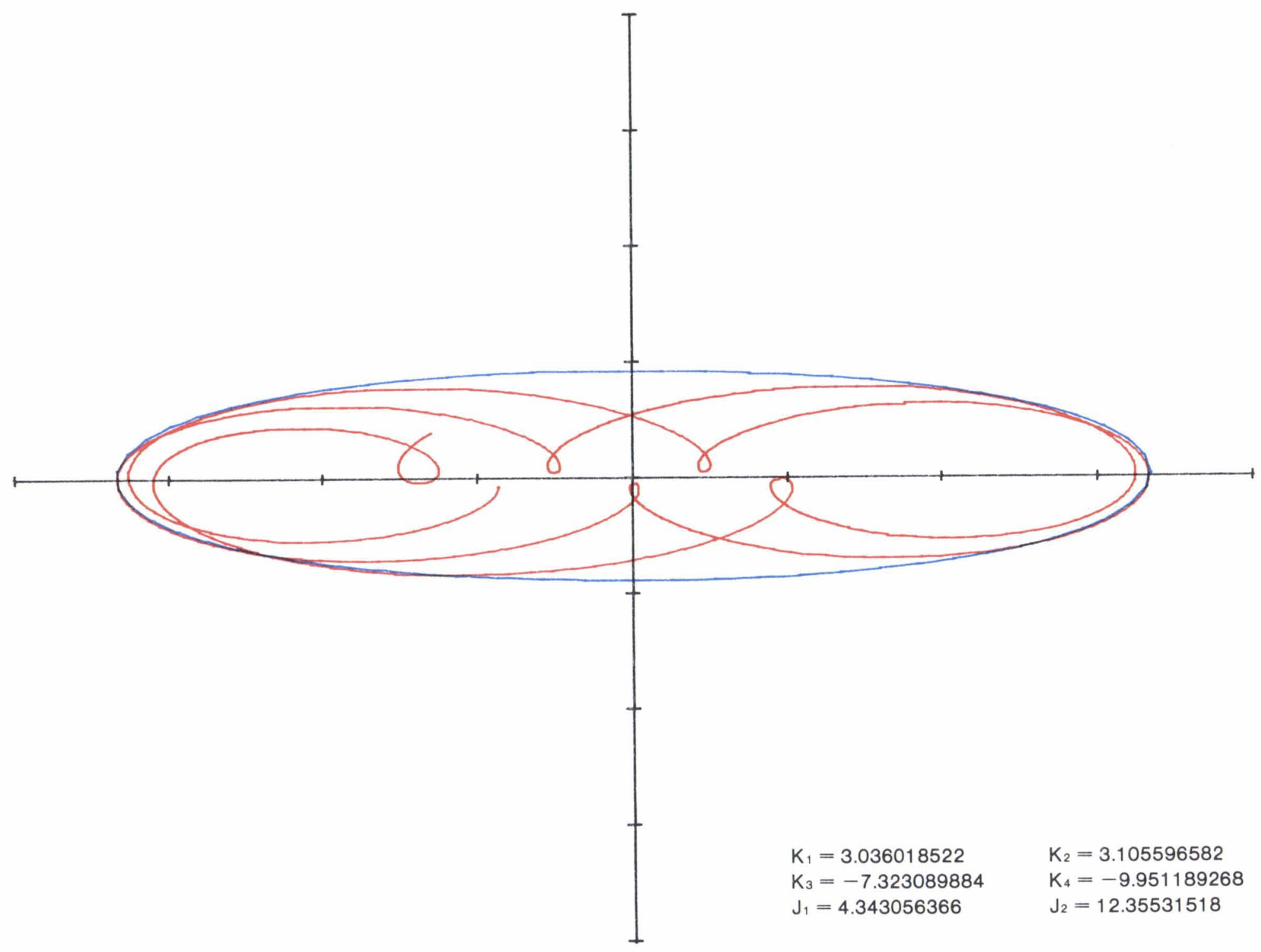

(g6) $\alpha=1, \beta=270^{\circ}, \gamma=225^{\circ}$. 


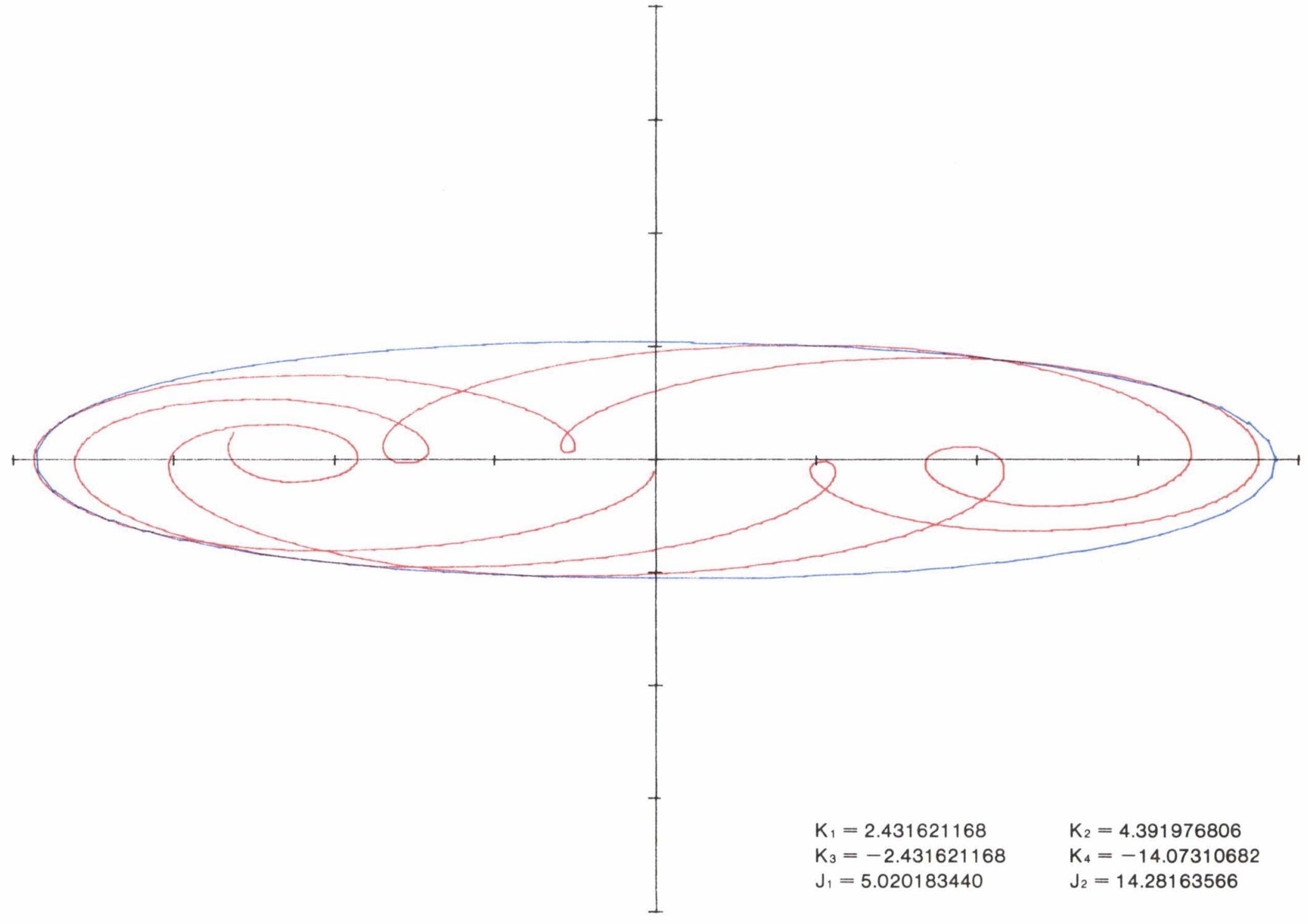

(g7) $\alpha=1, \beta=270^{\circ}, \gamma=270^{\circ}$.

FIGURE 3.-Continued. 


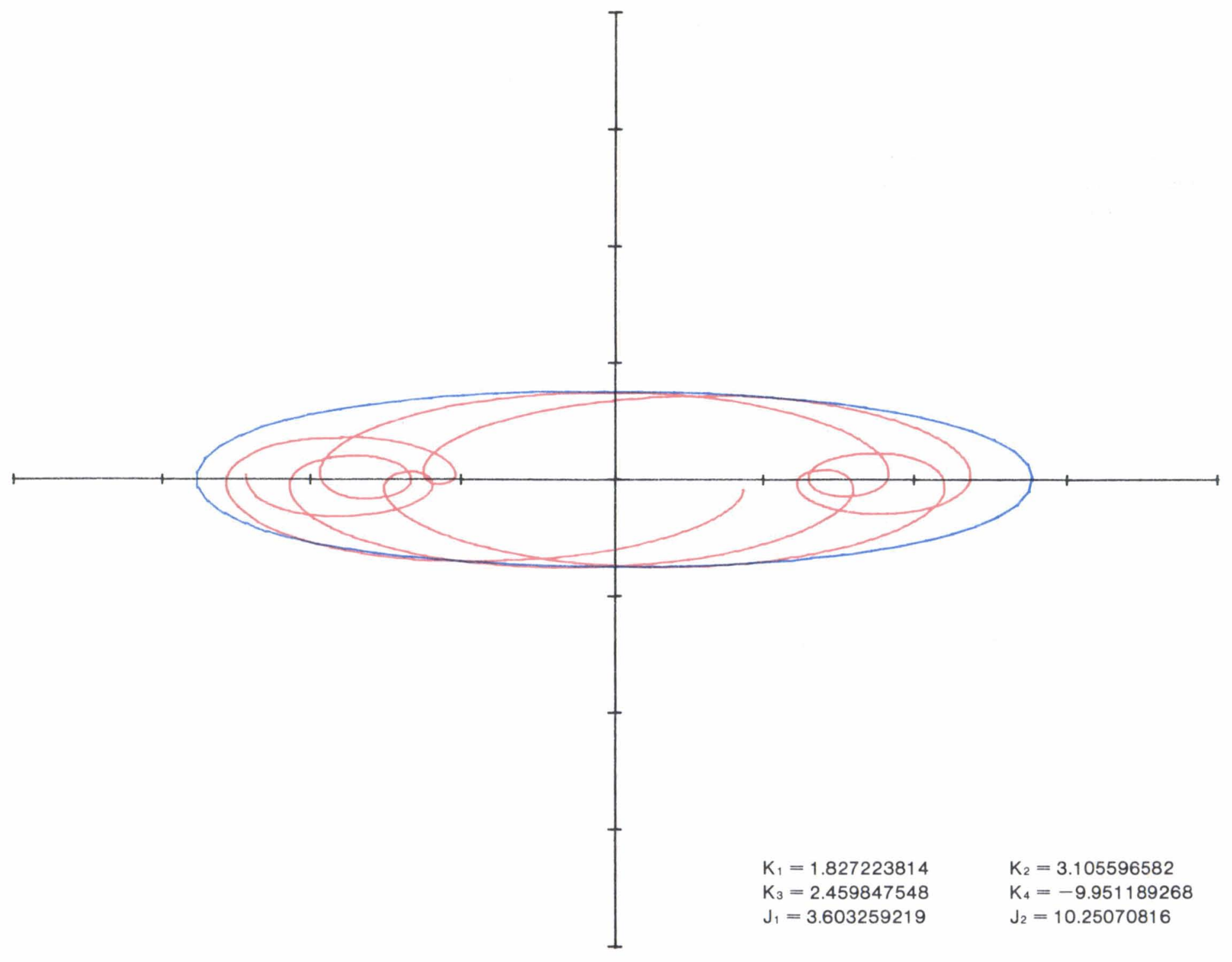

(g8) $\alpha=1, \beta=270^{\circ}, \gamma=315^{\circ}$.

FIGURE 3.-Continued. 


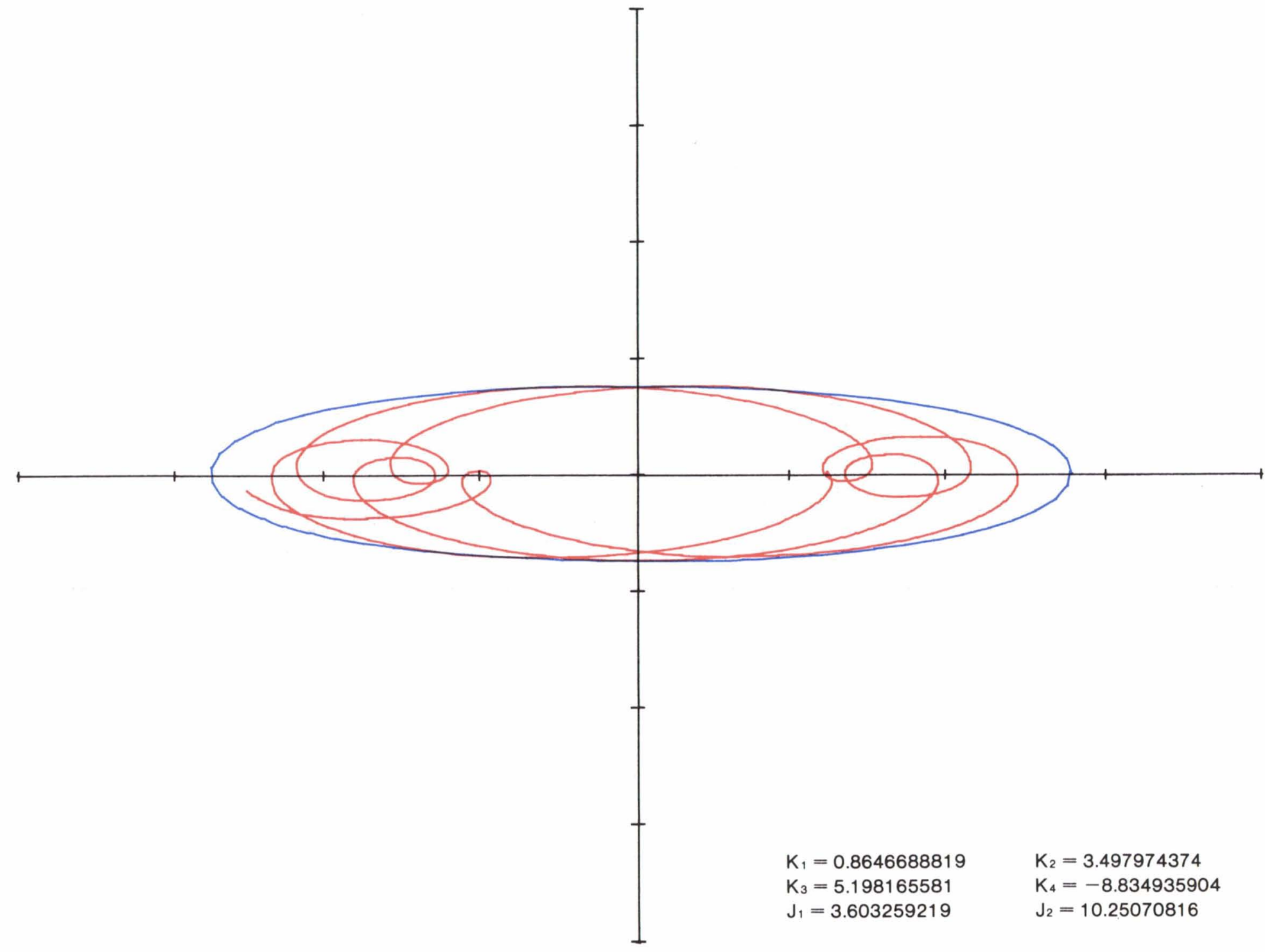

(h1) $\alpha=1, \beta=315^{\circ}, \gamma=0^{\circ}$.

FIGURE 3.-Continued. 


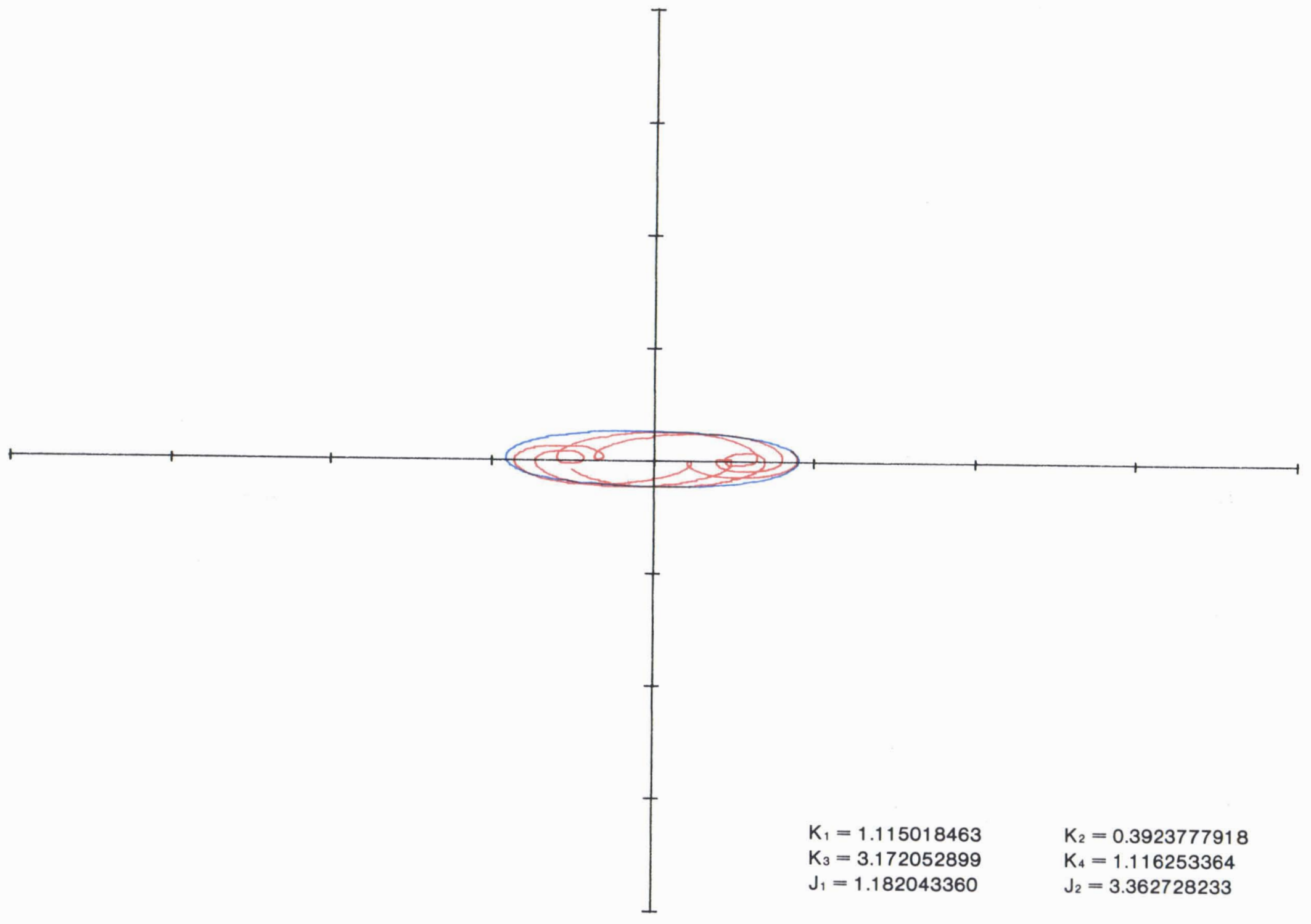

(h2) $\alpha=1, \beta=315^{\circ}, \gamma=45^{\circ}$.

FIGURE 3.-Continued. 


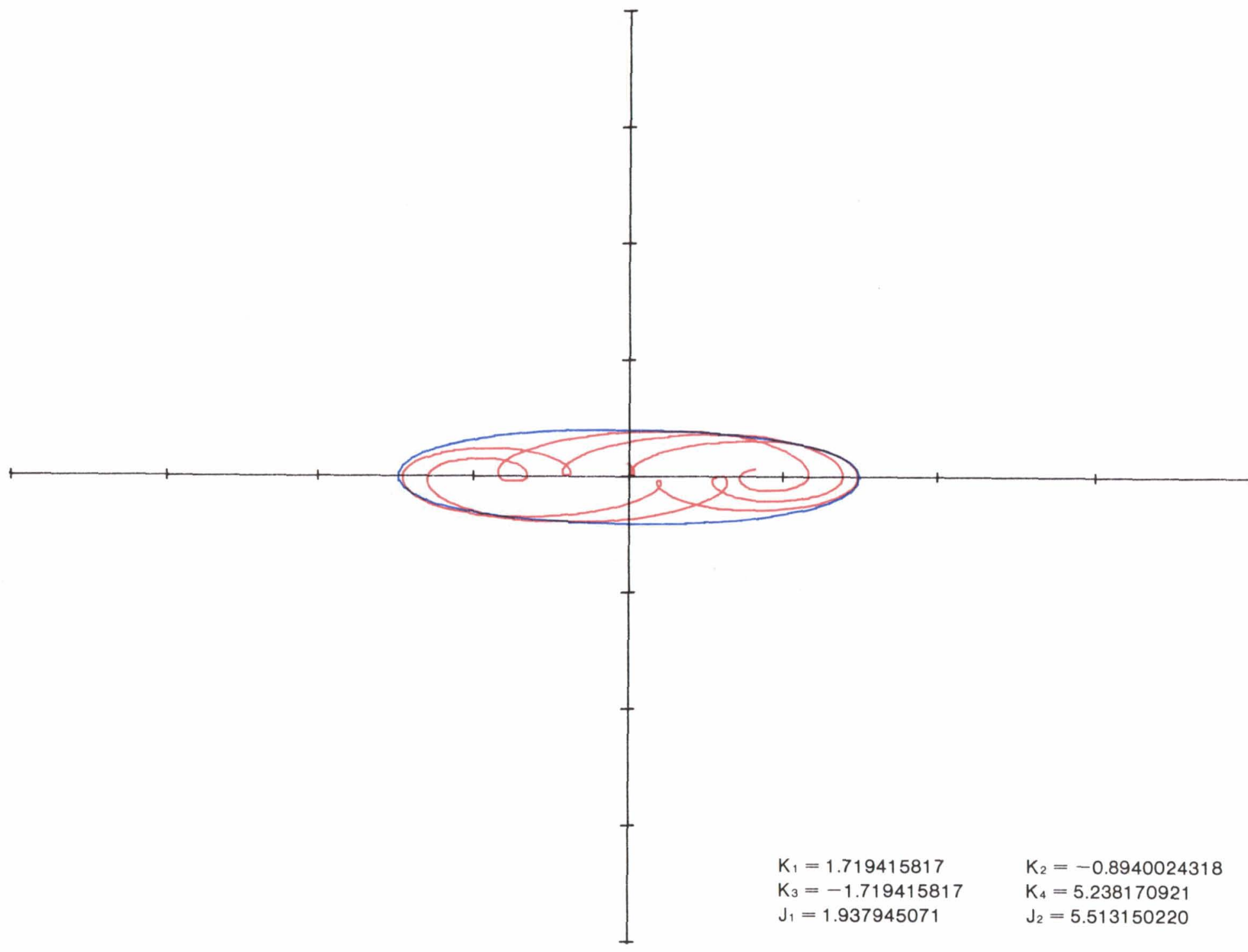

(h3) $\alpha=1, \beta=315^{\circ}, \gamma=90^{\circ}$.

FIGURE 3.-Continued. 


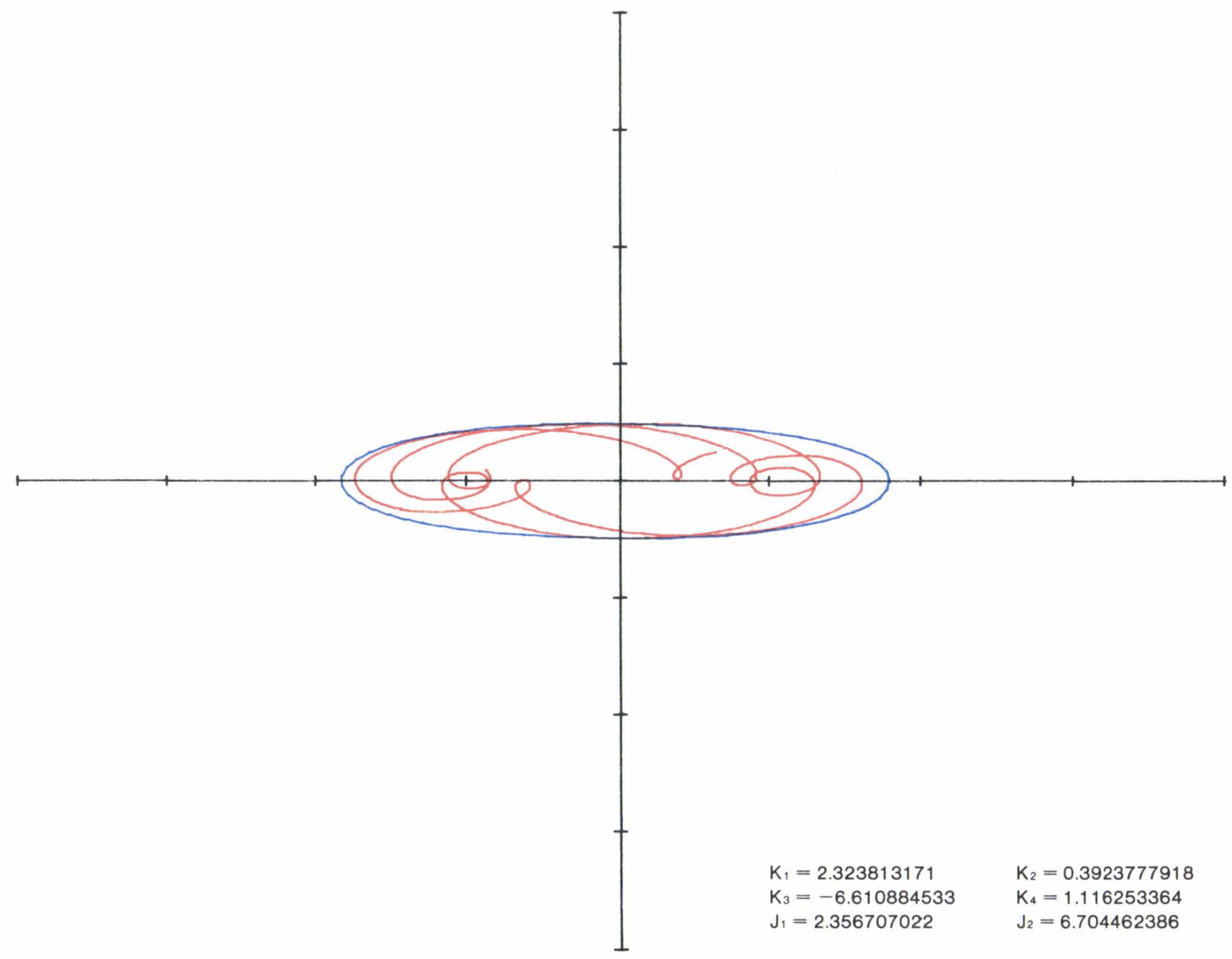

(h4) $\alpha=1, \beta=315^{\circ}, \gamma=135^{\circ}$.

FIGURE 3.-Continued. 


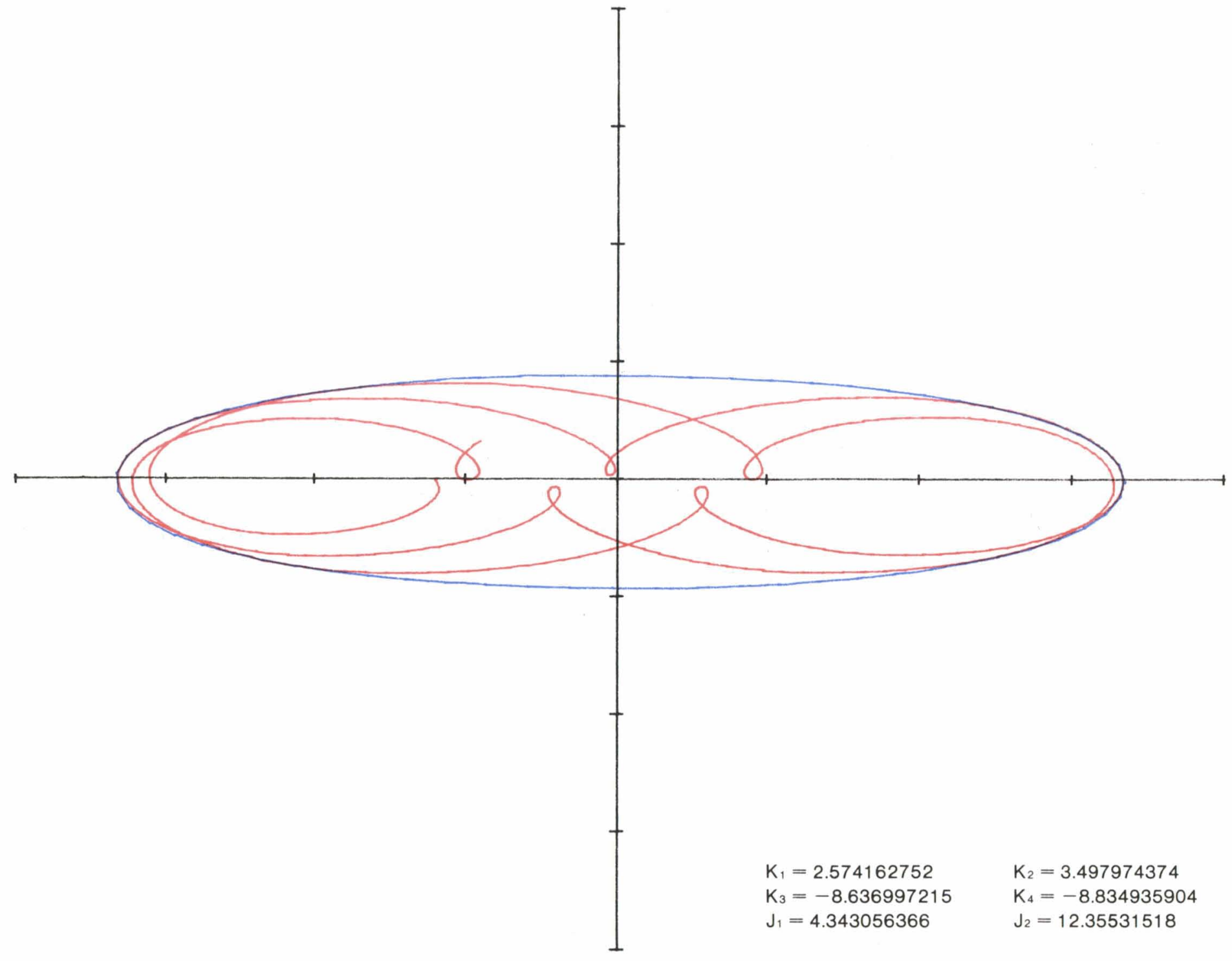

(h5) $\alpha=1, \beta=315^{\circ}, \gamma=180^{\circ}$.

FIGURE 3.-Continued. 


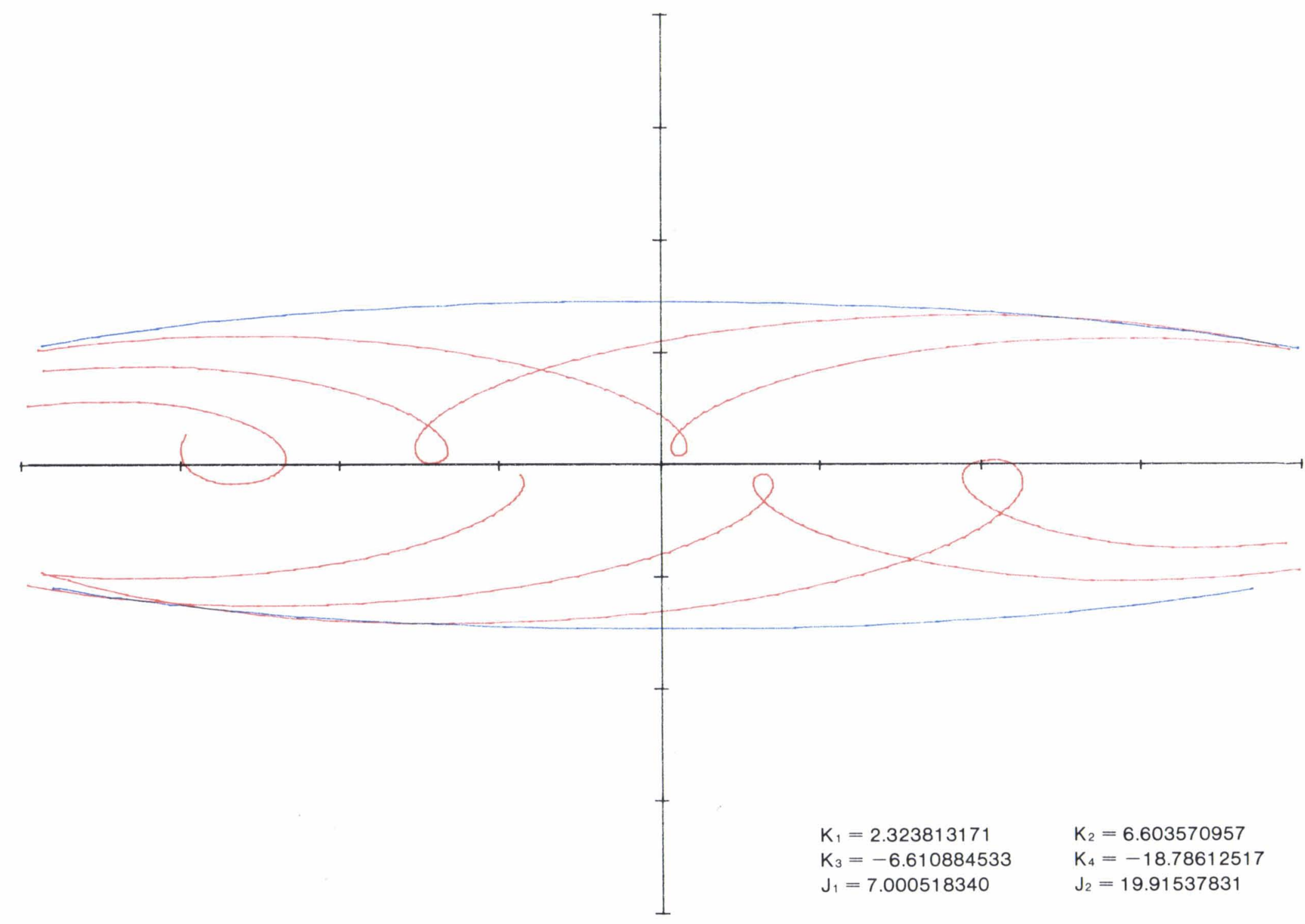

(h6) $\alpha=1, \beta=315^{\circ}, \gamma=225^{\circ}$. 


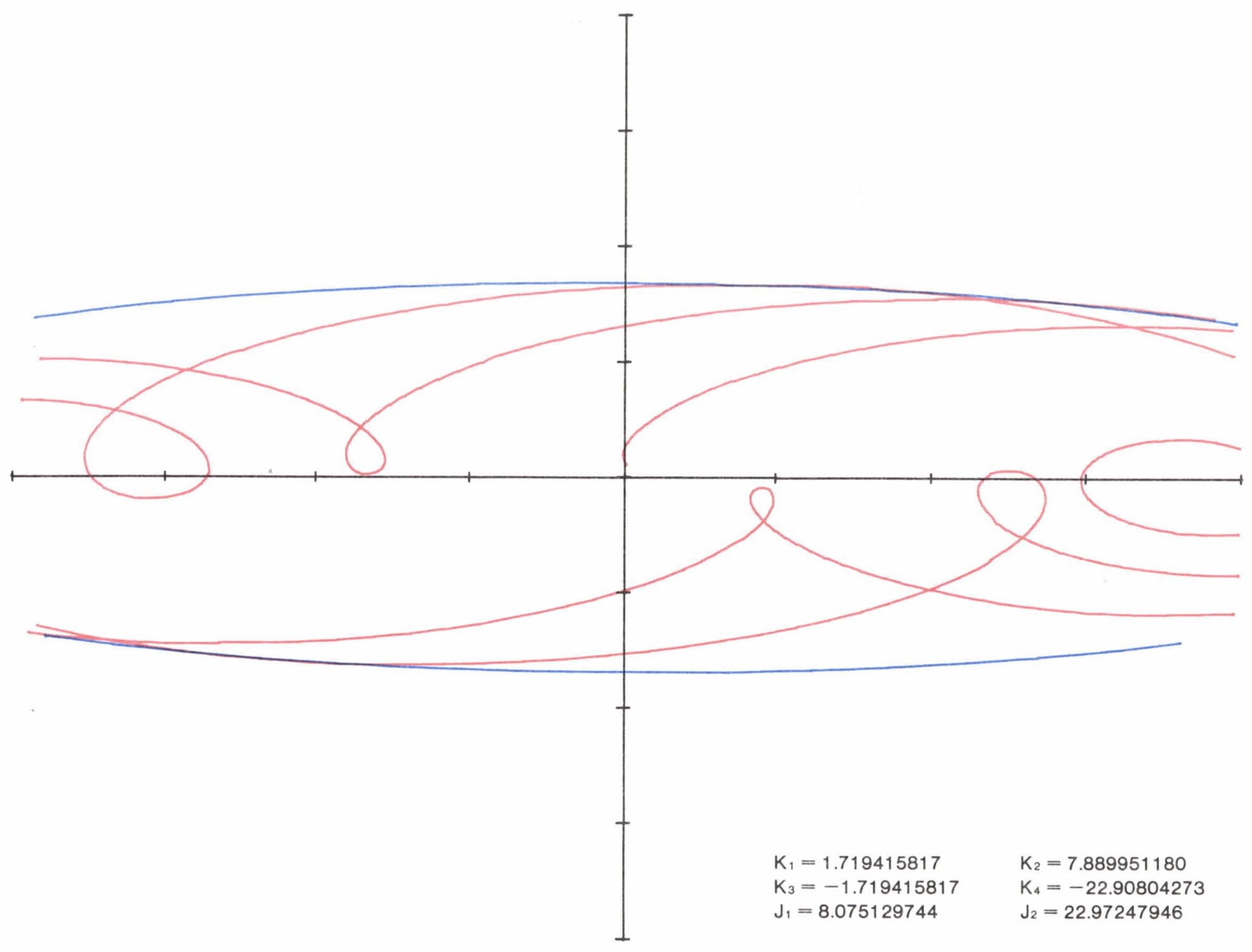

(h7) $\alpha=1, \beta=315^{\circ}, \gamma=270^{\circ}$.

FIGURE 3.-Continued. 


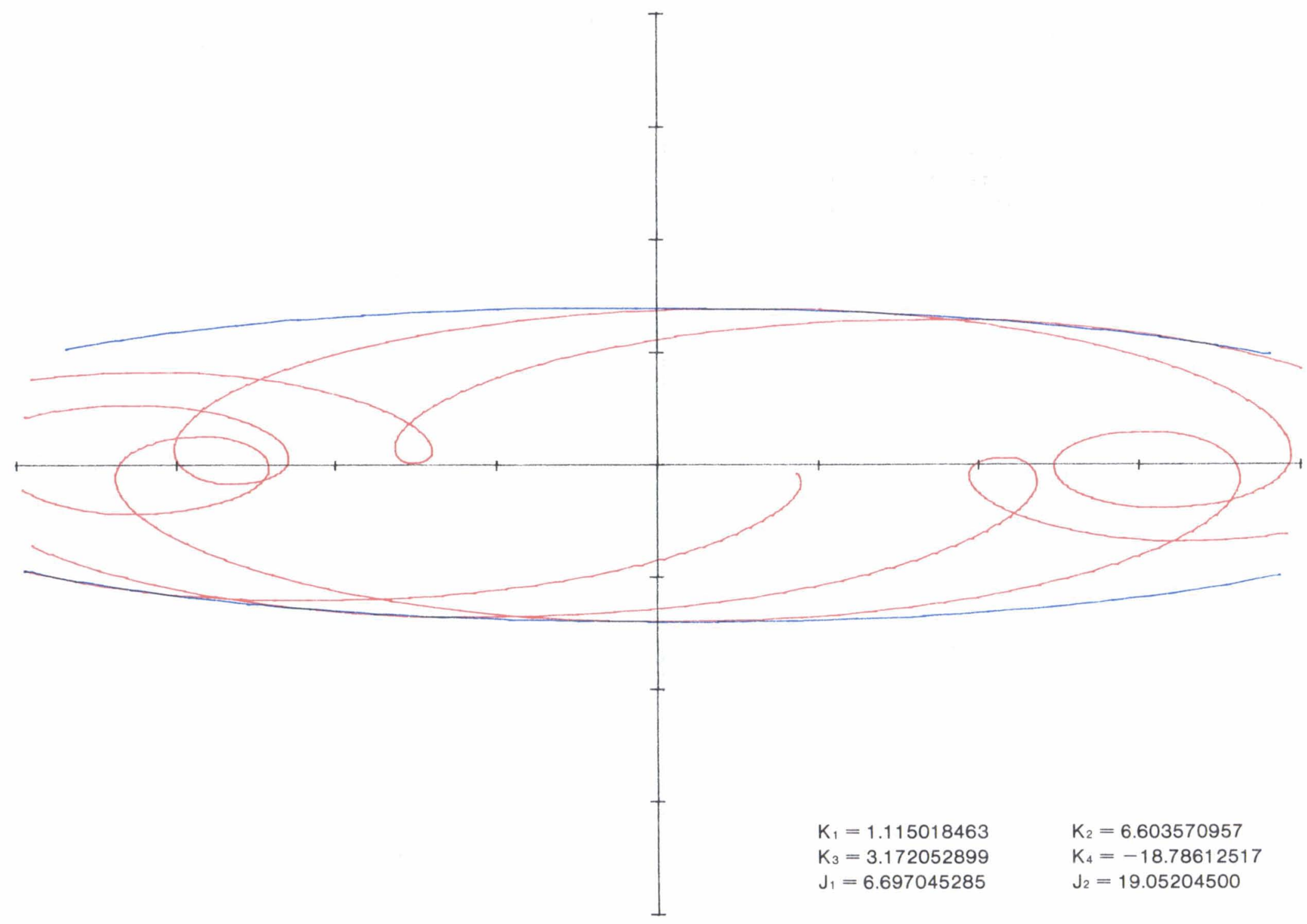

(h8) $\alpha=1, \beta=315^{\circ}, \gamma=315^{\circ}$. 


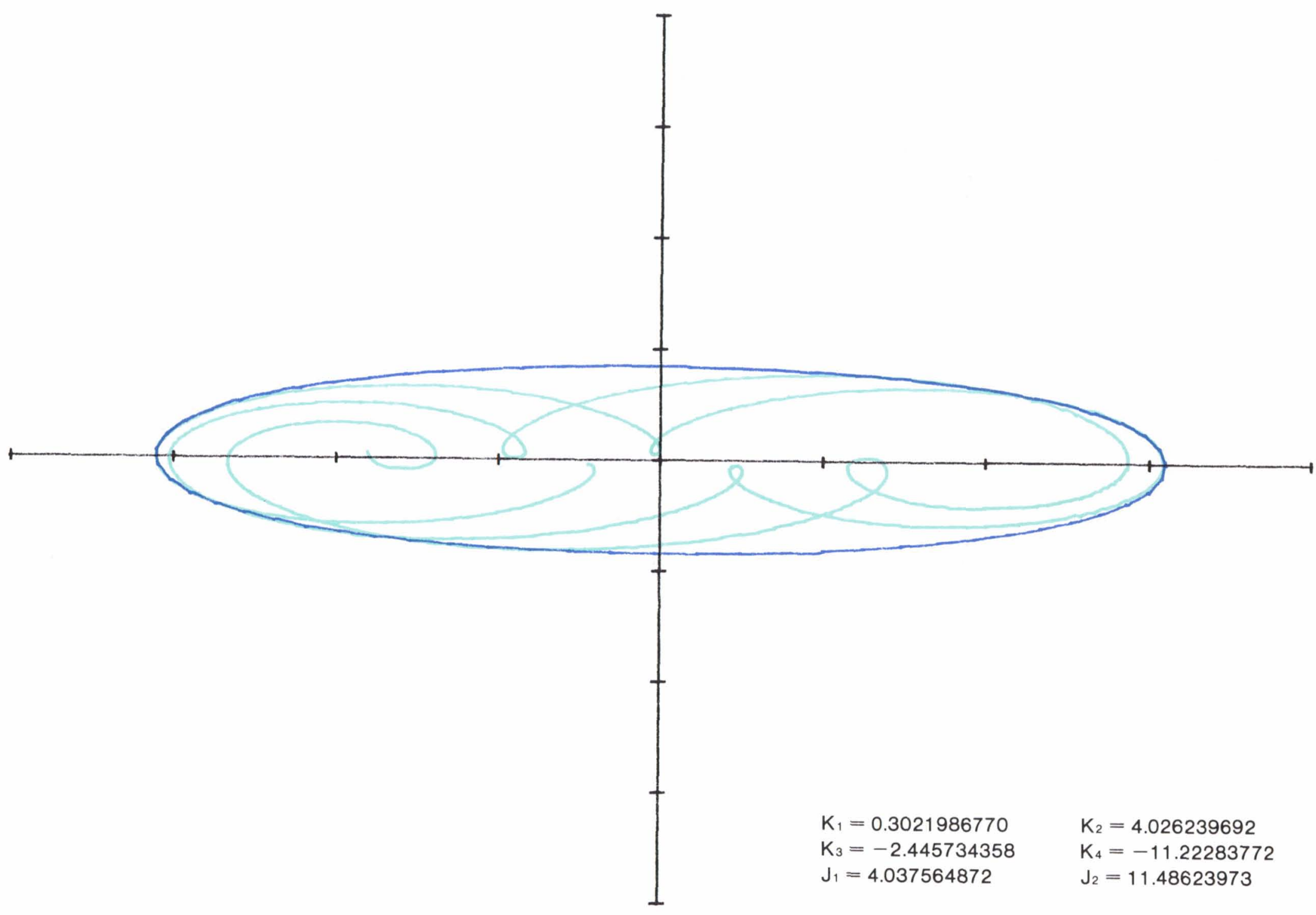

(i1) $\alpha=0.5, \beta=0^{\circ}, \gamma=225^{\circ}$.

FIGURE 3.-Continued. 


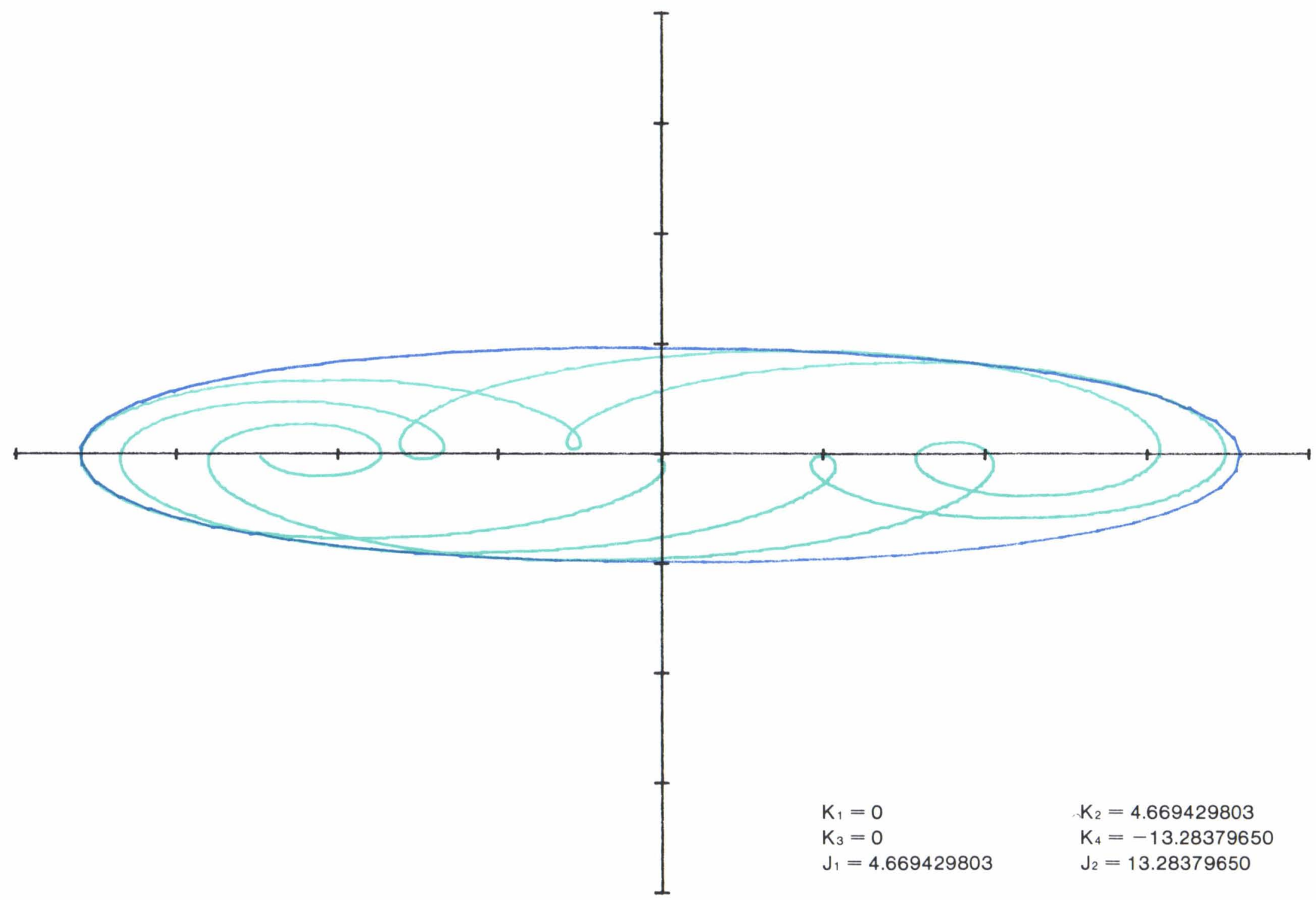

(i2) $\alpha=0.5, \beta=0^{\circ}, \gamma=270^{\circ}$. 


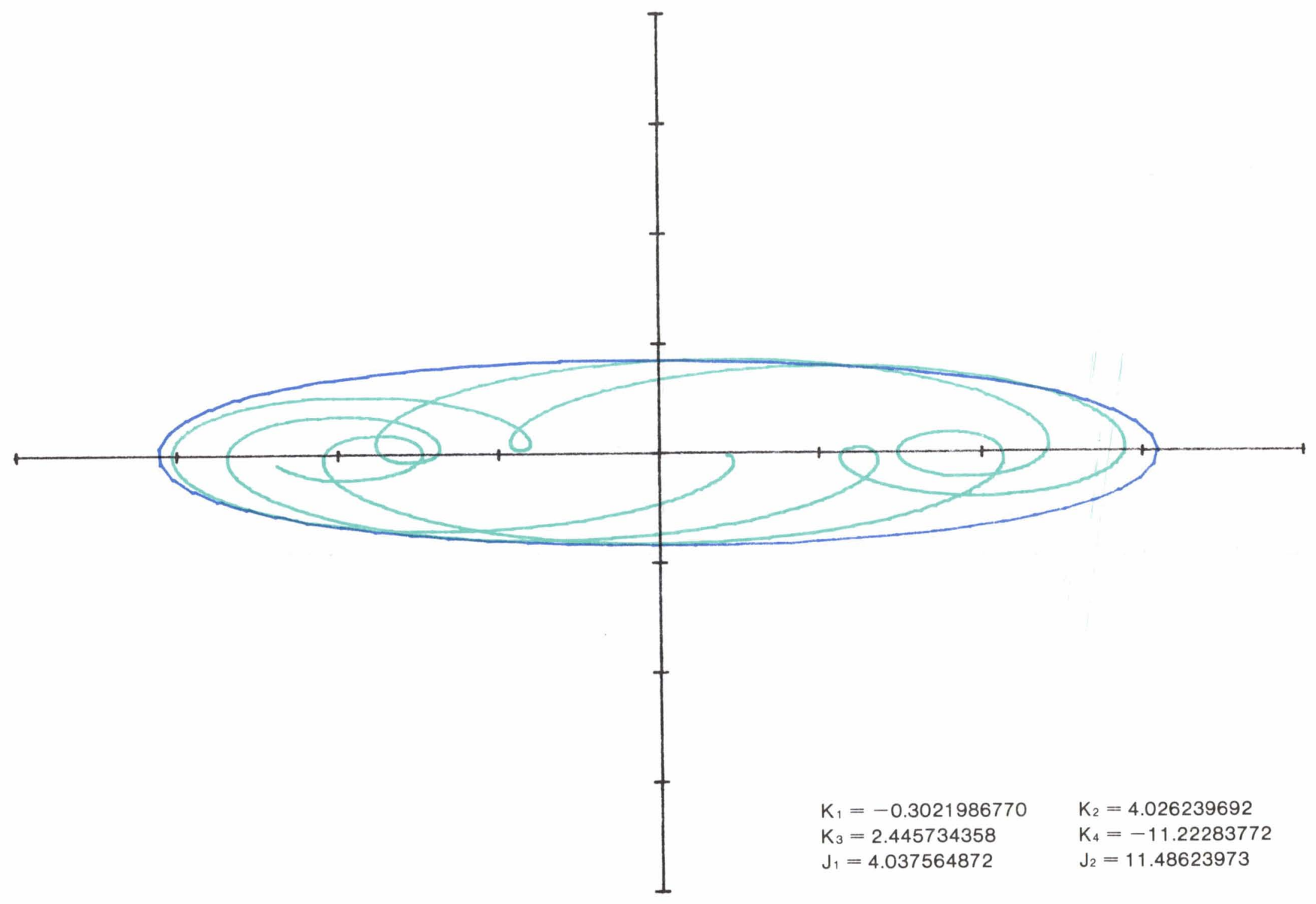

(i3) $\alpha=0.5, \beta=0^{\circ}, \gamma=315^{\circ}$.

FIGURE 3.-Continued. 


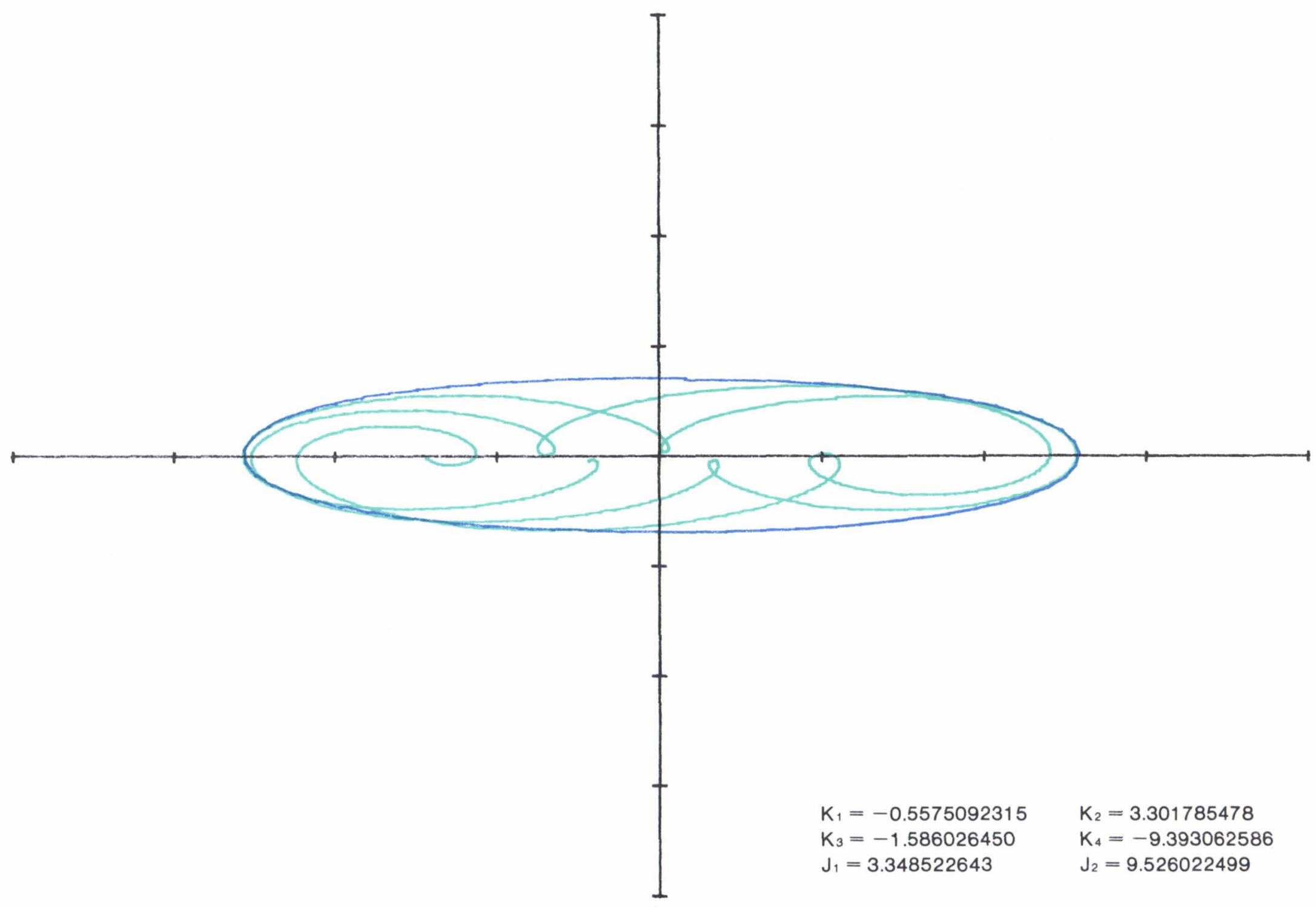

(j1) $\alpha=0.5, \beta=45^{\circ}, \gamma=225^{\circ}$. 


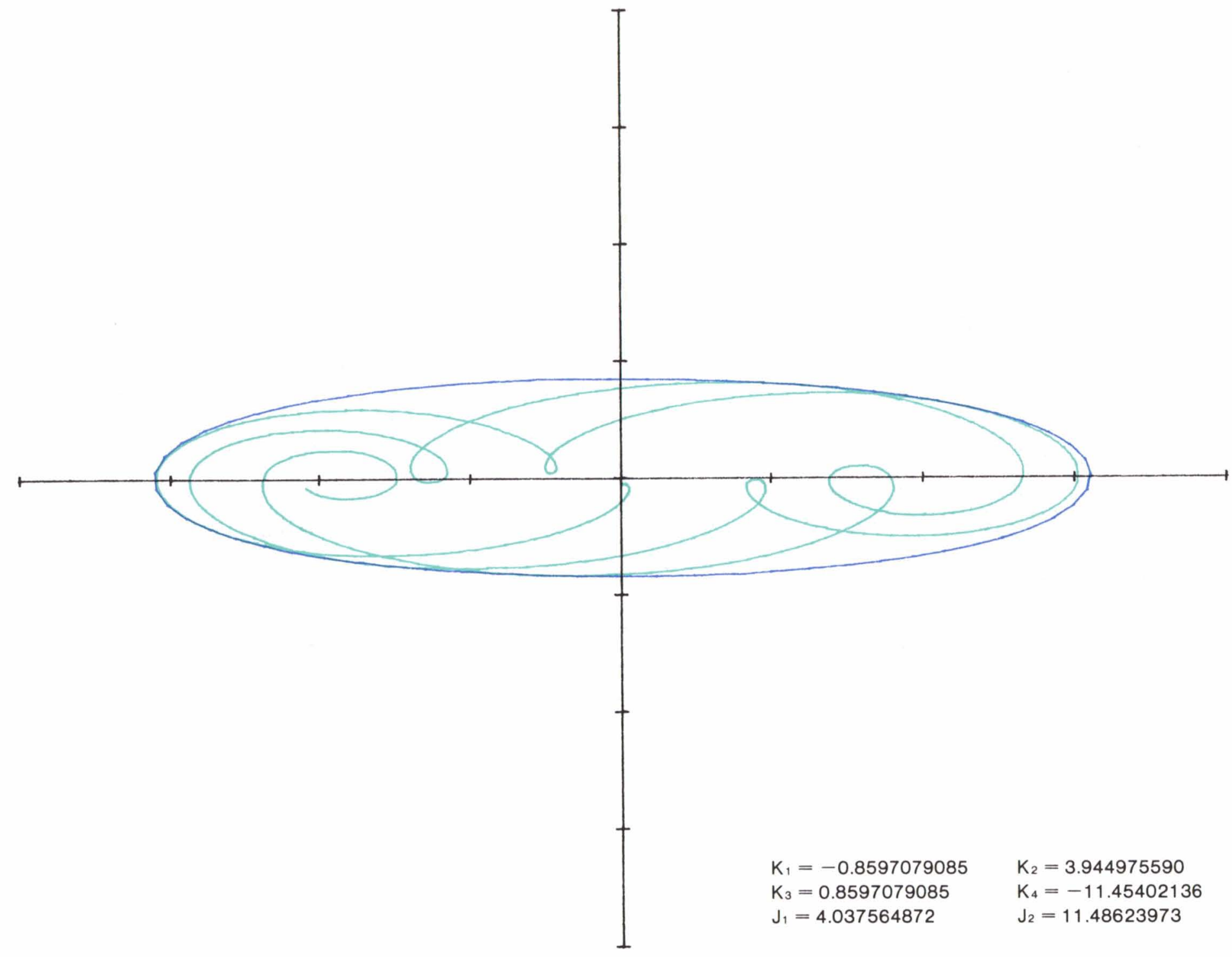

(j2) $\alpha=0.5, \beta=45^{\circ}, \gamma=270^{\circ}$.

FIGURE 3.-Continued. 


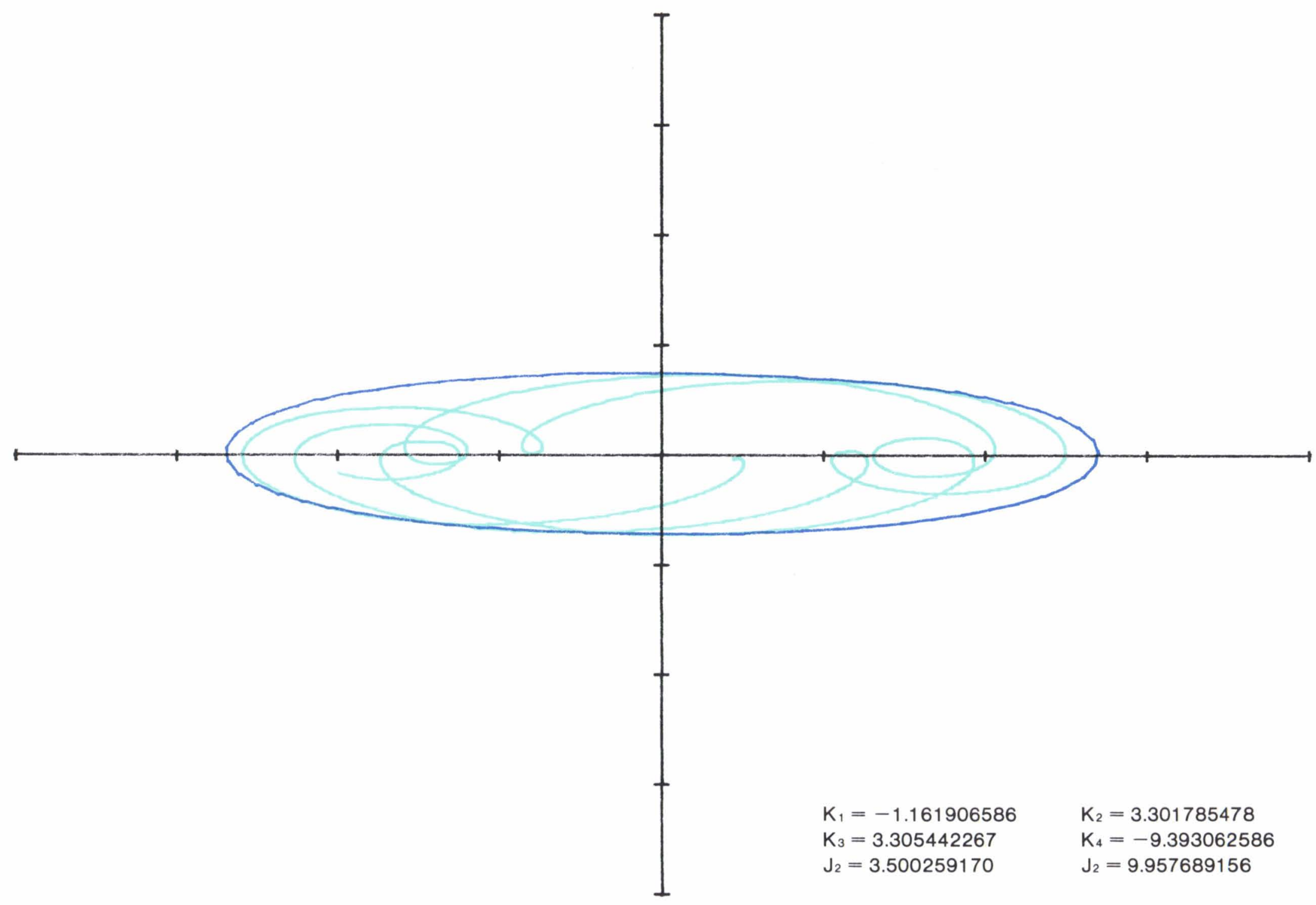

(j3) $\alpha=0.5, \beta=45^{\circ}, \gamma=315^{\circ}$. 


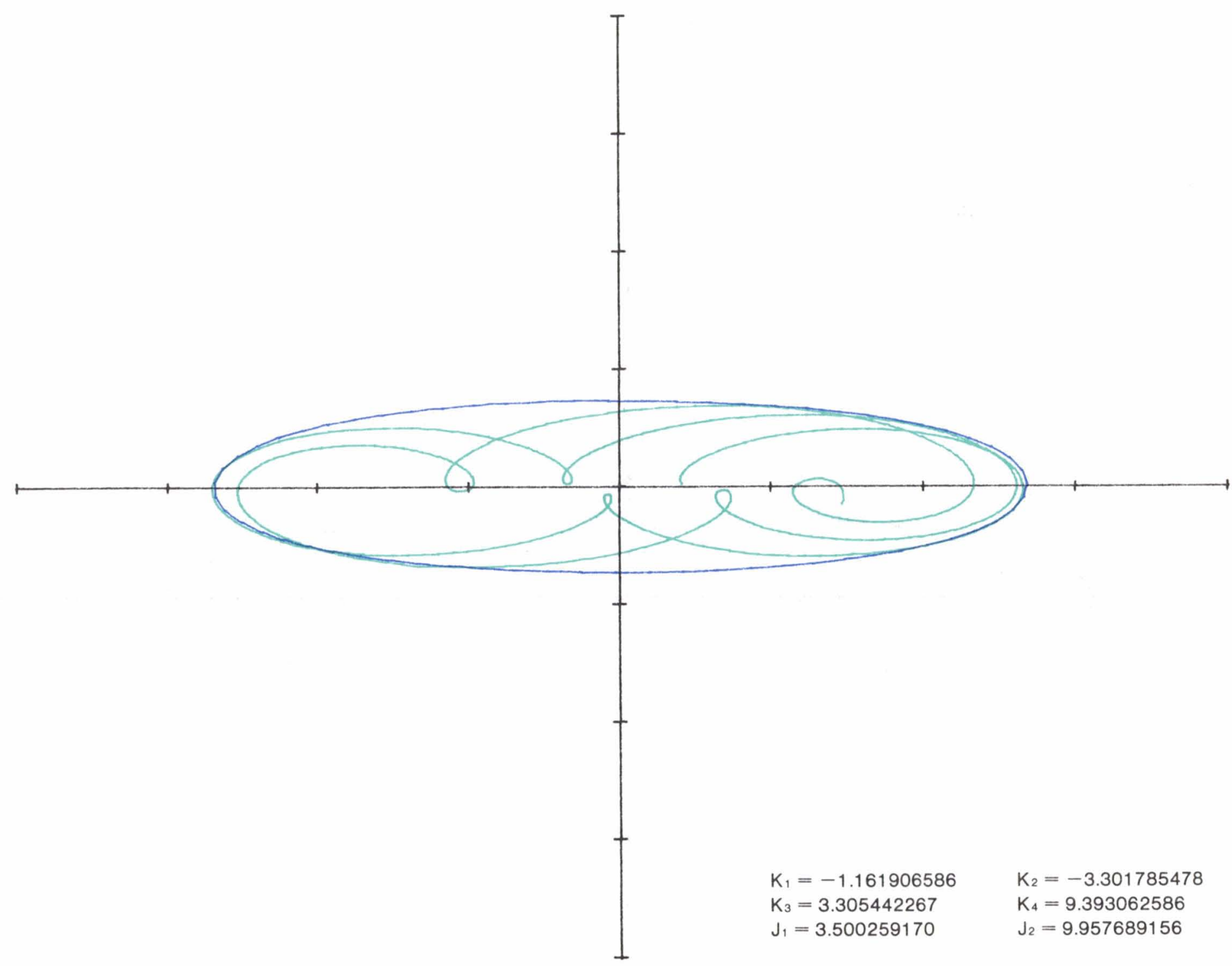

(k1) $\alpha=0.5, \beta=135^{\circ}, \gamma=45^{\circ}$.

FIGURE 3.-Continued. 


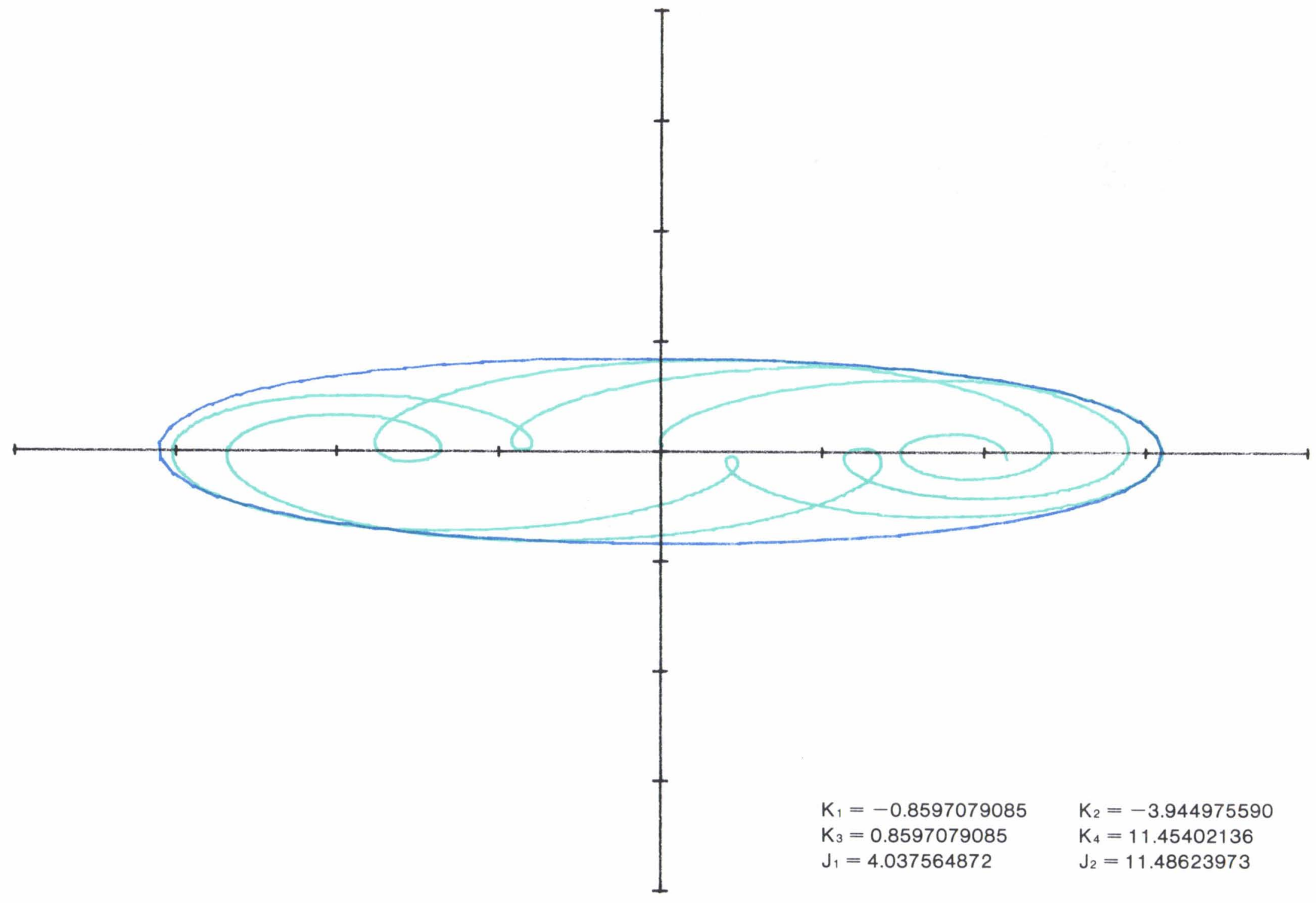

(k2) $\alpha=0.5, \beta=135^{\circ}, \gamma=90^{\circ}$. 


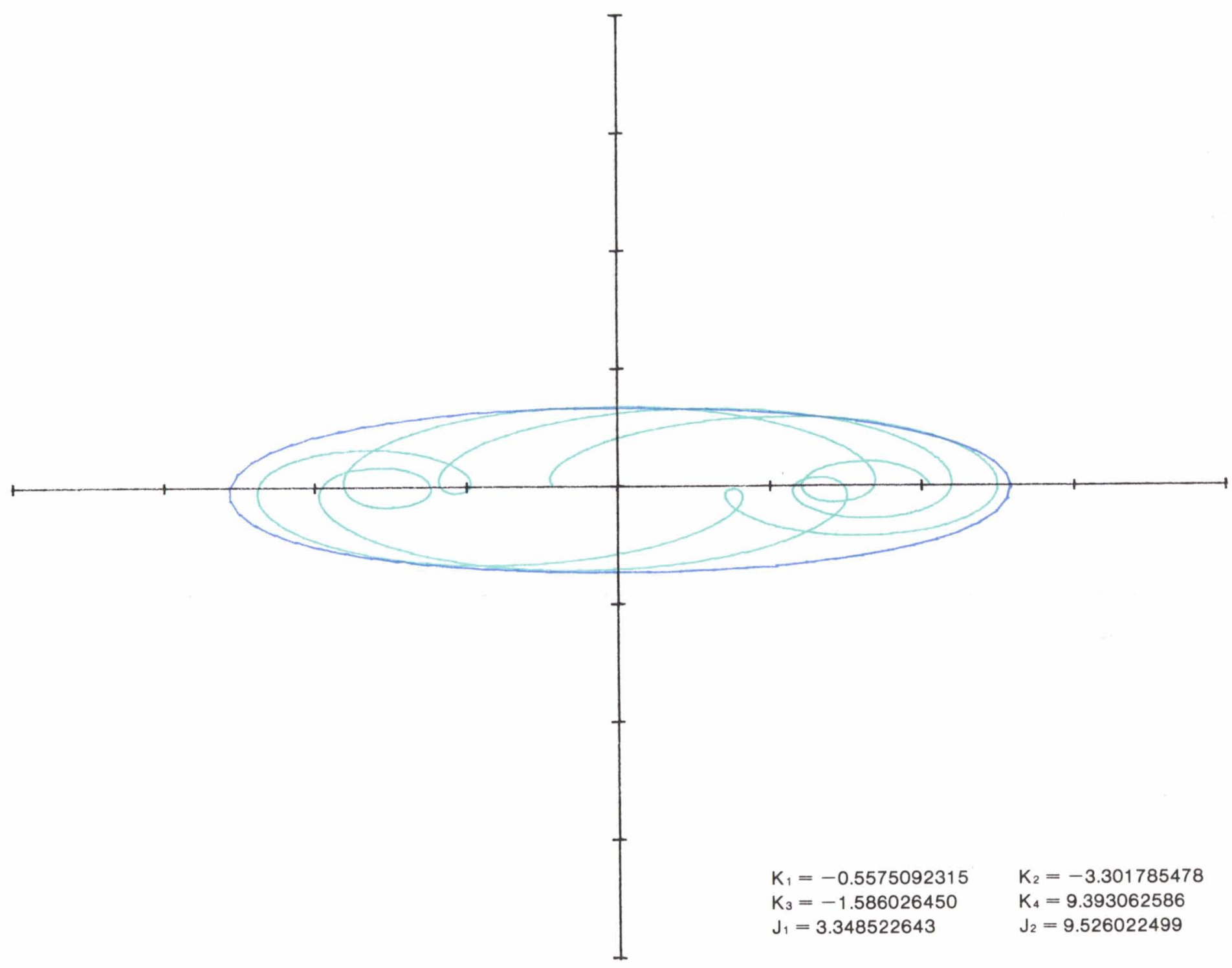

(k3) $\alpha=0.5, \beta=135^{\circ}, \gamma=135^{\circ}$.

FIGURE 3.-Continued. 


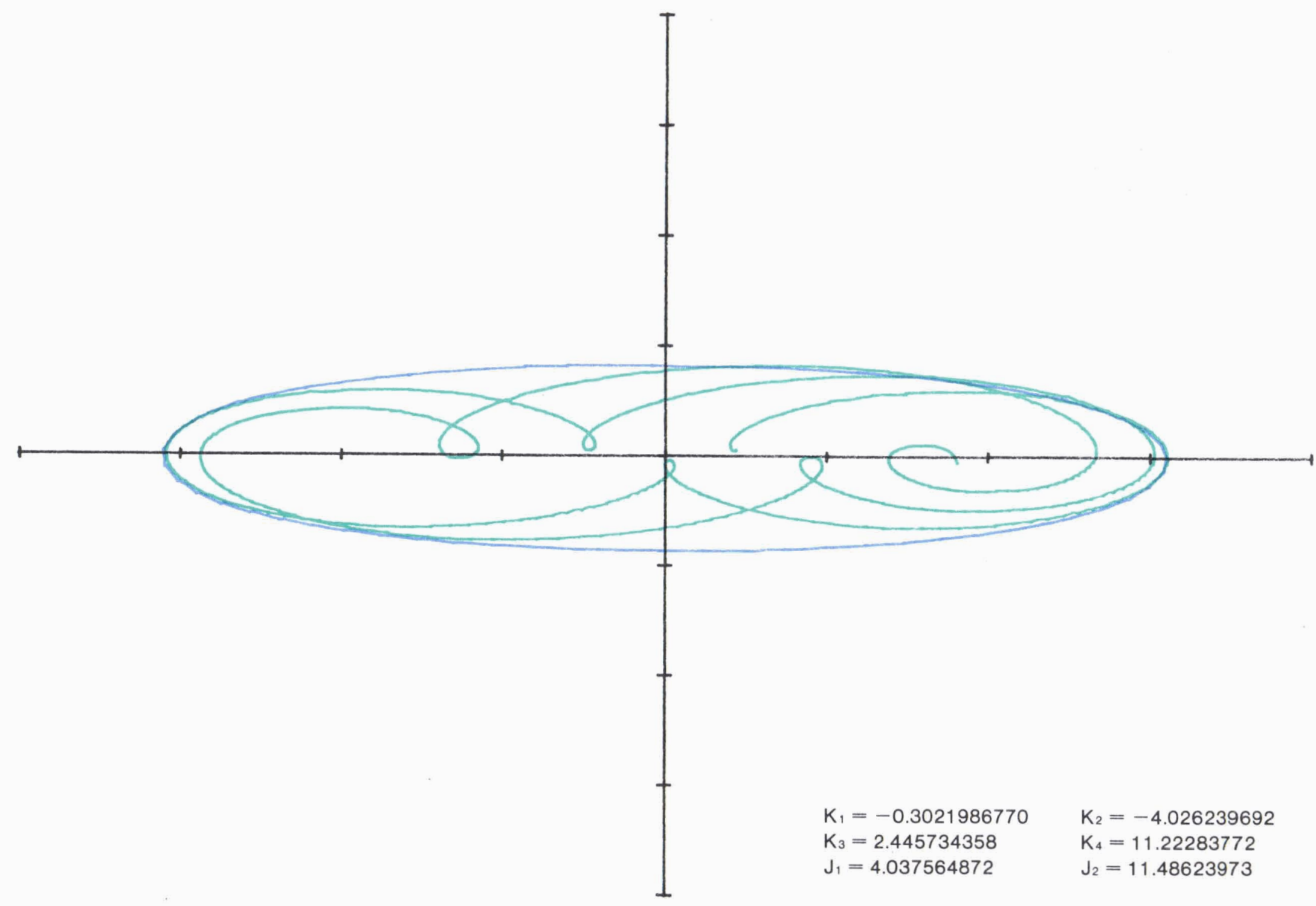

(11) $\alpha=0.5, \beta=180^{\circ}, \gamma=45^{\circ}$.

FIGURE 3.-Continued. 


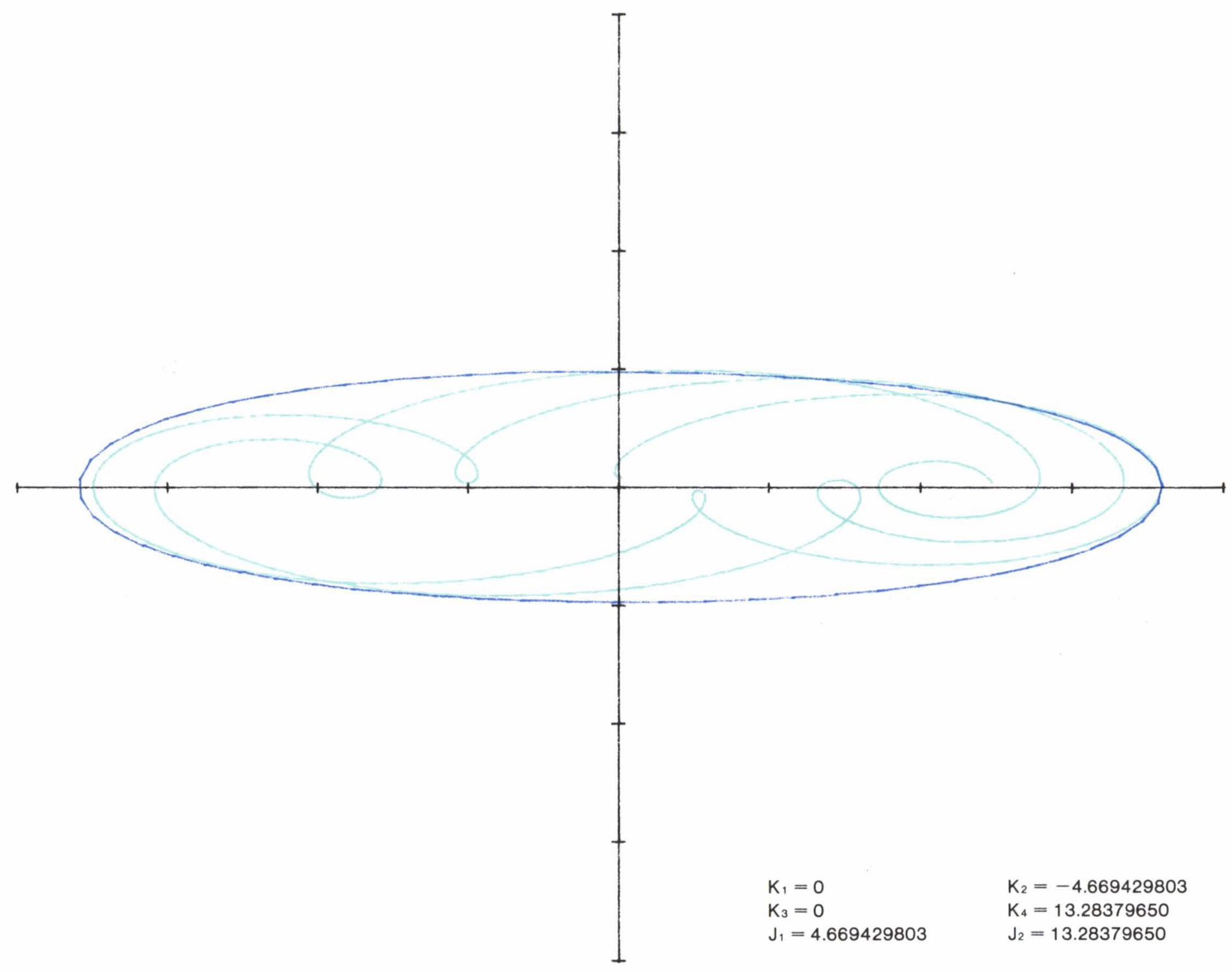

(12) $\alpha=0.5, \beta=180^{\circ}, \gamma=90^{\circ}$.

FIGURE 3.-Continued. 


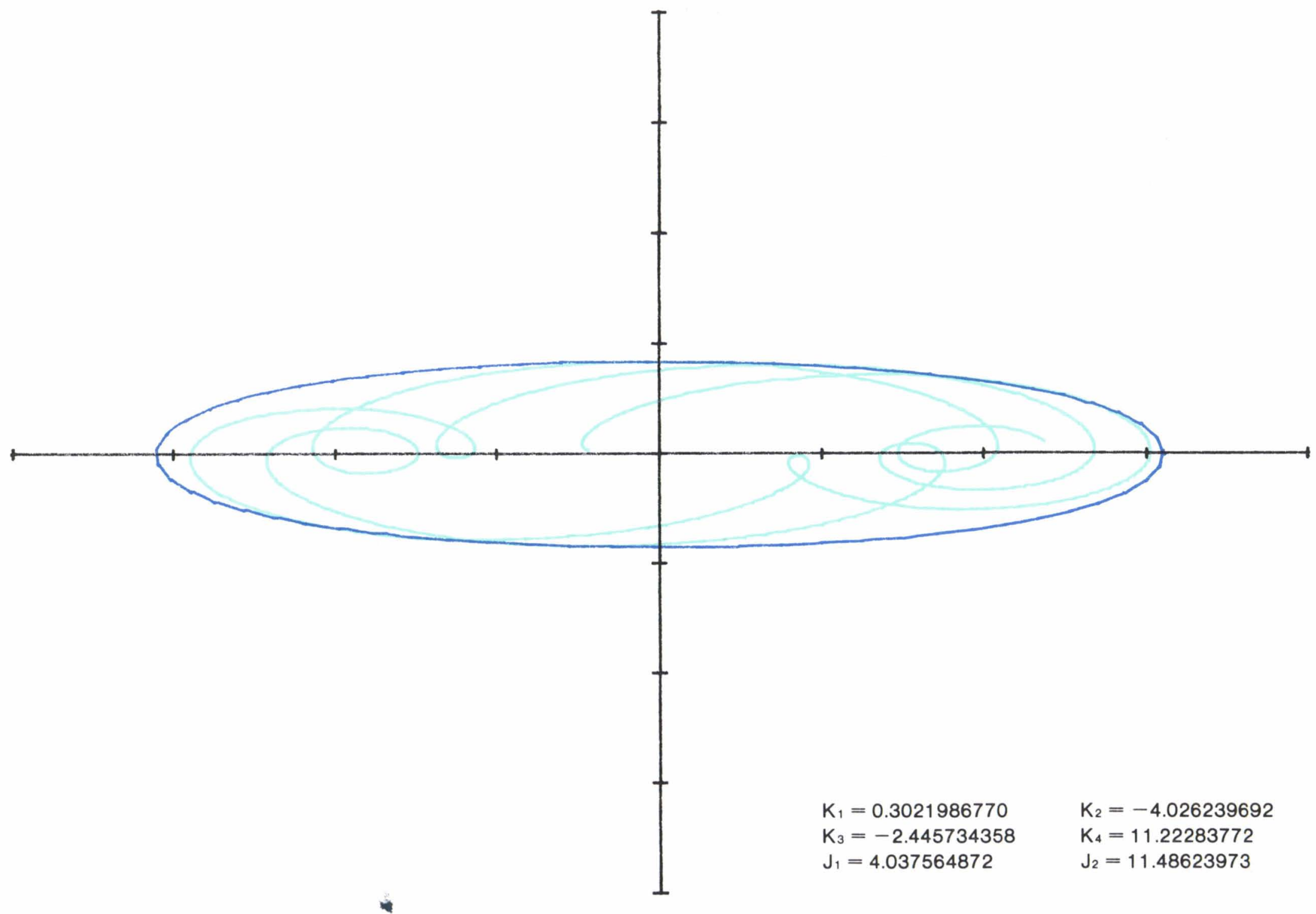

(13) $\alpha=0.5, \beta=180^{\circ}, \gamma=135^{\circ}$.

FIGURE 3.-Continued. 


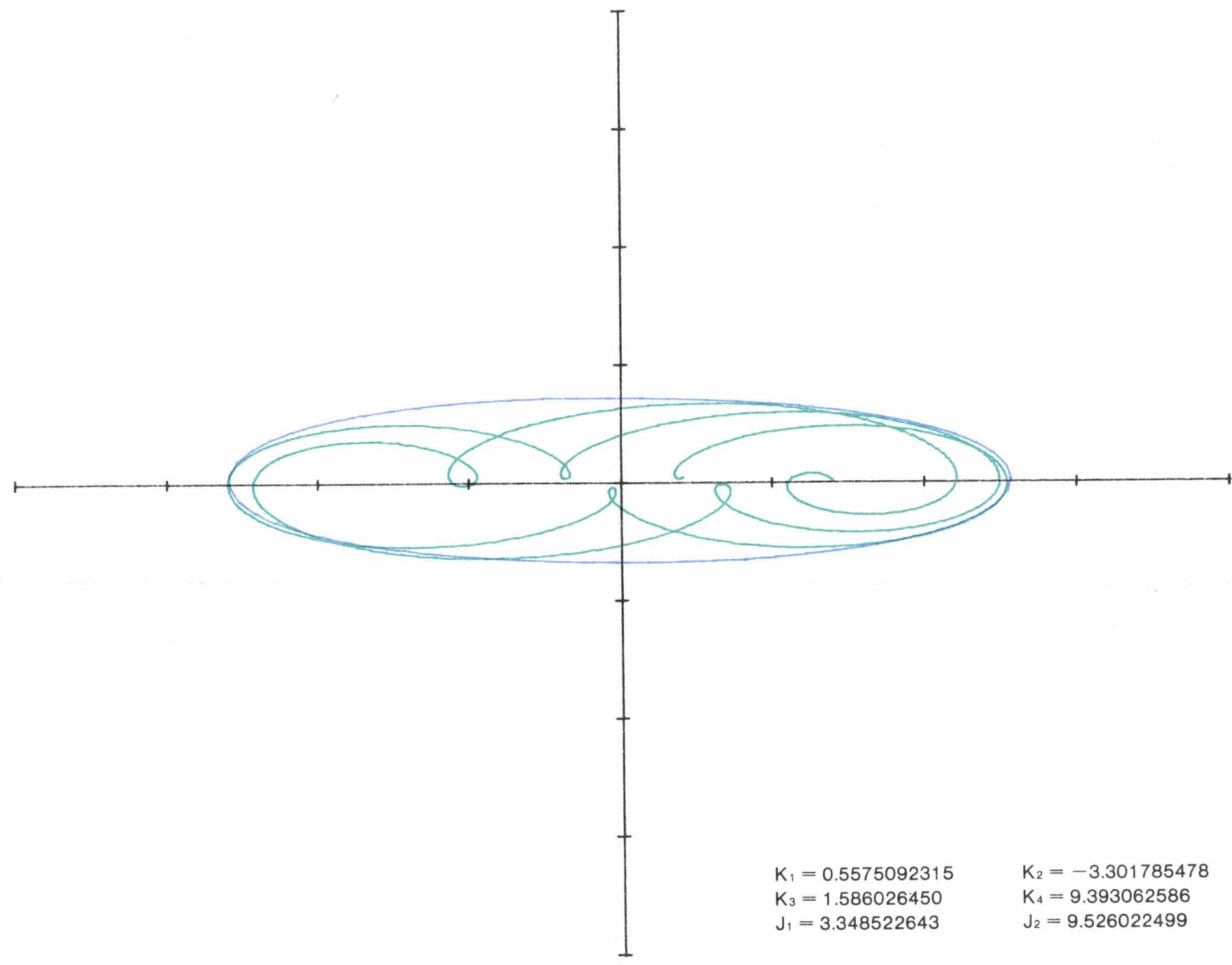

(m1) $\alpha=0.5, \beta=225^{\circ}, \gamma=45^{\circ}$.

FIGURE 3.-Continued. 


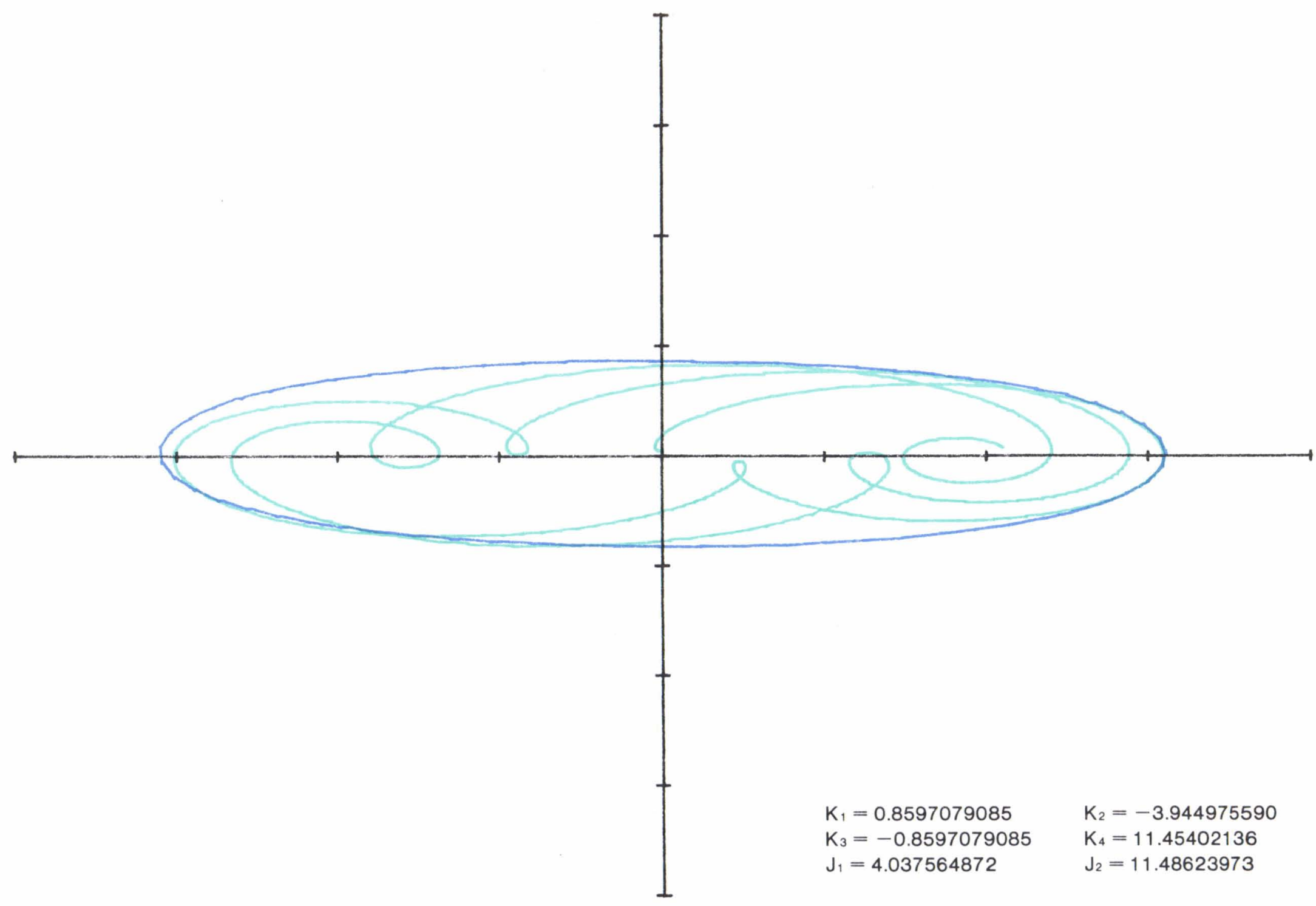

(m2) $\alpha=0.5, \beta=225^{\circ}, \gamma=90^{\circ}$.

FIGURE 3.-Continued. 


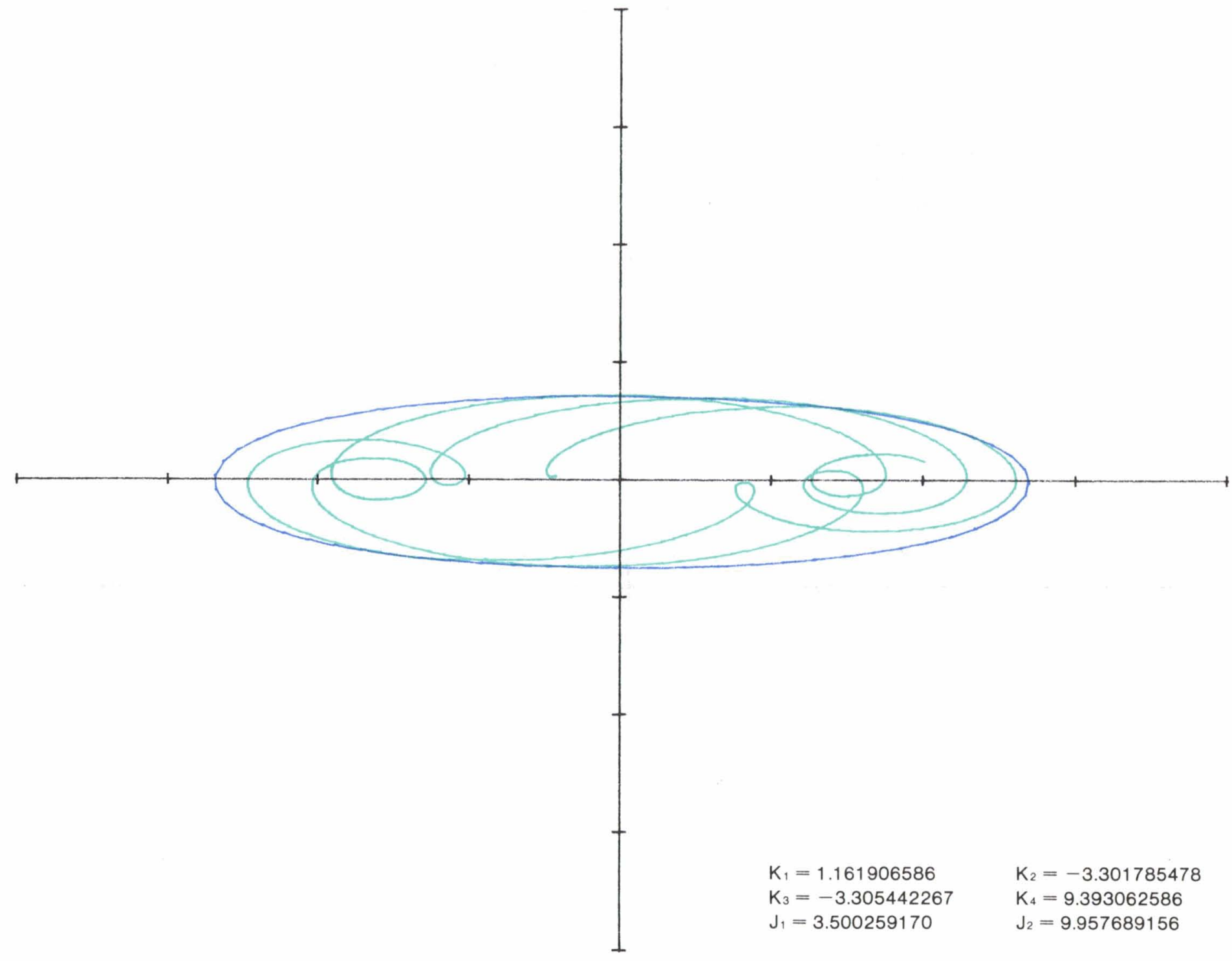

(m3) $\alpha=0.5, \beta=225^{\circ}, \gamma=135^{\circ}$.

FIGURE 3.-Continued. 


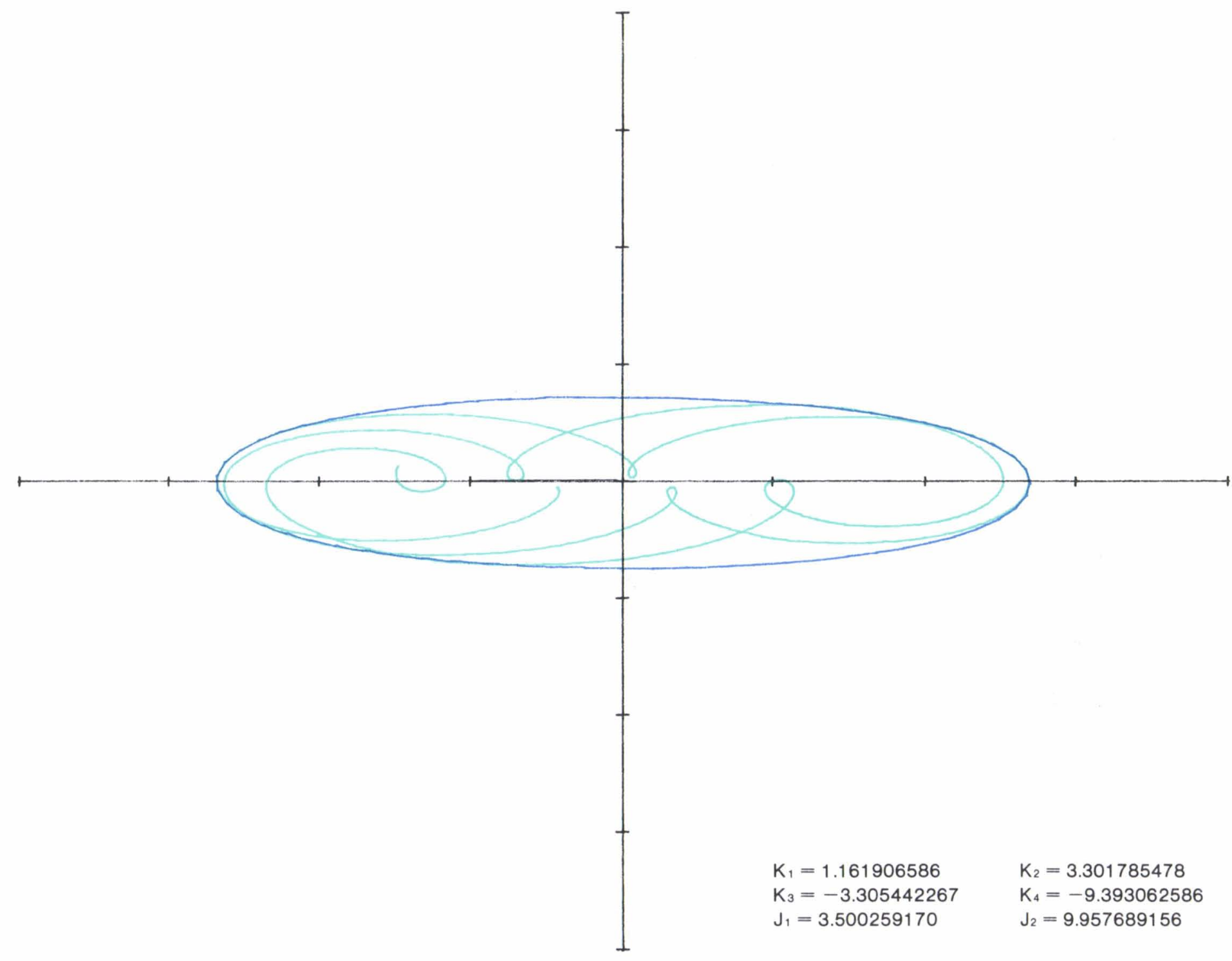

(n1) $\alpha=0.5, \beta=315^{\circ}, \gamma=225^{\circ}$. 


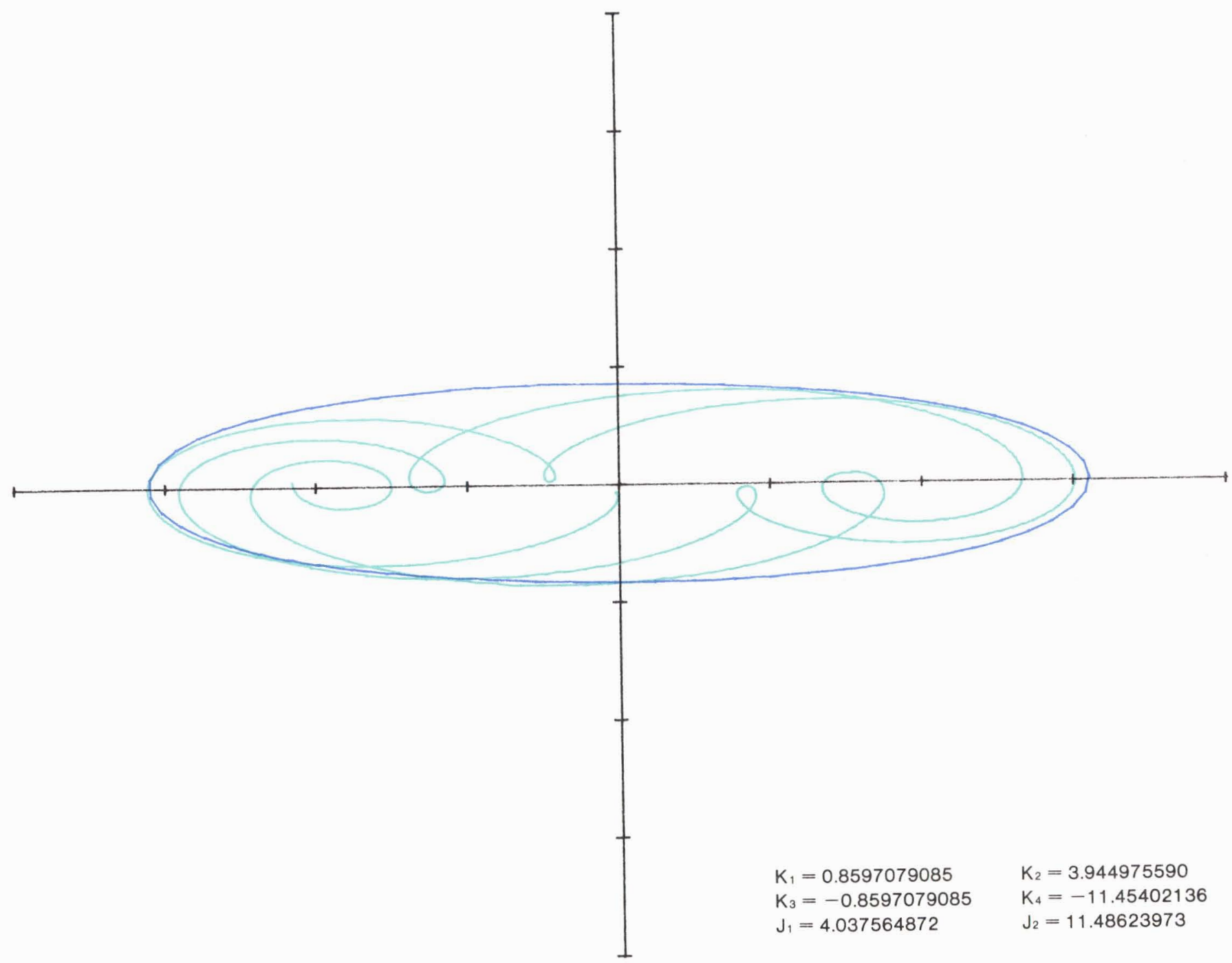

(n2) $\alpha=0.5, \beta=315^{\circ}, \gamma=270^{\circ}$.

FIGURE 3.-Continued. 


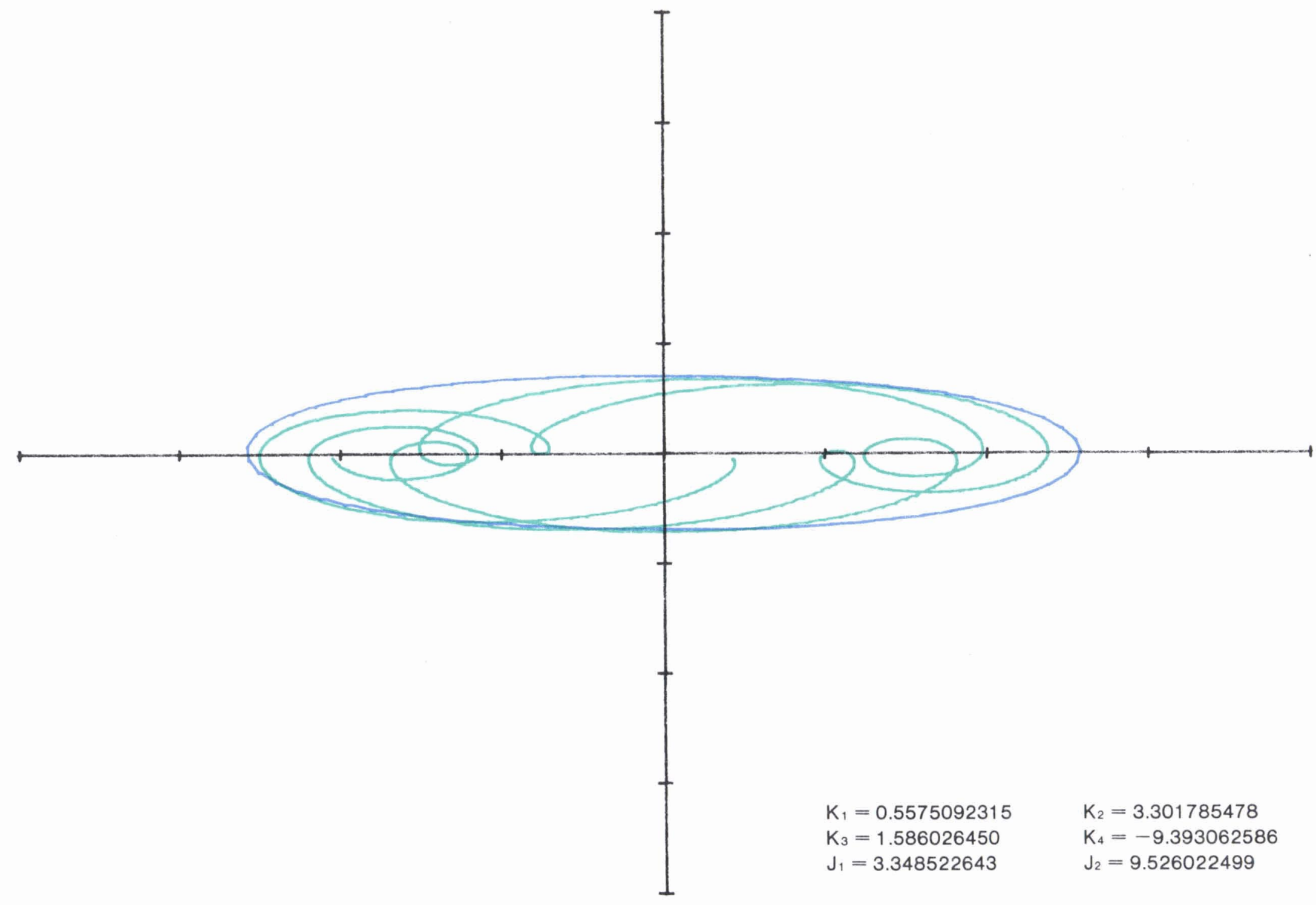

(n3) $\alpha=0.5, \beta=315^{\circ}, \gamma=315^{\circ}$ 


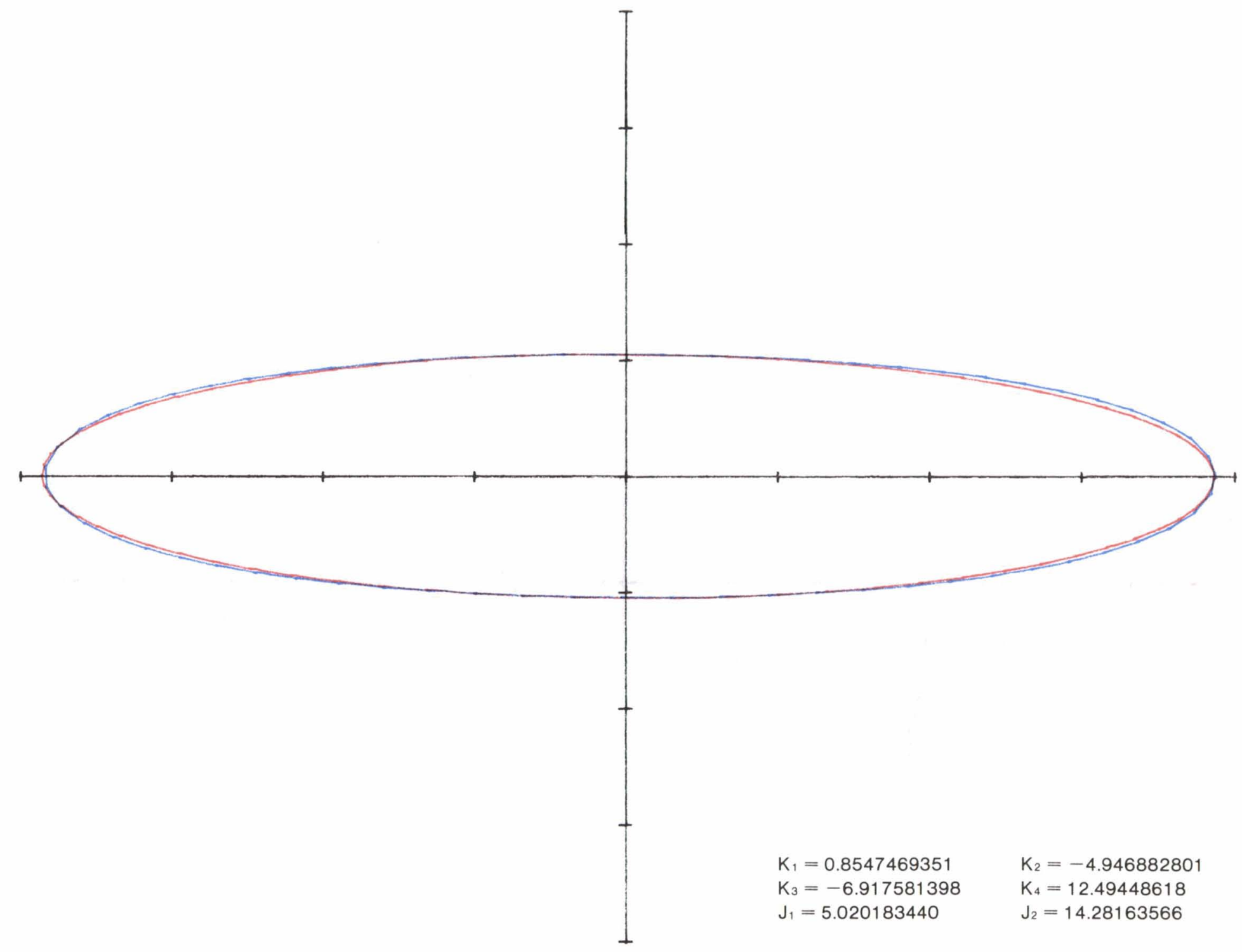

(a) $\alpha=1, \beta=180^{\circ}, \gamma=180^{\circ}$.

FIGURE 4.-Comparison of approximate and exact bounds. 


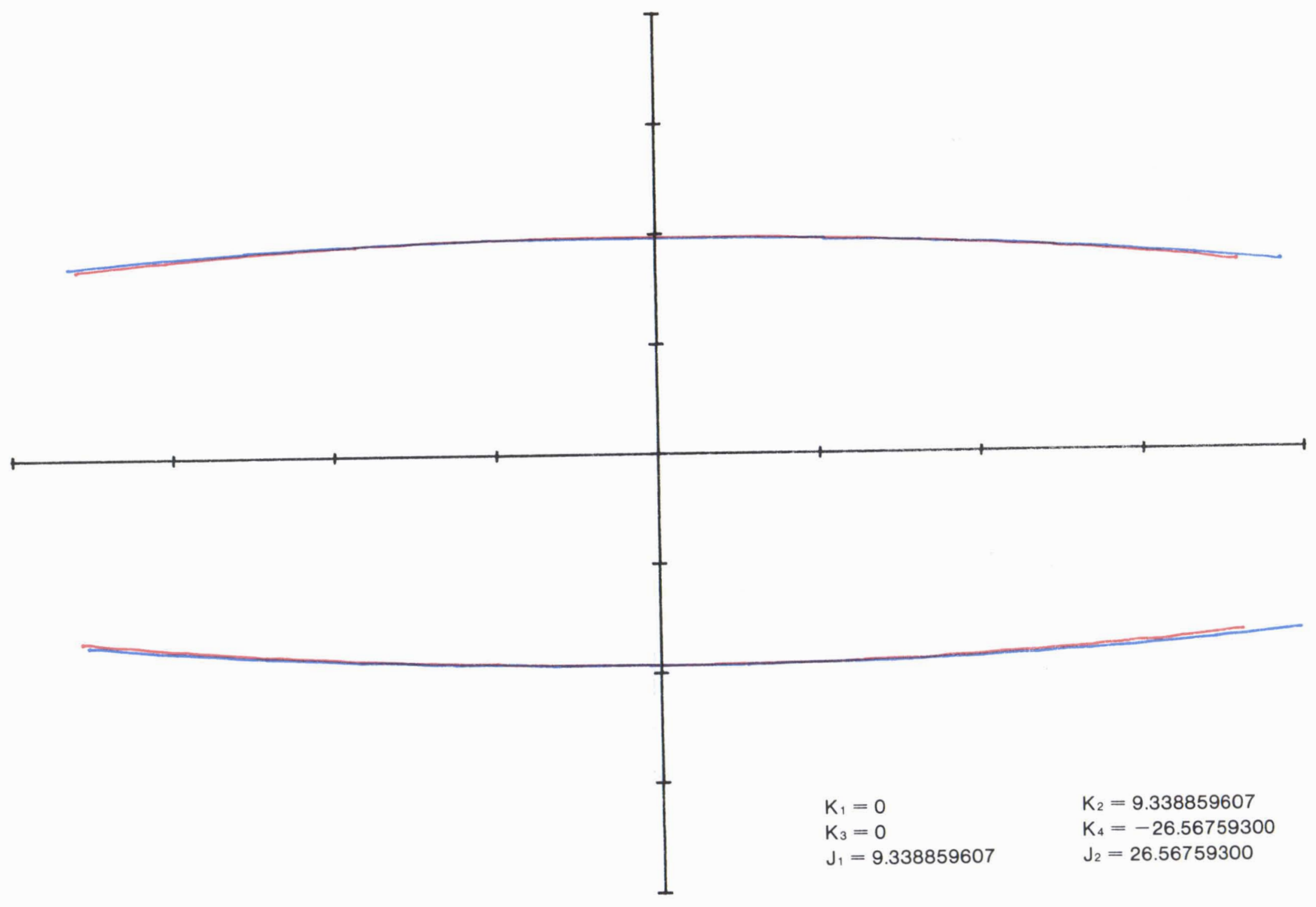

(b) $\alpha=1, \beta=0^{\circ}, \gamma=270^{\circ}$.

FIGURE 4.-Continued. 


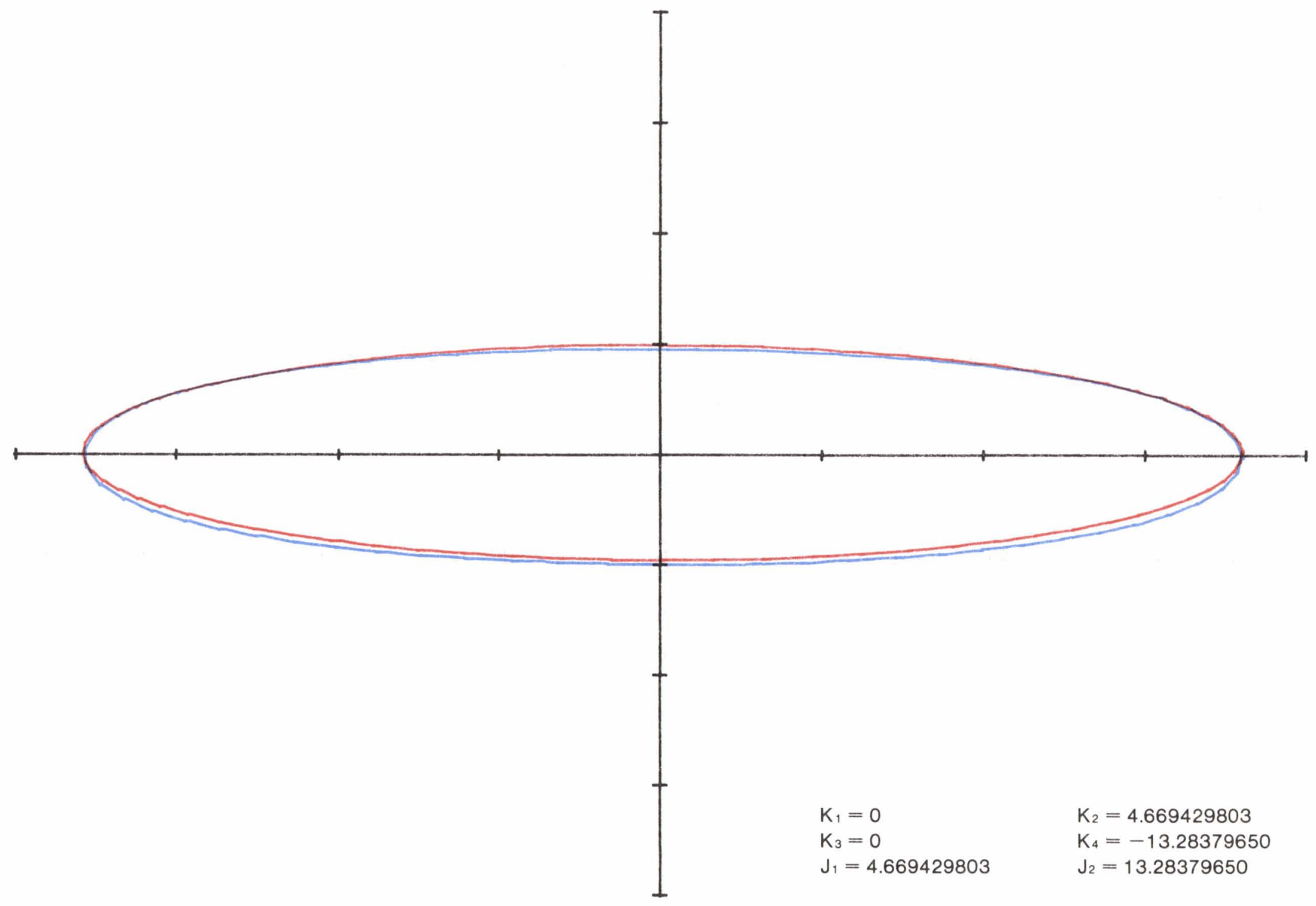

(c) $\alpha=0.5, \beta=0^{\circ}, \gamma=270^{\circ}$.

FIGURE 4.-Continued. 


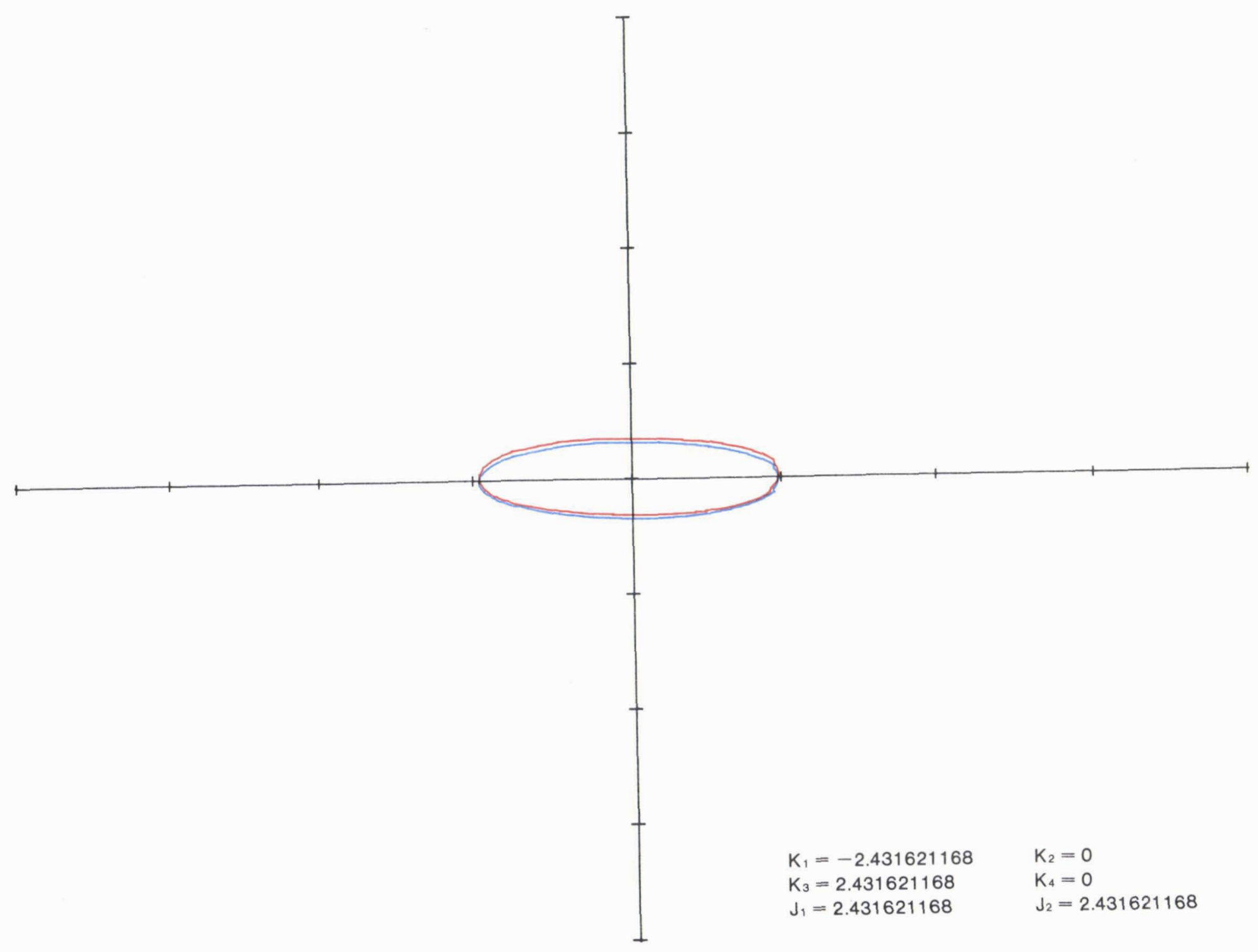

(d) $\alpha=0, \beta=0, \gamma=90^{\circ}$. 


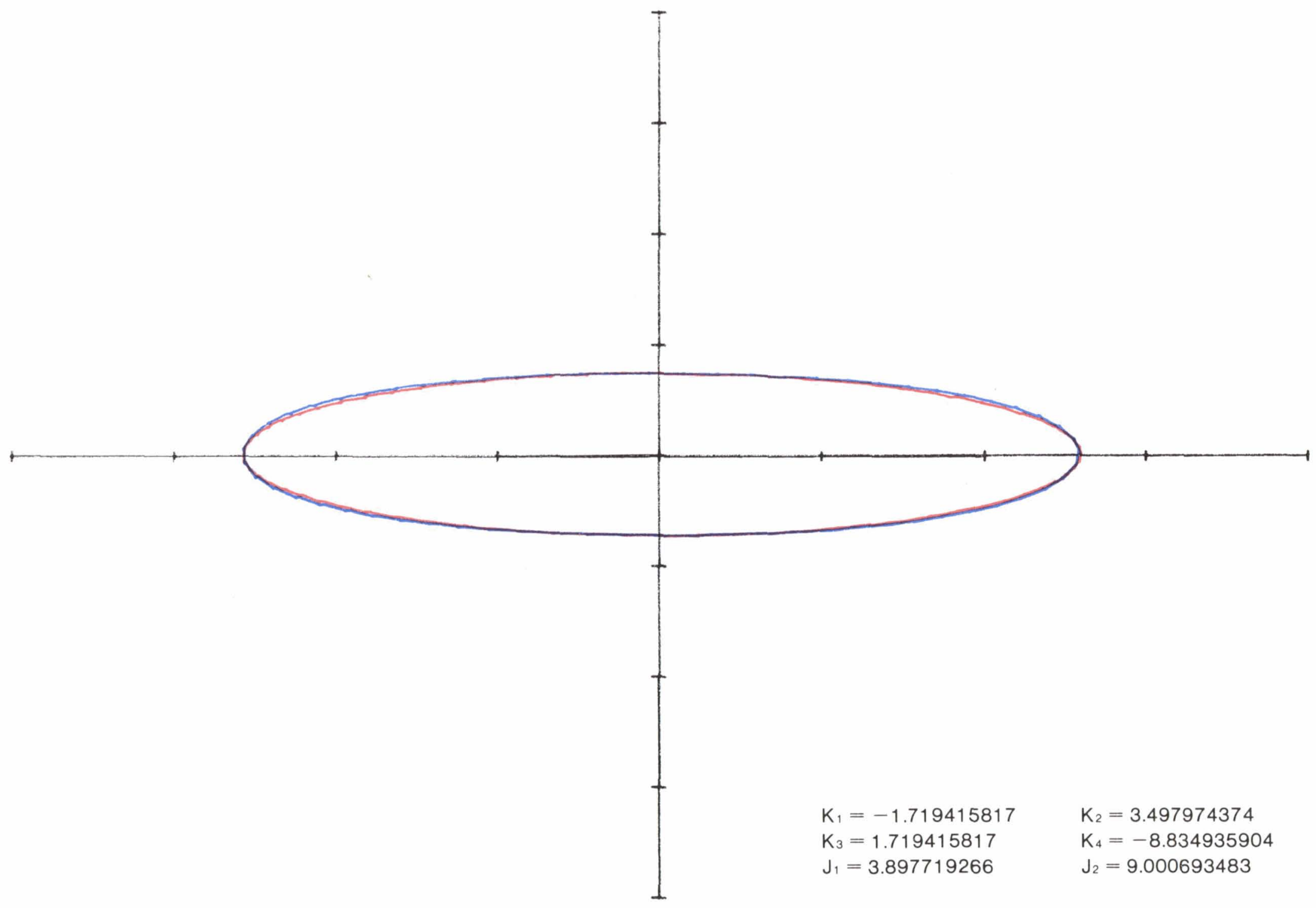

(e) $\alpha=0, \beta=0, \gamma=45^{\circ}$.

FIGURE 4.-Continued. 


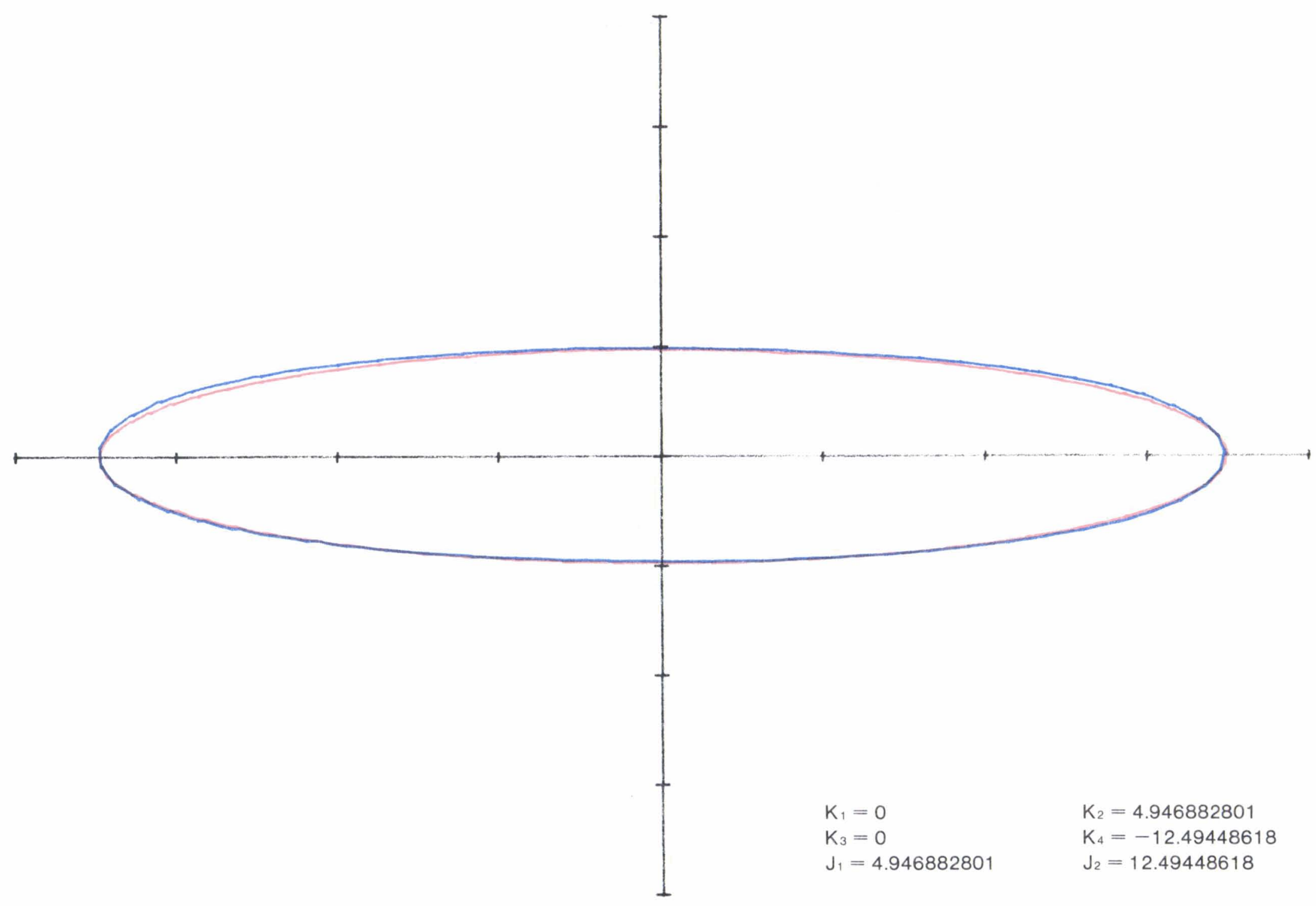




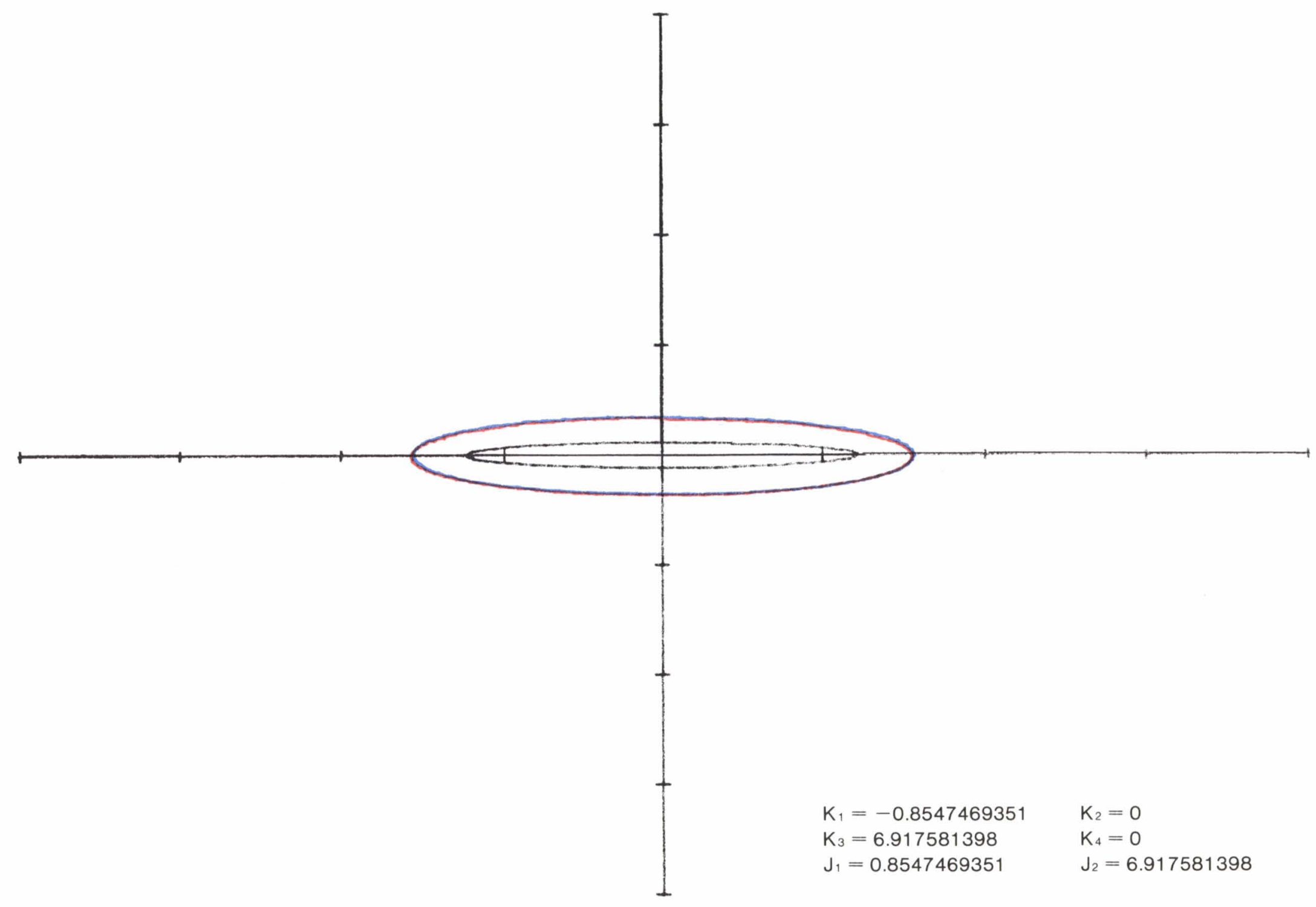

(g) $\alpha=0, \beta=0, \gamma=0^{\circ}$.

FIGURE 4.-Continued. 


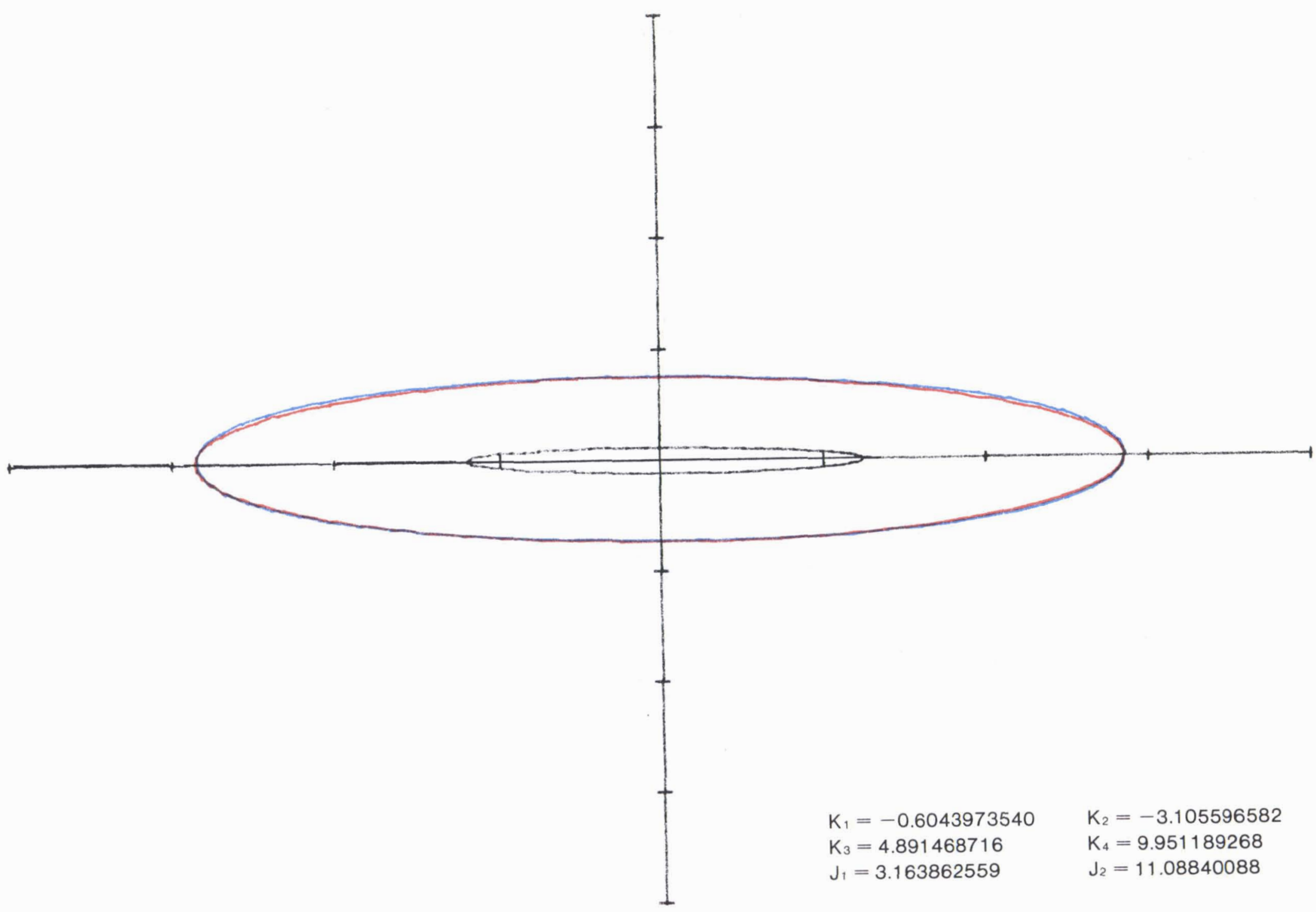

(h) $\alpha=\mathbf{0}, \beta=0, \gamma=45^{\circ}$. 


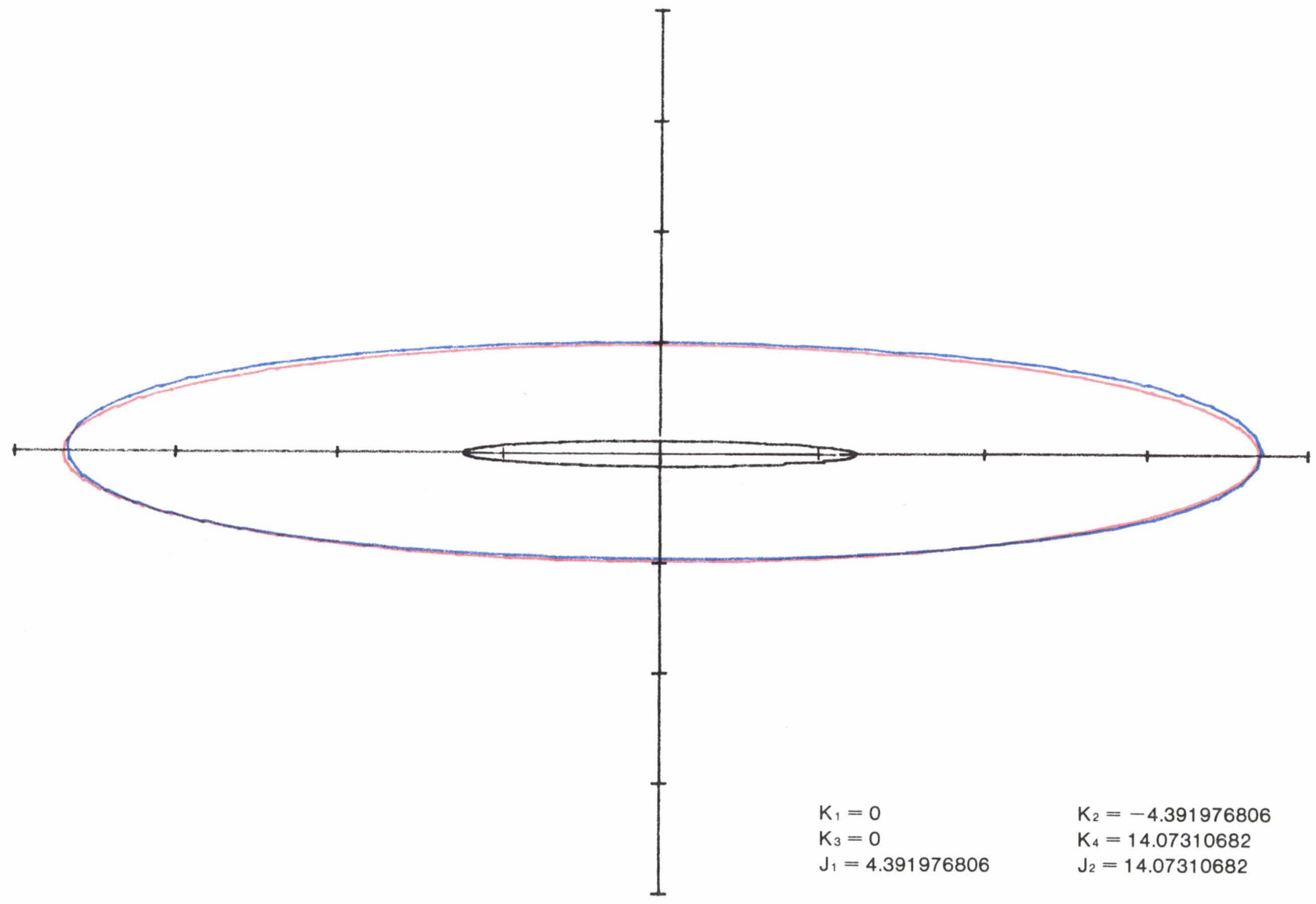

(i) $\alpha=0, \beta=0, \gamma=90^{\circ}$.

FIGURE 4.-Continued. 


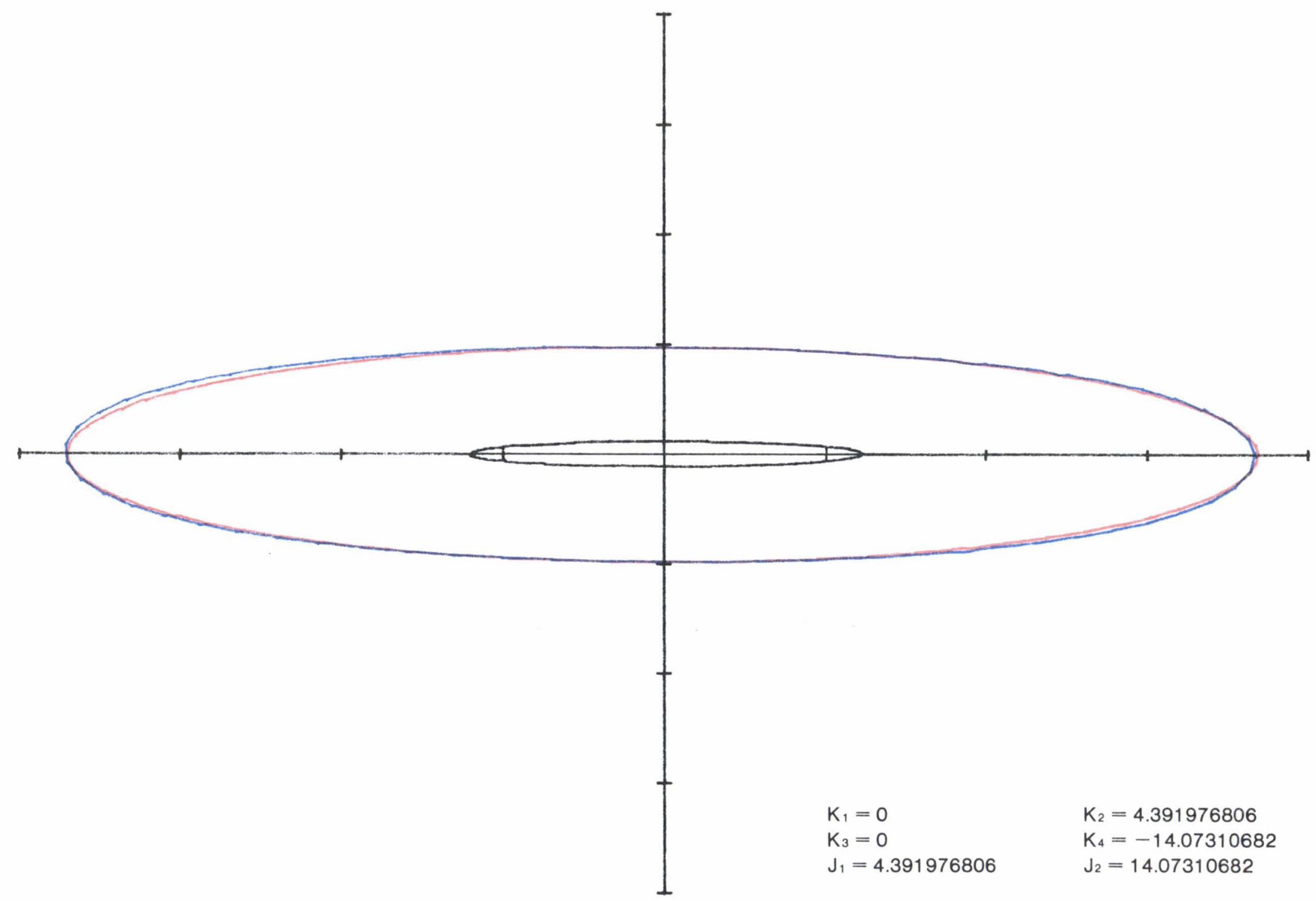

(j) $\alpha=0, \beta=0, \gamma=270^{\circ}$. 


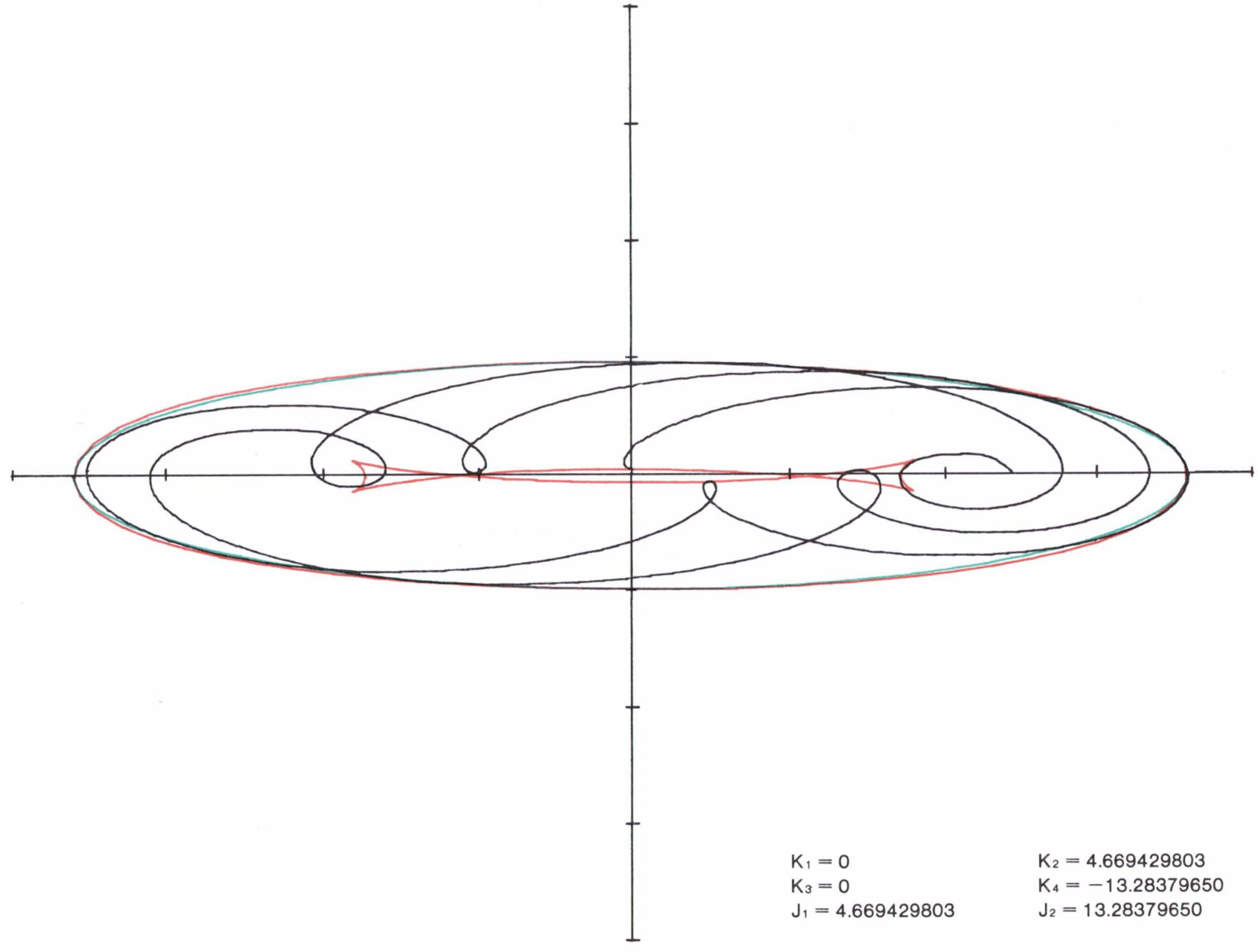

FIGURE 5.-Typical inner bounding curve showing excluded region $\left(\alpha=0.5, \beta=0, \gamma=270^{\circ}\right)$. 


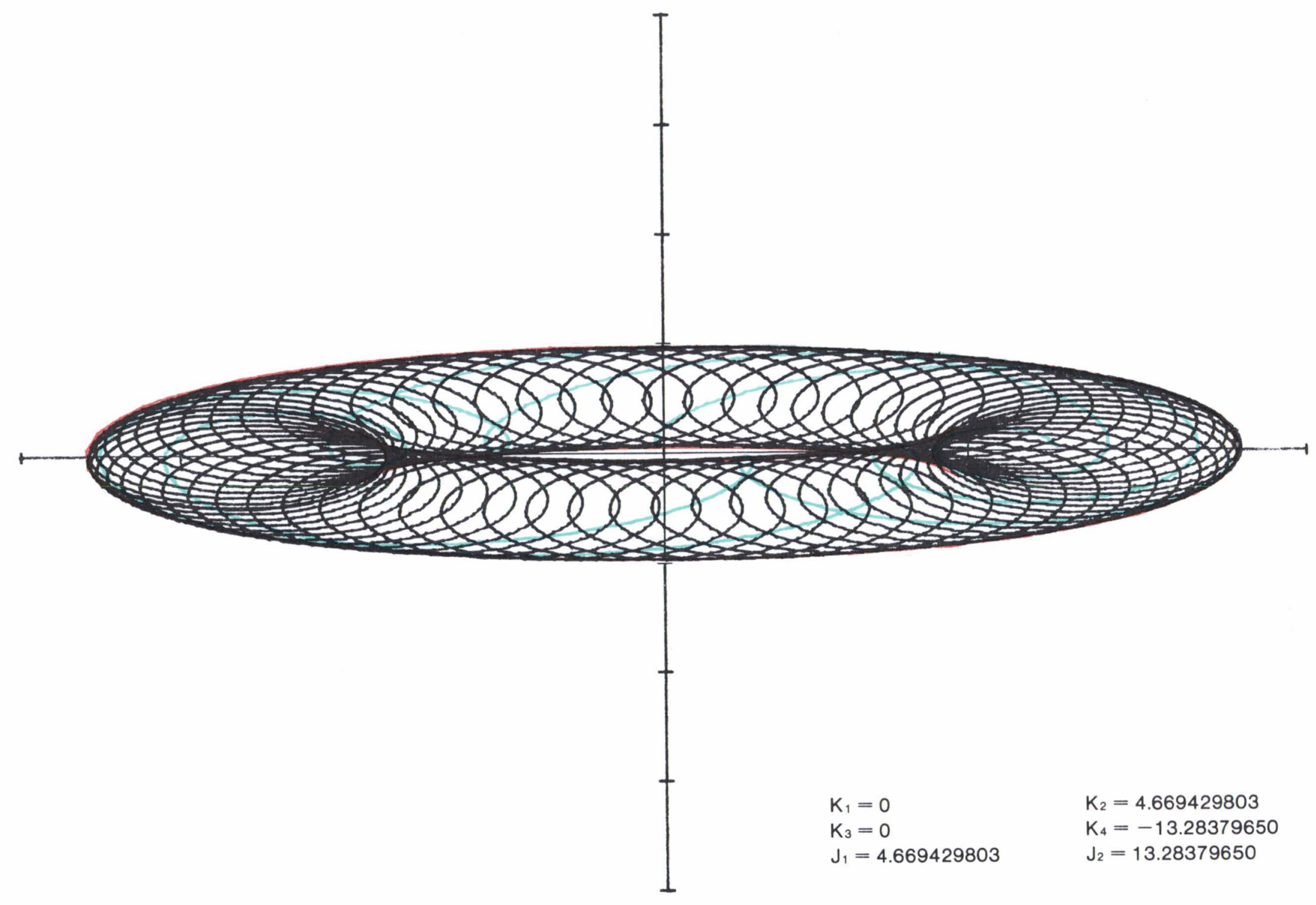

FIGURE 6.-Family of smaller ellipses $\left(\alpha=0.5, \beta=0, \gamma=270^{\circ}\right)$. 


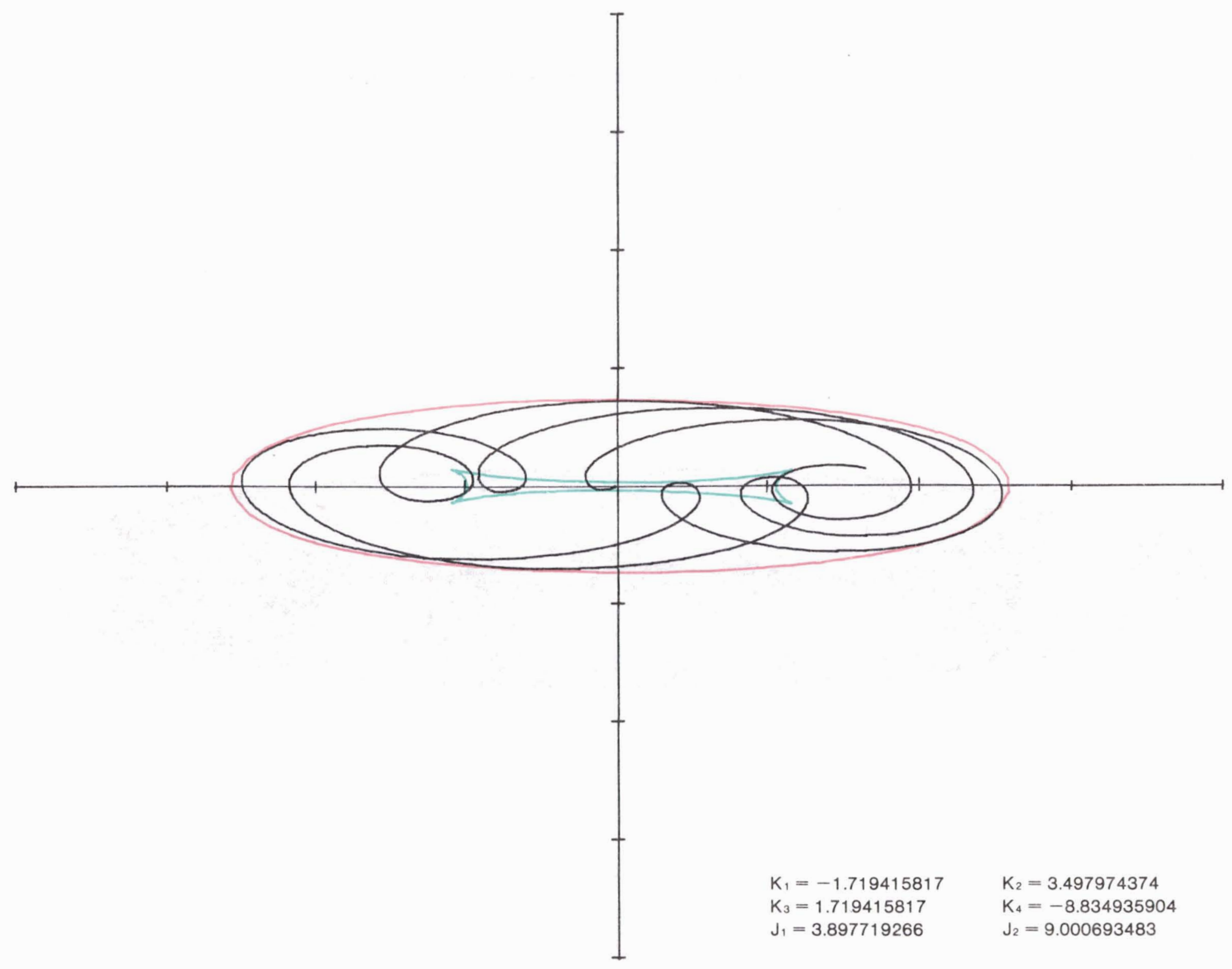

FIGURE 7.-An example for which there is no inner excluded region $\left(\alpha=0, \beta=0, \gamma=45^{\circ}\right)$. 


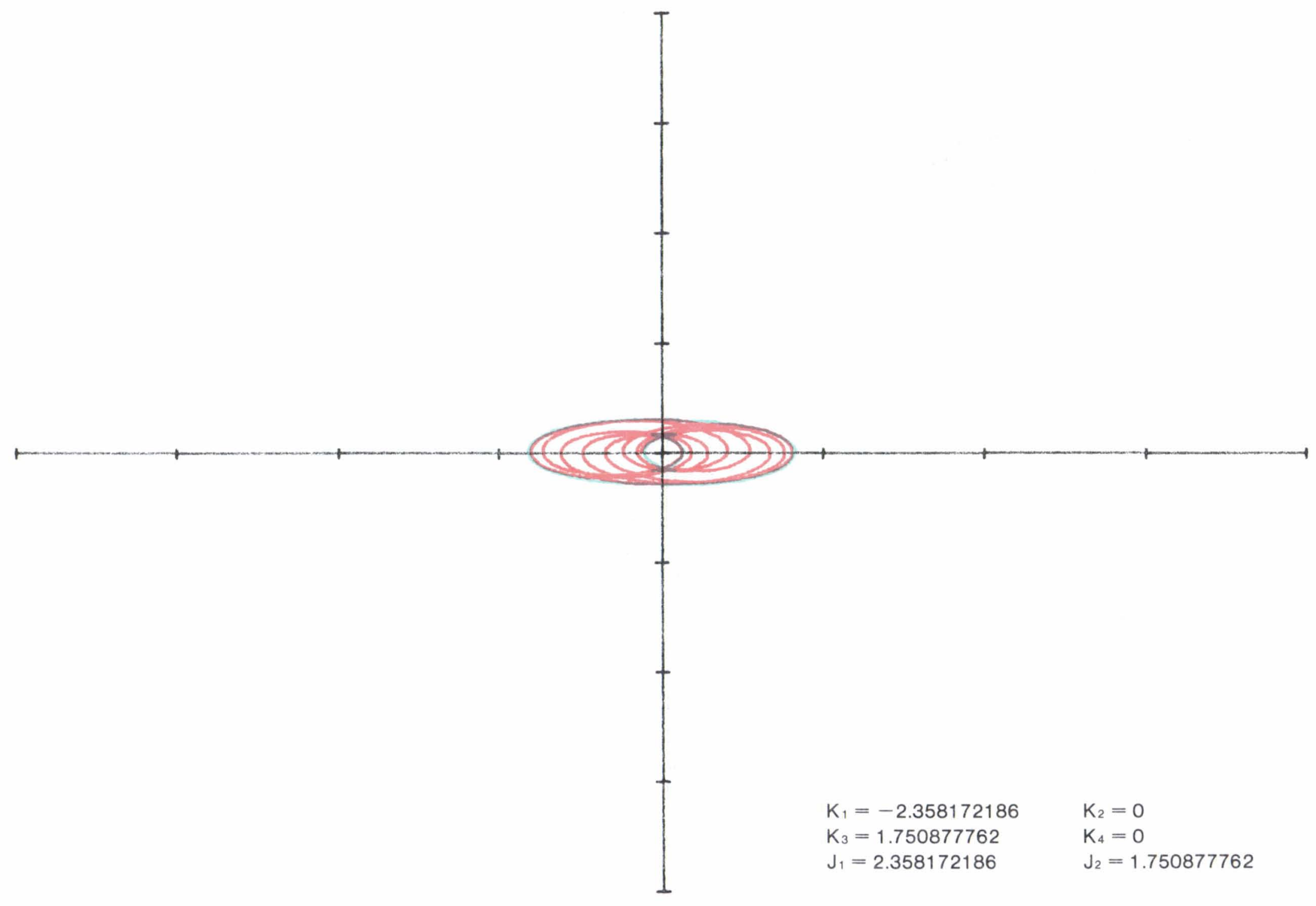

(a) $\alpha=0, \beta=-0.1, \gamma=90^{\circ}$. 


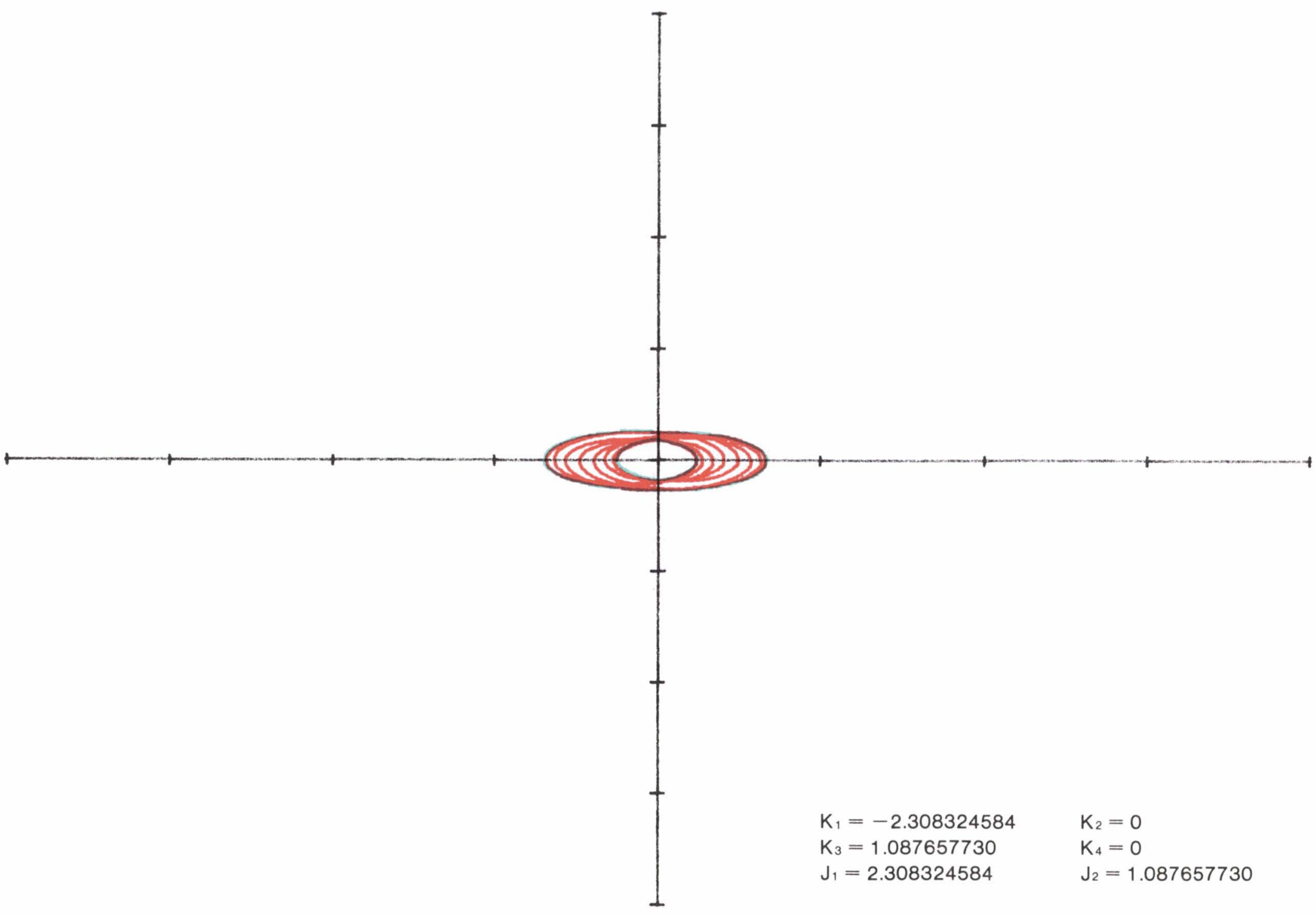

(b) $\alpha=0, \beta=-0.2, \gamma=90^{\circ}$.

FIGURE 8.-Continued. 


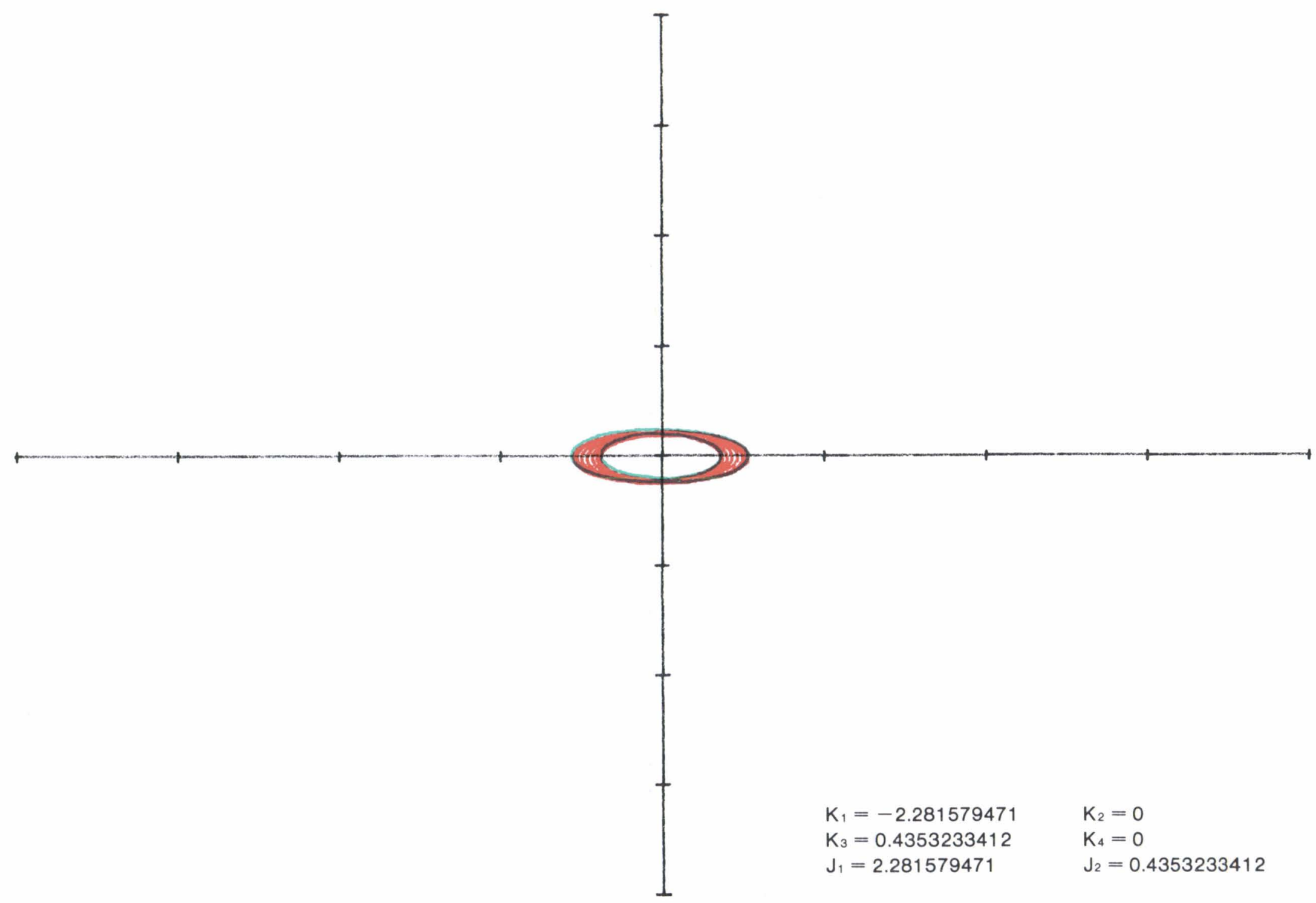

(c) $\alpha=0, \beta=-0.3, \gamma=90^{\circ}$.

FIGURE 8.-Continued. 
$\overrightarrow{+}$

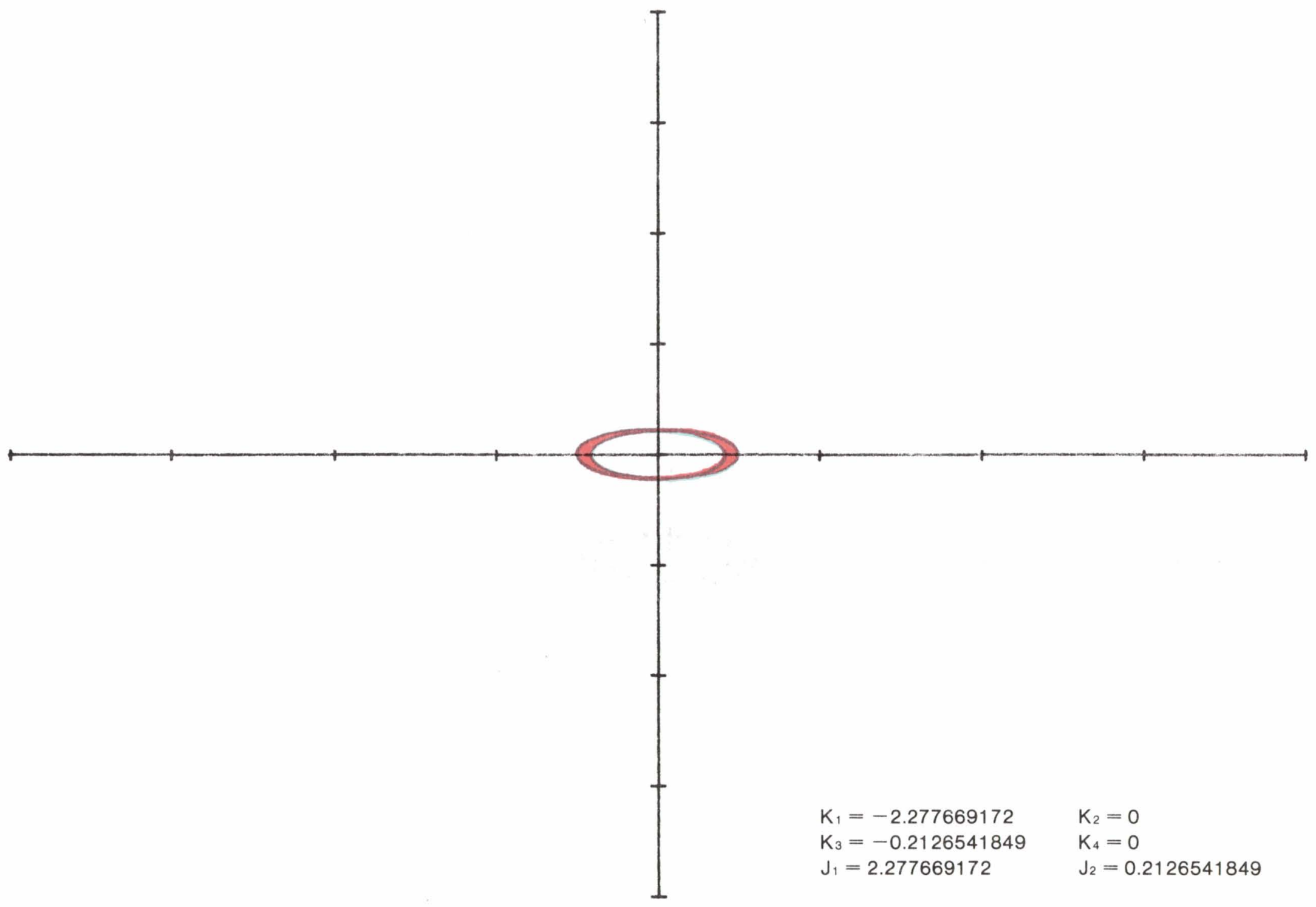

(d) $\alpha=0, \beta=-0.4, \gamma=90^{\circ}$.

FIGURE 8.-Continued. 


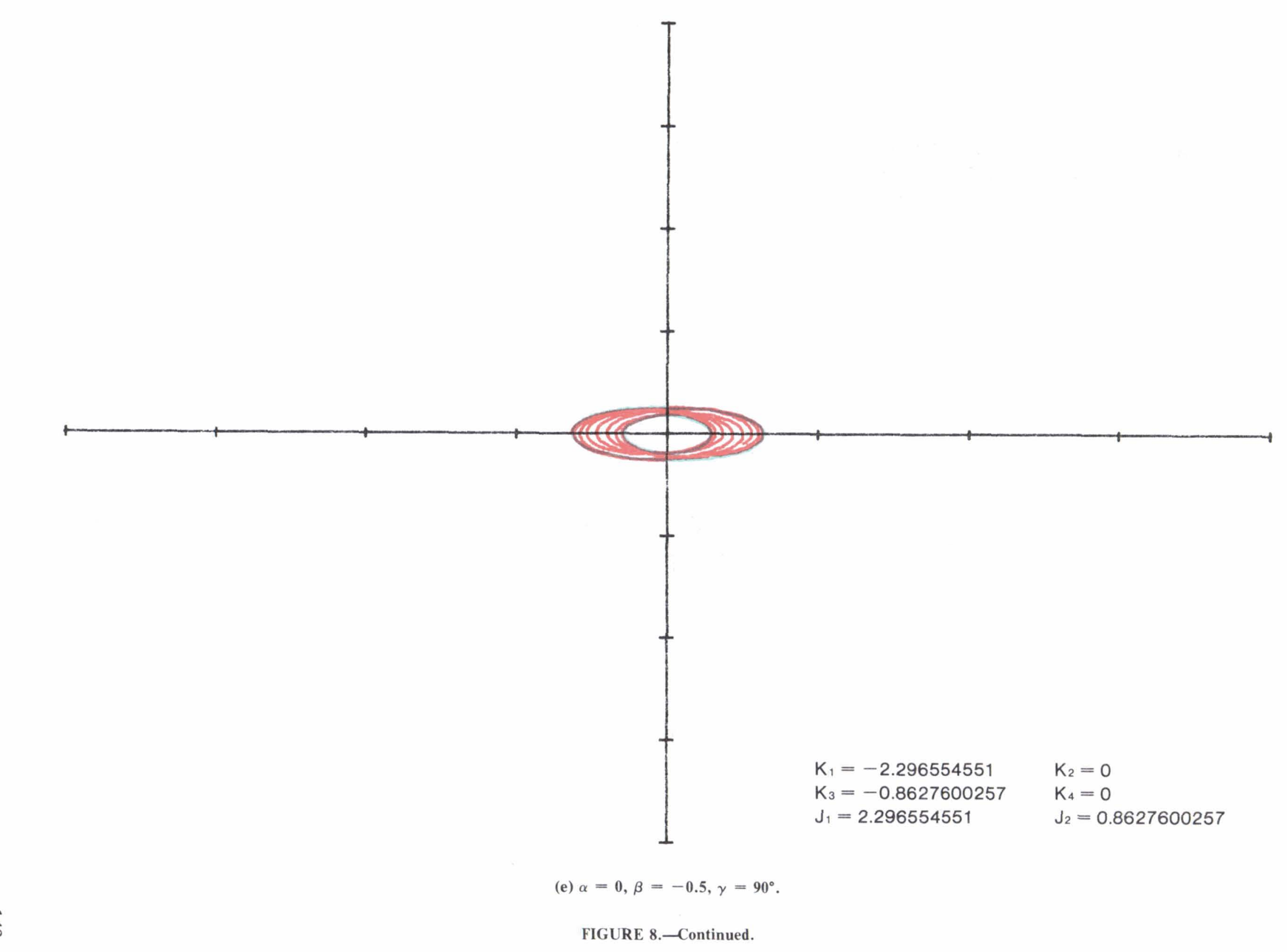




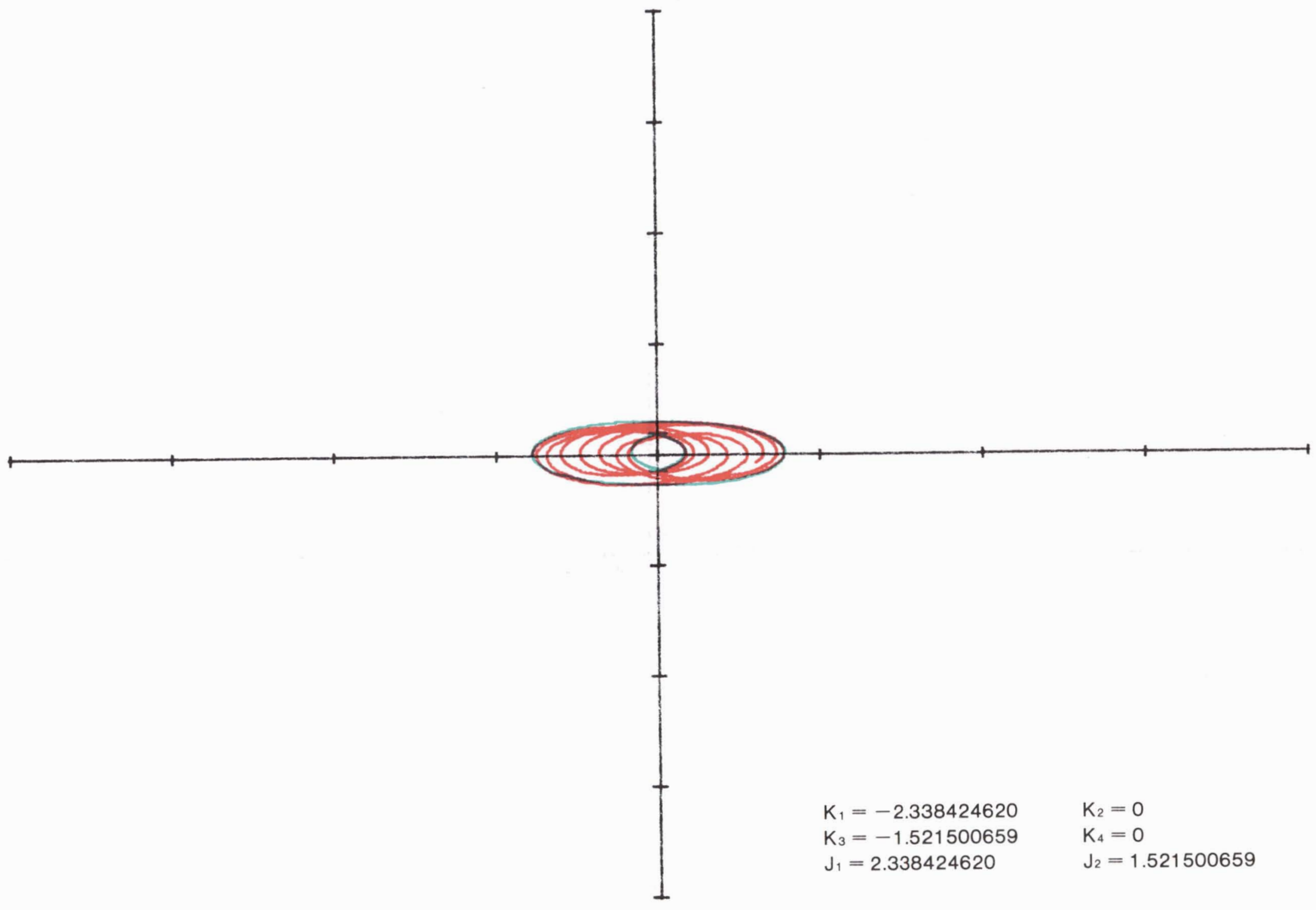

(f) $\alpha=0, \beta=-0.6, \gamma=90^{\circ}$.

FIGURE 8.-Continued. 


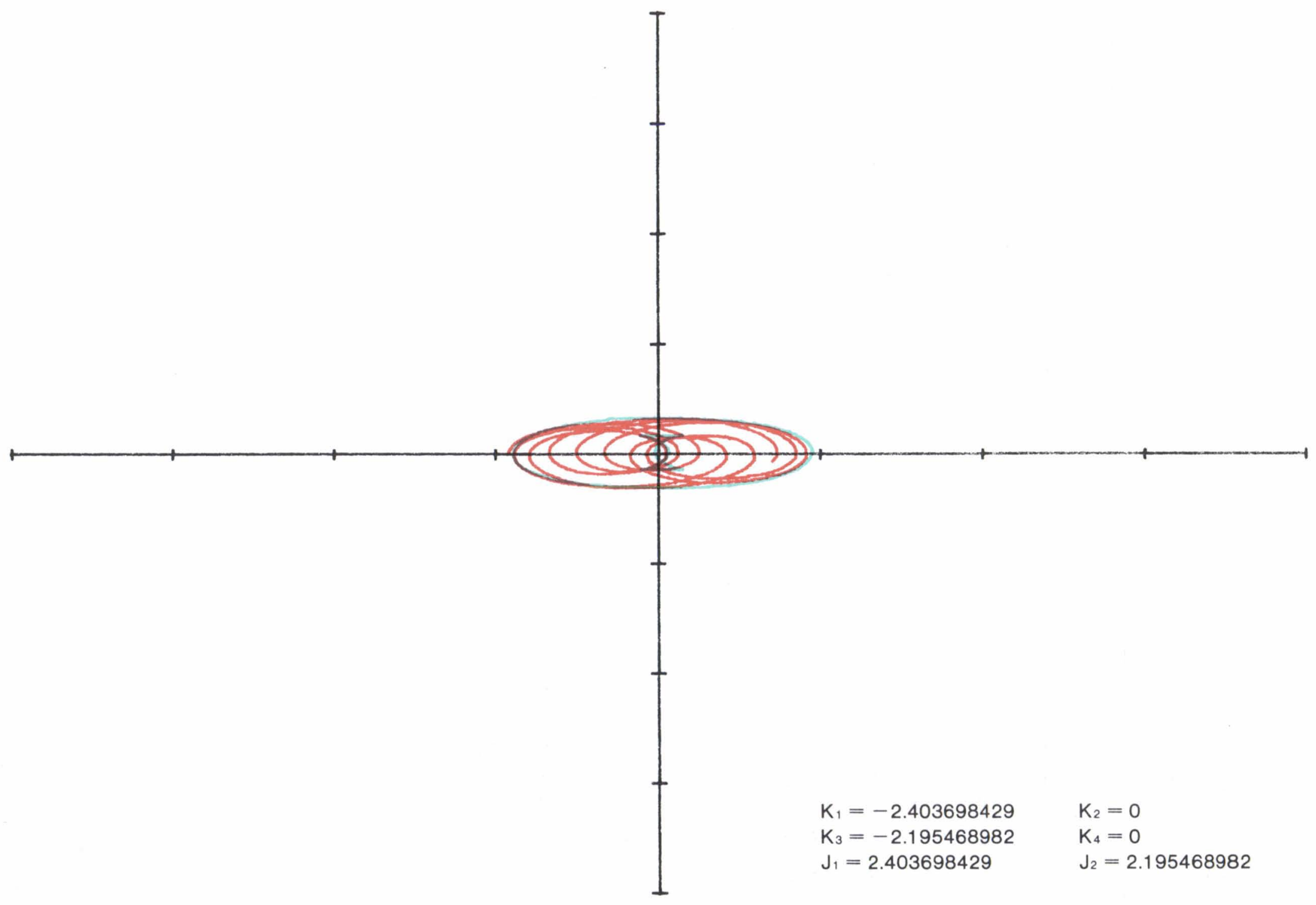

(g) $\alpha=0, \beta=-0.7, \gamma=90^{\circ}$.

FIGURE 8.-Concluded. 


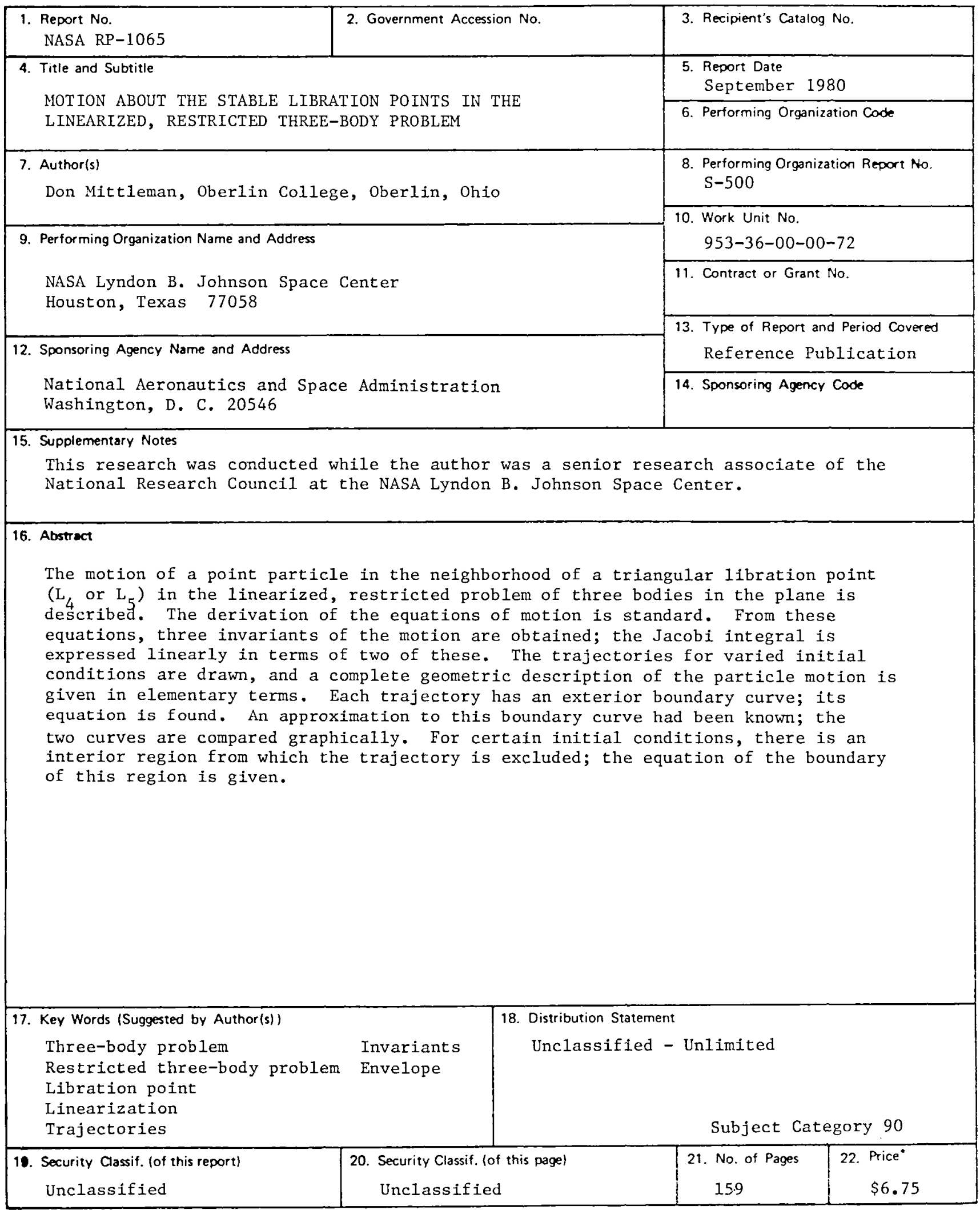



National Aeronautics and

THIRD-CLASS BULK RATE

Space Administration

Washington, D.C.

20546

Official Business

Penalty for Private Use, $\$ 300$
Postage and Fees Paid

National Aeronautics and

Space Administration

NASA-451

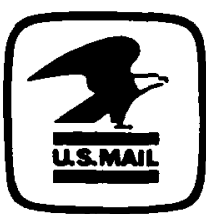

